\title{
The impact of supervised exercise therapy on intermittent claudication
}

Citation for published version (APA):

Nicolai, S. P. (2010). The impact of supervised exercise therapy on intermittent claudication. [Doctoral Thesis, Maastricht University]. Maastricht University. https://doi.org/10.26481/dis.20100319sn

Document status and date:

Published: 01/01/2010

DOI:

10.26481/dis.20100319sn

Document Version:

Publisher's PDF, also known as Version of record

\section{Please check the document version of this publication:}

- A submitted manuscript is the version of the article upon submission and before peer-review. There can be important differences between the submitted version and the official published version of record.

People interested in the research are advised to contact the author for the final version of the publication, or visit the DOI to the publisher's website.

- The final author version and the galley proof are versions of the publication after peer review.

- The final published version features the final layout of the paper including the volume, issue and page numbers.

Link to publication

\footnotetext{
General rights rights.

- You may freely distribute the URL identifying the publication in the public portal. please follow below link for the End User Agreement:

www.umlib.nl/taverne-license

Take down policy

If you believe that this document breaches copyright please contact us at:

repository@maastrichtuniversity.nl

providing details and we will investigate your claim.
}

Copyright and moral rights for the publications made accessible in the public portal are retained by the authors and/or other copyright owners and it is a condition of accessing publications that users recognise and abide by the legal requirements associated with these

- Users may download and print one copy of any publication from the public portal for the purpose of private study or research.

- You may not further distribute the material or use it for any profit-making activity or commercial gain

If the publication is distributed under the terms of Article $25 \mathrm{fa}$ of the Dutch Copyright Act, indicated by the "Taverne" license above, 


\section{The impact of supervised exercise therapy on intermittent claudication}




\section{PROEFSCHRIFT}

Ter verkriiging van de graad van doctor aan de Universiteit Maastrich op gezag van de Rector Magnificus, Prof. Mr. G.P.M.F. Mols

volgens het besluit van het College van Decanen

in het openbaar te verdedigen

op vrijdag 19 maart 2010 om 14.00 uur

door

Saskia Patricia Anna Nicolaï

\section{The impact} of supervised exercise therapy on intermittent claudication 
Promotor

Prof. dr. M.H. Prins

\section{Copromotores}

Dr. J.A.W. Teijink (Catharina-ziekenhuis, Eindhoven)

Dr. E.V. Rouwet (Erasmus Medisch Centrum, Rotterdam)

\section{Beoordelingscommissie}

Prof. dr. M.J.H.M. Jacobs (voorzitter)

Prof. dr. ir. P.A. van den Brandt

Prof. dr. H.R. Büller (Academisch Medisch Centrum, Amsterdam)

Dr. A.G.M. Hoofwijik (Orbis Medisch Centrum, Sittard)

Prof. dr. J.F.M. Metsemakers 


\section{Vormgeving \& Layout:}

infour marketing \& communicatie

\section{Drukkerij:}

Drukkerij Pietermans

\section{ISBN/EAN: 978-90-9025047-2}

\section{The publication of this thesis was financially sponsord by}

The EXITPAD study, a part of the research described in this thesis was supported by a grant (no. 945-06-252) from ZonMw.

Financial support by the Netherlands Heart Foundation for the publication of this thesis is gratefully acknowledged.

Additional financial support was generously provided by; Atrium medisch centrum Parkstad, Astra Zeneca B.V., Fastguide B.V., Sigma medical B.V., VSM geneesmiddelen B.V Bauerfeind Benelux B.V., Bard Benelux N.V., W.L. Gore \& Associates B.V., Eli Lilly Nederland B.V., Sanofi-Aventis Netherlands B.V, Krijnen Medical Innovations, and COOK Medical. 


\section{Content of the thesis}

Introduction and outline of the thesis

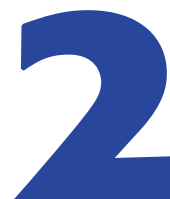

Pocket Doppler and vascular laboratory equipment

yield comparable results for ankle brachial

index measurement

BMC Cardiovasc Disord. 2008;8:26

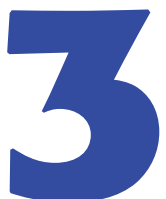

Ankle brachial index measurement in primary care

are we doing it right?

Br J Gen Prac. 2009;59:422-7
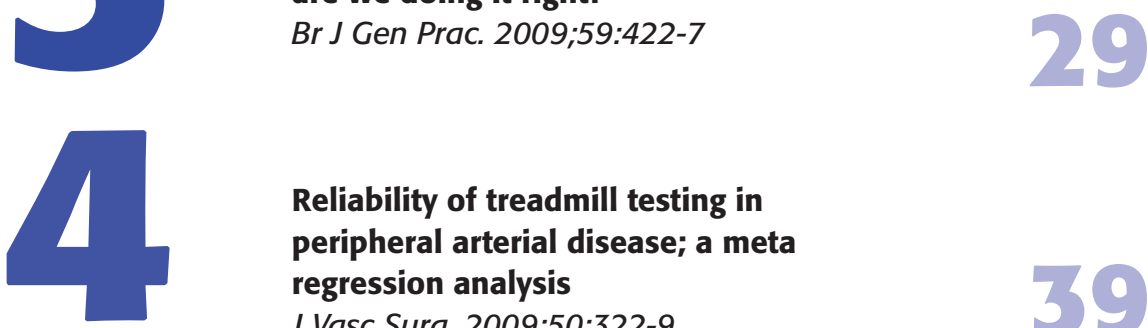

Reliability of treadmill testing in

peripheral arterial disease; a meta

regression analysis

J Vasc Surg. 2009;50:322-9

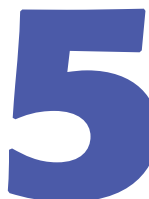

Extending the range of treadmill testing

for patients with peripheral arterial disease

Med Sci Sports Exerc. 2009;[epub ahead of print]

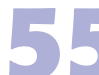

Validation of the Dutch version

of the Walking Impairment Questionnaire

Eur J Vasc Endovasc Surg. 2009;37:56-61

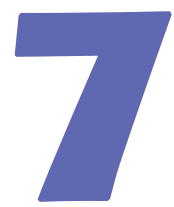

The walking impairment questionnaire: An effective tool to assess the effect of treatment in patients with intermittent claudication

J Vasc Surg. 2009;50:89-94

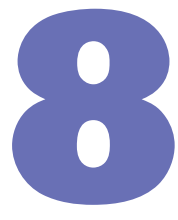

Supervised exercise therapy for intermittent claudication in daily practice: one-year results

of a community-based approach

J Vasc Surg. 2009;37:56-61

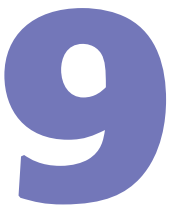

Multicenter randomized clinical trial of supervised exercise therapy with or without feedback versus walking advice for intermittent

claudication

Submitted

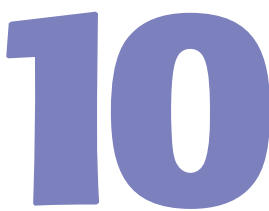

Optimization of supervised exercise

programs for patients with intermittent claudication

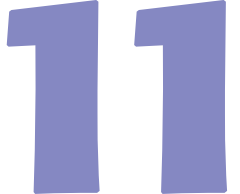

General discussion

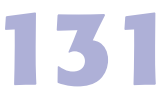

Appendix

Summary

Samenvatting

158

Dankwoord

165

Curriculum Vitae 
CHAPTER 1

Introduction

and outline of the thesis 


\section{Peripheral arterial disease}

Peripheral arterial disease (PAD) is a manifestation of systemic atherosclerosis leading to narrowing of the arteries in the legs. The most common symptom of PAD is intermittent claudication, which classically manifests as cramping pain in calf, thigh, and/or buttock muscles occurring during exercise and subsiding rapidly with rest. In more severe cases PAD leads to acute or critical limb ischemia. In addition to symptomatic PAD, 20-50\% of people with PAD are asymptomatic. ${ }^{13}$ Importantly, about 15\% of people aged 50 years or older have asymptomatic PAD. Overall, the reported combined prevalence of symptomatic and asymptomatic PAD in the Netherlands is $19.1 \%$ in persons aged 55 years and over. ${ }^{4}$

\section{Ankle Brachial Index}

Usually PAD can not accurately be diagnosed based on the vascular history and physical examination alone. ${ }^{5}$ Although the majority of patients with PAD have a limited exercise performance, only a minority present with typical claudication symptoms.

Furthermore, absence of pedal pulses on physical examination tends to over-diagnose PAD.5 The ankle brachial index (ABI) is the gold standard to evaluate the presence of $\mathrm{PAD}$, and thus the presence of atherosclerosis in arteries of the leg as a reflection of generalized atherosclerosis. ${ }^{6}$ The $\mathrm{ABI}$ is determined by measuring the systolic blood pressure in both arms and the systolic pressures in the dorsal pedal and posterior tibia arteries of both legs. The ABI is the quotient of the highest systolic pressure at each ankle divided by the highest of the two brachial pressures.

Normally, the systolic blood pressure at the ankle exceeds the brachial pressure by 12 to $24 \mathrm{mmHg}$ due to reflection of blood pressure waves from the high resistance peripheral arteriolar bed in the lower extremity. ${ }^{8} \mathrm{As}$ a result, an $\mathrm{ABI}$ between 1.0 and 1.3-1.4 is considered normal. ${ }^{9} \mathrm{~A}$ hemodynamically significant stenosis due to atherosclerosis in the lower extremity arteries induces a decrease in the systolic ankle pressure, thus yielding an $\mathrm{ABI}$ of 0.9 or less. $\mathrm{An} \mathrm{ABI}<0.9$ is a reliable determinant of PAD, with a sensitivity of $79-95 \%$ and a specificity of $96-100 \% .{ }^{9,} 10$ In the general population, an $\mathrm{ABI}<0.9$ is associated with a 10-year cardiovascular mortality rate of approximately 15\%.6 The lower the ABI, the greater the cardiovascular morbidity and mortality. Five-year mortality is $30 \%$ in patients with an $\mathrm{ABI}<0.7$, and even $50 \%$ in those with an $\mathrm{ABI}<0.4 .^{1{ }^{1-13}}$ The $\mathrm{ABI}$ may be falsely elevated (>1.3-1.4) in case of noncompressible arteries at the ankle due to arterial calcification, as may occur in patients with diabetes or end-stage renal disease, and in the elderly.

Occasionally, resting $A B I$ may be normal in patients with $P A D$, for example in patients with aortoiliac stenoses and a well-collateralized arterial system in combination with lowered resistance in the peripheral arteriolar runoff bed. However, exercise induces further decrease in arteriolar resistance to increase blood flow to the exercising muscles. The increased blood flow through stenotic flow-limiting arteries causes a drop in the ankle pressure, which gradually recovers after exercise cessation., 14 These changes are detected clinically by a fall in the ABI as determined within several minutes of exercise cessation. Thus, exercise testing increases the sensitivity of the $A B$ I method for evaluating patients with typical symptoms of claudication in whom the resting $\mathrm{ABI}$ is normal. A decrease in $\mathrm{ABI}$ of $0.15(15 \%)$ after exercise is associated with PAD.7, 15 The ABI can be determined by a simple handheld pocket Doppler and a

sphygmomanometer cuff, or by more advanced automated equipment in a vascula laboratory. Because the $\mathrm{ABI}$ is a relatively easy and inexpensive measurement, it is increasingly used in primary care to establish the diagnosis of (a)symptomatic PAD and to assess cardiovascular risk.

\section{Evaluation of functional capacity in patients with intermittent claudication}

Treadmill testing

Patients with intermittent claudication have a limited walking ability. Traditionally, walking ability is expressed as the maximal walking distance in meters as assessed by treadmill testing. Several treadmill protocols with different speeds and inclines are used to quantify walking distance, but each falls into one of two types: constant or graded protocols. In both types of protocols patients exercise until they are unable to continue (absolute claudication distance). The initial claudication distance is the moment claudication pain appears. Furthermore, the functional claudication distance $(F C D)$, which is the moment the patient prefers to stop because of claudication pain, is recently added by our research group as a reliable and valid outcome measurement. ${ }^{16}$ The conventional tests use a constant exercise protocol; a constant specific speed (2 to $4 \mathrm{~km}$ per hour) and treadmill grade (0\% to 12\%) is chosen. In the 1970s, Bruce et al. developed a graded test with a low initial workload which increased during the test, to perform functional assessments of patients with cardiac disease. ${ }^{17}$ Later, the graded testing concept was extended to patients with PAD. Nowadays, the two most commonly applied graded protocols have a constant speed of $3.2 \mathrm{~km} / \mathrm{h}$, increasing the incline every 2 minutes with $2 \%,{ }^{18}$ or every 3 minutes with $3.5 \%{ }^{19}$

Unfortunately, the great variety of treadmill test protocols hampers the comparison of studies on the effects of therapy for intermittent claudication. Furthermore, treadmill testing is impractical in daily practice, since it is time-consuming, requires adequate equipment and personnel, is not directly available to all physicians, and is not suitable for all claudication patients due to cardiopulmonary or musculoskeletal co morbidities. Alternative tests to quantify walking ability, including the six-minute walking test, a shuttle walking test, a Peripheral Arterial Disease Holter Control (PADHOC) device, or a global positioning system (GPS), are not widely used.

Quality of life assessment

In addition to walking limitation, assessment of quality of life is important in the evaluation of patients with intermittent claudication. Current guidelines recommend to evaluate PAD by means of a validated, disease-specific health status questionnaire or on physical function domains from validated generic health status questionnaires. ${ }^{7}$ Generic questionnaires, such as the Short Form 36 (SF-36) (or RAND-36 with a slightly different scoring algorithm) or the European Quality of Life 5D (EuroQol-5D) can be used for quality of life evaluation for a variety of diseases as well as for costeffectiveness analysis. However, generic questionnaires, which do not focus on specific complaints of PAD, are considered to be less sensitive to detect therapy effect. ${ }^{20}$

Disease-specific questionnaires have been developed for the assessment of patient with $\mathrm{PAD}$, including the Walking Impairment Questionnaire (WIQ), ${ }^{21}$ the Kings College Vascular Quality of Life Questionnaire (VASCUOOL) ${ }^{22}$ and the claudication scale (CLAUS). ${ }^{23}$ 


\section{Management of patients with intermittent claudication}

Secondary prevention of cardiovascular disease

Treatment of patients with PAD consists of both secondary prevention measures for atherosclerosis and symptomatic relief. PAD is one of the major manifestations of systemic atherosclerosis, along with coronary artery disease and cerebrovascular disease. Patients with PAD have a 5.5 fold increased risk of a cardiovascular death and a 2.5 fold higher risk of coronary artery disease or stroke.3, 24,25 Identification and modification of the well known risk factors for atherosclerosis, including advanced age, smoking, diabetes mellitus, dyslipidemia, hypertension, and obesity, are highly important to reduce the risk of myocardial infarction or stroke in this patient population Cardiovascular risk management consists of antiplatelet therapy, statin therapy, treatment of hypertension, tight glycemic control, and life style interventions for weight reduction, tobacco smoking cessation, and stimulation of physical activity.

\section{Supervised exercise therapy}

According to current national and international guidelines, exercise therapy is the first-line symptomatic treatment for patients with intermittent claudication.7, 9,15 Based on the idea of Thoma in 1884 that the growth of collateral blood vessels was directly proportional to blood flow ${ }_{1}^{26}$ walking was suggested as a therapy for patients with PAD. The concept of exercise therapy for the treatment of intermittent claudication has regained attention in the 1950s. ${ }^{27,28}$ In 1966, Larsen and Lassen conducted the first randomised clinical trial on structured exercise therapy. ${ }^{29}$ Nowadays exercise therapy is advised to consist of intermittent treadmill exercise at a speed and incline that will induce claudication within 3 to 5 minutes. After reaching near-maximal leg pain, a resting period follows in which claudication pain abates. Then, treadmill walking is restarted. A program of at least 6 months with 3 or more training sessions per week of at least 30 minutes is suggested to benefit most. ${ }^{30}$ Although exercise has shown efficacious to improve exercise performance in patients with $P A D$, the underlying mechanisms are still poorly understood. Current evidence does not support an increase in blood flow as the primary factor for improvement in walking distance, since an increase in walking distance after supervised exercise therapy is not necessarily associated with an increase in $\left.A B\right|^{30,31}$ Theoretically, growth factors such as Vascular Endothelial Growth Factor which are released during tissue hypoxia may promote angiogenesis and collateral blood vessel formation in leg muscles in response to exercise therapy. ${ }^{32}$ Although this concept seems to be attractive, it remains to be proven. ${ }^{33}$ Other potential mechanisms include changes in microcirculation and endothelial function, ${ }^{34}$ improved hemorrheology after exercise, ${ }^{35}$ changes in skeletal muscle metabolism, ${ }^{36}$ or physiological adaptations such as a reduced heart rate during exercise and enhanced peak exercise oxygen consumption. ${ }^{37,38}$ Alternatively, increased walking efficacy and alteration in gait may contribute to the regained walking distance. ${ }^{39}$

In several small clinical trials it has been demonstrated that supervised exercise therapy is significantly more effective than non-supervised exercise therapy in improving walking distance in patients with intermittent claudication. ${ }^{40}$ Studies assessing the effect of supervised exercise therapy on quality of life are scarce. Published data suggest that supervised exercise therapy has no or only a modest benefit on quality of life. ${ }^{40-42}$

\section{Organisation of supervised exercise therapy}

The TransAtlantic Inter-Society Consensus (TASC-II) and the American Heart Association practice guidelines on the management of patients with PAD advice that supervised exercise should be made available as part of the initial treatment for all patients suffering from intermittent claudication., 73 Infrastructure for and content of supervised exercise programs vary widely between studies with various advantages and disadvantages for professionals performing and patients following the program.

\section{Aim and outline of the thesis}

The aim of this thesis is to refine the diagnostic workup and conservative treatment of patients with PAD. To this end, a number of studies were conducted focusing on diagnosis of PAD, assessment of disease severity and effectiveness of therapy, and supervised exercise therapy for intermittent claudication.

Since the diagnosis of PAD is primarily established by a resting $\mathrm{ABI}<0.9$ and/or a decrease of $>0.15$ after exercise, accurate determination of the $A B I$ is crucial in the diagnostic workup of patients with PAD. In Chapter 2, ABI measurements with a pocket Doppler are compared with $A B I$ measurements with the use of vascular laboratory equipment. In Chapter $\mathbf{3}$, the implementation of $\mathrm{ABI}$ measurements in primary care practices using pocket Doppler devices is investigated.

Treadmill testing is the gold standard for the assessment of walking ability. In Chapter 4, a meta-analysis is presented to determine which treadmill protocol is most reliable to assess the majority of patients with PAD. Since not all patients are able to walk on a 'standard' protocol, two new protocols, one with a lower workload and one with a higher workload, are developed in Chapter $\mathbf{5}$.

The importance of functional walking ability instead of walking distance assessed by treadmill testing is increasingly recognized. The Walking Impairment Questionnaire (WIQ) is a questionnaire evaluating functional walking ability on three domains

Chapter 6 presents the validation of the Dutch version of the WIQ using the European metric system. Furthermore, Chapter $\mathbf{7}$ describes the validity of the WIQ to assess objective improvement in functional walking ability after supervised exercise therapy. Supervised exercise therapy is recommended as the initial symptomatic treatment for the majority of patients with intermittent claudication. The Atrium medical center organized a network of regional physiotherapists in the Parkstad area to provide structured supervised exercise therapy in close proximity to the patient's home (Figure 1).4 All participating physiotherapists were trained in supervised exercise programs according to the Dutch guideline 'Intermittent Claudication'.45 In Chapter 8, a cohort study is presented with the results of supervised exercise therapy provided by regional physiotherapists. To investigate the effectiveness of supervised exercise therapy in patients with intermittent claudication with or without feedback provided by regional physiotherapists, compared with non-supervised therapy a multicenter randomized trial is presented in Chapter 9. Effectiveness of supervised exercise therapy is expressed as the change in walking distance, scores on the WIQ, and quality of life. The central aim of this Exercise Therapy in Peripheral Arterial Disease (EXITPAD) study is to improve the adherence to exercise therapy. Therefore, outcome measurements of patients with a 'go home and walk' advice are compared with patients following supervised exercise therapy and with patients following supervised exercise therapy 
with additional supply of an accelerometer displaying the daily amount of physical activity. In this study the hypothesis is tested that daily feedback by an accelerometer increases physical activity, resulting in improved walking ability. Chapter 10

investigates the optimal training intensity of supervised exercise therapy to achieve maximal improvement in walking distance.

The thesis concludes with a general discussion of the results of these studies and prospects for the management of patients with PAD in Chapter 11.

Figure 1: The network for supervised exercise therapy of the Atrium medical center Parkstad, Heerlen.

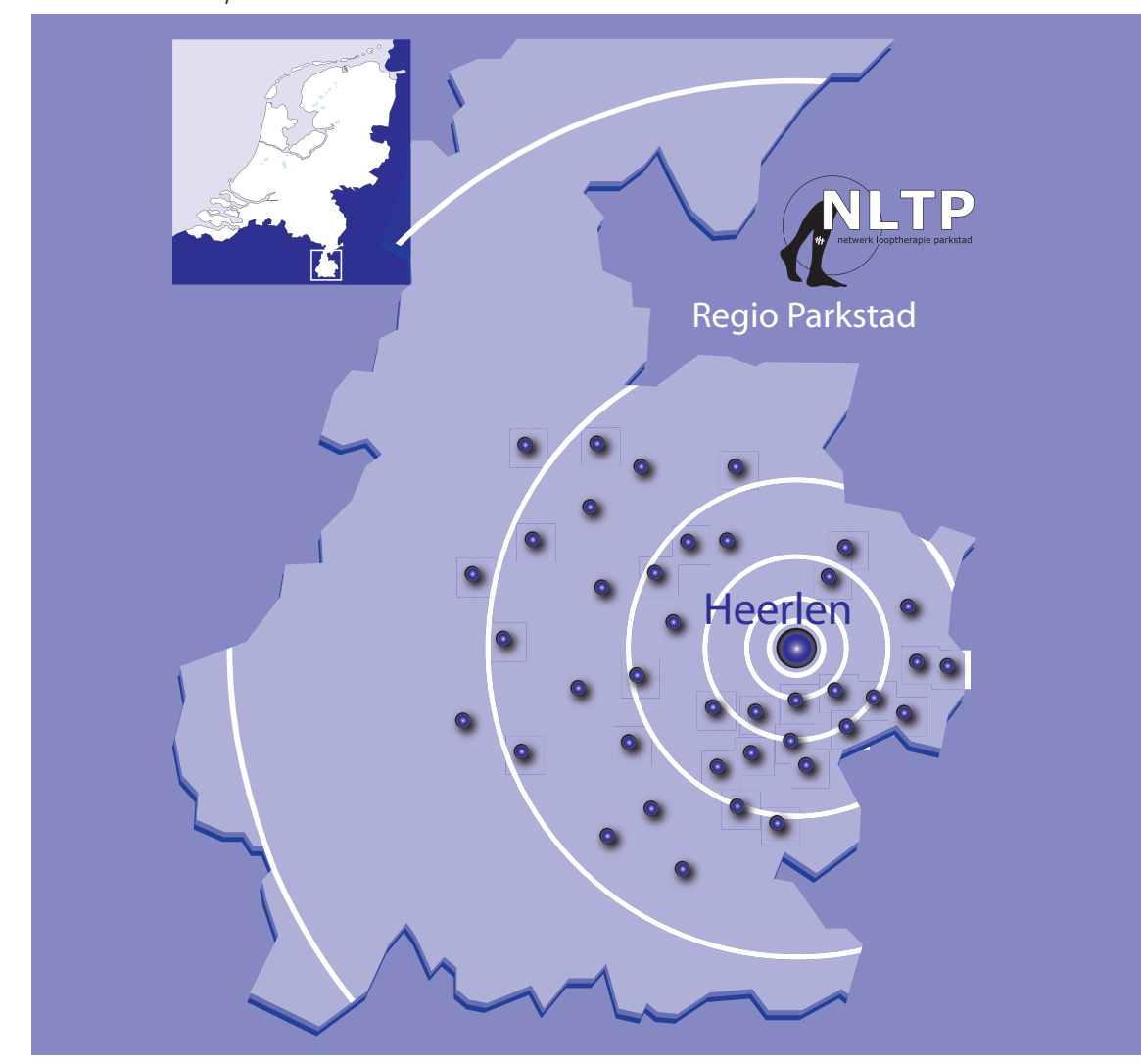

\section{References}

01. McDermott MM, Greenland P, Liu K, Guralnik JM, et al. Leg symptoms in peripheral arterial disease: associated clinical characteristics and functiona impairment. JAMA. 2001;286:1599-1606.

02. McDermott MM, Fried L, Simonsick E, Ling S, Guralnik JM. Asymptomatic peripheral arterial disease is independently associated with impaired lower extremity functioning: the women's health and aging study. Circulation. 2000;101:1007-1012.

03. Hirsch AT, Criqui MH, Treat-Jacobson D, et al. Peripheral arterial disease detection, awareness, and treatment in primary care. JAMA 2001;286:1317-1324.

04. Meijer WT, Hoes AW, Rutgers D, Bots ML Hofman A, Grobbee DE. Peripheral arterial disease in the elderly: The Rotterdam Study. Arterioscler Thromb Vasc Biol. 1998;18:185-192.

05. Khan NA, Rahim SA, Anand SS, Simel DL, Panju A. Does the clinical examination predict lower extremity peripheral arterial disease? JAMA 2006:295:536-546.

06. Fowkes FG, Murray GD, Butcher I, Heald CL, Lee RJ, Chambless LE, Folsom AR, Hirsch AT, Dramaix M, deBacker $\mathrm{G}$, et al. Ankle brachial index combined with Framingham Risk Score to predict cardiovascular events and mortality: a meta-analysis. JAMA. 2008;300:197-208.

07. Norgren L, Hiatt WR, Dormandy JA, Nehler MR, Harris KA, Fowkes FG. Inter-Society Consensus for the Management of Peripheral Arterial Disease (TASC II). J Vasc Surg. 2007;45 Suppl S:S5-67.

08. Carter SA. Response of ankle systolic pressure to leg exercise in mild or questionable arterial disease. New Engl J Med. 1972;287:578-582.

09. Bartelink ML, Boutens EJ, Hooi JD, Kaiser V Boomsma $\perp$ NHG-standaard perifeer arteriee vartlijen Huisarts Wet 2003:46:848-858
10. Fowkes FC, Housley E, Macintyre CC, Prescott RJ, Ruckley CV. Variability of ankle and brachial systolic pressures in the measurement of atherosclerotic peripheral arterial disease. J Epidemiol Community Heath. 1988;42:128-133.

11. Vogt MT, McKenna M, Anderson SJ, Wolfson SK, Kuller LH. The relationship between ankle-arm index and mortality in older men and women. Am Geriatr Soc. 1993:41:523-530.

12. Sikkink CJ, van Asten WN, van 't Hof MA, van Langen $\mathrm{H}$, van der Vliet JA. Decreased ankle/ brachial indices in relation to morbidity and mortality in patients with peripheral arterial disease. Vasc Med. 1997;2:169-173.

13. McKenna M, Wolfson S, Kuller L. The ratio of ankle and arm arterial pressure as an independent predictor of mortality. Atherosclerosis. 1991:87:119-128.

14. Ouriel K, MCDonnell AE, Metz CE, Zarins CK. Critical evaluation of stress testing in the diagnosis of peripheral vascular disease. Surgery. 1982;91:686-693.

15. Vahl AC, Reekers JA. The guideline 'Diagnosis and treatment of peripheral artery disease of the lower extremities' of The Netherlands Surgical Society]. Ned Tijdschr Geneeskd. 2005;149:1670-1674.

16. Kruidenier LM, Nicolaï SP, Willigendael EM, de Bie RA, Prins MH, Teiijink JA. Functional claudication distance: a reliable and valid measurement to assess functional limitation in patients with intermitten claudication. BMC cardiovascular disorders. 2009;9:9.

17. Bruce RA, Kusumi F, Hosmer D. Maximal oxygen intake and nomographic assessment of functiona aerobic impairment in cardiovascular disease. Am Heart J. 1973;85:546-562.

18. Cardner AW, Skinner JS, Cantwell BW, Smith LK Progressive vs single-stage treadmill tests for evaluation of claudication. Med Sci Sports Exerc 1991:23:402-408 
19. Hiatt WR, Nawaz D, Regensteiner JG, Hossack $\mathrm{KF}$. The evaluation of exercise performance in patients with peripheral vascular disease. J Cardiopulmonary Rehabil. 1988;12:525-532.

20. Mehta T, Venkata Subramaniam A, Chetter McCollum P. Assessing the validity and responsiveness of disease-specific quality of life instruments in intermittent claudication. Eur J Vasc Endovasc Surg. 2006;31:46-52.

21. McDermott MM, Liu K, Guralnik JM, Martin GJ, Criqui MH, Greenland P. Measurement of walking endurance and walking velocity with questionnaire: validation of the walking impairment questionnaire in men and women with peripheral arterial disease.J Vasc Surg. 1998;28:1072-1081.

22. Morgan MB, Crayford T, Murrin B, Frase SC. Developing the Vascular Quality of Life Questionnaire: a new disease-specific quality of life measure for use in lower limb ischemia. J Vasc Surg. 2001;33:679-687.

23. Finger T, Kirchberger I, Dietze $S$, van Laak $H$, Comte $S$. Assessing the quality of life of patients with intermittent claudication; psychometric properties of the claudication scale (CLAU-S). Qual Life Res 1995;4:427.

24. Doobay AV, Anand SS. Sensitivity and specificity of the ankle-brachial index to predict future cardiovascular outcomes: a systematic review. Arterioscler Thromb Vasc Biol. 2005;25:1463-1469.

25. Bhatt DL, Steg PG, Ohman EM, et al. International prevalence, recognition, and treatment of cardiovascular risk factors in outpatients with atherothrombosis. IAMA. 2006:295:180-189.

26. Thoma R. Über die Abhangigkeit der Bindegewebsneubildung in der Arterien intima von dermechanischen Bedingungen de Blutumlaufes. Virchows Arch. 1884(85):294.
27. Wisham LH, Abramson AS, Ebel A. Value of exercise therapy in peripheral arterial disease. JAMA. 1953(153):10.

28. Foley WT. Treatment of gangrene of the feet and egs by walking. Circulation. 1957(15):689-700.

29. Larsen OA, Lassen NA. Effect of daily muscular exercise in patients with intermittent claudication Lancet. 1966;2:1093-1096.

30. Gardner AW, Poehlman ET. Exercise rehabilitation programs for the treatment of claudication pain. A meta-analysis. JAMA. 1995;274:975-980

31. Watson L, Ellis B, Leng GC. Exercise for intermittent claudication. Cochrane database of systematic reviews (Online). 2008(4):CD000990.

32. Gustafsson T, Bodin K, Sylven C, Gordon A, TyniLenne $R$, Jansson E. Increased expression of VEC following exercise training in patients with hear failure. Eur J Clin invest. 2001;31:362-366.

33. Stewart KJ, Hiatt WR, Regensteiner JG, Hirsch AT. Exercise training for claudication. New Engl J Med. 2002;347:1941-1951.

34. Brendle DC, Joseph $\sqcup$, Corretti MC, Gardner AW, Katzel L. Effects of exercise rehabilitation on endothelial reactivity in older patients with peripheral arterial disease. Am J Cardiol. 2001:87·324-329.

35. Capecchi PL, Pasini FL, Cati G, et al. Experimenta model of short-time exercise-induced preconditioning in POAD patients. Angiology. 1997;48:469-480.

36. Lundgren F, Dahllof AG, Schersten T, BylundFellenius AC. Muscle enzyme adaptation in patients with peripheral arterial insufficiency: spontaneous adaptation, effect of different treatments and consequences on walking performance Clin Sci. 1989;77:485-493.
37. Hiatt WR, Regensteiner JG, Hargarten ME, Wolfel $E E$, Brass EP. Benefit of exercise conditioning for patients with peripheral arterial disease. Circulation. 1990;81:602-609.

38. Hiatt WR, Wolfel EE, Meier RH, Regensteiner JG. Superiority of treadmill walking exercise versus strength training for patients with peripheral arteria disease. Implications for the mechanism of the training response. Circulation. 1994;90:1866-1874

39. Womack CJ, Sieminski DJ, Katzel LI, Yataco A, Gardner AW. Improved walking economy in patients with peripheral arterial occlusive disease Med Sci Sports Exerc. 1997;29:1286-1290.

40. Bendermacher BL, Willigendael EM, Teijink JA, Prins MH. Supervised exercise therapy versus non-supervised exercise therapy for intermittent claudication. Cochrane database of systematic reviews (Online). 2006(2):CD005263.

41. Lee HL, Mehta T, Ray B, Heng MS, McCollum PT, Chetter IC. A non-randomised controlled trial of the clinical and cost effectiveness of a Supervised Exercise Programme for claudication. Eur J Vasc Endovasc Surg. 2007;33:202-207.

42. Imfeld S, Singer L, Degischer S, Aschwanden M, Thalhammer C, Labs KH, Jaeger KA. Quality of life improvement after hospital- based rehabilitation or home-based physical training in intermittent claudication. VASA. 2006:35:178-184.

43. Hirsch AT, Haskal Z, Hertzer NR, et al. ACC/AHA Guidelines for the management of patients with peripheral arterial diseases (lower extremity, renal, mesenteric and abdominal aortic): a collaborative report from the American Association for Vascular Surgery/Society for Vascular Surgery, Society for Cardiovascular Angiography and Interventions, Society for Interventional Radiology, Society for Vascular Medicine and Biology, and the American College of Cardiology/ American Heart Association Task Force on Practice Guidelines (Writing Committee to develop guidelines for the management of patients with peripherd arterial disease). Circulation.2005;21:e463-e654.
44. Willigendael EM, Bendermacher BL, van der Berg C, Welten RJ, Prins MH, Bie de RA, Teijink JA. The development and implementation of a regional network of physiotherapists for exercise therapy in patients with peripheral arterial disease, a preliminar report. BMC Heath Serv Res. 2005;5:49.

45. Jongert MWA, Hendriks HJM, Van Hoek J, et al. KNGF-richtliin Claudicatio Intermittens. Ned Tijdschr Fysiother 2003;Suppl:3-58. 
CHAPTER 2

Pocket Doppler and vascular laboratory equipment yield comparable results for ankle brachial index measurement 


\section{Abstract}

Background: The ankle brachial index (ABI) is a well-established tool for screening and diagnosis of peripheral arterial disease (PAD). In this study we assessed the validity of $\mathrm{ABI}$ determination using a pocket Doppler device compared with automatic vascular laboratory measurement in patients suspected of PAD.

Methods: Consecutive patients with symptoms of PAD referred for ABI measurement between December 2006 and August 2007 were included. Resting ABI was determined with a pocket Doppler, followed by ABI measurement with automatic vascular laboratory equipment, performed by an experienced vascular technician. The leg with the lowest ABI was used for analysis.

Results: From 99 patients the mean resting ABI was 0.80 measured with the pocket Doppler and 0.85 measured with vascular laboratory equipment. A Bland-Altman plot demonstrated great correspondence between the two methods. The mean difference between the two methods was $0.05(P<.001)$. Multivariate linear regression analysis showed no dependency of the difference on either the average measured $\mathrm{ABI}$ or affected or unaffected leg.

Conclusions: Since the small, albeit statistically significant, difference between the two methods is not clinically relevant, our study demonstrates that $\mathrm{ABI}$ measurements with pocket Doppler and vascular laboratory equipment yield comparable results and can replace each other. Results support the use of the pocket Doppler for screening of PAD, allowing initiation of cardiovascular risk factor management in primary care, provided that the equipment operator is experienced.

\section{Introduction}

The ankle brachial index (ABI) is useful in the diagnosis of peripheral arterial disease (PAD). With a sensitivity and specificity of $90 \%$ and $98 \%$, respectively, ABI is especially helpful in establishing lower extremity PAD. ${ }^{1,2}$ The ABI has become increasingly important as a screening tool for identification of patients with asymptomatic $P A D,{ }^{3}$ which is an independent marker for adverse cardiovascular outcome. ${ }^{4} \mathrm{~A}$ patient with a low $\mathrm{ABI}$ has a 5.5 -fold increased risk of cardiovascular death and a 2.5 -fold higher risk of coronary artery disease and of stroke. ${ }^{5}$ Current guidelines recommend initiation of secondary prevention measures in all patients with a screening $A B I$ value $<0.9$ and treatment of atherosclerosis risk factors. ${ }^{3}$ Given the importance of the $\mathrm{ABI}$ as a predictor of cardiovascular disease and mortality, ${ }^{6}$ accurate determination of the $\mathrm{ABI}$ is crucial.

Equipment used to measure arm and ankle pressures differs between the primary care setting, outpatient clinics, and the vascular laboratory setting. Arm and ankle pressures in primary care and in outpatient clinics are usually measured with a pocket Doppler device. In vascular laboratory settings, these measurements are performed with automatic vascular laboratory equipment. Although the pocket Doppler method is widely used, comparisons of this method with vascular laboratory equipment have been limited. A recent study compared the $\mathrm{ABI}$ measurements of 30 patients with both types of equipment.7 The pocket Doppler measurement was performed by a nurse, while the measurement in the vascular laboratory was performed by a vascular technologist, and the two health care providers had different levels of expertise in performing ABI measurements. The aim of this study was to compare pocket Doppler $\mathrm{ABI}$ measurements with automatic measurements performed in a vascular laboratory, with the procedures being performed by observers with equal levels of expertise and to determine if there are intrinsic differences in the results obtained with these two devices.

\section{Methods}

Ninety nine consecutive patients suspected of PAD who had been referred to the vascular laboratory of our hospital for an ABI measurement between December 2006 and August 2007 were included in this study. Informed consent was obtained and the study was approved by the medical ethical committee of the Atrium medical center Parkstad. For valid comparisons of ABI measurements performed by pocket Doppler and with laboratory equipment, both measurements were conducted on the same day in the vascular laboratory. For both methods, brachial pressures were measured bilaterally, and were repeated if the difference was $>10 \mathrm{~mm} \mathrm{Hg}$ between the two arms. Ankle pressures were determined with cuffs placed proximal to the malleoli. Following a 15 minute resting period, systolic blood pressures in the brachial, dorsal pedal, and posterior tibial arteries were determined in a supine position with a pocket Doppler device (Doppler MD2, Huntleigh Healthcare, Cardiff, United Kingdom) by a trained vascular laboratory professional. Brachial and ankle pressures were measured with a sphygmomanometer cuff which was inflated and deflated manually. The first audible signal of the first ventricular systole was used to identify the systolic blood pressure at each location. The ABI was calculated by dividing the highest systolic ankle pressure (either posterior tibial or dorsal pedal) in each leg by the highest systolic brachial pressure. ${ }^{8,10}$ Then, all measurements were repeated by a second vascular technician, blinded to the previous results, using laboratory equipment (VasoGuard System XP84 (1999), Scimed, Bristol, United Kingdom). Sphygmomanometer cuffs used to measure brachial and ankle pressures inflated and deflated automatically by pressing a button. The cut-off points of all arteries were defined as the systolic upstroke 
of the first arterial waveform. At the first characteristic arterial sound and at the simultaneous appearance of the first arterial waveform, the monitor screen was frozen, and the systolic blood pressure cut-off point was defined by precise retrospective positioning of an adjustable marker line (Table 1).

Table 1: Comparison of pocket Doppler and vascular laboratory measurement of brachial and ankle blood pressures

\begin{tabular}{|c|c|c|}
\hline & Pocket Doppler & $\begin{array}{c}\text { Vascular laboratory } \\
\text { equipment }\end{array}$ \\
\hline $\begin{array}{l}\text { Probe } \\
\text { Cuff inflation/deflation } \\
\text { Systolic blood pressure } \\
\text { cut-off point }\end{array}$ & \begin{tabular}{l}
\multicolumn{1}{c}{$8 \mathrm{MHz}$} \\
Manually \\
- $\quad$ Audible signal \\
- View the manometer at the \\
time of the first sound \\
Rapid response with \\
immediate determination of \\
the cut-off point
\end{tabular} & \begin{tabular}{ll}
\multicolumn{1}{c}{$8 \mathrm{MHz}$} \\
- & Automatically \\
- & Adjual and audible signal \\
& the monitor line placed on \\
- & Line is precisely \\
positioned retrospectively
\end{tabular} \\
\hline
\end{tabular}

\section{Statistic evaluation}

$\mathrm{ABI}$ measurements for each leg of the same patient are probably correlated since atherosclerosis is a generalised disease. Therefore, we used the lower ABI of both legs of each patient for analyses. The ABI values obtained from the pocket Doppler and from the vascular laboratory were averaged. The leg affected with PAD was defined as a leg with an $A B I<0.9$. Differences between measurements were assessed with a one-sample Student's t-test. Multivariate linear regression analysis assessed the dependency of the observed

difference between the two measurements and the average measured ABI for the affected and unaffected legs. Due to ethical considerations, intra arterial blood pressures were not performed and a Bland-Altman plot was used to visualise agreement between the two methods. ${ }^{9}$ Statistical analysis was performed with SPSS version 14.0 for Windows.

\section{Results}

Characteristics of the study population are presented in Table 2 . The mean age of the 99 participating patients was 65.0 years. Characteristics of the study population are presented in Table 2. In total, 56 legs were diagnosed with PAD, (23 right legs and 33 left legs). The mean $\mathrm{ABI}$ of the 99 patients was 0.80 (SD 0.25) as measured with the pocket Doppler and 0.85 (SD 0.25) as measured with vascular laboratory equipment. Subtraction of the pocket Doppler result from the automatic vascular laboratory equipment result yielded a mean difference of 0.05 (SD 0.09), a value that was statistically significant $(P<.001)$. Multivariate linear regression analyses showed no dependency of the difference on the average measured $\mathrm{ABI}(\mathrm{P}=.187)$ or whether the measurements were performed on affected or unaffected legs $(P=235)$. The two methods were compared by a Bland-Altman plot (Figure 1) which depicts the average ABI as determined by the two measurements across the difference between the pocket Doppler and the automatic $\mathrm{ABI}$ measurement for each leg. The Bland-Altman analysis confirmed that the data obtained by the two procedures was virtually the same.

\begin{tabular}{ll}
\hline Table 2: Clinical characteristics of the study population \\
\hline & Analysed population $\mathbf{n = 9 9}$ \\
\hline Men $-\mathrm{n}(\%)$ & $60(60.6)$ \\
Age (years) - mean (SD) & $65.0(12.2)$ \\
BMI - mean (SD) & $26.6(3.9)$ \\
Hypertension $-\mathrm{n}(\%)$ & $72(72.7)$ \\
Diabetes Mellitus $-\mathrm{n}(\%)$ & $27(27.3)$ \\
\hline
\end{tabular}

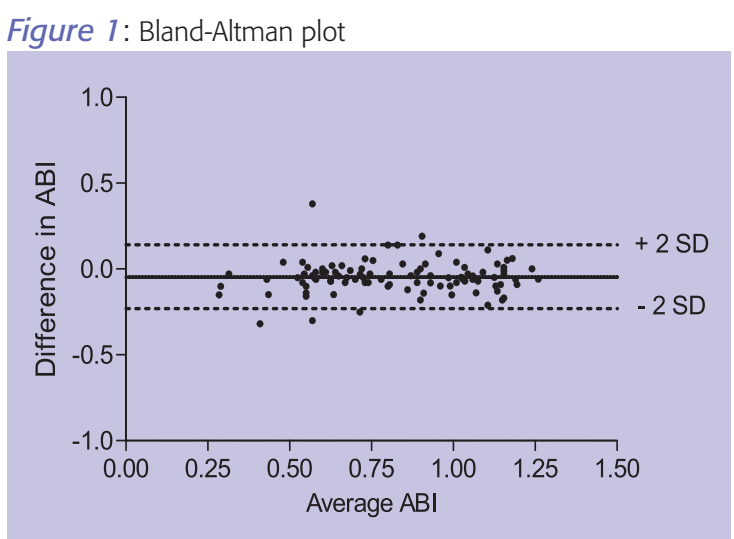

\section{Discussion}

This study shows that the ABI values determined by a simple pocket Doppler device and by automatic vascular laboratory equipment are interchangeable. In view of the importance of the $\mathrm{ABI}$ in detecting patients with atherosclerosis, our study supports the use of the easily accessible and applicable Doppler device for the screening and diagnosis of PAD, thus permitting the initiation of cardiovascular risk factor management in the primary care practice.

The good clinical interchangeability between ABI assessment with pocket Doppler and automatic vascular laboratory equipment was elegantly demonstrated by a BlandAltman plot. However, ABI, as measured by pocket Doppler, tended to be consistently lower, independent of the average of the measured $\mathrm{ABI}$ and whether measurements were obtained from the affected or the unaffected leg. Although the minor, albeit statistically significant, difference in $\mathrm{ABI}$ of 0.05 is not considered to be clinically relevant, ${ }^{10}$ it may be important in the epidemiological context. In larger studies, $A B I$ values close to the cut off point of 0.9 could influence the reported prevalence of $P A D,{ }^{11}$ and affect the determinations of sensitivity and specificity of the $A B I$ measurement for the identification of high risk patients. An additional study on a larger population is required to resolve this problem.

The small difference in $A B I$ between both methods may relate to the method of determination of systolic blood pressure cut-off points and cuff in- and deflation. With the pocket Doppler, systolic blood pressure is recorded from the sphygmomanometer simultaneously with the first audible signal, which can be influenced by human auditory limitations as well as by a slow response to the rapidly occurring audible signal. Most laboratory equipment automatically visualises the Doppler signal output with spectral analysis and displays the entire frequency and amplitude of the Dopple 
signal on the monitor. ${ }^{12}$ The screen is frozen as the first arterial waveform is displayed and is accompanied by the audible signal. Subsequently, an adjustable line is placed precisely at the systolic upstroke of the first arterial waveform. Other artefacts including slight movements of the hand holding the Doppler device during manual inflation and deflation, alterations in the position or angle of the device, and variations in the amount of pressure can affect the quality of the Doppler signal, and consequently the systolic blood pressure measurement. ${ }^{13}$

In general, screening for PAD by ABI and thus, screening for atherosclerosis in peripheral arteries of the leg as a reflection of generalised atherosclerosis is highly encouraged. However, we suggest that clinical judgement must be used in the interpretation of $A B I$ values determined by pocket Doppler. Diabetes or longstanding renal failure medial calcinosis could lead to calcified arteries which may be inadequately compressed by the sphygmomanometer cuff, leading to falsely elevated ankle pressures. Referral to a vascular laboratory for the measurement of systolic toe pressures or additional vascular imaging is essential for adequate determination of the vascular status of these patients. ${ }^{10}$

Furthermore, an experienced operator is mandatory for accurate determination of the $\mathrm{ABI}$, as previously indicated by the positive influence of experience and training on the reproducibility of the $A B I$ measurement. ${ }^{14,15}$ Ray et al. demonstrated that inexperienced doctors performed $\mathrm{ABI}$ measurements less reliably than their trained counterparts. ${ }^{16}$

In the present study, all vascular technicians were trained and experienced. Since the pocket Doppler method is highly operator dependent, it is particularly important that medical personnel is adequately trained in the acquisition of data using the pocket Doppler device so that $\mathrm{ABI}$ assessment can be widely applicable in primary care practice. However, the advised methods reported in the literature vary for the performance and the calculation of ABI measurement. ${ }^{10,12,17}$ Ideally, guidelines consistent with the method of measurement and calculation of the ABI should be established. Indeed, this instruction could be the basis for structured training programmes for medical personnel to develop this expertise. The present study shows that the pocket Doppler and automatic vascular laboratory equipment measurements of the ABI are interchangeable in patients suspected of PAD. The validity of pocket

Doppler $A B I$ measurement in a screening setting could be a subject of future studies.

\section{Conclusions}

Pocket Doppler assessment was demonstrated to be a practical tool for reliable and quick evaluation of the vascular status of a patient. This provides a useful tool for the investigation of patients with lower limb pain, and enables the targeted referral of patients with symptomatic PAD to the vascular specialist. Even more importantly, it introduces the opportunity for atherosclerosis screening and cardiovascular risk management in asymptomatic patients to reduce cardiovascular morbidity and mortality.

\section{Acknowledgements}

We would like to thank the vascular technicians of the non-invasive vascular lab for performing the $\mathrm{ABI}$ measurements.

\section{References}

01. Yao ST, Hobbs JT, Invine WT. Ankle systolic pressure measurements in arterial disease affecting the lower extremities. Br J Surg 1969 Sep;56:676-9.

02. Ouriel K, McDonnell AE, Metz CE, Zarins CK. Critical evaluation of stress testing in the diagnosis of peripheral vascular disease Surgery 1982;91:686-93.

03. Hirsch AT, Haskal Z, Hertzer NR, Bakal CW, Creager $\mathrm{MA}$, Halperin $\mathrm{J}$, et al. ACC/AHA Guidelines for the management of patients with peripheral arterial diseases (lower extremity renal mesenteric and abdominal aortic): a collaborative report from the American Association for Vascula Surgery/Society for Vascular Surgery, Society for Cardiovascular Angiography and Interventions, Society for Interventional Radiology, Society for Vascular Medicine and Biology, and the American College of Cardiology/American Heart Association Task Force on Practice Guidelines (Writing Committee to develop guidelines for the management of patients with peripheral arterial disease). Circulation 2005:21:e463-e654.

04. Fowkes FG, Murray GD, Butcher I, Heald CL, Lee $\mathrm{RJ}$, Chambless LE, et al. Ankle brachial index combined with Framingham Risk Score to predict cardiovascular events and mortality: a metanalysis. JAMA 2008; ;300:197-208

05. Doobay AV, Anand SS. Sensitivity and specificity of the ankle-brachial index to predict future cardiovascular outcomes: a systematic review. Arterioscler Thromb Vasc Biol 2005;25:1463-9.

06. Criqui MH, Langer RD, Fronek A, Feigelson HS, Klauber MR, McCann TJ, et al. Mortality over a period of 10 years in patients with peripheral arterial disease. New Engl J Med 1992;326:381-6.

07. Bonham PA, Cappuccio M, Hulsey T, Michel Y, Kelechi $T$, Jenkins $C$, et al. Are ankle and toe brachial indices (ABI-TBI) obtained by a pocket Doppler interchangeable with those obtained by standard laboratory equipment? J Wound Ostomy Continence Nurs 2007:34:35-44.
08. Atsma F, Bartelink ML, Grobbee DE, van der Schouw YT. Best reproducibility of the ankle-arm index was calculated using Doppler and dividing highest ankle pressure by highest arm pressure. J Clin Epidemiol 2005;58:1282-8

09. Bland JM, Altman DG. Statistical methods for assessing agreement between two methods of clinical measurement. Lancet 1986;1:307-10

10. Norgren L, Hiatt WR, Dormandy JA, Nehler MR, Harris KA Fowkes FG. Inter-Society Consensus for the Management of Peripheral Arterial Disease (TASC II). J Vasc Surg 2007;45 Suppl S:S5-67.

11. Lange SF, Trampisch HJ, Pittrow D, Darius H, Mahn M, Allenberg JR, et al. Profound differences of different methods for determination of the ankle brachial index on the prevalence estimate of peripheral arterial disease BMC Public Health 2007,7:147.

12. Bartelink ML SH, Boutens EE, Hooi JD, Kaiser V, Boomsma L. NHG-standaard perifeer arterieel vaatlijden (eerste herziening). Huisarts Wet 2003;46:848-58.

13. Cronenwett JL. The vascular laboratory. In: Rutherford RB (ed). Vascular surgery. 5 ed. Philadelphia: Saunders, 2000:127-39.

14. Matzke S, Franckena M, Alback A, Railo M, Lepantalo $M$. Ankle brachial index measurements in critical leg ischaemia-the influence of experience on reproducibility. Scand J Surg 2003;92:144-7.

15. Kaiser V, Kester AD, Stoffers HE, Kitslaar PJ, Knottnerus JA. The influence of experience on the reproducibility of the ankle-brachial systolic pressure ratio in peripheral arterial occlusive disease. Eur Vasc Endovasc Surg. 1999:18:25-9.

16. Ray SA, Srodon PD, Taylor RS, Dormandy JA. Reliability of anklebrachial pressure index measurement by junio doctors. Br J Surg 1994:81:188-90

17. Greenland P, Abrams J, Aurigemma GP, Bond MG, Clark IT, Criqui MH, et al. Prevention Conference V: Beyond secondary prevention: identifying the high-risk patient for primary prevention: noninvasive tests of atherosclerotic burden: Witing Group III. Girculation 2000; 101(1):E16-22. 
CHAPTER 3

Ankle

brachial

index

measurement

in primary care;

are we

doing it right?

Saskia P.A. Nicolaï

Lotte M. Kruidenier

Ellen V. Rouwet

Marie-Louise E.L. Bartelink

Martin H. Prins

Joep A.W. Teijink

Br J Gen Prac. 2009;59:422-7 


\section{Abstract}

Background: The reference standard for diagnosing PAD in primary care is the ankle brachial index $(\mathrm{ABI})$. Various methods to measure ankle and brachial blood pressures and to calculate the index are described.

Aim: To compare the $\mathrm{ABI}$ measurements performed in primary care with those performed in the vascular laboratory. Furthermore, an inventory was made of methods used to determine the $\mathrm{ABI}$ in primary care.

Design of Study: Cross-sectional study

Setting: Primary care practice and outpatient clinic

Methods: Consecutive patients suspected of PAD based on $\mathrm{ABI}$ assessment in primary care practices were included. The $A B I$ measurements were repeated in the vascular laboratory. Referring general practitioners were interviewed about method of measurement and calculation of the index. From each patient the leg with the lower $\mathrm{ABI}$ was used for analysis.

Results: Ninety nine patients of 45 primary care practices with a mean $\mathrm{ABI}$ of 0.80 (SD 0.27) were included. The mean ABI as measured in the vascular laboratory was 0.82 (SD 0.26). A Bland Altman plot demonstrated great variability between $A B$ measurements in primary care practice and the vascular laboratory. Both method of blood pressure measurements and method of calculating the ABI greatly differed between primary care practices.

Conclusion: This study demonstrates that the ABI is often not correctly determined in primary care practice. This phenomenon seems to be due to both inaccurate methods for blood pressure measurements as well as for calculation of the index. A guideline for determining the $\mathrm{ABI}$ with a hand-held Doppler and a training program seem necessary.

\section{Introduction}

General practitioners (GPs) play a key role in diagnosis and treatment of patients with peripheral arterial disease (PAD). As international guidelines recommend, the reference standard for diagnosing PAD in primary care is measurement of the ankle brachial index $(\mathrm{ABI})$. $\mathrm{An} A \mathrm{AI}<0.9$ indicates the presence of $\mathrm{PAD}$ in symptomatic patients as well as in asymptomatic patients. In addition, an $\mathrm{ABI}<0.9$ reflects the presence of generalised asymptomatic atherosclerotic disease, and its associated increased cardiovascular risk. ${ }^{1}$ There is an increased use of the ABI in primary care as an effect of the upcoming office-based cardiovascular screening and prevention programs, since current guidelines recommend to initiate secondary prevention of atherosclerotic disease in all patients with an $\mathrm{ABI}<0.9 .^{2}$ Moreover, reimbursement, one of the limitations of incorporating $A B I$ into daily practice ${ }^{3}$, was recently introduced in the Netherlands for $A B I$ measurement in primary care.

The $\mathrm{ABI}$ is a non-invasive, simple and inexpensive test with a good diagnostic performance if determined by well-trained professionals., ${ }^{4,5}$ However, reproducibility of the $A B I$ is dependent on training and experience of the observer, which may be difficult in primary care practice. Moreover, there are various ways to perform the measurement and to calculate the index. ${ }^{6-9}$ Arm pressures can be measured at one or two arms and ankle pressures at the posterior tibial and/or the dorsal pedal artery. In the situation that systolic pressures of two arms or two ankle arteries are measured the highest, average or lowest pressure can be used for calculating the index Theoretically, this results in at least 25 different possible combinations to calculate the ABI.

We aimed to compare the reported results of $\mathrm{ABI}$ measurements performed in primary care with those performed in the vascular laboratory, with attention to the method

used for the blood pressure measurement and calculation of the index. In addition, an inventory was made of the used techniques and ways to measure and calculate the ABI.

\section{Methods}

All consecutive patients suspected of symptomatic PAD based on their complaints accompanied with an $A B I$ measurement in primary care practice who were referred to the researchers' outpatient vascular clinic by their GP were included in this study. All GP practices performing ABI Doppler measurements from the region the Atrium medical center Parkstad were eligible for inclusion. Informed consent was obtained from all patients and the study was approved by the medical ethical committee of the Atrium medical center Parkstad. Informed consent was obtained from all patients; all referring GPs were informed about the ongoing study and informed consent was also obtained.

Methods of $A B I$ measurement in the primary care practices were determined by a questionnaire with respect to the resting period prior tot blood pressure measurements, method of ankle and brachial systolic blood pressure measurements, numerator and denominator for calculation of the $\mathrm{ABI}$, frequency of $\mathrm{ABI}$ assessment, and specialised training. Questionnaires were administered after inclusion of the patients. 
In all patients the $\mathrm{ABI}$ measurement was repeated in the vascular laboratory within a short period of time, varying between one and four weeks. Following a 15-minute resting period, systolic blood pressures in the brachial, dorsal pedal, and posterior tibial arteries were determined in a supine position with a hand-held pocket Doppler device (Doppler MD2, 8MHz, Huntleigh Healthcare, Cardiff, United Kingdom) by a trained vascular technician blinded for the primary care ABI. Brachial and ankle pressures were measured with $10 \mathrm{~cm}$-wide sphygmomanometer cuffs which were manually inflated and deflated. The first audible signal of the first ventricular systole was used to identify the systolic blood pressure at each location. Brachial pressures were measured bilaterally and were repeated if the difference was $>10 \mathrm{mmHg}$ between the two arms. Ankle pressures were determined with cuffs placed proximal to the malleoli. For each leg, the ABI was calculated by dividing the highest systolic ankle pressure (either posterior tibial or dorsal pedal) by the highest systolic pressure of both arms. ${ }^{7}$ The lower $\mathrm{ABI}$ of both legs of each patient was used for further analysis.

Analysis

A Bland-Altman plot is used to compare two clinical measurement techniques that each provide some error, i.e. there is no golden standard which provides the exact

information. ${ }^{10}$ The Bland-Altman plot is therefore used to visualise agreement between two measurement techniques. Because the true value is not known, on the horizontal axis the mean of both measurements is presented. On the vertical axis the difference between the two measurements is presented. A Bland-Altman plot explores a possible relationship between the measurement error and the true value, for example in this study a lack of agreement between the two methods with the lower or the higher ABIs can be visualised with this plot. To assess if there was a dependency of the difference between the two measurements on the average of the measurements univariate

linear regression analysis was used. Another way to demonstrate variability between the two $A B I$ measurements is by means of the coefficient of variation. To this end, the average of and the difference between the two $\mathrm{ABI}$ measurements were calculated for each patient. The coefficient of variation for the study population was calculated as the standard deviation of the differences between the two ABI measurements divided by the mean of the averages. Then, the coefficient of variation is quoted as percentage. A coefficient of variation $<10 \%$ to $15 \%$ is generally regarded as acceptable for clinica tests. Univariate linear regression analysis was performed to assess the dependency of who performed the measurements in primary care and the frequency of $A B I$ measurements in primary care on the accuracy of the readings. In addition, a cross tabulation was made using an $\mathrm{ABI}<0.9$ and an $\mathrm{ABI} \geq 0.9$ as measured in primary care and in the vascular laboratory. The kappa and its 95\% confidence interval (CI) was calculated. Statistical analysis was performed with SPSS version 15.0 for Windows.

\section{Results}

The vascular surgical department of the Atrium medical center Parkstad is a referra center for approximately $140 \mathrm{GPs}$. Ninety-nine patients were referred by 61 different GPs out of 46 primary care practices. Each GP referred a mean of 1.6 patients (SD 0.98). The mean $\mathrm{ABI}$ measured in primary care setting was 0.80 (SD $0.27,95 \% \mathrm{Cl}$ 0.75 to 0.86 ) and in the vascular laboratory 0.82 (SD $0.26,95 \% \mathrm{Cl} 0.76$ to 0.87 ). The mean difference between the two measurements was 0.02 (SD $0.24,95 \% \mathrm{Cl}-0.06$ to 0.03$)(P=.528)$. Agreement between the $A B I$ measurement in primary care and the $\mathrm{ABI}$ measurement performed in the vascular laboratory was visualised in a Bland-
Altman plot. (Figure 1) This plot depicts the difference between the measurement in primary care and the measurement in the vascular laboratory for each leg in relation to the average $\mathrm{ABI}$ as determined by the two measurements. Furthermore, the 'limits of agreement ${ }^{\prime}$ which are expressed as $\pm 2 \mathrm{SD}$ are given in the figure. In this study these intervals are wide (SD 0.24), reflecting the great variation of the differences. 10 Linear regression failed to show a relation for the difference of the measurements to the average of the measurements, indicating that the accuracy of the measurements is equal for all the $\mathrm{ABI}$ measurements (high and low). The coefficient of variation between both measurements was $29.6 \%$, meaning that there is considerable variation between the measurement in primary care and that in the vascular laboratory.

Figure 1: Average $\mathrm{ABI}$ as determined by the two measurements across the difference between the ABI measurements in primary care practice and the measurements in the vascular laboratory

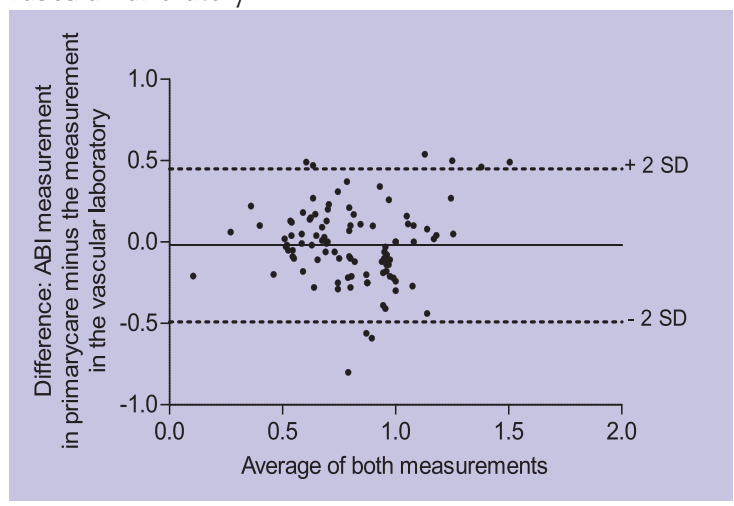

Table 1; Cross tabulation of the $\mathrm{ABI}$ measurements in primary care and in the vascular laboratory.

\begin{tabular}{llll}
\hline & \multicolumn{3}{c}{ Vascular laboratory } \\
\hline & & $A B \mid<0.9$ & $A B \mid \geq 0.9$ \\
Primary care & $A B \mid<0.9$ & 47 & 19 \\
& $A B \mid \geq 0.9$ & 9 & 24
\end{tabular}

The Bland Altman plot implies that neither measurement counts as a 'gold standard'. However, to demonstrate the clinical relevance of the discrepancy in $A B I$ measurements between primary practice and vascular lab, the data were also presented in a cross tabulation, classifying the results of the $A B I$ measurement as normal $(\geq 0.9)$ or as abnormal $(<0.9)$ (Table 1). The agreement between primary care and the vascular laboratory was 0.41 (kappa, 95\% Cl 0.22 to 0.59 ), indicating a poor agreement beyond change. In 19 out of 66 patients referred with an $A B I$ measurement $<0.9$ in primary care practice, the $\mathrm{ABI}$ measurement was $\geq 0.9$ in the vascular laboratory. Assuming the vascular laboratory reading to be the gold standard, in these patients the diagnosis of PAD was rejected. 
Table 2: Methods used in primary care practice to measure ankle and brachial blood pressures

\begin{tabular}{lll} 
Part of ABI method & & $\begin{array}{l}\text { Primary care practices } \\
(\mathrm{n}=45)\end{array}$ \\
\hline Resting period & $\geq 10$ minutes & $21(47 \%)$ \\
& $<10$ minutes & $13(29 \%)$ \\
Brachial systolic pressure & No rest & $10(22 \%)$ \\
& Both arms & $41(91 \%)$ \\
Device for blood pressure & One arm & $4(9 \%)$ \\
measurement & Doppler device & $28(62 \%)$ \\
& & \\
Ankle systolic pressure & Korotkoff method & $16(36 \%)$ \\
& Automatic device & $1(2 \%)$ \\
& Dorsal pedal and posterior & $23(51 \%)$ \\
& tibial artery & \\
& Posterior tibial artery & $15(33 \%)$ \\
& Dorsal pedal artery & $6(13 \%)$ \\
& One unknown artery & $1(2 \%)$ \\
\hline
\end{tabular}

$A B I$ denotes ankle brachial index

Methods of $A B I$ determination in primary care practices

Method of ABI measurement was evaluated by telephonic questionnaire. Forty-five out of 46 practices participated. ABI was determined by GPs in 8 practices, and by a non-doctor in 37 practices. In 26 practices, the professional had followed a structured training for $\mathrm{ABI}$ assessment. Linear regression did not show a difference in accuracy of $A B I$ measurement between $G P s$ and non-doctors $(P=.213)$. Furthermore, linear regression analysis did not reveal a difference between primary care practices performing an $\mathrm{ABI}$ measurement more or less than once a week $(P=.524)$. The questionnaire showed that 34 out of 45 primary care practices planned a period of rest before the measurement was started (Table 2). This period of rest varied between 5 and 20 minutes (mean 8.9 minutes, SD 4.2 minutes). In 41 primary care practices bilateral systolic blood pressure of the arms was performed, and in the majority (28 practices) this was performed with a pocket Doppler device and a sphygmomanometer cuff. In the other cases, the systolic blood pressure was measured with a stethoscope and a sphygmomanometer (16 practices) or an oscillometric automatic device (one practice). In 23 practices, the systolic blood pressures of the dorsal pedal and the posterior tibial artery were determined in all patients, independent of their complaints. In the remaining 22 practices the systolic blood pressure of one pedal artery was investigated - this was the tibial posterior or dorsal pedal artery in 15 and 6 practices, respectively. Eight different methods were used for calculation of the $A B I$ in primary care practices. In primary care practices measuring the systolic blood pressure in two pedal arteries, the majority divided the highest ankle pressure by the highest arm pressure. However, other calculations used the mean of both ankle pressures and divided the right ankle pressure by the right arm pressure, and the left ankle pressure by the left arm pressure. Eight out of 45 primary care practices measured and calculated the ABI according to the method used in the vascular laboratory.

\section{Discussion}

Summary of main findings

This study shows that, overall, ABI measurements are comparable between primary care practices and the vascular laboratory. However, for the individual patient the ABI as assessed in the GP's office may vary significantly from that assessed in the vascular laboratory. This may be related to the method of ankle and brachial blood pressure measurements as well as the calculation of the index.

Strengths and limitations of the study

In this study, blood pressure measurements were performed using a hand-held pocket Doppler device. The pocket Doppler is a relatively cheap and easy to use device with a good inter- and intra-observer variability for $A B I$ assessment, at least in trained operators. ${ }^{4,} 11,12$ It has been shown that the reproducibility ABI assessment by pocket Doppler may be dependent on the level of experience of the operator. ${ }^{4,5}$ The most important limitations to incorporate $\mathrm{ABI}$ measurements in primary care are time constraints while an $\mathrm{ABI}$ measurement takes 12 to 15 minutes,, 13 reimbursement, and staff availability. ${ }^{3}$ Furthermore, in patients with $P A D$, there is variability in $A B I$ measurements that is attributable to biological factors and can differ between days. Baker et al. showed that the ABI must change at least 0.15 before this can be considered significant..$^{14}$

In this study, the second measurement was performed one to four weeks later than the measurement in primary care, which could at least partially explain the variability found between the measurements in primary care and in the vascular laboratory.

All patients included in this study were referred to the vascular laboratory for evaluation of suspected symptomatic PAD. Since the national guideline suggests that GPs can initiate treatment of patients by exercise therapy and cardiovascular risk management ${ }^{15}$ it is not possible to say how many patients from these primary care practices were not referred to the vascular laboratory for an additional ABI measurement. Although it would be informative to investigate the number of missed diagnosis, the aim of the current study was to investigate the difference in $\mathrm{ABI}$ measurement as determined in primary care practice and in vascular laboratory, not to assess patient outcome based on $\mathrm{ABI}$ measurement in primary care practice.. It is possible that the results of this study can not directly be translated to the 'healthy' screening population in general practice. However, this should have no influence on the method used to determine and calculate the $\mathrm{ABI}$ in primary care.

Comparison with existing literature

In this study, great variability is demonstrated between the $A B I$ measurement in primary care and in the vascular laboratory. Another recent study shows that $A B I$ measurements by GPs and nurses are highly reproducible. ${ }^{16}$ However, reproducibility of the ABI is dependent on the experience of the observer, ${ }^{4,5,17}$ and in the aforementioned study a limited number of selected GPs received a specialised training before starting the study. This seems not to reflect the current situation in primary care.

National and international guidelines recommend the use of the $A B I$ in routine screening for atherosclerosis. ${ }^{2,7}$ Substantial prevalence rates of asymptomatic PAD in the older population in primary care, with at least one vascular risk factor, were found. ${ }^{18-20} \mathrm{~A}$ recent meta-analysis showed that a low $\mathrm{ABI}$ in a healthy screening population was associated with approximately twice the 10-year total mortality, cardiovascular mortality, and major coronary events.' Current guidelines recommend initiation of secondary prevention of 
atherosclerotic disease in all patients with an $A B I<0.9 .{ }^{2}$ Given the importance of the $A B I$ in the initial diagnosis of PAD, and as a predictor of cardiovascular morbidity and mortality in general, accurate determination of the ABI is crucial for determining the cardiovascular risk profile and successful cardiovascular risk factor management.

Surprisingly, however, these guidelines do not provide step-by-step information about the correct method of ABI assessment. $2,7,15$ Moreover, different modes of determination and calculation of the $\mathrm{ABI}$ are used and advised.6,8 Klein et al. found 39 different ways to calculate the $\mathrm{ABI}{ }^{8} \mathrm{~A}$ recently published literature analysis that evaluated the methodology of $\mathrm{ABI}$ determination in 100 random publications demonstrated great variability with respect to the methods of ankle and brachial blood pressure measurements as well as calculation of the index. ${ }^{8}$ This large variety in calculation of the $\mathrm{ABI}$ can result in different reports of PAD prevalence. ${ }^{21,22}$

Recently in the literature, attention has been paid to alternative methods to detect or exclude patients with PAD. However, the Edinburgh Claudication Questionnaire has an inadequate diagnostic value for detecting patients with PAD. ${ }^{23}$ Furthermore, pulse palpation has been shown by some authors to not be sensitive to detection of PAD ${ }^{24,25}$ however, another study has suggested it is possible to exclude PAD by pulse palpation. ${ }^{26}$ Easier methods to measure the ABI are also described in the literature; however, results concerning the reliability of automated oscillometry to facilitate the $\mathrm{ABI}$ measurement in clinical practice are contradictory.25, 27-29

Implications for further research or clinical practice

This study demonstrates that the $\mathrm{ABI}$ is often not correctly determined in primary care practice. This phenomenon seems to be due to inaccurate methods for ankle and brachial blood pressure measurements and calculation of the index. A clear stepby-step guideline for determining the $\mathrm{ABI}$ with a hand-held Doppler and a training program seem necessary.

\section{Acknowledgements}

We would like to thank the vascular technicians who performed the ankle brachial index measurements in the vascular laboratory.

\section{References}

1. Fowkes FG, Murray GD, Butcher I, Heald CL, Lee RJ, Chambless LE, et al. Ankle brachial index combined with Framingham Risk Score to predict cardiovascular events and mortality: a metaanalysis. JAMA. 2008;300:197-208.

2. Hirsch AT, Haskal Z, Hertzer NR, Bakal CW, Creage MA, Halperin JL, et al. ACC/AHA Guidelines for the management of patients with peripheral arterial diseases (lower extremity, renal, mesenteric and abdominal aortic): a collaborative report from the American Association for Vascular Surgery/Society for Vascular Surgery, Society for Cardiovascular Angiography and Interventions, Society for Interventional Radiology, Society for Vascular Medicine and Biology, and the American College of Cardiology/American Heart Association Task Force on Practice Guidelines (Writing Committee to develop guidelines for the management of patients with peripheral arterial disease). Circulation. 2005;21:e463-e654.

3. Mohler ER, 3rd, Treat-Jacobson D, Reilly MP, Cunningham KE, Miani M, Criqui MH, et al. Utility and barriers to performance of the anklebrachial index in primary care practice. Vasc Med. 2004;9:253-260

4. Kaiser V, Kester AD, Stoffers HE, Kitslaar PI, Knottnerus JA. The influence of experience on he reproducibility of the ankle-brachial systolic pressure ratio in peripheral arterial occlusive disease. Eur J Vasc Endovasc Surg. 1999;18:25-29.

5. Matzke S, Franckena M, Alback A, Railo M Lepantalo M. Ankle brachial index measurements in critical leg ischaemia-the influence of experience on reproducibility. Scand I Surs. 2003;92:144-147.

6. Greenland P, Abrams 1 Aurigemma GP, Bond MC, Clark LT, Criqui MH, et al. Prevention Conference $V$ : Beyond secondary prevention: identifying the highrisk patient for primary prevention: noninvasive ests of atherosclerotic burden: Writing Group III. Circulation. 2000;101:E16-22.
7. Norgren L, Hiatt WR, Dormandy JA, Nehler MR, Harris KA, Fowkes FG. Inter-Society Consensus for the Management of Peripheral Arterial Disease (TASC II). J Vasc Surg. 2007;45 Suppl S:S5-67.

8. Klein S, Hage JJ. Measurement, calculation, and normal range of the ankle-arm index: a bibliometric analysis and recommendation for standardization. Ann Vasc Surg. 2006;20:282-292.

9. Caruana MF, Bradbury AW, Adam DJ. The validity, reliability, reproducibility and extended utility of ankle to brachial pressure index in current vascular surgical practice. Eur J Vasc Endovasc Surg. 2005;29:443-451.

10. Bland JM, Altman DG. Statistical methods for assessing agreement between two methods of clinical measurement Lancet. 1986:1:307-310.

11. Aboyans V, Lacroix P, Lebourdon A, Preux PM, Ferrieres J, Laskar $\mathrm{M}$. The intra- and interobserver variability of ankle-arm blood pressure index according to its mode of calculation. J Clin Epidemiol. 2003;56:215-220.

12. Stoffers $H E$, Kester $A D$, Kaiser V, Rinkens $P E$, Kitslaar PJ, Knottnerus JA. The diagnostic value of the measurement of the ankle-brachial systolic pressure index in primary health care. J Clin Epidemiol. 1996;49:1401-1405.

13. Bendermacher BL, Teijink JA, Willigendae EM, Bartelink ML, Peters RJ, Boiten J, et al. Applicability of the ankle brachial index measurement as screening device in general practice for high cardiovascular risk. Peripheral arterial disease; screening, diagnosis, and conservative treatment. Heerlen; 2007

14. Baker JD, Dix DE, Variability of Doppler ankle pressures with arterial occlusive disease: an evaluation of ankle index and brachial-ankle pressure gradient. Surgery. 1981;89:134-137.

15. Bartelink ML SH, Boutens EJ, Hooi JD, Kaiser V, Boomsma L. NHG-standaard perifeer arteriee vaatlijden. Huisarts Wet. 2003:46:848-858. 
16. Holland-Letz $\mathrm{T}$, Endres HG, Biedermann $\mathrm{S}$, Mahn $M$, Kunert I, Groh S, et al. Reproducibility and reliability of the ankle-brachial index as assessed by vascular experts, family physicians and nurses. Vasc Med. 2007;12:105-112.

17. Ray SA, Srodon PD, Taylor RS, Dormandy JA. Reliability of ankle:brachial pressure index measurement by junior doctors. Br I Surg. 1994;81:188-190.

18. Hirsch AT, Criqui MH, Treat-Jacobson D, Regensteiner JG, Creager MA, Olin JW, et al. Peripheral arterial disease detection, awareness, and treatment in primary care. JAMA. 2001;286:1317-1324

19. Diehm C, Lange S, Darius H, Pittrow D, van Stritzky B, Tepohl G, et al. Association of low ankle brachial index with high mortality in primary care. Eur Heart J. 2006;27:1743-1749.

20. Bendermacher BL, Teijink JA, Willigendael EM, Bartelink ML, Peters RJ, de Bie RA, et al. A clinical prediction model for the presence of peripheral arterial disease--the benefit of screening individuals before initiation of measurement of the ankle-brachial index: an observational study. Vasc Med. 2007;12:5-1

21. McDermott MM, Criqui MH, Liu K, Guralnik JM, Greenland P, Martin GJ, et al. Lower ankle/ brachial index, as calculated by averaging the dorsalis pedis and posterior tibial arterial pressures, and association with leg functioning in peripheral arterial disease. J Vasc Surg. 2000;32:1164-1171.

22. Lange $\mathrm{SF}$, Trampisch $\mathrm{HJ}$, Pittrow $\mathrm{D}$, Darius $\mathrm{H}$, Mahn M, Allenberg JR, et al. Profound influence of different methods for determination of the ankle brachial index on the prevalence estimate of peripheral arterial disease. BMC Public Health. 2007;7:147.
23. Bendermacher BL, Teijink JA, Willigendael EM, Bartelink ML, Buller HR, Peters RJ, et Symptomatic peripheral arterial disease: the value of a validated questionnaire and a clinical decision rule. Br J Gen Pract. 2006;56:932-937.

24. Collins TC, Suarez-Almazor M, Peterson NJ. An absent pulse is not sensitive for the early detection of peripheral arterial disease.

Fam Med. 2006;38:38-42.

25. Aboyans V, Lacroix P, Doucet S, Preux PM, Criqui $\mathrm{MH}$, Laskar M. Diagnosis of peripheral arterial disease in general practice: can the ankle-brachia index be measured either by pulse palpation or an automatic blood pressure device? Int I Clin Pract. 2008;62:1001-1007.

26. Migliacci R, Nasorri R, Ricciarini P, Gresele P. Ankle-brachial index measured by palpation for the diagnosis of peripheral arterial disease. Fam Pract. 2008;25:228-232.

27. Beckman JA, Higgins CO, Gerhard-Herman M. Automated oscillometric determination of the ankle-brachial index provides accuracy necessary for office practice. Hypertension. 2006;47:35-38.

28. Gardner AW, Montgomery PS. Comparison of three blood pressure methods used for determining ankle/brachial index in patients with intermittent claudication. Angiology. 1998:49:723-728

29. MacDougall AM, Tandon V, Wilson MP, Wilson TW. Oscillometric measurement of ankle-brachial index. Can J Cardiol. 2008;24:49-51.

\section{CHAPTER 4}

Rellability

of treadmill

testing in

peripheral

arterial disease;

a meta-regression analysis
Saskia P.A. Nicolaï
Wolfgang Viechtbauer
Lotte M. Kruidenier
Math J.J.M. Candel
Martin H. Prins
Joep A.W. Teijink

J Vasc Surg. 2009; 2009;50:322-9 


\section{Abstract}

Objective: A meta-regression analysis was conducted to identify the most reliable treadmill protocol for the assessment of patients with peripheral arterial disease.

Background: Treadmill testing is the main assessment method to evaluate walking ability in patients with peripheral arterial disease in clinical studies. Reported treadmill protocols are continuous $(C)$ and graded $(G)$ protocols and outcome measurements are initial claudication distance (ICD) and absolute claudication distance (ACD). Variety in protocols might hamper the ability to compare results of different studies. Ideally, future studies should use a protocol with highest reliability.

Methods: We searched Pubmed and EMBASE (until February 2008) and we hand searched the reference lists. Trials assessing reliability of treadmill testing were identified. Inclusion criteria were the use of a C- or G-protocol, repetition of this protocol, and a retrievable intraclass correlation coefficient (ICC). We identified 8 studies in which 658 patients were included.

Results: For ICD, the estimated reliabilities of the C- and G-protocol (as assessed by the ICC) were 0.85 (95\% Cl: 0.82 to 0.88 ) and 0.83 (95\% Cl: 0.80 to 0.85 ), respectively, without dependency of the reliability on velocity or grade. For $A C D$, the reliability was significantly better for the G-protocol $(0.95,95 \% \mathrm{Cl}: 0.94$ to 0.96$)$ than for the C-protocol. Moreover, the reliability of the C-protocol was dependent on grade of the treadmill (0\%, 10\%, and 12\%) with a mean ICC of 0.76 (95\% Cl: 0.54 to 0.88), 0.89 (95\% Cl: 0.86 to 0.91), and 0.91 ( $95 \% \mathrm{Cl} 0.88$ to 0.92 ), respectively.

Conclusions: Treadmill assessment has the highest reliability when using a G-protoco together with the $A C D$ as the primary outcome measure.

\section{Introduction}

Characteristically, in patients with symptomatic peripheral arterial disease (PAD), pain starts during walking and disappears in rest. Walking ability as assessed by a treadmill test is usually the primary endpoint in clinical trials of treatments for patients with PAD. Results of treadmill testing are usually expressed as the initial claudication distance (ICD; the moment of the beginning of claudication pain) and/or the absolute claudication distance (ACD; the moment the test has to stop due to the maximal level of bearable claudication pain). Although

treadmill testing has face validity for daily walking ability, there are several limitations. While there is a relationship between daily walking ability and objective criteria for the severity of atherosclerotic lesions in the arteries of the legs (eg, the angiographic extent of the disease' and the subjective, functional walking limitation for the patient ${ }^{2-4}$ ), there is large variation. However, although the ability of treadmill testing to diagnose PAD or to classify functional severity of PAD is limited, the effects of treatment in properly designed randomized controlled trials can be evaluated by comparing treadmill results before and after treatment.

A wide range of treadmill protocols are used to assess walking ability of patients with PAD. The traditional test uses a constant speed and incline (continuous test or C-test) during the entire assessment phase. Typically, the speed is set to some value between 2 and $4 \mathrm{~km} / \mathrm{h}$ and the gradient to either $0 \%, 10 \%$, or $12 \%$. However, due to a wide range of walking distances in patients with PAD, it is usually difficult to select one continuous protocol (C-protocol) to assess all patients. Furthermore, the high initial workload of the C-test may not be tolerated by patients with severe PAD.

In the seventies a graded treadmill test (G-test) was developed in cardiology to evaluate suspected ischaemic heart disease. ${ }^{5}$ Velocity of the treadmill is constant during this test, but a gradual increase in slope of the treadmill leads to a gradual increase in metabolic demand. Several graded treadmill protocols (G-protocol) have also been developed for PAD. Two frequently used G-protocols by Hiatt et al. and Gardner et al. both use a constant speed of $3.2 \mathrm{~km} / \mathrm{h}$. However, the Hiatt protocol starts with $0 \%$ incline with $3.5 \%$ increase every three minutes ${ }^{6}$ while the Gardner protocol starts with $0 \%$ and increments with $2 \%$ every two minutes.

C- and G-protocols with different speeds and inclines of the treadmill result in varying workloads, hampering adequate comparison of studies (Figure 1). Ideally, future studies should use a treadmill protocol that has the highest reliability. Hence, we performed a meta-analysis to compare the reliability of different treadmill protocols.

\section{Methods}

Search strategy

A search of all articles related to reliability of treadmill testing in peripheral disease was performed. To include all available evidence, we searched Pubmed and EMBASE (until February 2008) for relevant studies. The following search terms were used: 'treadmill test' or 'exercise test' or 'protocol' and 'reproducibility' or 'reliability' and 'peripheral arterial disease' or 'intermittent claudication' or 'peripheral occlusive disease'. In addition to the electronic search, the reference lists of selected articles were reviewed and cross-references were examined. There was no restriction on language of publication. 
Figure 1a: Workloads for different C- and G-protocols expressed as cumulative kilocalories for person of $70 \mathrm{~kg}$.

Figure $1 \mathrm{~b}$ : Workloads for the G-protocols included in the analysis.

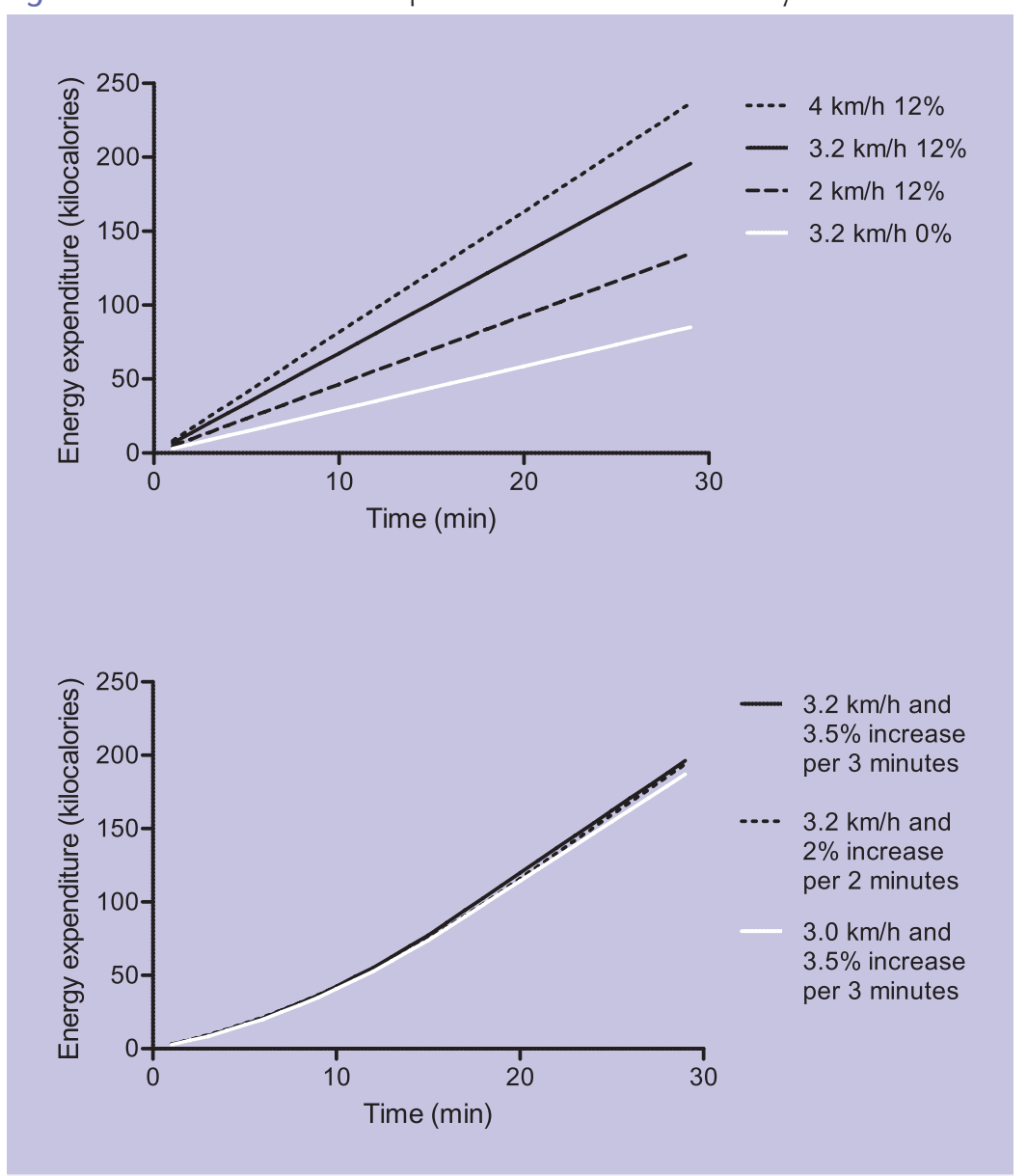

Selection of articles

Two authors (S.N. and L.K.) selected eligible articles evaluating reliability of treadmill testing by performing a repeated C- or G- test in patients with PAD. Discrepancies were resolved by discussion, or, if this failed, by consulting W.V. and M.P. Studies were excluded if the study population did not suffer from PAD or if walking ability was not quantified by treadmill testing. Eligible treadmill protocols were C-protocols, defined as protocols with the treadmill set to a fixed speed and incline, and G-protocols, defined as protocols with a fixed treadmill velocity with an increase in inclination at specified time intervals. Moreover, studies were excluded if the treadmill protocol was not repeated within 3 weeks. We chose for a limited period of time to diminish the effects of the natural development of the disease. Finally, studies were excluded if reliability was not presented as an intraclass correlation coefficient (ICC) or the ICC could not be calculated from the study's data or data provided by the author(s) of the study. Two reviewers (S.N. and M.P.) independently applied the inclusion criteria to select the relevant articles. In case of any disagreement, the problem was solved by discussion.

\section{Data extraction}

Data of included studies were extracted by two reviewers (S.N. and W.V.). The following information was sought from each study: number of patients, type of protocol (C- and/ or G-protocol, velocity and incline), mean walking distances (ICD and ACD), and the intraclass correlation coefficient (ICC) for each walking distance measure.

Walking distances are defined as the ICD, corresponding to the beginning of the claudication pain, and the $A C D$, corresponding to the moment the test has to stop due to the maximal level of bearable claudication pain. Written data were preferred whenever possible. If written data were not available, then walking distances with standard deviations were read out of figures. Furthermore, if the standard deviation was not provided, this was calculated from the standard error of the mean and the number of patients.

Based on the available data, it was decided to obtain ICC(1) values from the articles or through contact with the authors. The ICC $(1)$ is one out of several different intraclass correlation coefficients that have been developed and described in literature.

In general, ICCS are measures of agreement or consistency between measurements (or ratings). ICCs are particularly useful when more than two assessments have been made on a group of subjects (as is frequently the case in studies examining the reliability of treadmill protocols). For example, while three different pair wise correlation coefficients could be calculated based on three assessments of the ICD (or ACD) in a single group of patients, an ICC for the same data would provide an overall measure of the testretest reliability of the three assessments. For example, suppose the ICD values of three patients assessed at three occasions are equal to $126 \mathrm{~m}, 132 \mathrm{~m}, 136 \mathrm{~m}$ at the first, $130 \mathrm{~m}$ $128 \mathrm{~m}, 136 \mathrm{~m}$ at the second, and $126 \mathrm{~m}, 134 \mathrm{~m}, 134 \mathrm{~m}$ at the third assessment. The three pair wise correlations between the assessments are equal to 0.64, 0.92, and 0.28, yielding three rather different test-retest correlations. On the other hand, the ICC(1) for these data is equal to 0.73 .

Moreover, the ICC(1) is a measure of absolute agreement and therefore sensitive to changes in the average walking distance over time. For example, suppose the ICD values are equal to $126 \mathrm{~m}, 132 \mathrm{~m}, 136 \mathrm{~m}$ at the first, $134 \mathrm{~m}, 140 \mathrm{~m}, 144 \mathrm{~m}$ at the second, and $136 \mathrm{~m}, 142 \mathrm{~m}, 146 \mathrm{~m}$ at the third assessment. Then all three pair wise correlations are equal to 1 , while the ICC $(1)$ is equal to 0.36 , suggesting a much lower absolute agreement in the measurements over time.

In general, the ICC(1) is equal to the variability in the walking distances between patients divided by the total amount of variability in the data (which is equal to the variability between patients plus the variability in the walking distance within patients). The ICC(1) value can be easily estimated from an analysis of variance (ANOVA) with patients included as the only factor in the model. Based on the ANOVA table, we can calculate the ICC (1) with $I C C(1)=\left(M S_{B}-M S_{w}\right) \mid\left(M S_{B}+(m-1) M S_{w}\right)$, where $M S_{B}$ is the mean square corresponding to the patient factor, $\mathrm{MS}_{w}$ is the mean square error, and $\mathrm{m}$ denotes the number of assessment occasions. 
Data analysis

Since the distribution of ICC values is quite skewed (especially when the values are close to 1), all ICC(1) values were first converted with Fisher's variance stabilizing

transformation for intraclass correlation coefficients, ${ }^{9}$ which is given by

$y=0.5 \operatorname{In}[(1+(m-1) I C C(1)) /(1-I C C(1))]$. The sampling variance of the transformed values can then be approximated quite accurately with $v=m /[(2(m-1)(n-2)]$, where $n$ denotes the number of patients included in the study. Since four studies allowed the computation of multiple ICC(1) values from the same sample, it was also necessary to calculate the covariance between the dependent ICC(1) values. The equation for the covariance is given by Donner and Zou..$^{10} \mathrm{~A}$ fixed-effects meta-regression analysis was then performed to evaluate the influence of velocity, grade, and type of protocol (C- or G-protocol) on the reliability of treadmill testing. The potential moderators were examined separately and in combination. Estimated values and confidence interval $(\mathrm{Cl})$ bounds based on the models were back-transformed to estimated ICC(1) values with corresponding $\mathrm{Cl}$ bounds for easier interpretation. Tests for (residual) heterogeneity were conducted with the $\mathrm{Q}$-test. All tests were conducted with $a=05$.

\section{Results}

Identification of eligible studies

In total the electronic and manual literature search identified 43 potentially eligible articles on title. After evaluation, articles presenting an overview of the literature ${ }^{11,12}$ or letters to the editor ${ }^{13}$ and studies not performing treadmill testing in PAD patients ${ }^{14}$, 15 or not performing a treadmill test at all16 were excluded. Furthermore, studies using non eligible treadmill protocols, individualized per patient were excluded. ${ }^{17-20}$ Twelve studies were excluded because of the lack of repetition of an initial C- or G-test.3, 18, 21-30 In four studies the time between the treadmill tests exceeded our preset limit.,31-33 Finally, in nine studies, the ICC was not available and it was not possible to determine the ICC from the given data. ${ }^{6,3441}$ Hence eight studies could be included. In total, these studies reported 29 different ICCS.

\section{Study characteristics}

The 8 studies included a total of 658 patients. To determine reliability, four studies repeated one protocol. ${ }^{22-45}$ The other four studies compared more treadmill protocols within the same group of patients. ${ }^{46-49}$ Both C- and G-protocols were tested. The majority used a velocity of $3.2 \mathrm{~km} /$ hour, but also 2, 3 and $4 \mathrm{~km} /$ hour were used. Within a period of three weeks, all tests were repeated 2 or 3 times. Baseline characteristics of the included studies are described in Table 1.

Reliability of different protocols

In Table 2 the mean walking distances (ICD and ACD) are presented for all included studies as well as the reported or calculated ICC(1) values. The estimated reliabilities of the $\mathrm{C}$ - and G-protocol for the ICD, as assessed by the ICC(1), were 0.85 (95\% Cl: 0.82 to 0.88$)$ and 0.83 (95\% Cl: 0.80 to 0.85 ), respectively. The difference in the estimated ICC(1) values between the two protocols was not statistically significant $(z=1.40, P=.16)$. For the ICD there was no dependency of the reliability on velocity $(z=0.01, P=.99)$ or grade $(z=1.38, P=$ .17). In fact, no heterogeneity was detected within these data $(Q=15.71, d f=13, P=.27)$.

For the ACD the reliability, as assessed by the ICC ( 1 ), was significantly better $(z=5.01, P<$ $.001)$ for the G-protocol $(0.95,95 \%$ Cl: 0.94 to 0.96) than for the C-protocol $(0.90,95 \%$ $\mathrm{Cl}: 0.88$ to 0.92$)$. Furthermore, the reliability of the $\mathrm{C}$-protocol did not depend on velocity ( $\mathrm{z}=$ $1.34, \mathrm{P}=.18)$, but did depend on the grade $(z=2.55, \mathrm{P}=.01)$ of the treadmill protocol, with

\begin{tabular}{|c|c|c|c|c|c|c|}
\hline \multirow[t]{2}{*}{ study with year } & \multirow{2}{*}{$\begin{array}{l}\text { number } \\
\text { of patients }\end{array}$} & \multicolumn{3}{|c|}{ treadmill protocol } & \multirow{2}{*}{$\begin{array}{l}\text { number of } \\
\text { assessments }\end{array}$} & \multirow{2}{*}{$\begin{array}{l}\text { time between } \\
\text { assessments }\end{array}$} \\
\hline & & $\begin{array}{l}\text { Velocity } \\
(\mathrm{km} / \mathrm{h})\end{array}$ & $\begin{array}{l}\text { Grade } \\
(\%)\end{array}$ & $C / G$ & & \\
\hline Cachovan et al. & 50 & 3.0 & 12 & C & 3 & All assessments \\
\hline 1999 & & 3.0 & & GI & 3 & in 1 week \\
\hline Degischer et al. & 15 & 2.0 & 12 & C & 2 & All assessments \\
\hline \multirow[t]{4}{*}{2002} & & 3.2 & 0 & C & 2 & in \\
\hline & & 3.2 & 12 & C & 2 & 3 weeks \\
\hline & & 4.0 & 0 & C & 2 & \\
\hline & & 4.0 & 12 & C & 2 & \\
\hline Chaudhry et al. & 14 & 3.2 & 10 & $C$ & 2 & 2-7 days \\
\hline 1997 & & 3.2 & & G1 & 2 & \\
\hline Gardner et al. & 10 & 3.2 & 12 & C & 3 & 1 week \\
\hline 1991 & & 3.2 & & $\mathrm{G} 2$ & 3 & \\
\hline Gardner 1997 & 30 & 3.2 & & $\mathrm{G} 2$ & 3 & 1 week \\
\hline Labs et al. 1999 & 330 & 3.2 & & $\mathrm{G} 2$ & 3 & 1 week \\
\hline Labs et al. 1998 & 154 & 3.2 & 12 & $c$ & 2 & On the same da) \\
\hline $\begin{array}{l}\text { Zwierska et al. } \\
2004\end{array}$ & 55 & 3.2 & 12 & C & 3 & $\begin{array}{l}\text { Short period of } \\
\text { time }\end{array}$ \\
\hline
\end{tabular}

C/G indicates continuous protocol or graded protocol; $G 1$, graded protocol 1 with an increase in grade of 3.5\% every 3 minutes to a maximum of 17.5\%; G2, graded protocol 2 with an increase in grade of $2 \%$ every 2 minutes to a maximum of $18 \%$.

an estimated ICC(1) of 0.76 (95\% Cl: 0.54 to 0.88$)$, 0.89 (95\% Cl: 0.86 to 0.91 ), and 0.91 ( $95 \%$ Cl: 0.88 to 0.92 ) at $0 \%, 10 \%$ and $12 \%$, respectively. Although, the reliability of the C-protocol with 12\% grade came close to that of the G-protocol, a linear contrast between the estimated ICC(1) of the C-protocol with 12\% grade and the ICC(1) of the G-protocol was statistically significant $(z=4.67, P<.001)$. After accounting for the difference in the $C$ - and $\mathrm{G}$-protocols and the influence of grade, some residual heterogeneity was still present $(\mathrm{Q}=$ $22.51, \mathrm{df}=12, \mathrm{P}=.03$ ). Table 3 summarizes these results. The ICC's for ICD and ACD for the C- and G-protocol of each study are graphically represented in forest plots. (Figure $2 a$ and $2 b$ ) In addition, leaving out the largest study of Labs, et al. ${ }^{44}$ from the analysis led to the same conclusions as reported above.

\section{Discussion}

The present results suggest that the reliability of treadmill assessments is highest when the ACD is measured in combination with the use of a G-protocol. When a C-protocol must be used for practical reasons (eg, due to the unavailability of a treadmill which is automatically adjustable in gradient), a C-protocol should be used with a $12 \%$ grade. The ACD should still be selected as the primary outcome measure in that case. Furthermore, we did not find a difference between three investigated G-protocols, which corresponds to the apparently equal workloads of these protocols (Figure $1 \mathrm{~b}$ ) 
Current guidelines

Treadmill testing is considered an objective method for assessing therapy success and for comparing the effectiveness of different treatments. However, European and North American guidelines from the past decades provide different recommendations regarding the use of treadmill testing in clinical trials. Heidrich et al. stated in the "Guidelines for therapeutic studies in Fontaine's stage II-IV peripheral arterial disease" from the German Society of Angiology that the primary test outcome should be the ICD assessed with a C-test (3 km/hour 12\%). ${ }^{50}$ The Vascular Clinical Trialists advises to use a G-test with a large dynamic range.

Table 2: Walking distances as measured during the first treadmill test and reported or calculated ICC(1) from included studies

\begin{tabular}{|c|c|c|c|c|c|c|c|c|c|}
\hline \multirow[t]{2}{*}{ Study } & \multicolumn{3}{|l|}{$\begin{array}{l}\text { Treadmill } \\
\text { protocol }\end{array}$} & \multicolumn{2}{|l|}{ ICD } & \multicolumn{2}{|l|}{$A C D$} & \multirow[t]{2}{*}{$\begin{array}{l}\mathrm{ICC}(1) \\
\mathrm{ICD}\end{array}$} & \multirow[t]{2}{*}{$\begin{array}{l}\mathrm{ICC}(1) \\
\mathrm{ACD}\end{array}$} \\
\hline & $\mathrm{C} / \mathrm{G}$ & $\begin{array}{l}\text { Velocity } \\
(\mathrm{km} / \mathrm{h})\end{array}$ & $\begin{array}{l}\text { Grade } \\
\%\end{array}$ & $\begin{array}{l}\text { Mean } \\
(\mathrm{m})\end{array}$ & SD & $\begin{array}{l}\text { Mean } \\
(\mathrm{m})\end{array}$ & SD & & \\
\hline Cachovan et & C & 3.0 & 12 & 87.8 & 60.3 & 149.2 & 120.0 & 0.880 & 0.910 \\
\hline al. 1999 & G1 & 3.0 & & 165.5 & 122.1 & 262.3 & 177.4 & 0.870 & 0.910 \\
\hline Degischer et & C & 2.0 & 12 & 136 & 61 & 250 & 109 & 0.792 & 0.843 \\
\hline al. 2002 & c & 3.2 & 0 & 187 & 121 & 405 & 219 & 0.817 & 0.614 \\
\hline & c & 3.2 & 12 & 102 & 46 & 204 & 116 & 0.713 & 0.855 \\
\hline & c & 4.0 & 0 & 160 & 75 & 315 & 114 & 0.828 & 0.835 \\
\hline & C & 4.0 & 12 & 102 & 37 & 167 & 80 & 0.833 & 0.952 \\
\hline Chaudhry et & C & 3.2 & 10 & & & & & 0.680 & 0.930 \\
\hline al. 1997 & G1 & 3.2 & & & & & & 0.840 & 0.980 \\
\hline Gardner et & C & 3.2 & 12 & 74 & 35 & 176 & 70 & 0.484 & 0.715 \\
\hline al. 1991 & G2 & 3.2 & & 179 & 82 & 376 & 168 & 0.600 & 0.883 \\
\hline $\begin{array}{l}\text { Gardner } \\
1997\end{array}$ & G2 & 3.2 & & & & $237^{*}$ & $178^{*}$ & & 0.950 \\
\hline $\begin{array}{l}\text { Labs et al. } \\
1999\end{array}$ & G2 & 3.2 & & 467.3 & 345 & 939 & 652.6 & 0.820 & 0.952 \\
\hline $\begin{array}{l}\text { Labs et al. } \\
1998\end{array}$ & c & 3.2 & 12 & 84.52 & 65.28 & 145.77 & 86.89 & 0.870 & 0.910 \\
\hline $\begin{array}{l}\text { Zwierska et } \\
\text { al. } 2004\end{array}$ & c & 3.2 & 12 & $75^{*}$ & $71^{*}$ & $115^{*}$ & $93^{*}$ & 0.880 & 0.870 \\
\hline
\end{tabular}

C/G indicates continuous protocol or graded protocol; $G 1$, graded protocol 1 with an increase in grade of $3.5 \%$ every 3 minutes to a maximum of 17.5\%; G2, graded protocol 2 with an increase in grade of $2 \%$ every 2 minutes to a maximum of $18 \%$; ICD, initial claudication distance; $A C D$, absolute claudication distance; ICC (1), intraclass correlation coefficient type 1.

* Mean of all measurements
Figure 2a: Forest plot of the ICC(1) values for ICD.

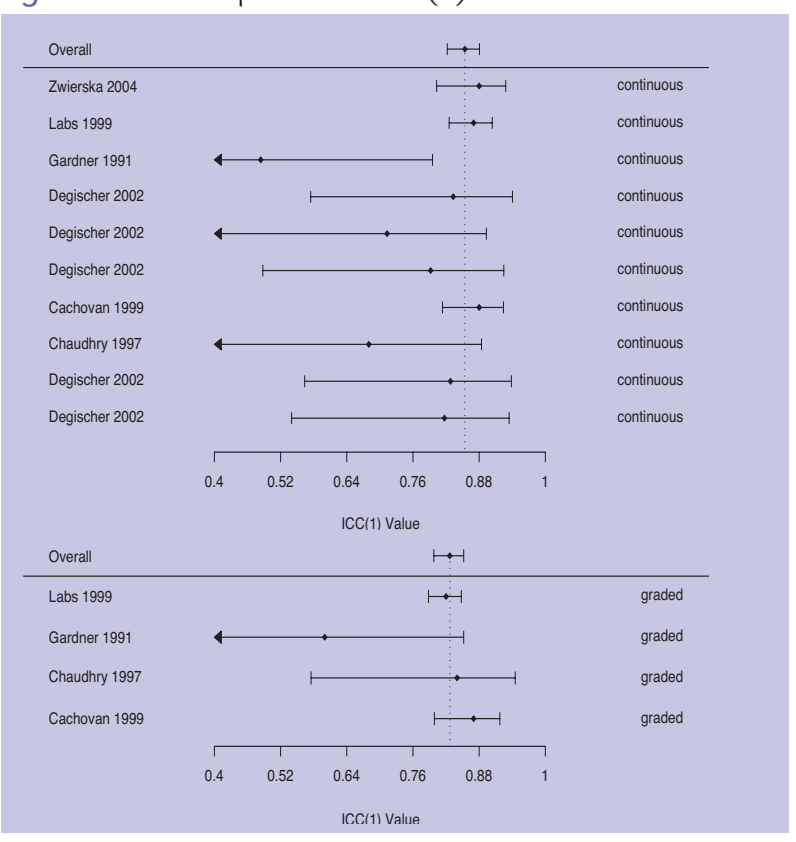

Figure $2 b$ : Forest plot of the ICC(1) values for $A C D$.

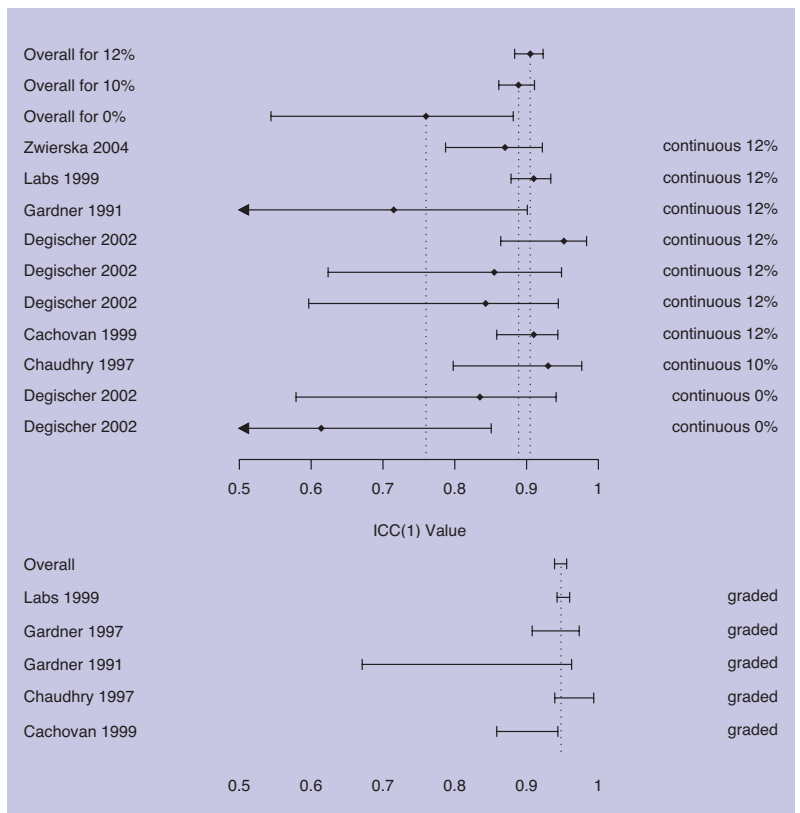

The preferable primary test outcome (ICD or ACD) is not described. Although it is not stated directly whether the ICD or ACD is the preferable outcome, it is described that the coefficient of variation of the ACD is estimated lower than the ICD (12\% versus 20\%), which implies that the ACD should be preferred. More recent clinical trial guidelines in PAD recommend measurement of the ACD by treadmill testing, which may be performed with a C-test (3.2 km/hour and 12\%) 


\begin{tabular}{|c|c|c|c|}
\hline & Continuous protocol & Graded protocol & $\begin{array}{l}\text { p-value for } \\
\text { test of the } \\
\text { difference }\end{array}$ \\
\hline \multirow{6}{*}{$\begin{array}{l}\text { ICD } \\
\text { ACD }\end{array}$} & Estimated ICC(1) (95\% Cl) & Estimated ICC(1)(95\% Cl) & \multirow{6}{*}{$.16<.001$} \\
\hline & 0.85 (0.82 to 0.88$)$ & 0.83 (0.80 to 0.85$)$ & \\
\hline & $0.90(0.88$ to 0.92$)$ & 0.95 (0.94 to 0.96) & \\
\hline & $0 \%$ grade $0.76(0.54$ to 0.88$)$ & & \\
\hline & $10 \%$ grade 0.89 (0.86 to 0.91$)$ & & \\
\hline & $12 \%$ grade 0.91 (0.88 to 0.92$)$ & & \\
\hline
\end{tabular}

ICC(1) denotes intraclass correlation coefficient type 1; ICD, initial claudication distance; $A C D$, absolute claudication distance.

or G-test (no advise), except for extreme walking distances when a G-test should be performed. ${ }^{51}$ The American College of Sports Medicine advises to perform exercise testing with a graded treadmill protocol (at $3.2 \mathrm{~km} /$ hour with 2\% increase every 2 minutes or 3.5\% increase every 3 minutes) or a gradual ramp protocol, starting at low speed and without indine and increasing continuously in speed and incline. The ICD and ACD should be recorded. ${ }^{52}$ Our results would suggest that in terms of reliability, the best measurement should be the ACD assessed by a G-protocol as a first choice and the ACD of the C-protocol as a second choice based on the estimated ICCS. Reliability for the ICD for as well the C- as the G-protoco is, although the ICCs are still reasonably high, lower than the estimated reliability for the ACD. From the patient's perspective, the increase in ICD after treatment could be of more concern than the increase of the ACD because it defines the range of activities that can be performed without complaints. However, most patients will continue to walk after appearance of the first signs of pain (ICD), few will walk until their maximum pain threshold (ACD) is reached during the course of daily activities.

Treadmill testing

Although treadmill testing is indeed the gold standard for the assessment of walking distances there are several limitations. In clinical trials there is an external validity problem since patients with PAD can be asymptomatic or have non-typical symptoms..$^{53}$ This might hamper the enrollment of patients with typical intermittent claudication symptoms. Furthermore, there can be a pronounced baseline variability in walking distance that can differ between days. It is recommended that patients who by history have a recognized potential (eg, recent phases of deterioration or improvement, or recent surgical or peripheral vascular intervention) for instability should be excluded. Testing for baseline stability in the run-in phase should not be mandatory. ${ }^{51}$ Moreover, in daily practice treadmill testing is impractical, it is time-consuming, requires adequate equipment and personnel, and it is not directly available for all physicians. A large discrepancy has been reported between walking ability and claudication walking distances as measured on a treadmill, ${ }^{2-4}$ implying that treadmill assessments may not be representative of daily life walking ability. Alternative, more simple tests include a 6 -minute walking test ${ }^{16,54}$, a shuttle walking-test ${ }^{16,45}$, a PADHOC device ${ }^{22}$, and a GPS device. ${ }^{55}$ Furthermore, a simple questionnaires like the Walking Impairment Questionnaire (WIQ) could be used in properly designed trials..$^{56,57} \mathrm{~A}$ recent study of McDermott et al. indicated that functional performance measures as the 6 -minute walking test correlate more closely to physical activity levels during daily life than treadmill walking performance. ${ }^{58}$
Methodological points of attention

An adequate literature search was performed and 8 studies, analyzing 15 treadmill protocols in a total of 658 patients, could be included. Although the study designs of nine other studies met the inclusion criteria for this analysis, they could not be included because of lack of ICC data. In these nine studies 12 treadmill protocols in 852 patients were analyzed. A description of these studies is presented in Table 4. Even though nine out of seventeen eligible studies were excluded, the majority of the excluded studies presented results consistent with this meta-analysis. Another limitation is that four of the included studies in this analysis involved a rather small number of patients.

Another subject of discussion is the type of ICC (absolute agreement or consistency) and the type of statistical model that is most sensible for this type of data. An ICC for absolute agreement would only be equal to 1 if the ICD/ACD values of the repeated measurements would be exactly identical for each person. A constant difference for all individuals between the repeated measurements reduces the value of the ICC below unity. On the other hand, an ICC for consistency would not be influenced by changes that affect all individuals equally Therefore, an increase in walking distance, for example due to a learning effect, would have no influence on the ICC for consistency, as long as this increase is consistent for all patients. Furthermore, there are various models that can be assumed to describe the structure of this type of data. Ideally, a fixed effect for time should be included in the model, since the measurement occasions are not interchangeable. Therefore, either an $\operatorname{ICC}(A, 1)$ or an $\operatorname{ICC}(C, 1)$ could be considered a more appropriate measure of the reliability, either in terms of absolute agreement or in terms of consistency. ${ }^{8}$ However, only the ICC(1), which is a measure of absolute agreement not considering a potential time effect, was reported or could be computed for all studies. If there are large changes in the mean ICD and ACD values over time, then the ICC $(1)$ will generally be smaller than the corresponding $\operatorname{ICC}(A, 1)$ value. Since the mean ICD and ACD could be considered to be stable over time due to the limited period of time between the tests, the difference between these two types of ICC should be relatively minor.

Finally, since some of the studies used multiple protocols with the same individuals, we had to compute the covariance between the resulting dependent ICC (1) values. To use the equation for the covariance given by Donner and Zou, ${ }^{10}$ we needed to know the correlation between the ICD (and ACD) values across protocols. None of the studies reported that information, so we estimated that value at 0.7 and conducted sensitivity analyses (using values between 0 and 0.9) to examine the robustness of the results to this assumed value. The results from the sensitivity analyses indicated that all of the conclusions are robust to misspecification of this correlation.

Future implications

The results of this study would suggest that many guidelines on clinical trials in patients with PAD require updating concerning walking ability assessment by treadmill testing. Ideally, there should be one international preferable standard. The present analysis suggests that a graded test protocol with absolute claudication distance as the primary outcome measure is the test modality with highest reliability. Estimated reliabilities for the C-protocol and the ICD were also reasonably high, indicating that under certain circumstances these could also be an adequate test protocol and outcome measurement. However, with regard to adequate comparison of future studies, preferably, guidelines for clinical trials on treatment of patients with peripheral arterial disease should be adapted and a uniform test protocol should be advised for future studies. 


\begin{tabular}{|c|c|c|c|c|c|}
\hline \multirow[t]{2}{*}{ study with year } & \multirow{2}{*}{$\begin{array}{l}\text { number of } \\
\text { patients }\end{array}$} & \multicolumn{3}{|c|}{ treadmill protocol } & \multirow[t]{2}{*}{ Main conclusion on treadmill testing } \\
\hline & & $\begin{array}{l}\text { Velocity } \\
(\mathrm{km} / \mathrm{h})\end{array}$ & $\begin{array}{l}\text { Grade } \\
(\%)\end{array}$ & $C / G$ & \\
\hline Brass et al 2007 & 386 & 3.2 & & G2 & $\begin{array}{l}\text { G-test quantifies ACD with high } \\
\text { reproducibility and stability over time }\end{array}$ \\
\hline Clyne et al 1979 & 117 & 2 & 0 & C & $\begin{array}{l}\text { The ACD achieved was reproducible. } \\
\text { Highly significant delay of the } \\
\text { ICD during the second test }\end{array}$ \\
\hline Cristol et al 1985 & 75 & 3 & 12 & C & $\begin{array}{l}\text { Overall reproducibility of the } \\
\text { ACD was } 10.8 \% \pm 8.6 \text { with } \\
\text { large individual variation }\end{array}$ \\
\hline Harris et al 1987 & 25 & 2.4 & 0 & C & $\begin{array}{l}\text { Significant increase in ICD and } \\
\text { ACD on test } 3 \text { compared with test } 1\end{array}$ \\
\hline Hiatt et al 1988 & 20 & $\begin{array}{l}3.2 \\
4.8\end{array}$ & & $\begin{array}{l}\text { G1 } \\
\text { G3 }\end{array}$ & $\begin{array}{l}\text { An accurate and reproducible } \\
\text { estimation of ACD could be achieved } \\
\text { from either treadmill protocol }\end{array}$ \\
\hline $\begin{array}{l}\text { Johnston et al } \\
1987\end{array}$ & 15 & 2.4 & 12 & C & The ACD proved to be reproducible \\
\hline Laing et al 1986 & 50 & 4 & 10 & C & $\begin{array}{l}\text { The ICD and ACD may vary } \\
\text { considerably from visit to visit }\end{array}$ \\
\hline $\begin{array}{l}\text { Lindgärde et al } \\
1996\end{array}$ & 154 & 3.2 & 12.3 & C & $\begin{array}{l}\text { Overall, the reproducibility of treadmill } \\
\text { results was high. Correlation } \\
\text { coefficients for ICD and ACD were } \\
>0.9 \text { (exact ICC data were not given) }\end{array}$ \\
\hline $\begin{array}{l}\text { Peräkylä et al } \\
1998\end{array}$ & 10 & $\begin{array}{l}3.2 \\
3.2 \\
3.2\end{array}$ & $\begin{array}{l}0 \\
12\end{array}$ & $\begin{array}{l}\mathrm{C} \\
\mathrm{C} \\
\mathrm{G} 2\end{array}$ & $\begin{array}{l}\text { Exercise testing appeared inaccurate. } \\
\text { The G-test was most reproducible } \\
\text { and the C-test with } 0 \% \text { incline least } \\
\text { reproducible. ACD seemed to be } \\
\text { more reliable than ICD }\end{array}$ \\
\hline
\end{tabular}

C/G indicates continuous protocol or graded protocol; $G 1$, graded protocol 1 with an increase in grade of 3.5\% every 3 minutes to a maximum of 17.5\%; G2, graded protocol 2 with an increase in grade of 2\% every 2 minutes to a maximum of 18\%; G3, graded protocol 3 with increase in grade of $2.5 \%$ every 3 minutes; ICD, initial claudication distance; $A C D$, absolute claudication distance; ICC, intraclass correlation coefficient

\section{References}

1. Muller-Buhl U, Kirchberger I, Wiesemann A.

Relevance of claudication pain distance in patients with peripheral arterial occlusive disease. VASA 1999;28:25-29.

2.Siggaard-Andersen J, Petersen FB. Intermitten claudication. A comparison between subjective and measured claudication walking distance. Angiology $1968 ; 19: 426-434$

3.Watson CJ, Phillips D, Hands L, Collin J. Claudication distance is poorly estimated and inappropriately measured. Br J Surg. 1997;84:1107-1109.

4. Gardner AW, Montgomery PS, Afaq A. Exercise performance in patients with peripheral arteria disease who have different types of exertional le pain. J Vasc Surg. 2007;46:79-86

5. Bruce RA, Kusumi F, Hosmer D. Maximal oxygen intake and nomographic assessment of functional aerobic impairment in cardiovascular disease. Am heart J. 1973;85:546-562.

6. Hiatt WR, Nawaz D, Regensteiner JG, Hossack KF. The evaluation of exercise performance in patients with peripheral vascular disease. J Cardiopulmonary Rehabil 1988:12:525-532.

7. Gardner AW, Skinner IS, Cantwell BW, Smith LK. Progressive vs single-stage treadmill tests for evaluation of claudication. Med Sci Sports Exerc 1991:23:402-408

8. McGraw KO, Wong SP. Forming inferences about some intraclass correlation coefficients. Psychol Methods. 1996;1:30-46.

9. Fisher RA. Statistical methods for research workers. Edinburgh: Oliver and Boyd 1925.

10. Donner A, Zou G. Testing the equality of dependent intraclass correlation coefficients. Statistician. 2002:51:367-379.

11. Chaudhry H. Exercise testing: which protocol? Critical Ischaemia.6(2):43-48.
12. Duprez D, de Backer T, de Buyzere M, Clement DL. Estimation of walking distance in intermittent claudication: need for standardization. Eur Heart J. 1999;20:641-644.

13. Kirby RL. Reliability of treadmill exercise test for patients with claudication. Can I Surgery. $1988 ; 31: 304$.

14. Froelicher VF, Jr., Brammell H, Davis $G$, Noguera I, Stewart A, Lancaster MC. A comparison of the reproducibility and physiologic response to three maximal treadmill exercise protocols. Chest. 1974;65:512-517.

15. Kirby RL, Marlow RW. Reliability of walking endurance with an incremental treadmill test. Angiology. 1987:38:524-529.

16. da Cunha-Filho IT, Pereira DA, de Carvalho AM, Campedeli L, Soares M, de Sousa Freitas $J$. The reliability of walking tests in people with claudication. Am J Phys Med Rehabil 2007;86:574-582.

17. Bader DS, Maguire TE, Balady GJ. Comparison of ramp versus step protocols for exercise testin in patients $>$ or $=60$ years of age. American . Cardiol. 1999;83:11-14.

18. Colt JD. A reproducible treadmill tes for intermittent claudication. Angiology. 1971;22:375-379

19. Rosfors S, Celsing F, Eriksson M. Transcutaneous oxygen pressure measurements in patients with intermittent claudication. Clin Physiol 1994:14:385-391.

20. Berglund B, Eklund B. Reproducibility of treadmil exercise in patients with intermittent claudication. Clin Physiol. 1981;1:253-256

21. Amirhamzeh MM, Chant HJ, Rees JL, Hands $\mathrm{L}$, Powell RJ, Campbell WB. A comparative study of treadmill tests and heel raising exercise for peripheral arterial disease. Eur J Vasc Endovasc Surg. 1997;13:301-305. 
22. Coughlin $P A$, Kent PJ, Turton EP, Byrne $P$, Berridge DC, Scott DJ, et al. A new device for the measurement of disease severity in patients with intermittent claudication. Eur J Vasc Endovasc Surg. 2001;22:516-522.

23. Gardner AW, Skinner JS, Vaughan NR, Bryant CX, Smith LK. Comparison of three progressive exercise protocols in peripheral vascular occlusive disease. Angiology. 1992;43:661-671.

24. Gardner AW, Skinner JS, Vaughan NR, Bryant CX, Smith LK. Comparison of treadmill walking and stair climbing over a range of exercise intensities in peripheral vascular occlusive disease. Angiology. 1993;44:353-360.

25. Gardner AW, Ricci MA, Case TD, Pilcher DB. Practical equations to predict claudication pain distances from a graded treadmill test. Vasc Med 1996; 1:91-96.

26. Leder U, Saul T, Frankenstein L, Krack A, Baer $\mathrm{H}$, Poehlmann $\mathrm{G}$, et al. Exercise capacity and Doppler pressure measurements in symptomatic peripheral arterial obstructive disease. VASA. 2002:31:107-110.

27. Riebe D, Patterson RB, Braun CM. Comparison of two progressive treadmill tests in patients with peripheral arterial disease. Vasc Med. 2001;6:215-221

28. Petersen FB. The effects of varying walking speeds when measuring the claudication distance on horizontal and sloping levels. Acta Chir Scand. 1967;133:627-630

29. Cachovan M. Methods and results of controlled walking training in patients with peripheral arteria occlusive disease. Z Artztl Fortbild Qualitatssich. 1997;93:626-632.

30. Cameron AE, Porter A, Rosser S, Da Silva AE, De Cossart LM. The Stresst'er ergometer as an alternative to treadmill testing in patients with claudication. Eur Vasc Endovasc Surg. 1997;14:433-438
31. Maass U, Alexander K, Schneider B, Torok M. [The reproducibility of treadmill-ergometric studies for the determination of the painless walking distance in patients with arterial occlusive diseases]. VASA. 1980;9:182-185.

32. Hillestad LK. The peripheral blood flow in intermitten claudication. IV. The significance of the claudication distance. Acta Med Scand. 1963; 173:467-478.

33. Ouriel K, McDonnell AE, Metz CE, Zarins CK. Critical evaluation of stress testing in the diagnosis of peripheral vascular disease. Surgery. 1982;91:686-693.

34. Clyne CA, Tripolitis A, Jamieson CW, Gustave $R$, Stuart F. The reproducibility of the treadmill walking test for claudication. Surg Gynecol Obstet. 1979;149:727-728.

35. Harris $K A$, Meads $G E$. A single treadmill exercise test does not accurately quantitate claudication. Can J Surg. 1987:30:446-448.

36. Laing S, Greenhalgh RM. Treadmill testing in the assessment of peripheral arterial disease. Int Angiol. 1986;5:249-252.

37. Lindgarde F, Labs KH, Rossner M. The pentoxifylline experience: exercise testing reconsidered. Vasc Med. 1996:1:145-154.

38. Perakyla T, Tikkanen H, von Knorring J, Lepantalo M. Poor reproducibility of exercise test in assessment of claudication. Clin Physiol. 1998;18:187-193.

39. Brass EP, Jiaob J, Hiatt W. Optimal assessment of baseline treadmill walking performance in claudication clinical trials. Vasc Med. 2007;12:97-103.

40. Johnston KW, Hosang MY, Andrews DF. Reproducibility of noninvasive vascular laboraton measurements of the peripheral circulation J Vasc Surg. 1987;6:147-151.

41. Cristol R, Graisely B. EEvaluation of intermittent claudication using a treadmill with measurement of systolic pressure at the ankle following exercise]. J Mal Vasc. 1985:10:101-107.
42. Gardner AW. Reliability of transcutaneous oximeter electrode heating power during (excise in patients with intermittent claudication. Angiology. 1997;48:229-235.

43. Labs K-H, Roessner M, Aschwanden M, Jeanneret Ch, Gehrig A, Jäger KA. Reproducibility of fixed load treadmill testing. J Vasc Invest. 1998:4:55-60.

44. Labs KH, Nehler MR, Roessner M, Jäger KA, Hiatt WR. Reliability of treadmill testing in peripheral arterial disease: a comparison of a constant load with a graded load treadmill protocol. Vasc Med. 1999;4:239-246.

45. Zwierska I, Nawaz S, Walker RD, Wood RF, Pockley RG, Saxton JM. Treadmill versus shuttle walk tests of walking ability in intermittent claudication Med Sci Sports Exerc. 2004:36:1835-1840.

46. Cachovan M, Rogatti W, Woltering F, Creutzig A, Diehm C, Heidrich $\mathrm{H}$, et al. Randomized reliability study evaluating constant-load and graded-exercise treadmill test for intermittent claudication. Angiology. 1999;50:193-200.

47. Degischer S, Labs KH, Aschwanden M, Tschoepl M, Jaeger KA. Reproducibility of constant-load treadmill testing with various treadmill protocols and predictability of treadmill test results in patients with intermittent claudication. J Vasc Surg. 2002;36:83-88.

48. Chaudhry H, Holland A, Dormandy J. Comparison of graded versus constant treadmill test protocols for quantifying intermittent claudication. Vasc Med. 1997;2:93-97.

49. Gardner AW, Skinner JS, Smith LK. Effects of handrail support on claudication and hemodynamic responses to single-stage and progressive treadmill protocols in peripheral vascular occlusive disease Am J Cardiol. 1991;68:99-105.

50. Heidrich H, Cachovan M, Creutzig A, Rieger H, Trampisch HJ. Guidelines for therapeutic studies in Fontaine's stages II-IV peripheral arterial occlusive disease. German Society of Angiology. VASA. 1995;24:107-119.
51. Labs KH, Dormandy JA, Jaeger KA, Stuerzebecher CS, Hiatt WR. Transatlantic Conference on Clinica Trial Guidelines in Peripheral Arterial Disease: clinical trial methodology. Basel PAD Clinical Trial Methodology Group. Circulation. 1999;100:e75-81.

52. ACSM's guidelines for exercise testing and prescription / American College of Sports Medicine. Baltimore: Lippincott Williams and Wilkins 2006.

53. Hiatt WR, Hoag S, Hamman RF. Effect of diagnostic criteria on the prevalence of periphera arterial disease. The San Luis Valley Diabetes Study. Circulation. 1995:91:1472-1479.

54. Montgomery PS, Gardner AW. The clinical utility of a six-minute walk test in peripheral arterial occlusive disease patients. J Am Geriatr Soc 998:46:706-711.

55. Le Faucheur $A$, Abraham $P$, Jaquinandi V, Bouye P, Saumet JL, Noury-Desvaux B. Measurement of walking distance and speed in patients with peripheral arterial disease: a novel method using a global positioning system. Circulation. 2008;117:897-904

56. MCDermott MM, Liu K, Guralnik JM, Martin $\mathrm{GJ}$, Criqui MH, Greenland P. Measurement of walking endurance and walking velocity with questionnaire: validation of the walking impairment questionnaire in men and wome with peripheral arterial disease. J Vasc Surg. 1998;28:1072-1081.

57.Nicolaï SP, Kruidenier LM, Rouwet EV, Graffius K, Prins MH, Teijink JA. The Walking Impairment Questionnaire: an effective tool to assess the effect of treatment in patients with intermittent claudication J Vasc Surg. 2009;in press.

58. McDermott MM, Ades PA, Dyer A, Guralnik JM, Kibbe M, Criqui MH. Corridor-based functiona performance measures correlate better with physical activity during daily life than treadmil measures in persons with peripheral arterial disease. J Vasc Surg. 2008:48:1231-1237, 1237 e 1231. 


\section{CHAPTER 5}

\section{Extending the range of treadmill testing for patients with peripheral arterial disease}




\section{Abstract}

Purpose: There is a need to evaluate patients with peripheral arterial disease (PAD) with a limited or extended walking distance. We aimed to enable an estimation of walking distance as measured on a frequently used 'standard' graded $(3.2 \mathrm{~km} / \mathrm{h}, 2 \%$ increase per 2 minutes) protocol, for walking distances measured on protocols with a lower or higher workload.

Methods: Patients with PAD and an absolute claudication distance (ACD) of $<500$ or between 1000 and 1600 meters as measured with the 'standard' protocol were included. Four graded study treadmill protocols, 2 with lower and 2 with higher workload than the 'standard' protocol were developed. Two study protocols (low or high) and the 'standard' protocol were repeated in random order. Quality was determined with the intraclass correlation coefficient (ICC) and the coefficient of variation (COV). Orthogonal regression analysis was used to predict walking distances on the standard protocol based on the study protocols.

Results: Forty three patients with an $A C D<500$ meters and 23 patients with an $A C D$ between 1000 and 1600 meters were included. Since feasibility from the protocols with $2.0 \mathrm{~km} / \mathrm{h}$ and $2 \%$ increase every 2 minutes and $4.4 \mathrm{~km} / \mathrm{h}$ and $2 \%$ increase every minute was highest, they were calibrated against the 'standard' protocol and reliability was comparable with the 'standard' protocol. The COVs between the prediction of walking distance on the 'standard' protocol based on the new protocols and the measured distances were in the same range (22-25\%) as the variation measured performing the same treadmill test twice.

Conclusion: An accurate estimate of walking distance as measured on a 'standard' treadmill protocol can be derived from a protocol with a lower or higher workload.

\section{Introduction}

The accepted method to evaluate walking capacity and to assess the functional effect of treatment in patients with peripheral arterial disease (PAD) is treadmill testing. Unfortunately, clinical trials on patients with PAD use different treadmill protocols. Preferably, one standardized test protocol should be used to enable comparison of test results within and between patients and also across studies that evaluate treatments for patients with PAD. A recent meta-analysis suggested that the use of a graded test protocol with the absolute claudication distance (ACD) as the outcome measurement, is optimal. ${ }^{2}$

The most frequently applied graded tests uses a constant speed of $3.2 \mathrm{~km} / \mathrm{h}$ and, starting at $0 \%$, a $2 \%$ increase of incline every two minutes ${ }^{3}$ or $3.5 \%$ every three minutes. ${ }^{4}$ Unfortunately, these measurement protocols cannot be used for many PAD patients because of their limited maximal walking speed without using handrail support. ${ }^{5}$ Earlier reported results showed that approximately 15\% of the patients with PAD were initially not able to walk on this 'standard' graded protocol (3.2 $\mathrm{km} / \mathrm{h}$ and $2 \%$ increase every two minutes) while after adequate therapy this would be possible. ${ }^{6}$ This hampers adequate evaluation of the effect of therapy for the individual patient and within clinical trials. Furthermore, when the increase of ACD is evaluated in patients who are initially capable to walk the 'standard' protocol, for some patients the ACD after treatment becomes too long to be practical. In our experience approximately $35 \%$ of patients walk longer than 30 minutes on the 'standard' protocol after 6 months of supervised exercise therapy ${ }^{7}$ Hence, improvements or nuances in deterioration in walking distances can not be assessed.

Therefore, we developed 4 graded treadmill protocols, 2 with a lower and 2 with a higher workload than the 'standard' protocol. The goal of the present study was to evaluate whether these protocols are feasible for patients with severe symptoms (protocol with lower workload) or very mild symptoms (protocol with higher workload) of PAD. We also wanted to establish the relationship between the measurements from the new protocols and from the 'standard' protocol to be able to estimate the walking distance for the 'standard' protocol from walking distances measured on protocols with a higher or lower workload by means of a simple equation which can be used in clinical practice.

\section{Methods}

Patients with PAD, who were referred for community-based supenvised exercise therapy ${ }^{8}$ after confirmation of the diagnosis with an ankle brachial index (ABI) measurement below 0.9 at rest or an $\mathrm{ABI}$ decrease of more than 0.15 after exercise, were selected. Patients were eligible if they followed at least 3 months of supervised exercise therapy and if the increase in their walking distance had leveled off to no or minimal increase per week (plateau phase of therapy). Hence, the increase in effect of therapy within the two weeks study period was minimized. A 'standard' treadmill test (constant speed of $3.2 \mathrm{~km} / \mathrm{h}$ and $2 \%$ increase every 2 minutes was performed. ${ }^{3}$ To develop a protocol with a lower workload patients with an absolute claudication distance (ACD) of $<500$ meters were included and for the development of a protocol with a higher workload patients with an ACD between 1000 and 1600 meters were included. Patients presenting other reasons than intermittent claudication that caused them to stop walking during the first treadmill test were excluded. Written informed consent was obtained from all patients and the study was approved by the Institutional Review Board of the Atrium medical center Parkstad. 
Treadmill protocols

Within 2 weeks after the initial treadmill test that confirmed eligibility, all patients performed 5 treadmill tests with 3 different protocols in random order based on a computer generated randomization list created by an independent researcher. The initial test for all patients was the 'standard' graded protocol: constant speed of 3.2 $\mathrm{km} / \mathrm{h}$ and $2 \%$ increase every 2 minutes. Then, patients with an ACD of $<500$ meters performed in random order treadmill tests with the following treadmill protocols:

- Low 1.6: constant speed of $1.6 \mathrm{~km} / \mathrm{h}$ and $2 \%$ increase every 4 minutes - Low 2.0: constant speed of $2.0 \mathrm{~km} / \mathrm{h}$ and $2 \%$ increase every 4 minutes For patients with an ACD between 1000 and 1600 meters the following two protocols were used:

- High 4.4: constant speed of $4.4 \mathrm{~km} / \mathrm{h}$ and $2 \%$ increase every minute - High 4.8: constant speed of $4.8 \mathrm{~km} / \mathrm{h}$ and $2 \%$ increase every minute Treadmill tests were performed on separate days but within two weeks. All tests were performed twice, resulting in a total of six treadmill tests (the initial treadmill test to confirm eligibility with 5 extra tests). For practical reasons, the incline and duration of all test were maximized to $10 \%$ and 30 minutes, respectively. Patients were not allowed to hold the handrail. ${ }^{5}$ Furthermore, patients were blinded for speed, incline and duration of the test. The primary outcome measure was the ACD, defined as the distance at which severe claudication pain forced cessation of walking. The secondary outcome measure was the functional claudication distance (FCD), defined as the distance at which the patient preferred to stop because of claudication pain. ${ }^{9}$ ACD and FCD are to some extent subjective, relying on patient understanding of instructions and verbal reporting. For this reason, patients were instructed to indicate at which moment during the test they (a) would stop walking because of pain in daily life situation (FCD), (b) cannot walk on anymore (ACD) before every treadmill test. Furthermore, patients were asked about the tolerability of the new developed protocols.

\section{Statistical analysis}

The ACD and the FCD were expressed as distance in meters. First quality and tolerability of the new protocols was investigated to define the superior ones. Three methods were used to assess quality of the new developed protocols; reliability was assessed by means of an intraclass correlation coefficient (ICC), variability by means of the coefficient of variation (COV), and reproducibility was visualised in Bland-Altman plots. For the COV we calculated the average of and the difference between the two treadmill assessments for each patient. The COV was calculated as the standard deviation of the differences between the two treadmill assessments divided by the mean of the averages. Furthermore, a Bland-Altman plot is used to visualize the agreement between two clinical measurements. ${ }^{10}$ On the horizontal axis the mean of both measurements is presented and on the vertical axis the difference between the two measurements is presented. Tolerability was investigated by questionnaire.

With this study we aimed to enable an estimation of the walking distances for the 'standard' protocol from walking distances measured on protocols with a higher or lower workload. Since all measurements were performed twice, the mean of both measurements was used for analysis. Walking distances measured with the new protocols needed to be calibrated against walking distances measured with the 'standard' protocol. We used an orthogonal regression calibration model since as well the initial standard test as the new developed test include random measurement error. ${ }^{11}$ A usual linear regression analysis minimizes the square of the vertical distances of the data to the fitted regression line. In this case it should be inappropriate to define the measurements of the initial 'standard' test as a reference with less error than the new developed tests. In orthogonal regression analysis, as used in this study, the square of the perpendicular distances from the fitted line are used instead. The orthogonal regression coefficient is calculated as followed:

\section{$b=S y y-n S x x V\left((s y y-n S x x)^{2}+4 n(S x y)^{2}\right) / 2 S x y$.}

The $n$ is the ratio of measurement error variance of the two tests. Then the equation to predict walking distances is $a=\bar{y}-b \bar{x}$. Finally, we assessed quality of the calculated prediction equations. Therefore, we predicted the results on the 'standard' protocol based on the results from the lower and higher protocols. First, the COV between the actual measured results and the predicted results was calculated and then they were plotted in a Bland-Altman plot. In the ideal situation the difference between both measurements is zero, which is visualised for the individual measurements in the Bland-Altman plot Statistical analysis was performed with Excel 2000 for Windows and the SPSS statistical software package for Windows (Microsoft, Redmond, Wash), version 15.0 (SPSS Inc, Chicago, III).

\section{Results}

In total 66 patients were included, 43 patients with an $A C D<500$ meters and 23 patients with an ACD between 1000 and 1600 meters. Mean age was 66.8 years and $62.1 \%$ was male. The median ankle brachial index (ABI) was 0.70 and the mean body mass index (BMI) was 270. The group of patients with the shorter ACD was somewhat older and heavier, and had slightly lower ABI values. Baseline characteristics of the total group and after stratification for ACD are presented in Table 1. Four newly developed graded treadmill protocols and a frequently used 'standard' treadmill protocol were evaluated (Table 2).

\begin{tabular}{|c|c|c|c|}
\hline & $\begin{array}{l}\mathrm{ACD}<500 \text { meters } \\
\mathrm{N}=43\end{array}$ & $\begin{array}{l}\text { ACD between } 1000 \\
\text { and } 1600 \text { meter } \mathrm{N}=23\end{array}$ & $\begin{array}{l}\text { Total } \\
\mathrm{N}=66\end{array}$ \\
\hline Age - mean (SD) & $68.0(7.6)$ & 64.7 (9.5) & $66.8(8.4)$ \\
\hline Men - \% & 55.8 & 73.9 & 62.1 \\
\hline $\begin{array}{l}\text { Body Mass Index - } \\
\text { mean (SD) }\end{array}$ & $27.4(5.5)$ & 26.3 (3.9) & $27.0(4.9)$ \\
\hline $\begin{array}{l}\text { Ankle brachial index - } \\
\text { median (IQR) }\end{array}$ & $0.68(0.58-0.96)$ & $0.72(0.62-0.84)$ & $0.70(0.60-0.90)$ \\
\hline Hypertension - \% & 47.5 & 56.5 & 50.8 \\
\hline Diabetes mellitus - \% & 22.5 & 21.7 & 22.2 \\
\hline \multicolumn{4}{|l|}{ Smoking } \\
\hline Current smoker - \% & 52.5 & 43.5 & 49.2 \\
\hline Ex-smoker - \% & 37.5 & 47.8 & 41.3 \\
\hline Never smoked - \% & 10 & 8.7 & 9.5 \\
\hline
\end{tabular}

$A C D$ indicates absolute claudication distance, SD standard deviation, and IQR interquartile range 


\begin{tabular}{|c|c|c|c|c|c|c|}
\hline & $\begin{array}{l}\text { Speed } \\
\mathrm{km} / \\
\text { hour }\end{array}$ & Incline & $\begin{array}{l}\text { Distance } \\
\text { after } \\
30 \text { minutes } \\
\text { (meters) }\end{array}$ & $\begin{array}{l}\text { ICC* FCD } \\
95 \% \mathrm{Cl})\end{array}$ & $\begin{array}{l}\text { ICC*ACD } \\
(95 \% \mathrm{Cl})\end{array}$ & COV \\
\hline Low 1.6 & 1.6 & $\begin{array}{l}\text { Every } 4 \text { minutes } \\
2 \% \text { increase }\end{array}$ & 800 & $\begin{array}{l}0.88 \\
(0.79-0.94)\end{array}$ & $\begin{array}{l}0.84 \\
(0.71-0.91)\end{array}$ & $24.5 \%$ \\
\hline Low 2.0 & 2.0 & $\begin{array}{l}\text { Every } 4 \text { minutes } \\
2 \% \text { increase }\end{array}$ & 1000 & $\begin{array}{l}0.93 \\
(0.89-0.96)\end{array}$ & $\begin{array}{l}0.89 \\
(0.81-0.94)\end{array}$ & $22.4 \%$ \\
\hline Standard & 3.2 & $\begin{array}{l}\text { Every } 2 \text { minutes } \\
2 \% \text { increase }\end{array}$ & 1600 & $\begin{array}{l}0.87 \\
(0.79-0.92)\end{array}$ & $\begin{array}{l}0.95 \\
(0.92-0.97)\end{array}$ & \\
\hline High 4.4 & 4.4 & $\begin{array}{l}\text { Every minute } \\
2 \% \text { increase }\end{array}$ & 2200 & $\begin{array}{l}0.71 \\
(0.42-0.87)\end{array}$ & $\begin{array}{l}0.94 \\
(0.87-0.98)\end{array}$ & $19.4 \%$ \\
\hline High 4.8 & 4.8 & $\begin{array}{l}\text { Every minute } \\
2 \% \text { increase }\end{array}$ & 2400 & $\begin{array}{l}0.94 \\
(0.86-0.94)\end{array}$ & $\begin{array}{l}0.99 \\
(0.97-0.99)\end{array}$ & $9.0 \%$ \\
\hline
\end{tabular}

ICC indicates intra class coefficient, $A C D$ absolute claudication distance, $\mathrm{Cl}$ confidence interval, $\mathrm{COV}$ coefficient of variation calculated as the standard deviation of the differences divided by the average of the means.

*ICC of absolute agreement with a fixed effect for time was used (ICC $(A, 1)){ }^{16}$

Quality and tolerability of treadmill protocols

Quality assessment for the 'standard' protocol and the 4 new protocols consisted of

calculation of the ICC and COV (Table 2) and is presented as Bland-Altman plots (Figure 1). For the 'standard' test ( $n=66)$ the ICC for the ACD was 0.95 (95\%-Cl: $0.92-0.97)$. The

COVs for the ACD $<500$ meters and the ACD between 1000 and 1600 meters were 16.0\% and $18.5 \%$, respectively.

For the 'Low 2.0' protocol $(n=43)$ the ICC for the ACD was $0.89(95 \% \mathrm{Cl} 0.81-0.94)$ and the COV was $22.4 \%$. For the 'Low 1.6 ' protocol we found an ICC for the ACD of 0.84 (95\% Cl: $0.71-0.91$ ) and a COV of $24.5 \%$. Twelve out of 43 patients experienced the 'Low 1.6 ' protocol as inconvenient, since the slow speed induced balance problems during walking. For the 'Low 2.0' protocol none of the patients reported difficulties to cope with the requirements of this treadmill protocol.

The ICC for the ACD of the 'High 4.4' protocol ( $\mathrm{n}=23$ ) was 0.94 (95\% Cl: $0.87-0.98)$. For the 'High 4.8' protocol the ICC for the ACD was 0.99 ( $95 \%$ Cl: $0.97-0.99$ ). The COVs for the 'High 4.4' and 'High 4.8' were $19.4 \%$ and $9.0 \%$, respectively. In five out of 23 patients the 'High 4.4' protocol initiated cardiopulmonary limitations and 1 of these patients actually ended the test for this reason. The 'High 4.8' protocol initiated these problems in 7 patients and for 5 of them a cardiopulmonary factor was the reason for test cessation.

Based on the tolerability analysis, we decided to further only evaluate one protocol with lower workload ('Low 2.0') and one protocol with a higher workload ('High 4.4'). Reliability was visualized with Bland-Altman plots. In Figure 1, the difference between both treadmill measurements is presented against the mean of the two measurements. For the 'standard' protocol the variation of shorter walking distances $(n=43)$ between both measurements is smaller than for the longer walking distances $(n=23)$.
Relationship between treadmill protocols

In Figure 2 the individual test results of the 'Low 2.0' and the 'High 4.4' protocols are plotted against the 'standard' protocol. The equation to predict the walking distance of a patient on the 'standard' protocol based on results of the 'Low 2.0' protocol is walking distance standard protocol $=149.5+(0.38 *$ walking distance Low 2.0 protocol $)$. The COV between the predicted ACD based on the 'Low 2.0' protocol and the measured ACD on the 'standard

Figure 1: Bland-Altman plot visualising reliability of the 'standard' protocol, the 'Low 2.0' protocol, and the 'High 4.4' protocol.

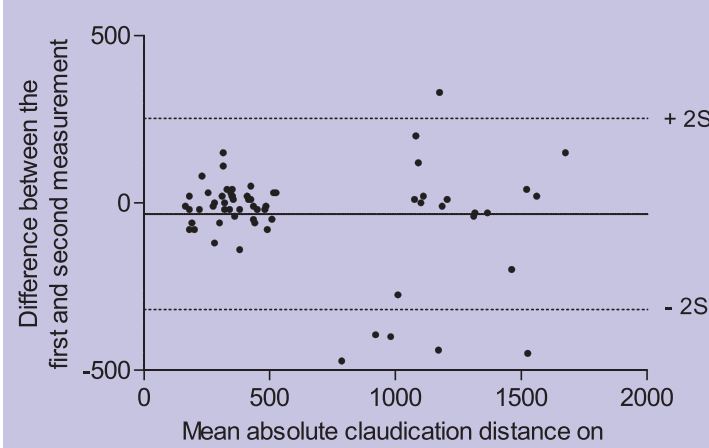

claudication distance on

the 'standard' protocol

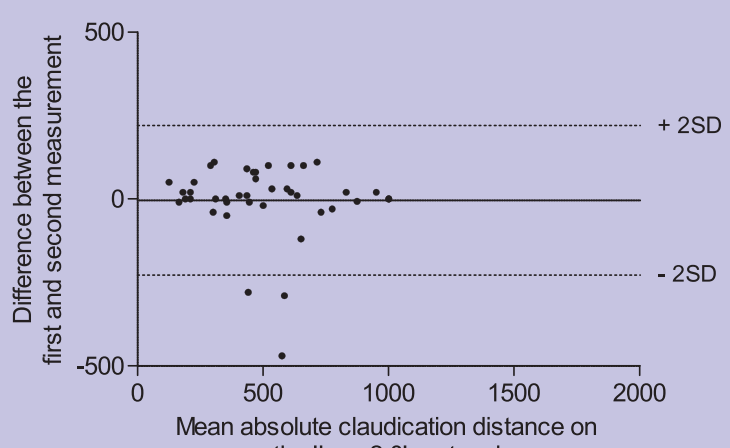

the 'Low 2.0' protocol

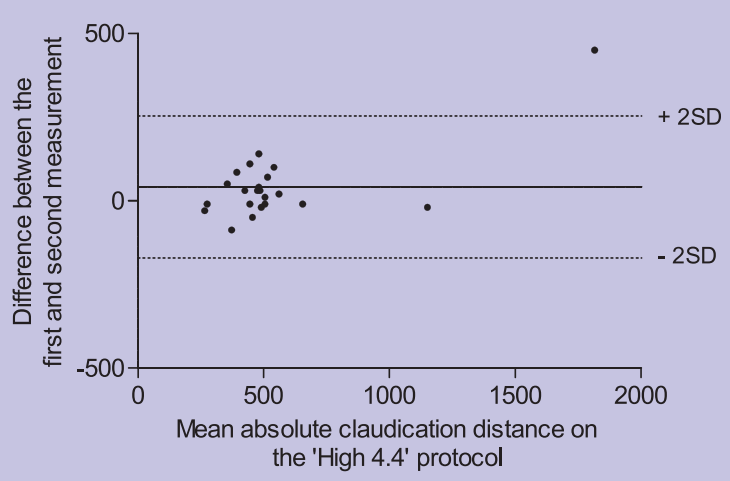


protocol was $25.0 \%$. The equation for the prediction of the 'standard' protocol based on the 'High 4.4' protocol is walking distance standard protocol $=695.1+(0.93 *$ walking distance High 4.4 protocol) with a COV of $22.0 \%$ between the predicted ACD on the 'standard' protocol and the measured ACD. The quality of both predictions is presented as Bland-Altman plots in Figure 3.

\section{Discussion}

The aim of our study was to enable an estimation of the walking distance for the 'standard' protocol from walking distances measured on protocols with a higher or lower workload. For this reason we developed and tested two study protocols with a lower workload ('Low 1.6' and 'Low 2.0') and two study protocols with a higher workload ('High 4.4' and 'High 4.8') than the 'standard' protocol. The tolerability of the 'Low 1.6 ' and 'High 4.8' protocols was low. Therefore, it was decided to calibrate the 'Low 2.0' and 'High 4.4' protocol against the 'standard' protocol. The prediction equations to predict walking distance intended for clinical use based on the 'Low 2.0' protocol and the 'High 4.4' protocol are:

- Predicted walking distance standard protocol $=149.5+(0.38 *$ walking distance Low 2.0 protocol $)$ - Predicted walking distance standard protocol $=695.1+(0.93 *$ walking distance High 4.4 protocol $)$ Quality of the new protocols, expressed as ICC and COV, was similar to the quality of the 'standard' protocol. The COVs based on the predicted walking distances based on the new protocols and the measured walking distances on the 'standard' protocol were acceptable with values ranging from 22.0 to $25.0 \%$. Because these COVs are not much larger than the

Figure 2: Scatter plots comparing the results of the 'standard' protocol with the 'Low 2.0' protocol and the 'High 4.4' protocol with the orthogonal regression lines

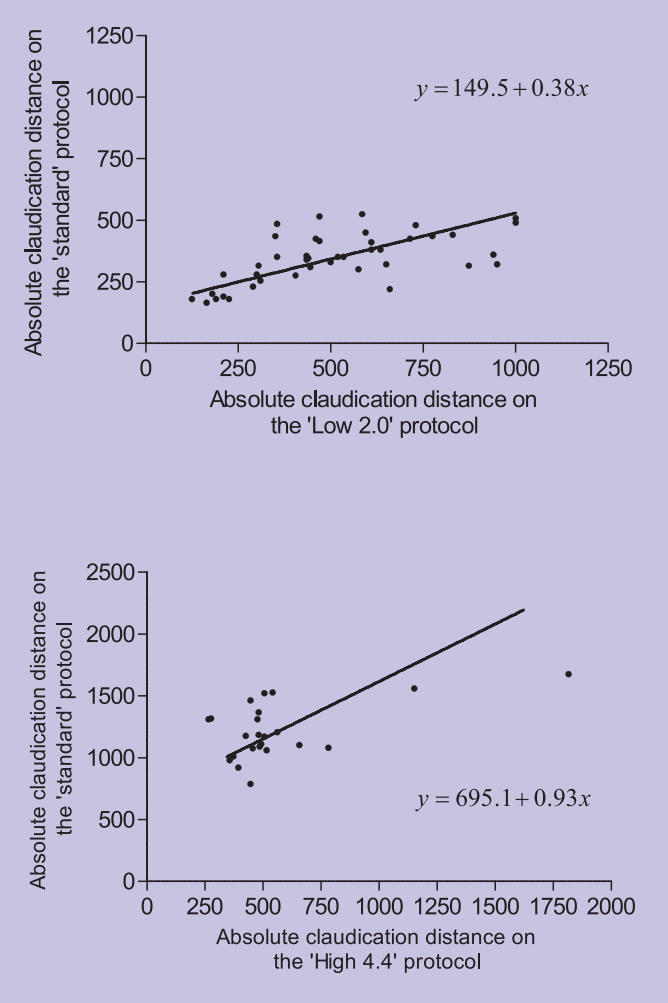

other COVs, the translation of the ACDs from the new protocols to the standard protocol seems to be adequate for clinical use. With the development of these new protocols the range of treadmill testing is extended from maximal 1600 meters to maximal 2740 meters (30 minutes on the 'High 4.4' protocol translates into 2740 meters on the 'standard' protocol). Evaluating supenised exercise therapy for intermittent claudication the new protocols are applicable for approximately $15 \%$ of patients who are initially not capable of performing a standard treadmill test and for approximately 35\% of patients, in whom a 'standard' treadmill test does not initiate intermittent claudication symptoms anymore after supenvised exercise therapy. In line with this, the new protocols are also expected to be useful to evaluate other therapies for intermittent claudication, e.g. the evaluation of peripheral vascular interventions.

Figure 3: Bland-Altman plot visualising quality of the predictions based on orthogonal regression

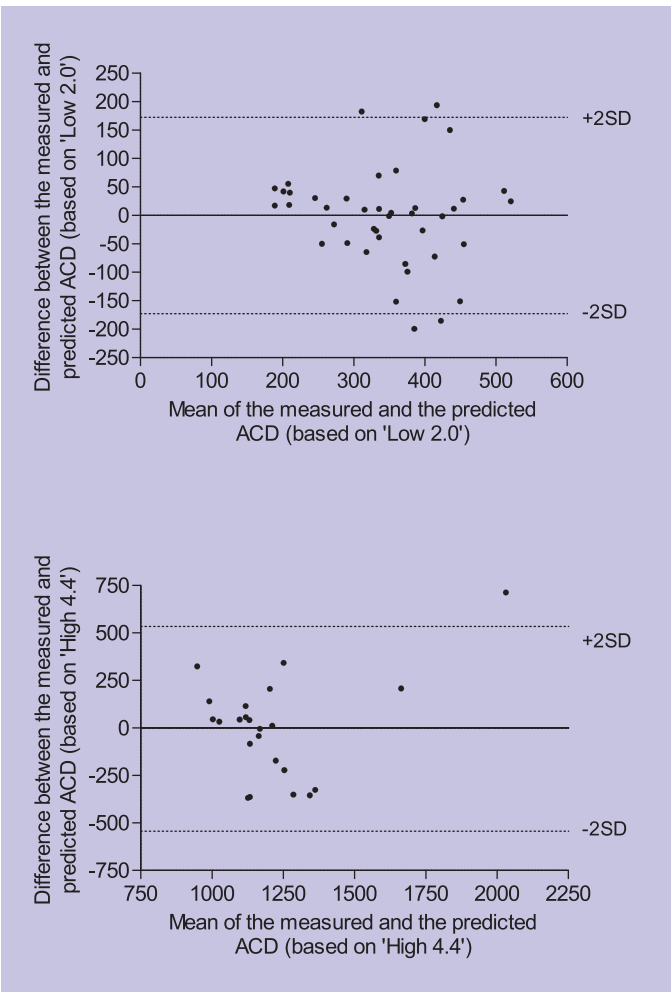

Several studies have been published regarding the comparison of treadmill protocols. ${ }^{12,13}$ Riebe et al developed a graded protocol with stages of 5 minutes starting at a lower speed (1.6 $\mathrm{km} / \mathrm{h}$ ) mainly for deconditioned patients. ${ }^{13}$ Walking distances were directly compared for 11 patients. No statistically significant differences in walking distances as measured with the new and the 'standard' protocol were found. In another study, the reproducibility and comparability of 5 constant loaded test protocols showed an adequate mathematical prediction based on metabolic equivalent (MET) normalization, which is only possible for constant loaded treadmil tests. ${ }^{12}$ However, these two studies do not allow translation of the measured walking distance on a slower protocol to a walking distance on the 'standard' protocol for evaluation of treatment Also, comparison for patients with a walking distance measurable on the 'standard' protoco at the beginning of treatment, with a larger increase in walking distance making a higher 
workload protocol necessary, is not possible. Based on the idea of MET normalization, ${ }^{12}$ we calculated the estimated energy expenditure, expressed as kilocalories, which is related to the MET equivalent of each stage of the graded treadmill tests. 1,14 However, this did not improve the fit of the data around the regression line when compared to using walking distances (data not shown).

An orthogonal regression equation was used to calibrate the new protocols against the 'standard' protocol. Quality of these predictions are visualised in Bland-Altman plots (Figure 3) and show a moderate translation with a wide variation. It is estimated that a patient walking 300 meters at the 'Low 2.0' protocol, walks 263.5 meters at the 'standard' protocol. For the 'High 4.4' protocol a walking distance of 1500 meters is translated to 2090.1 meters on the 'standard' protocol. Considering the observed interindividual differences (Figure 3) when walking on the new protocols and the 'standard' protocol, other factors than the ischemic component seem to influence walking distances on different protocols. This is in line with the finding that no relation was found between the angiographic extent of the disease in patients with PAD and the maximal walking distance.15 During exercise the lesions in the arterial supply of the leg muscles limit the necessary increase in blood flow. For example, differences between the available oxygen capacity at the start of the exercise, the oxygen supply during, and the effort necessary for the different treadmill protocols could result in variation between and within patients.

Limitations of the study

The major limitation of this study is that the 'Low' protocols were only tested in patients with a maximal walking distance on the standard protocol of $<500$ meters and, similarly for the 'High' protocols, only patients who walked between 1000 and 1600 meters on the 'standard' protocol were tested. For the low protocol extrapolation of the prediction equation to walking distances below about 200 meters does not seem to be possible because it would lead to a longer walking distance on the higher workload protocol. For the high workload protocol it is assumed that the calculated equation can be extrapolated to longer walking distances. Probably this assumption cannot be tested because of other reasons for test cessation than PAD. Another limitation is that the six treadmill tests were performed on different days within two weeks. Walking distances may vary between days and, although, we only included patients in the plateau phase of the supenvised exercise therapy, minor therapy effects can not be excluded. However, the random assignment of protocols may decrease this effect.

In conclusion, this study enables relevant estimations of walking distance as measured on a 'standard' treadmill protocol from a protocol with a lower workload, which might be necessary for patients with a very limited walking distance at the start of the therapy. Also, for patients with an increased walking distance after therapy it is possible to measure this distance on a higher protocol and translate this to the 'standard' protocol. Although, the coefficients of variation show a substantial amount of variation between the measured and estimated walking distances, this variation is acceptable since the COV values lie in the same range as those measured for performing the same treadmill test twice. During the evaluation of supervised exercise therapy in patients with intermittent claudication the new protocols are applicable for a substantial amount of patients.

\section{Acknowledgments}

We would like to thank Jeroen Voois, Marjolein Leerink, and Ivette Essers for performing the treadmill tests.

\section{References}

1. ACSM's guidelines for exercise testing and prescription / American College of Sports Medicine. Baltimore: Lippincott Williams and Wilkins 2006. 225-227 and 288-289

2. Nicolai SP, Viechtbauer W, Kruidenier LM, Candel MJ, Prins MH, Teiijink JA. Reliability of treadmill testing in peripheral arterial disease; a metaregression analysis. J Vasc Surg. 2009;50:322-9.

3. Gardner AW, Skinner JS, Cantwell BW, Smith LK. Progressive vs single-stage treadmill tests for evaluation of claudication. Med Sci Sports Exerc 1991;23:402-408.

4. Hiatt WR, Nawaz D, Regensteiner JG, Hossack KF. The evaluation of exercise performance in patients with peripheral vascular disease. $\downarrow$ Cardiopulmonary Rehabil. 1988;12:525-532.

5. Gardner AW, Skinner JS, Smith LK. Effects of handrail support on claudication and hemodynamic responses to single-stage and progressive treadmill protocols in peripheral vascular occlusive disease. Am J Cardio. 1991:68:99-105.

6. Kruidenier LM, Nicolaï SP, Hendriks EJ, Bollen EC, Prins MH, Teijink JA. Supervised exercise therapy for intermittent claudication in daily practice. J Vasc Surg 2009;49:363-70

7. Electronic Patient File Intermittent Claudication Web site [Internet]. Maastricht (The Netherlands): Center for Evidence Based Physiotherapy; [cited 2009 August 18].

Available from: https://claudicatio.cebp.nt/

8. Willigendael EM, Bendermacher BL, van der Berg C, Welten RJ, Prins MH, de Bie RA, et al. The development and implementation of a regional network of physiotherapists for exercise therapy in patients with peripheral arterial disease, a preliminary report. BMC Health Serv Res. 2005;5:49
9. Kruidenier LM, Nicolaï SP, Willigendael EM, de Bie RA, Prins MH, Teijink JA. Functional claudication distance: a reliable and valid measurement to assess functional limitation in patients with intermittent claudication. BMC Cardiovasc Disord. 2009;9:9

10. Bland JM, Altman DG. Statistical methods for assessing agreement between two methods of clinical measurement Lancet. 1986:1·307-310.

11. Carroll RJ, Ruppert D. The use and misuse of orthogonal regression estimation in linear errorsin-variables models. Am Statist. 1996;50:1-6.

12. Degischer S, Labs KH, Aschwanden M, Tschoepl M, Jaeger KA. Reproducibility of constant-load treadmill testing with various treadmill protocols and predictability of treadmill test results in patients with intermittent claudication. J Vasc Surg. 2002;36:83-88

13. Riebe D, Patterson RB, Braun CM. Comparison of two progressive treadmill tests in patients with peripheral arterial disease. Vasc Med. 2001:6:215-221.

14. Ainsworth BE, Haskell WL, Leon AS, Jacobs DR $\mathrm{Jr}$, Montoye HJ, Sallis JF, et al. Compendium of physical activities: classification of energy costs of human physical activities. Med Sci Sports Exerc. 1993;25:71-80.

15. Muller-Buhl U, Kirchberger I, Wiesemann A. Relevance of claudication pain distance in patients with peripheral arterial occlusive disease VASA. 1999;28:25-29.

16. McGraw KO, Wong SP. Forming inferences about some intraclass correlation coefficients. Psychol Methods. 1996;1:30-46. 
CHAPTER 6

Validation

of the Dutch

version of

the Walking

Impairment

Questionnaire

Marieke Verspaget

Saskia P.A. Nicolaï

Lotte M. Kruidenier

Rob J.Th.J. Welten

Martin H. Prins

Joep A.W. Teijink

Eur J Vasc Endovasc Surg. 2009;37:56-61 


\section{Abstract}

Objectives: The Walking Impairment Questionnaire (WIQ) is a frequently used questionnaire to evaluate patients with intermittent claudication (IC). Aim of this study is to validate the Dutch WIQ for the European situation using the metric system.

Design: Validation study.

Materials: After translation and cultural adaptation of the WIQ, 130 patients with IC completed the Dutch WIQ, the Rand-36, and the EuroQol questionnaire. Walking distances were determined by treadmill testing.

Methods: Correlations between the WIQ, the two quality of life questionnaires, and walking distances were calculated to determine validity. Reliability and internal consistency were determined using the intraclass correlation coefficient (ICC) and Cronbach's alpha, respectively.

Results: Significant correlations were found between the WIQ and the absolute claudication distance (ACD) (0.52), EuroQol (0.33) and 7 domains of the Rand36. Test-retest reliability expressed by the ICC was 0.89 . The internal consistency determined by Cronbach's alpha was 0.92 for the total WIQ score. Furthermore, a lower WIQ score corresponds with a shorter ACD.

Conclusions: This study shows that the Dutch version of the WIQ using the European metric system is a valid, reliable and clinically relevant instrument for assessing walking impairment in patients with intermittent claudication.

\section{Introduction}

Peripheral arterial disease (PAD) is a common manifestation of atherosclerosis affecting the abdominal aorta and peripheral arteries. Classic symptom of symptomatic PAD is intermittent claudication, which describes pain in one or both calves that relieves in rest and starts with walking. Intermittent claudication often results in an impairment of walking ability which can interfere with personal, social or occupational factors in daily life. Usually, treadmill testing is performed to assess the severity of walking impairment.' However, treadmill testing is time consuming, often not available and inconvenient. For this reason, Regensteiner (1990) developed the Walking Impairment Questionnaire (WIQ). ${ }^{2}$ The WIQ is a short, easy to fill out questionnaire and evaluates the degree of walking impairment in patients with intermittent claudication on three domains; walking distance, walking speed and the ability to climb stairs. These three domains represent common daily activities that are frequently impaired in patients with symptomatic PAD and contribute to the degree of walking impairment. The WIQ is validated in the English and Spanish language in United States customary units (distances expressed as feet) ${ }^{2-4}$ Coal of this study is to determine if the culturally adapted WIQ to the European metric situation in the Dutch language is a valid instrument for measuring walking impairment in patients with intermittent claudication.

\section{Methods}

Patients and study design

One hundred and thirty consecutive patients with symptomatic PAD (according to Fontaine stage II) were included from a vascular outpatient clinical setting. Inclusion criteria were patients with a maximal walking distance $<750$ meters assessed by a treadmill test. Exclusion criteria were patients unable to walk on a treadmill, patients with PAD according to Fontaine stage I, III or IV, patients with severe cardiopulmonary co morbidities (NYHA 3 or 4), patients with other co morbidities limiting walking ability or patients with insufficient knowledge of the Dutch language. Informed consent was obtained and the study was approved by the medical ethical committee of the Atrium medical centre Parkstad.

The concurrent validity was determined by comparing the WIQ with the walking distances defined as the absolute claudication distance (ACD) and functional claudication distance

(FCD). The absolute claudication distance represents the maximal possible walking distance. The FCD is defined as the moment the patient prefers to stop and would stop walking in daily life. A treadmill test with a constant speed of $3.2 \mathrm{~km} / \mathrm{h}$, starting at $0 \%$ incline increasing $2 \%$ every two minutes was performed to asses ACD and FCD. ${ }^{5}$ To determine the construct validity patients were asked to fill out the WIQ and two quality of life questionnaires (RAND36, EuroQol). For the test-retest reliability 30 patients filled out a second WIQ two days after filling out the first one.

WIQ

The WIQ is a short, easy to fill out disease specific questionnaire validated in patients with intermittent claudication. ${ }^{2-4}$ It contains three domains measuring three important factors of walking impairment in symptomatic PAD patients; walking distance, walking speed and the ability to walk sWtairs.

The WIQ was translated according to Beaton's guidelines for the process of translation and cross-cultural adaptation of self-report measures. ${ }^{6}$ The WIQ was independently translated to Dutch by two bilingual translators with the Dutch mother tongue and then translated back by two native English-speaking translators. Inconsistencies in translation among translators were resolved by discussion. 
Cultural adaptation was necessary since the American WIQ investigates the walking distance domain with standard sized living blocks as reference for the distance of 300 feet (91.4 meters). In Europe "standard" sized living blocks are not a common phenomenon, which implicates an impaired applicability of the directly translated WIQ regarding the European situation. Furthermore, United States customary units (feet) were translated in meters of the metric system. The authors formed a review committee which examined the translations and agreed on a final version which was cultural applicable and reflected the intent of the instrument. For each domain separately a sub score of the Likert items was calculated with a Likert scale.7 The total WIQ score was the mean of the three subscores.

\section{RAND-36}

The RAND-36 is more commonly known as the Short Form 36 (SF-36), which uses a slightly different scoring algorithm than the SF-36.8 The RAND-36 is a multidimensional generic quality of life questionnaire assessing change in health and eight health domains: physical functioning, social functioning, physical role impairment, emotional role impairment, mental health, vitality, pain and general health experience. The change in health is evaluated in a separate question with standardised response choices. The scores are summed up and transformed to a scale ranging from 0 to 100 for every domain separately. A higher score means a better health condition. In our study a validated Dutch RAND-36 was applied. ${ }^{9-11}$

EuroQol

The EuroQol is a short questionnaire containing five questions with three possible answers. Each question encloses one quality of life dimension: mobility, self care, daily activities (e.g. work, study, domestic and creative activities), pain or other complains and fear or depression. A formula converts the quality of life description in a total quality of life score which is based on judgements of the total general population.'

\section{Measurements methods}

A test is useful when it measures what it is intended to measure (validity) and when the results stay consistent across repeated measurements over time (reliability).

To determine the reliability of the WIQ, internal consistency and test-retest reliability were calculated. The internal consistency measures the degree of homogeneity of a scale based on the correlation between each of the items and the correlation between the items and the total score. The test-retest reliability is estimated by performing the questionnaire twice. The correlation between the two questionnaires is used as a quantitative measure. In this study the patient was asked to fill out a second WIQ two days separated from the first WIQ; a period long enough not to remember the exact answers from the first time and short enough not to expect a therapy effect ${ }^{13}$

Validity was determined by measuring the concurrent and construct validity. Concurrent validity measures the extent to which a new test correlates with a golden standard measuring the same construct, walking impairment. In this study, the correlation between the WIQ and the ACD and FCD assessed by a treadmill test were calculated.

The construct validity is determined if a golden standard is absent and compares the WIQ with other tests measuring the same construct. We compared the WIQ with two quality of life questionnaires, the RAND -36 and EuroQol, measuring the same construct, walking impairment
Statistical analysis

Walking distances and the ankle brachial index (ABI) were presented as median and inter-quartile-range (IQR) since they were not normally distributed. Means are reported to enable the comparison of results of this study with results known from literature. The internal consistency was measured by calculating the Cronbach's alpha. A widely accepted cut- off is set between 0.7 and 0.9 for a set of items to be considered a scale. 14 Test-retest reliability was assessed by the intraclass correlation coefficient (ICC) of absolute agreement based on a two way mixed model with 95\% confidence interval (Cl). 15

Correlations between the WIQ, the two quality of life questionnaires and treadmill walking distances were determined using Spearman correlation coefficients. Statistical analysis was performed with SPSS version 15.0 for windows.

\section{Results}

Study population

One hundred and thirty patients with symptomatic PAD were included in this study with a mean age of 64.5 (SD 10.1). Sixty-three percent of the patients included were male. Forty-four (33.8\%) patients underwent a percutaneous transluminal angioplasty (PTA), 12 (9.2\%) patients received a bypass and $6(4.6 \%)$ patients had a thrombo endarteriectomy (TEA), previously. While several patients underwent more than one vascular intervention, a total of 47 (36.2\%) patients had (multiple) peripheral arteria surgery in their history. The median lowest ABI was 0.70 (IQR 0.57 to 0.83 ) and the median FCD and ACD were 165 (IQR 110 to 230) meters and 240 (IQR 160 to 360) meters, respectively. The mean total score of the WIQ was 0.43 (SD 0.22 ) Other baseline socio-demographic and clinical characteristics of the patients are shown in Table 1. In Figure $1 a$ and $1 b$ the total WIQ scores of the individual patients are plotted against the $A C D$ and the $A B I$, respectively.

Internal consistency and Reliability

At baseline all patients filled out the WIQ with a mean total score of 0.43 (SD 0.22) The internal consistency was 0.91 for the distance domain, 0.81 for the speed domain 0.86 for the stair climbing domain, and 0.92 for the total WIQ score. This implicates a sufficient homogeneity of all the individual domains as well as the total questionnaire. Thirty patients completed the WIQ at baseline and two days later to determine the test-retest reliability expressed by the ICC. For the individual WIQ domains the ICC varies between 0.75 and 0.80 , the ICC of the total WIQ score was highest (0.89) indicating a good test-retest reliability. Table 2 summarizes these results.

\begin{tabular}{|c|c|c|c|c|c|}
\hline WIQ & $\begin{array}{l}\text { Baseline } \\
\text { mean (SD) }\end{array}$ & $\begin{array}{l}\text { Retest } \\
\text { mean (SD) }\end{array}$ & $\begin{array}{l}\text { Change of } \\
\text { scores } \\
\text { mean (SD) }\end{array}$ & $\begin{array}{l}\text { Test-retest } \\
\text { (intraclass correlation } \\
\text { coefficient) }\end{array}$ & $\begin{array}{l}95 \% \\
\text { confidence } \\
\text { interval }\end{array}$ \\
\hline Distance & $0.35(0.26)$ & $0.37(0.25)$ & $0.01(0.18)$ & 0.785 & $0.56-0.90$ \\
\hline Speed & $0.39(0.22)$ & $0.47(0.25)$ & $-0.04(0.15)$ & 0.797 & $0.60-0.91$ \\
\hline Stair climbing & $0.54(0.31)$ & $0.53(0.27)$ & $-0.06(0.19)$ & 0.751 & $0.51-0.88$ \\
\hline Total score & $0.43(0.22)$ & $0.44(0.22)$ & $-0.03(0.11)$ & 0.890 & $0.76-0.95$ \\
\hline
\end{tabular}

WIQ indicates walking impairment questionnaire and SD standard deviation. 


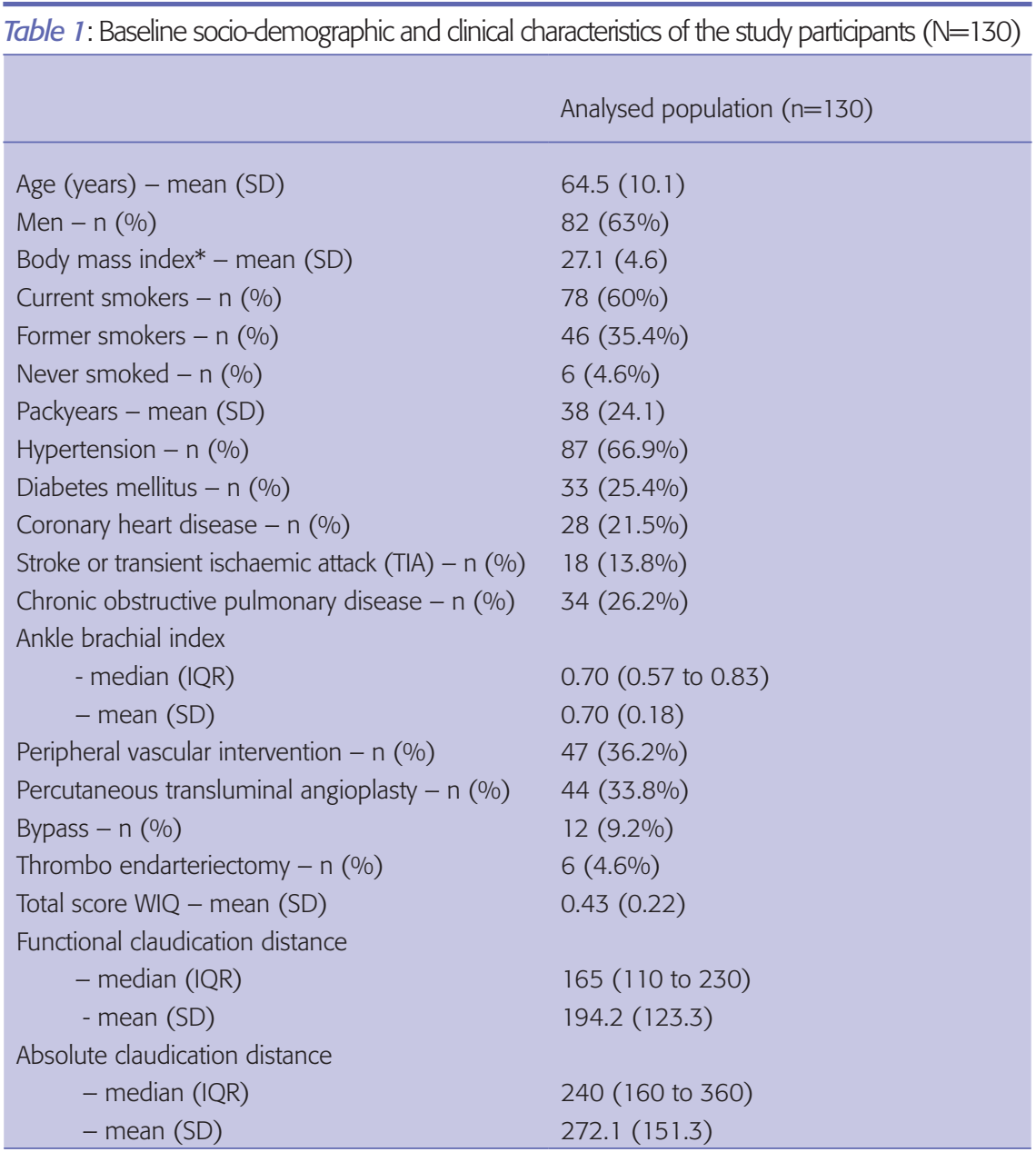

SD indicates standard deviation, IQR interquartile range, and WIQ walking impairment questionnaire. * Body mass index is the weight in kilograms divided by the square of the body height in meters

Figure 1a: The total WIQ score plotted against the absolute claudication distance

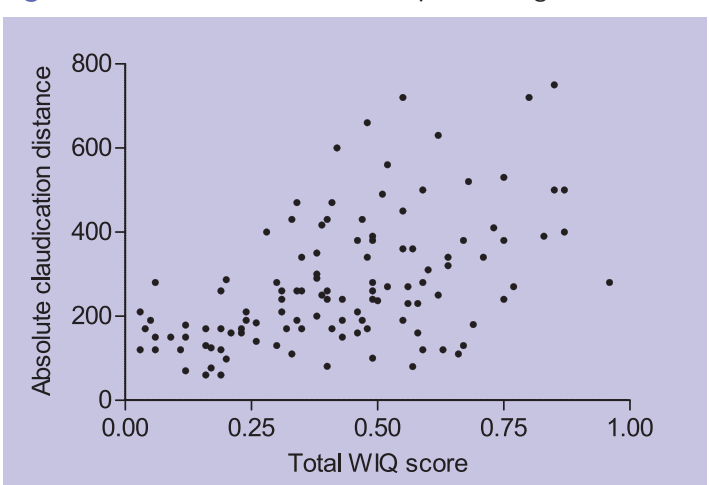

\section{Figure $1 b$ : The total WIQ score plotted against the ankle brachial index}

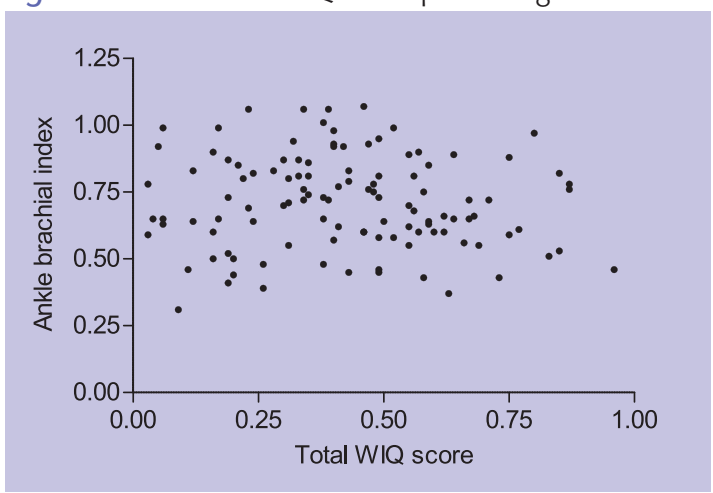

Validity

The concurrent validity as determined by the correlation between the WIQ and the FCD and ACD showed a fairly strong association. The correlation coefficients were 0.52 for the total WIQ score compared with the ACD and 0.48 between the WIQ and the FCD. Correlation coefficients for all WIQ domains are presented in Table 3.

The walking distances in relation to the total WIQ scores divided in tertiles are given in Table 4. Lower scores of the WIQ correspond with shorter walking distances and higher scores with longer walking distances.

The construct validity based on the correlation between the WIQ and other

questionnaires measuring the same construct, showed highest Spearman correlation coefficients between the WIQ and the RAND-36 for the subscales physical functioning (rho 0.70) and pain (rho 0.35). Correlation coefficients between the WIQ and the EuroQol varied between 0.26 and 0.33 . Table 5 presents a detailed overview of the correlations of all WIQ domains, RAND-36 and EuroQol.

Table 3 : Concurrent validity. Spearman correlation coefficients between WIQ, functional claudication distance and absolute claudication distance with $\mathrm{p}$-values $(\mathrm{n}=130)$.

\begin{tabular}{lll} 
WIQ & Functional caludication distance & Absolute claudication distance \\
\hline Distance & $0.43(<.01)$ & $0.45(<.01)$ \\
Speed & $0.45(<.01)$ & $0.43(<.01)$ \\
Stair climbing & $0.32(<.01)$ & $0.37(<.01)$ \\
Total score & $0.48(<.01)$ & $0.52(<.01)$ \\
& &
\end{tabular}

WIQ indicates walking impairment questionnaire 
Table 4: Tertiles of the WIQ compared with the mean functional claudication distance and absolute claudication distance $(n=130)$.

\begin{tabular}{lll} 
WIQ Total score in tertiles & $\begin{array}{l}\text { Functional claudication } \\
\text { distance } \\
\text { (meters) - mean (SD) }\end{array}$ & $\begin{array}{l}\text { Absolute claudication } \\
\text { distance } \\
\text { (meters) - mean (SD) }\end{array}$ \\
\hline $0.00-0.33$ & $128.7(65.3)$ & $178.9(81.9)$ \\
$0.34-0.51$ & $198.1(106.7)$ & $295.0(133.2)$ \\
$0.52-0.96$ & $263.4(161.3)$ & $351.9(181.0)$ \\
\hline
\end{tabular}

WIQ indicates walking impairment questionnaire and SD standard deviation

Table 5: Construct validity. Spearman correlation coefficients between WIQ, EuroQol and RAND with p-values ( $\mathrm{n}=130$ ).

\begin{tabular}{|c|c|c|c|c|c|}
\hline & & WIQ distance & WIQ speed & WIQ stairs & Total \\
\hline \multicolumn{6}{|l|}{ RAND -36 } \\
\hline \multirow[t]{4}{*}{$\begin{array}{l}\text { Functional } \\
\text { status }\end{array}$} & $\begin{array}{l}\text { Physical } \\
\text { functioning }\end{array}$ & $0.513(<.01)$ & $0.557(<.01)$ & $0.615(<.01)$ & $0.697(<.01)$ \\
\hline & Social functioning & $0.091(.32)$ & $0.144(.11)$ & $0.117(.19)$ & $0.108(.25)$ \\
\hline & $\begin{array}{l}\text { Role impairment } \\
\text { (physical) }\end{array}$ & $0.228(.01)$ & $0.281(<.01)$ & $0.244(<.01)$ & $0.273(<.01)$ \\
\hline & $\begin{array}{l}\text { Role impairment } \\
\text { (emotional) }\end{array}$ & $0.140(.13)$ & $0.210(.02)$ & $0.187(.04)$ & $0.197(.03)$ \\
\hline \multirow[t]{3}{*}{ Welbeing } & Mental health & $0.188(.04)$ & $0.191(.04)$ & $0.159(.08)$ & $0.184(.05)$ \\
\hline & Vitality & $0.202(.03)$ & $0.222(.02)$ & $0.241(<.01)$ & $0.251(<.01)$ \\
\hline & Pain & $0.338(<.01)$ & $0.351(<.01)$ & $0.267(<.01)$ & $0.352(<.01)$ \\
\hline $\begin{array}{l}\text { General } \\
\text { evaluation }\end{array}$ & $\begin{array}{l}\text { General health } \\
\text { experience }\end{array}$ & $0.262(<.01)$ & $0.215(.02)$ & $0.169(.06)$ & $0.267(<.01)$ \\
\hline Health & Change in health & $0.165(.07)$ & $0.143(.12)$ & $0.207(.02)$ & $0.181(.05)$ \\
\hline EuroQol & & $0.324(<.01)$ & $0.287(<.01)$ & $0.256(<.01)$ & $0.328(<.01)$ \\
\hline
\end{tabular}

\section{Discussion}

Our results demonstrate that the Dutch version of the WIQ, using the European metric system, is an internally consistent, reliable and valid questionnaire for the evaluation of

walking impairment in patients with intermittent claudication. This implies that the Dutch WIQ can easily (with the forward - backword method according to Beaton) be translated for other European countries without further need for cultural adaptation.

By comparing our results with literature we found that none of the prior studies calculated a total WIQ score, which means there are no directly comparable results available. For the concurrent validity (total WIQ score compared with treadmill testing), we found a correlation coefficient of 0.52 for the ACD. Regensteiner et al. developed and validated the WIQ in the English language in a small population of 26 patients and reported a significant correlation of 0.68 between the distance domain of the WIQ and the ACD. ${ }^{2}$ Quantitative measures (ABI, ACD and self-selected treadmill pace) were compared with qualitative measures (WIQ and SF-36) by Myers et al.16
Correlations of $0.43,0.41$ and 0.39 were reported for the WIQ distance, speed and climbing the stairs domains, respectively in comparison with the ACD. Furthermore, the WIQ is validated in a heterogeneous group of patients with and without PAD and correlations of 0.56 between the WIQ distance score and the 6 minute walk score, and correlations of 0.53 between the WIQ speed score and the 4 minute usual paced score were reported ${ }^{3}$ Possible explanation for the slightly lower correlation coefficients found in our study in comparison with Regensteiner et al. ${ }^{2}$ could be the cultural adaptation in which 'American feet' were translated into meters and where 'standard size living blocks', which are not a common phenomenon in Europe, could not be incorperated in the Dutch questionnaire as a reference for the distance.

Limitation of our study is the fact that the WIQ was compared with walking distance as measured on a treadmill and not with the actual walking distance of a patient in daily life. Although treadmill testing has face validity for walking ability, great variation between the subjective walking distance and the walking distance as measured on a treadmill 17,18 has been demonstrated. Hence, the real golden standard for determining the concurrent validity would be a test with a patient walking on the street. However, the American College of Sports Medicine recommend to evaluate PAD patients with a graded or a gradual ramp protocol on a treadmill.' In our study we partially covered this problem by additionally measuring the construct validity.

The correlation coefficients representing the construct validity between the WIQ and the RAND-36 were 0.70 and 0.35 for the subscales physical functioning and pain, respectively. The correlation between the total WIQ score and the EuroQol was 0.33. We found comparable results for the separate WIQ domains in the literature., ${ }^{416}$ Our study shows that the WIQ can be of use in properly designed clinical trials evaluating therapy effect in PAD patients and in an outpatient clinical setting to evaluate the degree of walking impairment. This is demonstrated by dividing total WIQ scores in tertiles, where lower scores of the WIQ correspond with shorter walking distances and higher scores with longer walking distances. This implies that the WIQ gives an impression of the functional capacity. It would be interesting to know if the Dutch WIQ is able to detect the effect of conservative or invasive therapy.

We conclude that the Dutch version of the WIQ using the European metric system is a valid and reliable instrument for assessing walking impairment in patients with intermittent claudication. 


\section{References}

1. ACSM's guidelines for exercise testing and prescription / American College of Sports Medicine. 7 ed. Baltimore: Lippincott Williams and Wikins; 2006.

2. Regensteiner JG, Steiner JF, Panzer RJ, Hiatt WR. Evaluation of walking impairment by questionnaire in patients with peripheral arterial disease. J Vasc Med Biol. 1990;2142-152.

3. McDermott MM, Liu K, Guralnik JM, Martin GJ, Criqui MH, Greenland P. Measurement of walking endurance and walking velocity with questionnaire: validation of the walking impairment questionnaire in men and women with peripheral arterial disease. J Vasc Surg. 1998;28:1072-1081.

4. Collins TC, Suarez-Almazor M, Petersen N O'Malley KJ. A Spanish translation of the Walking Impairment Questionnaire was validated for patients with peripheral arterial disease. J Clin Epidemiol. Dec 2004;57(12):1305-1315.

5. Gardner AW, Skinner JS, Cantwell BW, Smith LK. Progressive vs single-stage treadmill tests for evaluation of claudication. Med Sci Sports Exerc 1991;23:402-408.

6. Beaton DE, Bombardier C, Guillemin F, Ferraz MB. Guidelines for the process of cross-cultural adaptation of self-report measures. Spine. 2000;25:3186-3191

7. Likert R. Technique for the Measurement of Attitudes. Arch Psychol. 1932;140:1-55.

8. http://unw.sf-36.org/faq/generalinfo.aspx.

9. Ware JE, Jr., Sherbourne CD. The MOS 36-item short-form health survey (SF-36). I. Conceptual framework and item selection. Med Care. 1992;30:473-483.

10. van der Zee KI, Sanderman R. Het meten van de algemene gezondheidstoestand met de RAND-36: een handleiding. Groningen: Noordelijk centrum voor gezondheidsvraagstukken / Rijksuniversiteit Groningen; 1993.
11. Aaronson NK, Muller M, Cohen PD, et al. Translation, validation, and norming of the Dutch language version of the SF-36 Health Survey in community and chronic disease populations. J Clin Epidemiol. 1998;51:1055-1068.

12. EuroQol-a new facility for the measurement of health-related quality of life. The EuroQol Group. Health Policy. 1990;16:199-208.

13. Streiner DL, Norman GR. Health maesurement scales; A practical guide to their development and use. New York: Oxford University Press; 2003.

14. Bland JM, Altman DG. Cronbach's alpha. BMJ. 1997;314:572.

15. McGraw KO, Wong SP. Forming inferences about some intraclass correlation coefficients. Psychological Methods. 1996;1:30-46.

16. Myers SA, Johanning JM, Stergiou N, Lynch TC, Longo $\mathrm{GM}$, Pipinos, II. Claudication distances and the Walking Impairment Questionnaire best describe the ambulatory limitations in patients with symptomatic peripheral arterial disease. J Vasc Surg. 2008;47:550-555.

17. Siggaard-Andersen J, Petersen FB. Intermittent claudication. A comparison between subjective and measured claudication walking distance. Angiology. 1968;19:426-434.

18. Watson CJ, Phillips D, Hands L, Collin J. Claudication distance is poorly estimated and inappropriately measured. Br J Surg. 1997;84:1107-1109.

19. Muller-Buhl U, Kirchberger I, Wiesemann A. Relevance of claudication pain distance in

patients with peripheral arterial occlusive disease. Vasa. 1999:28:25-29

\section{CHAPTER 7}

The Walking

Impairment

Questionnaire:

an effective

tool to assess

the effect of

treatment

in patients

with intermittent

claudication

\author{
Saskia P.A. Nicolaï \\ Lotte M. Kruidenier \\ Ellen V. Rouwet \\ Kisten Graffius \\ Martin H. Prins \\ Joep A.W. Teijink
}

J Vasc Surg. 2009;50:89-94 


\section{Abstract}

Objective: Assessment of walking distance by treadmill testing is the most commonly used method to evaluate the effect of treatment in patients with peripheral arterial disease. However, treadmill testing is time consuming, relatively expensive and does not adequately reflect real life functional ability. We hypothesized that the Walking Impairment Questionnaire (WIQ) could be an alternative tool to assess objective improvement in functional walking ability of patients with intermittent claudication.

Methods: This was a validation study. It was conducted through the outpatient clinic for vascular surgery. Patients with intermittent claudication were referred for supervised exercise therapy. Treadmill testing (absolute claudication distance $[A C D]$ ), WIQ, and quality of life questionnaires (RAND-36 and EuroQol) were administered at study onset and after 3 months of supervised exercise therapy. Responsiveness was determined by mean changes in and correlation coefficients of WIQ, ACD, and quality of life questionnaires. Patients were categorized into quartiles based on the increase in $A C D$, which were subsequently related to change in WIQ and quality of life.

Results: The mean pre- and post-treatment total WIQ scores of 91 patients were $0.45(0.22)$ and $0.58(0.22)$, respectively. The correlation coefficient between the change in total WIQ score and ACD was $0.331(P=.004)$. A 0.1 change in total WIQ score corresponded to a change of 345 meters in ACD. Analysis of the four quartiles compared to an increase in ACD showed that a greater increase in ACD corresponded with a greater increase in WIQ score, from 0.06 to $0.25(P=.011)$.

Conclusion: These data indicate that the WIQ is a valid tool to detect improvement or deterioration in the daily walking ability of patients with intermittent claudication. Hence, the WIQ can be used as an alternative to treadmill testing for objective assessment of functional walking ability, both in daily practice and in clinical trials.

\section{Introduction}

Intermittent claudication decreases functional capacity, walking ability' and quality of life.2 It is increasingly recognized that assessment of functional capacity and walking ability are important in determining disease severity and evaluating treatment in patients with intermittent claudication. Treadmill testing is currently the most commonly used measure to assess walking ability as expressed in meters. However, a large discrepancy often exist among subjectively experienced daily walking ability ${ }^{3,4}$, objective severity of the disease ${ }^{5}$ and walking ability as measured on a treadmill in a vascular laboratory. Furthermore, treadmill testing requires adequate equipment and personnel, is time consuming, and is relatively expensive. A qualitative approach to document daily walking ability is the Walking Impairment Questionnaire $(\mathrm{WIQ})^{7}$, a validated questionnaire which is short, easy to complete, and inexpensive. The questionnaire evaluates walking ability with a focus on walking distance, walking speed and the ability to climb stairs., 9 The WIQ has been used in studies to evaluate the effect of treatment in patients with peripheral arterial disease. ${ }^{10-12}$ Furthermore, one study showed that the WIO is also capable of gauging cardiovascular risk status in patients with peripheral arterial disease. ${ }^{13} \mathrm{~A}$ recent study suggests that the WIQ is the most specific questionnaire to evaluate walking ability in patients with peripheral arterial disease, and that the WIQ correlates best with the ambulatory limitations as measured on a treadmill. ${ }^{14}$ However, more important is the association between a change in WIQ score and a clinical improvement or deterioration in walking ability in assessing the effect of treatment. In this study we hypothesized that the WIQ, which can be administered within 5 minutes and inexpensively, can be used as an alternative tool to detect objective improvement in functional ability in response to supervised exercise therapy in patients with intermittent claudication.

\section{Methods}

Patients

Consecutive patients presenting at the outpatient clinic for vascular surgery with intermittent claudication (Rutherford stage 1-3 or Fontaine stage II) were included in the study. Diagnosis of intermittent claudication was confirmed with an $A B I$ measurement $<0.9$ at rest or decreasing $>0.15$ after exercise. ${ }^{15}$ Inclusion criteria were the ability to walk on a treadmill (3.2 $\mathrm{km} / \mathrm{h}$ ) before treatment was initiated as well as a maximal walking distance of 750 meters as assessed on a treadmill. Exclusion criteria was cessation of the treadmill test due to factors other than intermittent claudication, e.g. a cardiopulmonary or orthopaedic disorder that limited the walking distance. Informed consent was obtained from all patients, and the study was approved by the medical ethics committee of the Atrium Medical Centre Parkstad.

\section{Supervised exercise therapy}

For symptomatic relief patients were treated with supenised exercise therapy. ${ }^{16,17}$ Exercise therapy is recommended as the primary mode of treatment for patients with intermittent claudication ${ }^{15}$, and supervised exercise therapy has been shown to have an additional benefit over non-supenvised exercise therapy in improving walking distance..$^{18} \mathrm{All}$ patients were referred to a physiotherapist participating in the Network for Exercise Therapy Parkstad (NETP). ${ }^{16}$ The NETP provides community-based supervised exercise therapy in private physiotherapy practices equally distributed over the region, instead of a department of physiotherapy and rehabilitation in a hospital or rehabilitation clinic. This approach has been demonstrated to be as equally effective in providing symptomatic improvement as a clinic-based approach ${ }^{17}, 19$, with the additional advantage of close proximity to the patient's home address. Furthermore, all patients received a platelet inhibitor, cholesterol lowering medication, and smoking cessation and lifestyle advice by a trained nurse practitioner as part of the standard treatment protocol. 
The WIQ, treadmill testing and administration of quality of life questionnaires were administered at the start of the study and after 3 months of supervised exercise therapy. The WIQ is a short, easy to fill out, disease-specific questionnaire validated in patients with intermittent claudication..$^{-9}$ We used the revised version of the WIQ ${ }^{9}$, that has recently been adapted and validated for the European metric system and specifically the Dutch language. ${ }^{20}$ The questionnaire was self-administered by the patients, as validated by Coyne ${ }^{9}$, and contains three domains measuring three important factors of walking impairment in patients with intermittent claudication: walking distance, walking speed and the ability to climb stairs. For each separate domain a subscore of the Likert items was calculated with a Likert scale. The total WIQ score was defined as the mean of the three subscores, and was recently validated. ${ }^{20}$

Treadmill testing

To assess walking distance, all patients performed a standardized graded treadmill test with a constant speed of $3.2 \mathrm{~km} / \mathrm{h}$, starting at $0 \%$ incline and increasing $2 \%$ every two minutes. ${ }^{21}$ For practical reasons, during the follow-up measurements the inclination and duration of the test were maximized to 10\% and 30 minutes (1600 meters), respectively. Test variables during treadmill testing were the absolute claudication distance (ACD) defined as the distance that severe claudication pain forces cessation of exercise, and the functional claudication distance (FCD), which is the moment the patient prefers to stop due to claudication pain.

Quality of life questionnaires

Quality of life was assessed with the RAND-36 and the European Quality of life (EuroQol) instruments. The RAND-36 is a generic quality of life instrument that provides an 8-scale profile of functional health and well-being. It is an exact replica of the Short Form 36 (SF-36), except for use of a different scoring algorithm.22 The 8 domains of assessing quality of life are physical functioning, social functioning, physical role impairment, emotional role impairment, mental health, vitality, pain and general health experience. ${ }^{23}$ Scores range between 0 and 100, with 0 expressing the worst health status and 100 the best. We used the validated Dutch RAND-36 for evaluation in this study. ${ }^{24}$ The EuroQol, a two-part questionnaire, is designed to measure general health ${ }^{25}$ and consists of an overall health classification index containing five questions to evaluate physical functioning (mobility, self-care, usual activities, pain/discomfort, and anxiety/depression). The EuroQol classifies patients into a health state which is calculated with an algorithm ranging from 0 to $1{ }^{26}$ Furthermore, health status was assessed by a visual analogue scale (VAS), with 0 expressing the worst health state and 100 the best.

Statistical analysis

Walking distances are presented as median and interquartile-range (IQR) since they were not normally distributed. The statistical significance of the WIQ was analyzed with a paired student's t-test, and walking distances were analyzed with a Wilcoxon Signed Ranks Test. To evaluate the ability of the WIQ to detect objective improvement, we calculated mean changes between baseline and 3 months of supervised exercise therapy with respect to the WIQ, walking distances (FCD and ACD), and generic quality of life questionnaires. Correlations were calculated with the Spearman correlation coefficients. Univariate linear regression analysis was used to demonstrate the dependency of the change in total WIQ score on the change in ACD.
We next determined whether the change in WIQ at 3 months follow-up was related to the change in walking distance (ACD). Since it is inappropriate to use summary statistics on percentage changes due to improper cancellation of positive and negative changes ${ }^{27}$ we used a linear regression model to categorize the response to treatment. To this end, the expected ACD at 3 months for each patient was calculated with univariate linear regression analysis, with baseline ACD as the independent variable. For each individual, the difference between expected and actually measured ACD at 3 months was calculated. These calculated differences for the entire study population were divided into quartiles, and patients were correspondingly categorized into 4 responder groups. Changes in WIQ, RAND-36 and EuroQol were related to the responder groups. One-way ANOVA testing was performed to analyze the changes in WIQ, RAND-36 and EuroQol for the 4 responder groups, i.e. to determine whether improvement in these scores correlated with improvement in walking distance. Analyses were performed using SPSS 15.0 for Windows.

\section{Results}

This study included 91 consecutive patients with a mean age of 66.2 years. All patients were asked to fill out a self-administered WIQ; the mean ABI was 0.72 at rest. Baseline characteristics of the study population are presented in Table 1 .

\begin{tabular}{ll}
\hline \multicolumn{2}{l}{ Table 1: Baseline characteristics of the study population } \\
\hline & Study population $\mathrm{n}=91$ \\
\hline & $66.2(9.6)$ \\
Age - mean (SD) & 61.5 \\
Men - \% & $27.6(4.7)$ \\
BMI- mean (SD) & $0.72(0.17)$ \\
Resting ABI - mean (SD) & \\
Smoking & 6.6 \\
Never - \% & 39.6 \\
Stopped smoking - \% & 53.8 \\
Current smoker - \% & 64.8 \\
Hypertension - \% & 66.7 \\
Hypercholesterolaemia - \% & 26.4 \\
Diabetes mellitus - \% & 19.8 \\
Coronary heart disease - \% & 16.5 \\
Stroke or TIA - \% & 31.9 \\
COPD - \% &
\end{tabular}

$B M I$ indicates body mass index, ABI ankle brachial index, TIA transient ischaemic attack, and COPD chronic obstructive pulmonary disease

WIQ and walking distances

The total WIQ score as well as the score for each of the three WIQ subdomains were significantly higher at three months after initiation of supervised exercise therapy as compared with baseline (Table 2), indicating an improvement in functional walking ability as assessed by the questionnaire. The mean change in total WIQ score was 0.14 (SD 0.19). Walking distances as assessed by treadmill testing in the vascular laboratory also significantly improved. After 3 months of supervised exercise therapy, walking distance of 11 out of 91 patients was truncated by the maximal duration of 30 minutes of the treadmill test (1600 meters). 
The FCD improved from 179 to 330 meters (median), while the ACD improved from 245 to 460 meters (median) $(\mathrm{P}<.001)$. The correlation between the increase in WIQ and increase in ACD is presented in Figure 1. The Spearman correlation coefficient between the change in total WIQ score and the change in ACD was $0.331(\mathrm{P}=.004)$. Furthermore, univariate linear regression analysis showed a dependency of the change in total WIQ score on the change in $\mathrm{ACD}(\mathrm{P}<.001)$. A change of 0.1 in total WIQ score corresponded to a change in ACD of 345 meters.

\begin{tabular}{|c|c|c|c|c|c|}
\hline & & Pre-treatment & Post-treatment & P-value & Change \\
\hline \multirow[t]{5}{*}{ WIQ } & & Mean (SD) & Mean (SD) & & Mean (SD) \\
\hline & Distance & $0.38(0.26)$ & $0.55(0.29)$ & $<.001 *$ & $0.18(0.28)$ \\
\hline & Speed & $0.41(0.22)$ & $0.52(0.22)$ & $<.001^{*}$ & $0.11(0.20)$ \\
\hline & Stair climbing & $0.55(0.32)$ & $0.68(0.29)$ & $<.001^{*}$ & $0.14(0.29)$ \\
\hline & Total score & $0.45(0.22)$ & $0.58(0.22)$ & $<.001^{*}$ & $0.14(0.19)$ \\
\hline \multirow[t]{3}{*}{$\begin{array}{l}\text { Walking } \\
\text { distances }\end{array}$} & & Median (IQR) & Median (IQR) & & Median (IQR) \\
\hline & FCD & $\begin{array}{l}179 \\
\text { (120 to } 260 \text { ) }\end{array}$ & $\begin{array}{l}330 \\
\text { (223 to } 480 \text { ) }\end{array}$ & $<.001^{* *}$ & $\begin{array}{l}135 \\
\text { (40 to } 290 \text { ) }\end{array}$ \\
\hline & $\mathrm{ACD}$ & $\begin{array}{l}245 \\
\text { (168 to } 380)\end{array}$ & $\begin{array}{l}460 \\
\text { (308 to 593) }\end{array}$ & $<.001^{* *}$ & $\begin{array}{l}200 \\
(60 \text { to } 340 \text { ) }\end{array}$ \\
\hline
\end{tabular}

FCD indicates functional claudication distance andACD absolute claudication distance

* Paired student's t-test ** Wilcoxon Signed Ranks Test

Figure 1: Change in WIQ compared to the change in absolute claudication distance 3 months after treatment of intermittent claudication.

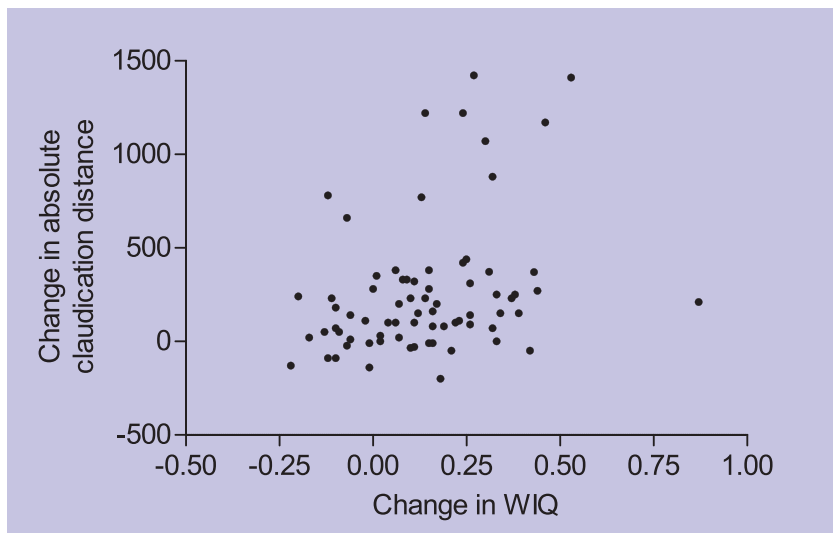

The Walking Impairment Questionnaire: an effective tool to assess the effect of treatment in patients wit intermittent claudiation
WIQ and quality of life

Results for the RAND-36 and EuroQol pre- and post-treatment are shown in Table 3. For each of the subdomains of the RAND-36 and the EuroQol, a higher score indicates an improvement. The largest effects of treatment were found for the subdomains physical functioning and pain of the RAND-36, with mean changes of 7.9 and 7.7, respectively, from pre-treatment to post-treatment (Table 3). The index score of the EuroQol increased from 0.64 to 0.70 . No significant correlation between the change in subdomains of the RAND36 or EuroQol scores and the change in total WIQ score was found.

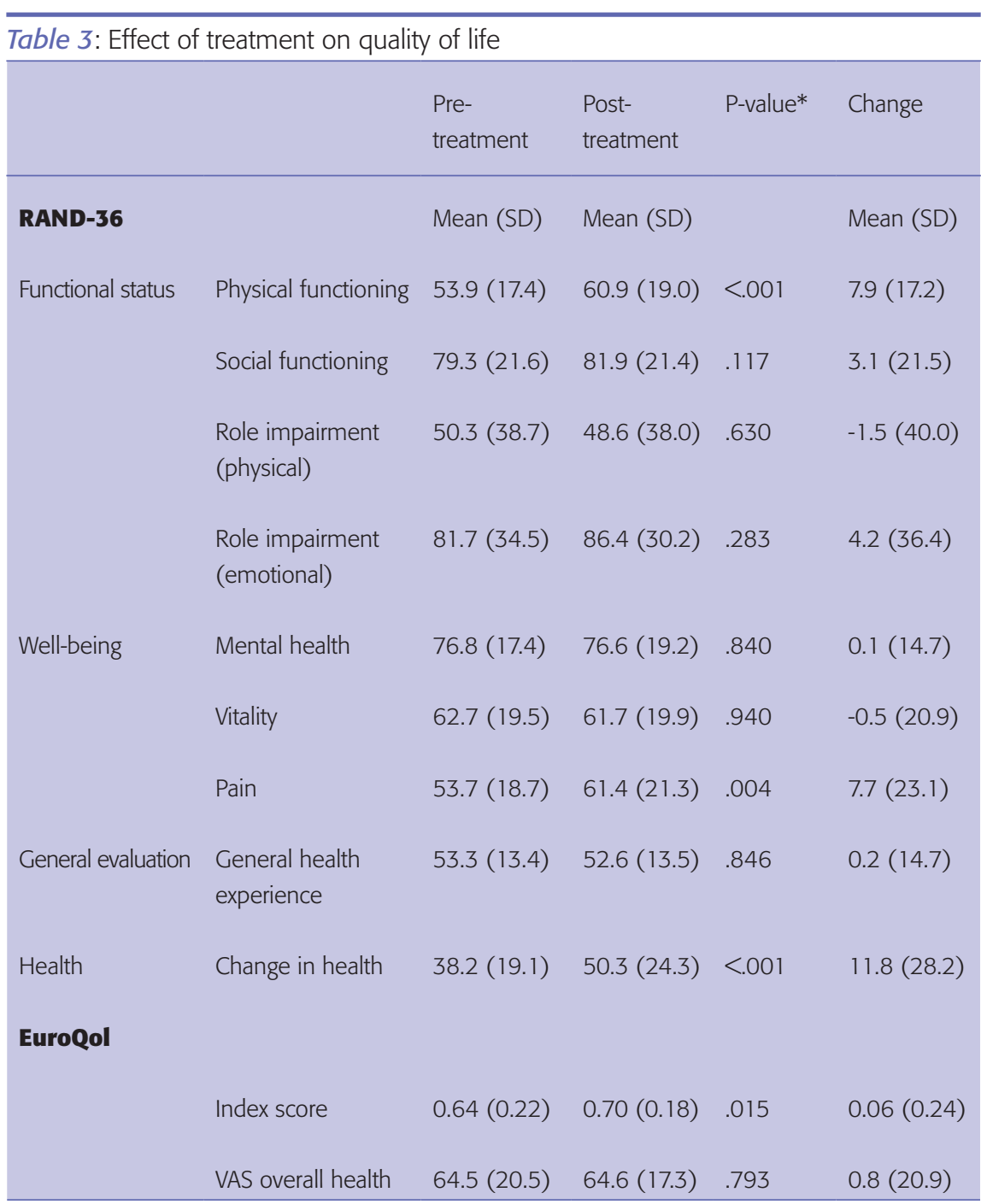

Practical implications

Patients were divided into responder groups based on the difference between expected and measured ACD post-treatment. In Table 4, quartiles based on this difference were related to the change in WIQ. In the first quartile the response was 248 meters or less than expected in the second quartile the response ranged between 248 and 108.5 meters less than expected, in the third quartile the response was between 108.5 meters less and 62 meters 
more than expected, and in the fourth quartile the response was more than 62 meters than expected. Over these four quartiles, the total WIQ scores significantly improved from 0.06 to $0.25(\mathrm{P}=.011)$. This indicates that a qualitative improvement in walking ability as assessed by the questionnaire shows good correspondence with a quantitative improvement in walking distance as assessed by treadmill testing. In line with this, the subdomain physical functioning of the RAND-36 showed a (non-significant) increase from 2.8 to 13.0 ( $P=.118)$.

\section{Table 4: Relation between qualitative improvement in walking ability as assessed} by questionnaires and quantitative improvement in walking distance as assessed by treadmill testing

\begin{tabular}{|c|c|c|c|c|c|c|}
\hline & & $\begin{array}{l}\text { Quartile } 1 \\
\text { Response } \\
248 \text { meters } \\
\text { or less than } \\
\text { expected }\end{array}$ & $\begin{array}{l}\text { Quartile } 2 \\
\text { Response } \\
\text { between } \\
248 \text { to } \\
108.5 \\
\text { meters } \\
\text { less than } \\
\text { expected }\end{array}$ & $\begin{array}{l}\text { Quartile } 3 \\
\text { Response } \\
\text { between } \\
108.5 \text { less } \\
\text { and } 62 \\
\text { meters } \\
\text { more than } \\
\text { expected }\end{array}$ & $\begin{array}{l}\text { Quartile } 4 \\
\text { response } \\
62 \text { meters } \\
\text { or more } \\
\text { than } \\
\text { expected }\end{array}$ & $\begin{array}{l}\text { P- } \\
\text { value* }\end{array}$ \\
\hline \multicolumn{7}{|l|}{ WIQ } \\
\hline & Distance & $0.12(0.27)$ & $0.11(0.25)$ & $0.18(0.33)$ & $0.30(0.24)$ & .113 \\
\hline & Speed & $0.04(0.20)$ & $0.08(0.19)$ & $0.13(0.22)$ & $0.23(0.17)$ & .016 \\
\hline & Stair climbing & $0.04(0.27)$ & $0.10(0.20)$ & $0.20(0.38)$ & $0.22(0.27)$ & .152 \\
\hline & Total score & $0.06(0.17)$ & $0.10(0.14)$ & $0.18(0.25)$ & $0.25(0.18)$ & .011 \\
\hline \multicolumn{7}{|l|}{ RAND-36 } \\
\hline \multirow[t]{4}{*}{$\begin{array}{l}\text { Functional } \\
\text { status }\end{array}$} & $\begin{array}{l}\text { Physical } \\
\text { functioning }\end{array}$ & $2.8(17.1)$ & $7.4(16.1)$ & $13.5(15.5)$ & $13.0(16.3)$ & .118 \\
\hline & $\begin{array}{l}\text { Social } \\
\text { functioning }\end{array}$ & $1.1(24.4)$ & $3.1(20.2)$ & $4.2(22.5)$ & $3.6(19.4)$ & .971 \\
\hline & $\begin{array}{l}\text { Role impairment } \\
\text { (physical) }\end{array}$ & $-18.2(45.1)$ & $-1.3(34.8)$ & $13.2(38.5)$ & $3.6(34.7)$ & .075 \\
\hline & $\begin{array}{l}\text { Role impairment } \\
\text { (emotional) }\end{array}$ & $9.1(40.1)$ & $3.3(28.4)$ & $11.7(37.9)$ & $-6.3(30.9)$ & .354 \\
\hline \multirow[t]{3}{*}{ Well-being } & Mental health & $-3.2(19.6)$ & $0.8(13.7)$ & $5.2(13.2)$ & $-0.8(11.1)$ & .324 \\
\hline & Vitality & $-11.1(19.9)$ & $6.3(21.3)$ & $4.0(23.9)$ & $2.4(15.6)$ & .028 \\
\hline & Pain & $-0.4(22.0)$ & $4.6(20.2)$ & $13.9(21.0)$ & $6.8(23.3)$ & .197 \\
\hline $\begin{array}{l}\text { General } \\
\text { evaluation }\end{array}$ & $\begin{array}{l}\text { General health } \\
\text { experience }\end{array}$ & $-5.7(16.1)$ & $-2.4(9.8)$ & $7.4(12.9)$ & $2.5(14.1)$ & .014 \\
\hline \multirow[t]{3}{*}{$\begin{array}{l}\text { Health } \\
\text { EuroQol }\end{array}$} & Change in health & $-3.4(25.9)$ & $26.3(26.3)$ & $21.3(26.0)$ & $13.6(21.4)$ & .001 \\
\hline & Index score & $-0.06(0.18)$ & $0.14(0.22)$ & $0.10(0.21)$ & $\begin{array}{l}0.00 \\
(0.25)\end{array}$ & .022 \\
\hline & $\begin{array}{l}\text { VAS overall } \\
\text { health }\end{array}$ & $-3.2(23.9)$ & $9.3(16.6)$ & $-1.4(27.5)$ & $-1.0(14.5)$ & .326 \\
\hline
\end{tabular}

Values are presented as mean change with standard deviations

*One way ANOVA analysis

\section{Discussion}

The aim of the study was to determine whether the Walking Impairment Questionnaire can be used as an alternative tool to treadmill testing in assessing improvement in functional ability in patients with intermittent claudication treated with supervised exercise therapy. The findings in the present study show that the WIQ is a reliable instrument and alternative to treadmill testing in determining the effect of treatment in patients with intermittent claudication.

Our data demonstrate that patients with intermittent claudication treated with supervised exercise therapy for three months show a gradual increase in their WIQ score; furthermore, the higher the WIQ score, the better patient's functional walking ability. We correlated the WIQ score with the absolute claudication distance to demonstrate that an increase in WIQ corresponds to an increase in walking distance in meters as assessed by the gold standard treadmill test. The correlation coefficient of 0.331 may appear to be modest; however, similar studies comparing objective and subjective instruments to assess the effect of an intervention do not closely correlate. ${ }^{11,28}$ The three statistical methods, including Spearman correlation, linear regression analysis, and responder group analysis, support a similar conclusion, showing that a qualitative improvement in walking ability as assessed by the questionnaire corresponds with a quantitative improvement in walking distance as assessed by treadmill testing. Even more important than the correlation coefficient, responder group analysis showed the ability of the WIQ to detect an effect of treatment. The findings in the present study, therefore, justify the use of the total WIQ score as an alternative to treadmill testing as a primary outcome measurement to assess the efficacy of supervised exercise therapy in patients with intermittent claudication. In line with this, the WIQ is also expected to be a useful tool for assessment of the efficacy of interventions that deliver more direct benefits (e.g. percutaneous vascular interventions or vascular surgery).

In trials, the success of therapies and comparison among treatments has traditionally been determined by treadmill testing or measurement of ankle brachial indices

$(\mathrm{ABI})$. Do we, then, need an alternative instrument to assess efficacy of treatment? Although treadmill testing has been the gold standard for measuring walking distance in meters ${ }^{29}$, this method has several important limitations. Treadmill testing has face validity for functional walking distance; however, a large variation has been reported regarding daily walking ability and claudication walking distances as measured on a treadmill, 3,4 resulting in an unrealistic representation of daily life walking ability. Furthermore, treadmill testing is time consuming, relatively expensive, requires adequate equipment and personnel, and is not directly available for all physicians. Another disadvantage of treadmill testing is that the measured walking distance is dependent on applied treadmill protocols, which are not uniform and therefore hamper its use in the comparison of the effects of treatments, e.g. in clinical trials. Other methods to evaluate walking distances without the use of a treadmill include the 6-minute walking test $^{30-32}$, a shuttle walking test ${ }^{31,33}$, a PADHOC device ${ }^{34}$, or the use of a GPS device. ${ }^{12} \mathrm{~A}$ recent study indicated that performance on corridor based functional measures (e.g. a 6-minute walking test) is more closely linked to physical activity during daily life than treadmill walking performance. ${ }^{32}$ Second, with regard to the effectiveness of the $A B I$ to determine the effect of treatment in patients with intermittent claudication, studies have shown that a significant improvement in walking ability is not necessarily associated with an increase in $\mathrm{ABI}$ after supervised exercise therapy.5 
To overcome the aforementioned limitations, the WIQ was developed. The WIQ is designed as a disease-specific questionnaire that assesses walking ability in patients with peripheral arterial disease. Use of the WIQ in evaluation and assessment has several benefits. First, the WIQ is short, easy to fill out, and inexpensive. The original version of the WIQ was an interviewer-administered questionnaire ${ }^{8}$, and for practical reasons, it has been modified to a validated self-administered or telephoneadministered questionnaire. ${ }^{9}$ Second, the WIQ assesses functional walking ability, including walking distance, walking speed and the ability to climb stairs. These items more realistically reflect a patient's daily life walking capacity than treadmill testing.

The improvement in functional walking ability after three months of supervised exercise therapy was not reflected by an improvement in quality of life, as assessed by two generic quality of life questionnaires. Although the RAND-36 and EuroQol are the most widely used, generic quality of life questionnaires, disease-specific questionnaires, such as the CLAUS and VascuQol, may be more appropriate to determine the

responsiveness to treatment in patients with peripheral arterial disease. ${ }^{36,37}$ Therefore, the use of the WIQ for quality of life assessment will be subject of future studies.

In conclusion, our findings demonstrate that the total WIQ score can be used as an alternative to treadmill testing for objective assessment of functional walking ability in patients with intermittent claudication, both in daily practice and in clinical trials.

\section{References}

1. McDermott MM, Greenland P, Liu K, Guralnik JM, Criqui MH, Dolan NC, et al. Leg symptoms in peripheral arterial disease: associated clinica characteristics and functional impairment. JAMA 2001;286:1599-1606.

2. Dumville JC, Lee AJ, Smith FB, Fowkes FC The health-related quality of life of people with peripheral arterial disease in the community:

the Edinburgh Artery Study. Br J Gen Pract 2004;54:826-831.

3. Siggaard-Andersen J, Petersen FB. Intermittent claudication A comparison between subjective and measured claudication walking distance. Angiology. 1968;19:426-434.

4. Watson CJ, Phillips D, Hands L, Collin J. Claudication distance is poorly estimated and inappropriately measured. Br J Surg. 1997;84:1 107-1 109.

5. Muller-Buhl U, Kirchberger I, Wiesemann A. Relevance of claudication pain distance in patients with peripheral arterial occlusive disease. VASA. 1999;28:25-29.

6. Gardner AW, Montgomery PS, Afaq A. Exercise performance in patients with peripheral arterial disease who have different types of exertional leg pain. J Vasc Surg. 2007:46:79-86

7. McDermott MM, Liu K, Guralnik JM, Martin GJ, Criqui MH, Greenland P. Measurement of walking endurance and walking velocity with questionnaire: validation of the walking impairment questionnaire in men and women with peripheral arterial disease. J Vasc Surg. 1998;28:1072-1081.

8. Regensteiner JG, Steiner JF, Panzer RJ, Hiatt WR. Evaluation of walking impairment by questionnaire in patients with peripheral arterial disease. J Vasc Med Biol. 1990:2:142-152.

9. Coyne KS, Margolis MK, Gilchrist KA, Grandy SP, Hiatt WR, Ratchford A, et al. Evaluating effects of method of administration on Walking Impairment Questionnaire. J Vasc Surg. 2003;38:296-304
10. Hiatt WR, Regensteiner JG, Hargarten ME, Wolfel EE, Brass EP. Benefit of exercise conditioning for patients with peripheral arterial disease

Circulation. 1990;81:602-609.

11. Regensteiner JG, Steiner JF, Hiatt WR. Exercise training improves functional status in patients with peripheral arterial disease. J Vasc Surg. 1996;23:104-115.

12. Le Faucheur A, Abraham P, Jaquinandi V, Bouye P, Saumet JL, Noury-Desvaux B. Measurement of walking distance and speed in patients with periphera arterial disease: a novel method using a global positioning system. Circulation. 2008; 17:897-904.

13. Schiano V, Brevetti G, Sirico G, Silvestro A, Giugliano G, Chiariello M. Functional status measured by walking impairment questionnaire and cardiovascular risk prediction in periphera arterial disease: results of the Peripheral

Arteriopathy and Cardiovascular Events (PACE) study. Vasc Med. 2006; 11:147-154.

14. Myers SA, Johanning JM, Stergiou N, Lynch TC Longo GM, Pipinos II. Claudication distances and the Walking Impairment Questionnaire best describe the ambulatory limitations in patients with symptomatic peripheral arterial disease. IVasc Surg. 2008:47:550-555.

15. Norgren L, Hiatt WR, Dormandy JA, Nehler MR, Harris KA, Fowkes FG. Inter-Society Consensus for the Management of Peripheral Arterial Disease (TASC II). J Vasc Surg. 2007;45 Suppl 5:S5-67.

16. Willigendael $E M$, Bendermacher $B L$ van der Berg C, Welten RJ, Prins MH, de Bie RA, et al. The development and implementation of a regional network of physiotherapists for exercise therapy in patients with peripheral arterial disease, a preliminan report. BMC Health Serv Res. 2005:5:49.

17. Kruidenier LM, Nicolaï SP, Hendriks EJ, Bollen EC, Prins MH, Teijink JA. Supervised exercise therapy for intermittent claudication in daily practice; One year results of a community-based approach. In press J Vasc Surg. 2009. 

non-supervised exercise therapy for intermittent claudication. Cochrane Database Syst Rev. 2006(2):CD005263.

19. Bendermacher BL, Willigendael EM, Nicolai SP, Kruidenier LM, Welten RJ, Hendriks E, et al. Supervised exercise therapy for intermittent claudication in community-based setting is as effective as clinicbased. J Vasc Surg. 2007;45:1 192-1196.

20. Verspaget M, Nicolai SP, Kruidenier LM, Welten RJ, Prins MH, Teijink JA. Validation of the Dutch version of the Walking Impairment Questionnaire. Eur J Vasc Endovasc Surg. 2009;37:56-61.

21. Gardner AW, Skinner JS, Cantwell BW, Smith LK. Progressive vs single-stage treadmill tests for evaluation of claudication. Med Sci Sports Exerc. 1991;23:402-408.

22. http://www.sf-36.org/faq/generalinfo.aspx.

23. Ware JE, Jr, Sherbourne CD. The MOS 36-item shortform health survey (SF-36). I. Conceptual framework and item selection. Med Care 1992:30:473-483.

24. Aaronson NK, Muller M, Cohen PD, Essink-Bot ML, Fekkes M, Sanderman R, et al. Translation, validation, and norming of the Dutch language version of the SF-36 Health Survey in community and chronic disease populations. J Clin Epidemiol. 1998;51:1055-1068

25. EuroQol-a new facility for the measurement of health-related quality of life. The EuroQol Group. Health Policy. 1990;16:199-208.

26. Dolan P. Modeling valuations for EuroQol health states. Med Care. 1997;35:1095-1 108

27. http://biostatmc.vanderbiltedu/twik/bin/view/ Main/ManuscriptChecklist

28. Regensteiner JG, Ware JE, Jr, McCarthy WJ, Zhang P, Forbes WP, Heckman $\downarrow$, et al Effect of cilostazol on treadmill walking, community-based walking ability, and health-related quality of life in patients with intermittent claudication due to peripheral arteria disease meta-analysis of six randomized controlled trials. J Am Geriatr Soc. 2002;50:1939-1946.

29. ACSM's guidelines for exercise testing and prescription / American College of Sports Medicine Baltimore: Lippincott Williams and Wilkins 2006

30. Montgomery PS, Gardner AW. The clinical utility of a six-minute walk test in peripheral arterial occlusive disease patients. J Am Geriatr Soc. 1998;46:706-711.

31. da Cunha-Filho IT, Pereira DA, de Carvalho AM, Campedeli L, Soares M, de Sousa Freitas J. The reliability of walking tests in people with claudication. Am J Phys Med Rehabi 2007;86:574-582

32. McDermott MM, Ades PA, Dyer A, Guralnik JM Kibbe M, Criqui MH. Corridor-based functiona performance measures correlate better with physical activity during daily life than treadmill measures in persons with peripheral arterial disease. J Vasc Surg. 2008;48:1231-7

33. Zwierska I, Nawaz S, Walker RD, Wood RF, Pockley AG, Saxton JM. Treadmill versus shuttle walk tests of walking ability in intermittent claudication. Med Sci Sports Exerc. 2004:36:1835-1840.

34. Coughlin PA, Kent PJ, Turton EP, Byrne P, Berridge BC, Scott DJ, et al. A new device for the measurement of disease severity in patients with intermittent claudication. Eur J Vasc Endovasc Surg. 2001;22:516-522.

35. Watson L, Ellis B, Leng GC. Exercise for intermittent claudication. Cochrane Database Syst Rev. 2008(4):CD000990.

36. Mehta T, Venkata Subramaniam A, Chetter I, McCollum P. Assessing the validity and responsiveness of disease-specific quality of life instruments in intermittent claudication. Eur J Vasc Endovasc Surg. 2006:31:46-52.

37. de Vries M, Ouwendijk R, Kessels AG, de Haan MW, Flobbe K, Hunink MG, et al. Comparison of generic and disease-specific questionnaires for the assessment of quality of life in patients with peripheral arterial disease. J Vasc Surg. 2005;41:261-268.

\section{CHAPTER 8}

Supervised

exercise therapy for intermittent claudication in daily practice:

\section{one-year results of a} communitybased approach
Lotte M. Kruidenier Saskia P.A. Nicolai

Erik J.M. Hendriks Ewald C.M. Bollen Martin H. Prins

Joep A.W. Teijink

Martin H. Prins

J Vasc Surg. 2009;49:263-70 


\section{Abstract}

Objective: This study describes the results and functioning of community-based supervised exercise therapy (SET) at one year of follow-up.

Methods: We conducted a prospective cohort study of community-based SET in regional physiotherapeutic practices. Consecutive patients with intermittent claudication referred for community-based SET were included. Exclusion criteria for SET were pain at rest or tissue loss. All patients received a diagnostic workup consisting of an ankle-brachial index at rest and after exercise. Interventions were exercise therapy according to the guidelines of the Royal Dutch Society for Physiotherapy. The primary outcome measurement was the increase in absolute claudication distance (ACD), assessed using a standardized treadmill protocol by a physiotherapist at baseline and at four, 12, 26, and 52 weeks of SET.

Results: From January 2005 through September 2006, 349 patients were referred by vascular surgeons for community-based SET. A total of 272 patients with intermitten claudication began the program. Of the 349 initially referred patients, 52 could not perform a standard treadmill test but did start community-based SET at a lower level, and 25 patients never started the program. At one year, 129 of the original 272 patients who began community-based SET (47.4\%) were available for analysis of walking distance. In the interim 143 patients discontinued the program for the following reasons: satisfaction with the acquired walking distance $(n=19)$; unsatisfying results $(n=26)$; not motivated ( $n=22)$; (non) vascular intercurrent disease $(n=48)$; and other reasons $(n=28)$. ACD increased significantly from a median of $400 \mathrm{~m}$ at baseline to $1100 \mathrm{~m}$ after 12 months of follow-up $(P<.001)$, corresponding to a median increase of $107.8 \%$.

Conclusion: Community-based SET seems as effective as SET in a hospital-based approach in improving walking distance, however, it has a high dropout rate.

\section{ntroduction}

Intermittent claudication is a common symptom in patients with peripheral arterial disease (PAD). Treatment of patients suffering from intermittent claudication is based on two basic components: treatment of vascular risk factors to prevent future vascular events and treatment of symptoms.' Symptomatic relief of complaints of intermittent claudication can be achieved by exercise therapy, percutaneous transluminal angioplasty (PTA), and surgical revascularization. Exercise therapy is efficacious in improving walking distance ${ }^{2}$ and is considered the first choice of treatment (Fontaine stage II). However, in cases of more proximal disease (e.g., aortic or iliac), primary revascularization is advised.'

The most common exercise therapy prescription consists of one-time oral advice to walk more, usually without supenision or follow-up. ${ }^{3}$ However, the importance of supenvised exercise therapy (SET) is increasingly recognized. ${ }^{1,4} \mathrm{~A}$ recent Cochrane review reported a significant difference in favour of SET compared with an unsupervised exercise program in improving walking distance. ${ }^{5}$ Trials included in this review provided SET programs at a department of physiotherapy or revalidation in a hospital. While this approach is appropriate in trials, there are some limitations in routine clinical practice. First, the capacity of a single department in a hospital is limited and not sufficient to provide SET to all claudication patients in the community. Second, attending at the hospital for two or three times a week is time consuming and expensive for the patient. These piffalls can be solved using a communitybased approach to $\mathrm{SET}_{1}{ }^{6}$ consisting of a selected group of community-based physiotherapists especially trained in applying exercise therapy.

Implementation of community-based SET was first described by Willigendael et al, and the first results in a selected group of patients were promising. ${ }^{8}$ This study describes the results of community-based SET at one year of follow-up compared with clinic-based SET with results known from literature. Furthermore, we give a status report on how community-based SET works in daily practice and describe the possible piffalls of this approach.

\section{Patients and methods}

Study setting

All patients presenting at the vascular outpatient clinic with complaints of intermittent claudication receive a diagnostic workup consisting of an ankle brachial index (ABI)

measurement, which, if below 0.9, was followed by duplex ultrasound of the aortic-liac tract. Patients with occlusive disease of the aortic-iliac tract were referred for diagnostic angiography and PTA or recanalization in the same procedure. Patients with more distal disease were

primarily considered for community-based SET and referred to a physiotherapist participating in the Network for Exercise Therapy Parkstad (NETP)

The NETP provides community-based SET in private physiotherapy practices equally distributed over the region, instead of a department of physiotherapy and rehabilitation in a hospital or rehabilitation clinic. All participating physiotherapists are trained according to the guidelines of the Royal Dutch Society for Physiotherapy (Table 1). ${ }^{9}$ Training of physiotherapists, and the development and implementation of the NETP is described in more detail by Willigendael et al.7

All patients with confirmed PAD received a platelet inhibitor and lipid-lowering medication. Diabetes mellitus and hypertension were treated according to current guidelines. Furthermore, smoking cessation and lifestyle advice were part of a cardiovascular prevention program initiated by a team of vascular nurse practitioners. 
Study population

All consecutive patients referred for community-based SET from January 2005 through September 2006 were included. Patients with complaints of intermittent claudication were eligible for community-based SET after confirmation of PAD with an ABI measurement below 0.9 at rest or decreasing more than 0.15 after exercise. Signs of critical ischemia (e.g., pain in rest, tissue loss) were exclusion criteria for participating in community-based SET.

Exercise program

All participating physiotherapists were participating in the NETP, a community-based program for SET in patients with intermittent claudication. SET was administered according to the guidelines of the Royal Dutch Society for Physiotherapy. ${ }^{9}$

The main goal of SET is to increase absolute claudication distance (ACD), the distance at which severe claudication pain forces cessation of exercise. This goal was achieved by means of interval training with treadmill walking up to (sub)maximal pain. Secondary goals are improving endurance, increasing strength, and correcting walking patterns. Generally, patients started with a frequency of two to three sessions every week of approximately 30 minutes in the first three months. After this initial phase, the frequency was phased down to approximately once every two weeks at six months of follow-up and once every eight weeks at 12 months of follow-up, depending on patient progress and preference. Patients were encouraged to walk on a daily basis to near maximal pain. Furthermore, all participating physiotherapists were instructed to emphasize as much as possible the importance of lifestyle adjustments and smoking cessation.

In The Netherlands, intermittent claudication is one of the chronic diseases that is considered for physiotherapy during the period of one year. Therefore, for every patient, from the tenth session through the period of one year, reimbursement is financed by the National Insurance of Health Care. Reimbursement for the first nine sessions of 30 minutes of exercise therapy depends on the patient's additional and voluntarily health insurance. The minimal advised charge of one session of physiotherapy is 32.07 USD (www.fysionet.nl).

Approximately, the mean costs of one year of community-based SET lie in between 801.75 and 1282.80 USD, dependant on the frequency of the sessions.

Therapy evaluation

Patients were evaluated by their physiotherapist at baseline and at 4, 12, 26, and 52 weeks of follow-up. Patients performed a standardized treadmill test, and their physiotherapists registered walking distances and possible reasons for dropping out of the SET program. A progressive treadmill test was used, with a constant speed of $3.2 \mathrm{~km} / \mathrm{h}$ and an increase in incline of 2\% every two minutes, starting at 0\% incline. ${ }^{10}$ The incline and testing duration were maximized for practical reasons to $10 \%$ and 30 minutes $(1600 \mathrm{~m})$, respectively. Outcomes of these evaluations were registered in a Web-based electronic patient file, available for the physiotherapist as well as the vascular surgeons and nurse practitioners (www.fastguide.eu).

Outcome measurements

The primary outcome measurement was the percentage increase in ACD. Secondary outcome measurements were functional claudication distance (FCD), defined as the distance at which the patient prefers to stop because of claudication pain, and the number of invasive vascular interventions within one year after starting community-based SET.
Table 1: Summary of the Royal Dutch Society for Physical Therapy's guideline on 'Intermittent Claudication'

\begin{tabular}{|c|c|c|}
\hline $\begin{array}{l}\text { Facilities for } \\
\text { SET }\end{array}$ & Equipment & $\begin{array}{l}\text { Treadmill; cycle ergometer; indoor walking space; } \\
\text { small-group } \\
\text { exercise room }\end{array}$ \\
\hline \multirow{3}{*}{$\begin{array}{l}\text { Diagnostic } \\
\text { process }\end{array}$} & $\begin{array}{l}\text { Referral } \\
\text { (general } \\
\text { practitioner / } \\
\text { specialist) }\end{array}$ & $\begin{array}{l}\text { Specific education to provide exercise therapy according to the } \\
\text { recommendations of the guideline is required } \\
\text { Fontaine classification; ankle-brachial index (<0.9); walking } \\
\text { impairment; cardiac risk and capacity; contra-indications }\end{array}$ \\
\hline & $\begin{array}{l}\text { Medical } \\
\text { history } \\
\text { Physical } \\
\text { examination }\end{array}$ & $\begin{array}{l}\text { Disease-specific; co-morbidities; patient-specific complaints } \\
\text { scale } \\
\text { Disease-specific; co-morbidities }\end{array}$ \\
\hline & $\begin{array}{l}\text { Functional } \\
\text { examination }\end{array}$ & Maximum treadmill test; gait and specific function analysis \\
\hline \multirow[t]{4}{*}{$\begin{array}{l}\text { Therapeutic } \\
\text { process }\end{array}$} & Objectives & $\begin{array}{l}\text { Increase maximum walking distance; increase aerobic } \\
\text { endurance; increase pain tolerance and reduce fear of pain; } \\
\text { improve gait } \\
\text { pattern and specific activities (stair climbing, etc); provide } \\
\text { information and induce lifestyle changes }\end{array}$ \\
\hline & $\begin{array}{l}\text { Behavioral } \\
\text { change }\end{array}$ & $\begin{array}{l}\text { Increase pain tolerance; reduce fear of pain; induce active } \\
\text { lifestyle and improve risk factors }\end{array}$ \\
\hline & $\begin{array}{l}\text { Supply } \\
\text { information }\end{array}$ & Disease-specific; exercise therapy; lifestyle \\
\hline & $\begin{array}{l}\text { Therapy } \\
\text { mode and } \\
\text { intensity }\end{array}$ & $\begin{array}{l}\text { Primarily walking up to maximum or submaximum pain; at } \\
\text { least } 6 \text { months; at least } 30 \text { minutes per session; walking } 3 \\
\text { times a week (supervised or homework) }\end{array}$ \\
\hline
\end{tabular}

ACSM denotes American College of Sports Medicine, SET supervised exercise therapy

Furthermore, results were categorized as good, moderate, or unsatisfactory according to an objectively measured increase in ACD and according to the dropout reason of a patient. If patients stated that they were not satisfied with the walking distance at time of dropout, the result was classified as unsatisfactory. If patients stated that the reason of dropout was satisfaction with the regained walking distance, the result was classified as good.

Analysis

All interval and ratio variables are presented as median and inter-quartile-range (IQR) because most variables were not normally distributed. Means are reported to allow comparison of the results of this study with results from the literature. Nominal variables are presented as absolute numbers and percentages.

For every patient, the percentage increase in FCD and ACD compared with baseline was calculated. Exclusion criteria for analysis of walking distances were the absence of 
a baseline measurement or the inability to walk according to the standardized treadmil protocol. All other patients were included in the analysis when data were available. The statistical significance of this within-group difference was analyzed with a Wilcoxon signed-ranks test.

Results were categorized as good, moderate, or unsatisfactory. ${ }^{9}$ A good result was defined as an increase of 100\% or more in ACD or dropping out of SET because of satisfaction with the regained walking distance. A moderate result was defined as an increase in ACD between 50\% and 100\%. The result was defined as unsatisfactory in cases of an ACD increase of less than 50\% or dropping out of SET because of patient dissatisfaction with the acquired walking distance. In patients undergoing a vascular intervention during the year, the last result before the vascular intervention was carried forward. In addition, the last result before dropping out for reasons other than (dis) satisfaction was carried forward.

Statistically significant differences between groups were calculated with a Mann-

Whitney $\mathrm{U}$ test for ordinal and interval variables and a Chi ${ }^{2}$ test for binominal variables. If criteria for a Chi ${ }^{2}$ test were not fulfilled, a Fisher exact test was used. Comparison of more than two groups was performed using a Chi2 test for trend. A value of $\mathrm{P}<.05$ was considered to be statistically significant. Analyses were performed using SPSS 12.0 for Windows (SPSS, Inc, Chicago, III)

\section{Results}

From January 2005 through September 2006, 349 patients with intermittent claudication were referred for communitybased SET Baseline characteristics of this population are summarized in Table 2.

Fifty-two patients were not able to perform a standard treadmill test as described above and were excluded from further analysis. These patients did start community-based SET but at a lower level. Twenty-five patients never started community-based SET for various reasons (Figure 1). These patients were also excluded from further analysis.

A total of 272 patients with intermittent claudication started community-based SET. At one year, 129 patients (47.4\%) were available for analysis of walking distance (Figure 1). The remaining 143 patients discontinued the program for the following reasons: satisfaction with the acquired walking distance $(n=19)$; unsatisfying results $(n=26)$; not motivated $(n=22)$; (non)vascular intercurrent disease $(n=48)$; and other reasons $(n=28)$. No differences in baseline characteristics were found between withdrawals and the population that completed 12 months of community-based SET, with the exception of the greater number of current smokers $(\mathrm{P}=.046)$ in the withdrawal group (Table 2).

\section{Walking distances}

The median FCD and ACD at baseline were $230.0 \mathrm{~m}$ (mean, $325.0 \mathrm{~m}$ ) and $400.0 \mathrm{~m}$ (mean, $493.1 \mathrm{~m}$ ), respectively. After 12 months of follow-up, the median walking distances significantly increased to $700.0 \mathrm{~m}$ (mean, $867.0 \mathrm{~m}$ ) for FCD and $1100.0 \mathrm{~m}$ (mean, 1053.5 $\mathrm{m})$ for $\mathrm{ACD}(\mathrm{P}<001)$. This increase corresponds to a median increase of 166.7\% (mean 279.3\%) and 107.8\% (mean, 196.9\%) compared with baseline for FCD and ACD, respectively. Notably, most of the increase in walking distance occurred in the first six months of community-based SET. No further improvement in walking distance was seen between six and 12 months of follow-up. Table 3 shows more details about the different walking distances at 4, 12, and 26 weeks of follow-up.
Course of response to SET

The results of the total cohort $(n=272)$ were categorized as good, moderate, or unsatisfactory. Because of the chosen definitions, 37 patients could not be classified. Of these, 33 dropped out before the first follow-up measurement, and four patients had a vascular intervention before four weeks of follow-up, resulting in 235 patients who were available for analysis. The clinical course of the cohort is graphically represented in Figure 2, in which the size of the arrows reflects the number of patients. As Figure 2 shows, after 52 weeks of follow-up or at the moment a patient dropped out, 112 patients (47.7\%) showed a good result, 38 (16.2\%) had a moderate result, and 85 (36.2\%) had an unsatisfactory result. Good or unsatisfactory results achieved at 12 weeks of follow-up were likely to persist at 26 and 52 weeks of follow-up.

\section{Vascular interventions}

Out of 272 patients, 43 had a vascular intervention within the follow-up period of one year, of whom 33 received a PTA, six had bypass surgery, and four had other vascular interventions. In 39 patients (90.7\%), the indication was intermittent claudication, and in four patients $(9.3 \%)$, it was critical limb ischemia.

The distribution of the interventions in the different result categories showed a non-significant trend in those with an unsatisfactory result. The frequency of interventions in the group showing good results was $2.7 \%$. In the groups showing moderate and unsatisfactory results, these percentages were $13.2 \%$ and $25.9 \%$, respectively.

\section{Additional observations}

Fifty-two patients were not able to walk at the speed of $3.2 \mathrm{~km} / \mathrm{h}$ of the standardized treadmill protocol. In general, these patients had poorer clinical characteristics; they were older $(P<.001)$, had diabetes more often $(P=.014)$, and had a significantly lower resting $\mathrm{ABI}(\mathrm{P}=.008)$ than patients starting the usual community-based SET. However, these 52 patients started community-based SET at their own level, with varying speeds and inclines during treadmill testing. Another striking difference between the group starting community-based SET at a low level and the group starting the usual SET was the dropout percentage. In the low level group, the dropout rate was $84.6 \%$ compared with $52.5 \%$ in the standard SET group $(P<.001)$. Seven patients from the lower-level group had a vascular intervention, all for intermittent claudication; four of these patients had PTA, and three had bypass surgery. This outcome was not significantly different from the standard SET group.

Twenty-five patients never started community-based SET for different reasons (Figure 1). Within one year after referral, three patients received a PTA and one patient received another vascular intervention. This outcome was not significantly different from the number of interventions in the group starting community-based SET. Patients never starting community-based SET were more likely to be men $(P=.012)$ compared with patients who did start SET.

There were no other significant differences between these two groups

\section{Discussion}

In this study, we have shown that one year of community-based SET results in highly significant increases in FCD as well as ACD after 4, 12, 26, and 52 weeks of follow-up. However, community-based SET has a high dropout rate. These results for walking distance as well as for dropout are in line with the results after six months of community-based SET published recently by Bendermacher et al. ${ }^{8}$ 


\begin{tabular}{|c|c|c|c|c|}
\hline Characteristic & $\begin{array}{l}\text { Total population, } \\
\mathrm{n}=349\end{array}$ & $\begin{array}{l}\text { Population } \\
\text { completing } \\
12 \text { montWhs, } \\
n=137\end{array}$ & $\begin{array}{l}\text { Withdrawals, } \\
n=212\end{array}$ & $P$ value \\
\hline Age (year) & $66.0(58.6-74.0)$ & $66.4(58.6-73.7)$ & $65.7(58.5-74.3)$ & NS \\
\hline Men & $219 / 349(62.8 \%)$ & $88 / 137(64.2 \%)$ & $131 / 212(61.8 \%)$ & NS \\
\hline BMI & $\begin{array}{l}26.1(23.6-29.0) \\
n=331\end{array}$ & $\begin{array}{l}26.6(23.9-29.4) \\
n=136\end{array}$ & $\begin{array}{l}25.9(23.1-28.9) \\
n=195\end{array}$ & NS \\
\hline Resting ABI & $\begin{array}{l}0.70(0.60-0.88) \\
n=342\end{array}$ & $\begin{array}{l}0.71(0.60-0.88) \\
n=135\end{array}$ & $\begin{array}{l}0.70(0.60-0.88) \\
n=207\end{array}$ & NS \\
\hline Current smokers & 168/341 (49.3\%) & $58 / 136(42.6 \%)$ & $110 / 205(53.7 \%)$ & .046 \\
\hline Hypertension & 266/349 (76.2) & $103 / 137(75.2 \%)$ & $163 / 212(76.9 \%)$ & NS \\
\hline Diabetes mellitus & $117 / 349(33,5 \%)$ & 48/137 (35.0\%) & $69 / 212(32.5 \%)$ & NS \\
\hline Hypercholesterolemia & 267/349 (76.5\%) & 109/137 (79.6\%) & 158/212 (75.4\%) & NS \\
\hline Coronary heart disease & 93/349 (26.6\%) & 35/137 (25.5\%) & $58 / 212(27.4 \%)$ & NS \\
\hline Cerebrovascular disease & 47/349 (13.5\%) & 13/137 (9.5\%) & $34 / 212(16.0 \%)$ & NS \\
\hline COPD & 50/349 (14.3\%) & $22 / 137(16.1 \%)$ & 28/212 (13.2\%) & NS \\
\hline Arthrosis & 19/349 (5.4\%) & $7 / 137(5.1 \%)$ & $12 / 212(5.7 \%)$ & NS \\
\hline $\begin{array}{l}\text { Previous vascular } \\
\text { intervention }\end{array}$ & $113 / 349(32.3 \%)$ & 45/137 (32.8\%) & $68(32.1 \%)$ & NS \\
\hline $\begin{array}{l}\text { Systolic blood pressure } \\
(\mathrm{mmHg})\end{array}$ & $\begin{array}{l}150.0(135.0-170.0) \\
n=344\end{array}$ & $\begin{array}{l}150.0(135.0-172.3) \\
n=136\end{array}$ & $\begin{array}{l}150.0(132.3-170.0) \\
n=208\end{array}$ & NS \\
\hline Glucose (mmol/l) & $\begin{array}{l}5.9(5.3-7.1) \\
n=330\end{array}$ & $\begin{array}{l}5.9(5.2-7.1) \\
n=127\end{array}$ & $\begin{array}{l}5.9(5.3-7.0) \\
n=203\end{array}$ & NS \\
\hline $\mathrm{HbA1C}(\%)$ & $\begin{array}{l}6.0(5.8-7.1) \\
n=238\end{array}$ & $\begin{array}{l}6.2(5.8-7.2) \\
n=94\end{array}$ & $\begin{array}{l}6.0(5.7-6.9) \\
n=144\end{array}$ & NS \\
\hline Cholesterol (mmol/l) & $\begin{array}{l}5.2(4.2-6.0) \\
n=333\end{array}$ & $\begin{array}{l}5.1(4.3-6.0) \\
n=130\end{array}$ & $\begin{array}{l}5.2(4.1-5.9) \\
n=203\end{array}$ & NS \\
\hline $\mathrm{HDL}(\mathrm{mmol} / \mathrm{l})$ & $\begin{array}{l}1.33(1.12-1.60) \\
n=316\end{array}$ & $\begin{array}{l}1.35(1.12-1.67) \\
n=123\end{array}$ & $\begin{array}{l}1.31(1.09-1.58) \\
n=193\end{array}$ & NS \\
\hline LDL (mmol/l) & $\begin{array}{l}2.8(2.0-3.5) \\
n=304\end{array}$ & $\begin{array}{l}2.6(2.1-3.5) \\
n=119\end{array}$ & $\begin{array}{l}2.9(2.0-3.6) \\
n=185\end{array}$ & NS \\
\hline Triglycerides (mmol/l) & $\begin{array}{l}1.60(1.11-2.22) \\
n=311\end{array}$ & $\begin{array}{l}1.52(1.08-2.42) \\
n=122\end{array}$ & $\begin{array}{l}1.67(1.13-2.22) \\
n=189\end{array}$ & NS \\
\hline $\mathrm{FCD}(\mathrm{m})$ & $\begin{array}{l}230.0(120.0- \\
427.5) \\
n=269\end{array}$ & $\begin{array}{l}260.0(130.0- \\
450.0) \\
n=127\end{array}$ & $\begin{array}{l}205.0(117.0-382.5) \\
n=142\end{array}$ & NS \\
\hline $\mathrm{ACD}(\mathrm{m})$ & $\begin{array}{l}400.0(323.0- \\
630.8) \\
n=272\end{array}$ & $\begin{array}{l}276.0(450.0- \\
689.0) \\
n=129\end{array}$ & $\begin{array}{l}370.0(207.0-590.0) \\
n=143\end{array}$ & NS \\
\hline
\end{tabular}

NS indicates not significant, BMI body mass index, ABI ankle brachial index, COPD chronic obstructive pulmonary disease, FCD functional claudication distance, and ACD absolute claudication distance.
Figure 1: Flow chart of the total population referred for community-based supervised exercise therapy.

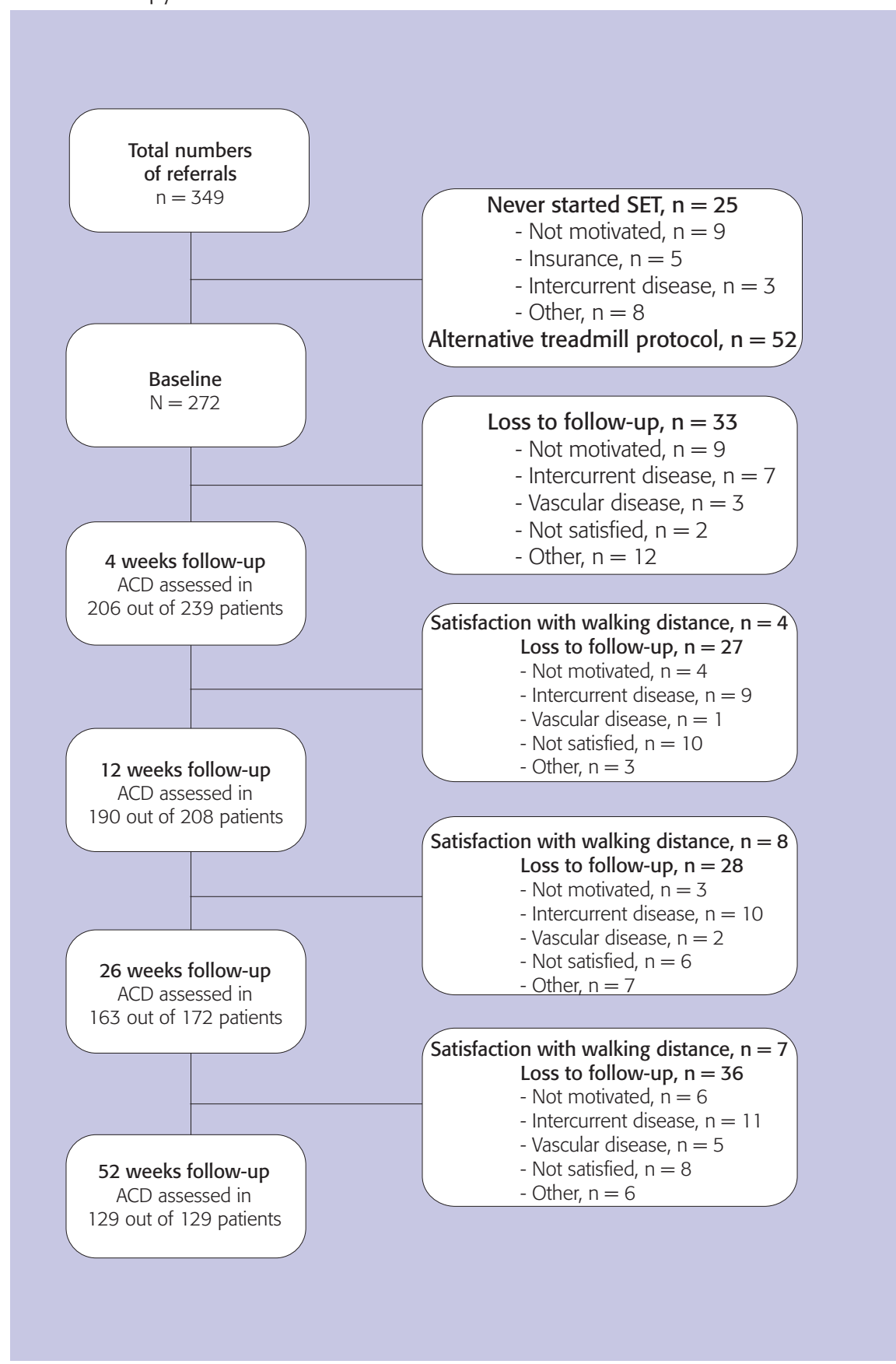

ACD: Absolute claudication distance. 


\begin{tabular}{|c|c|c|c|c|}
\hline \multicolumn{5}{|c|}{ Median increase functional claudication distance* } \\
\hline Follow up & N & $\begin{array}{l}\text { Median (IQR) } \\
\text { metres }\end{array}$ & $\begin{array}{l}\text { Absolute median increase } \\
\text { (IQR) metres }\end{array}$ & $\begin{array}{l}\text { Median \% increase } \\
(\text { IQR) }\end{array}$ \\
\hline Baseline & 269 & $230.0(120.0-427.5)$ & - & - \\
\hline 1 month & 201 & $412.0(275.0-630.0)$ & $110.0(30.0-245.0)$ & $47.7 \%(8.3-115.6)$ \\
\hline 3 months & 187 & $510.0(324.0-840.0)$ & $220.0(90.0-430.0)$ & $100.0 \%(34.5-260.9)$ \\
\hline 6 months & 159 & $650.0(350.0-1200.0)$ & $340.0(143.0-747.0)$ & $140.0 \%(59.1-380.0)$ \\
\hline 12 months & 125 & $700.0(395.0-1600.0)$ & $440.0(172.0-905.0)$ & $166.7 \%(79.4-342.2)$ \\
\hline \multicolumn{5}{|c|}{ Median increase absolute claudication distance } \\
\hline Follow up & N & $\begin{array}{l}\text { Median (IQR) } \\
\text { metres }\end{array}$ & $\begin{array}{l}\text { Absolute median increase } \\
\text { (IQR) metres }\end{array}$ & $\begin{array}{l}\text { Median \% increase } \\
(\text { IQR) }\end{array}$ \\
\hline Baseline & 272 & $400.0(232.0-630.8)$ & - & - \\
\hline 1 month & 206 & 618.5 (407.5-985.0) & $140.0(40.0-330.0)$ & $37.1 \%(10.5-77.6)$ \\
\hline 3 months & 190 & $792.0(487.5-1242.5)$ & $265.0(110.0-592.5)$ & $79.4 \%(33.9-169.0)$ \\
\hline 6 months & 163 & 900.0 (490.0-1600.0) & $360.0(149.0-850.0)$ & 87.5\% (33.3-229.2) \\
\hline 12 months & 129 & $1100.0(520.0-1600.0)$ & $506.0(185.5-890.0)$ & $107.8 \%(47.8-201.9)$ \\
\hline
\end{tabular}

IQR indicates interquartile range.

*In some patients, only absolute claudication distance was measured.

\begin{tabular}{lllll|}
\hline \multicolumn{5}{l}{ Table 4: Increase in absolute claudication distance / time in prior studies using clinic-based SET } \\
\hline Trial & Patients & $\begin{array}{l}\text { Duration SET } \\
\text { (months) }\end{array}$ & $\begin{array}{l}\text { Increase in ACD / ACT } \\
(\%)\end{array}$ \\
& & & 6 months & 12 months \\
Creasy12 & 16 & 12 & $110 \%$ & $110 \%$ \\
Dahllof13 & 10 & 6 & $116 \%$ & - \\
Lundgren 15 & 23 & $6-12$ & - & $151 \%$ \\
Mannario 16 & 10 & 6 & $86 \%$ & - \\
Tisi18 & 22 & 1 & $60 \%$ & $68 \%$ \\
Cheetham11 & 28 & 6 & $129 \%$ & - \\
Degischer 14 & 19 & 3 & $70 \%$ & - \\
Kakkos 17 & 8 & 6 & $52 \%$ & - \\
Savage20 & 11 & 6 & $42 \%$ & $109 \%$ \\
Patterson 19 & 25 & 3 & $159 \%$ & $92 \%$ \\
Mean \% increase overall & & & $92 \%$ & - \\
\hline
\end{tabular}

Figure 2: Clinical course of the total cohort.

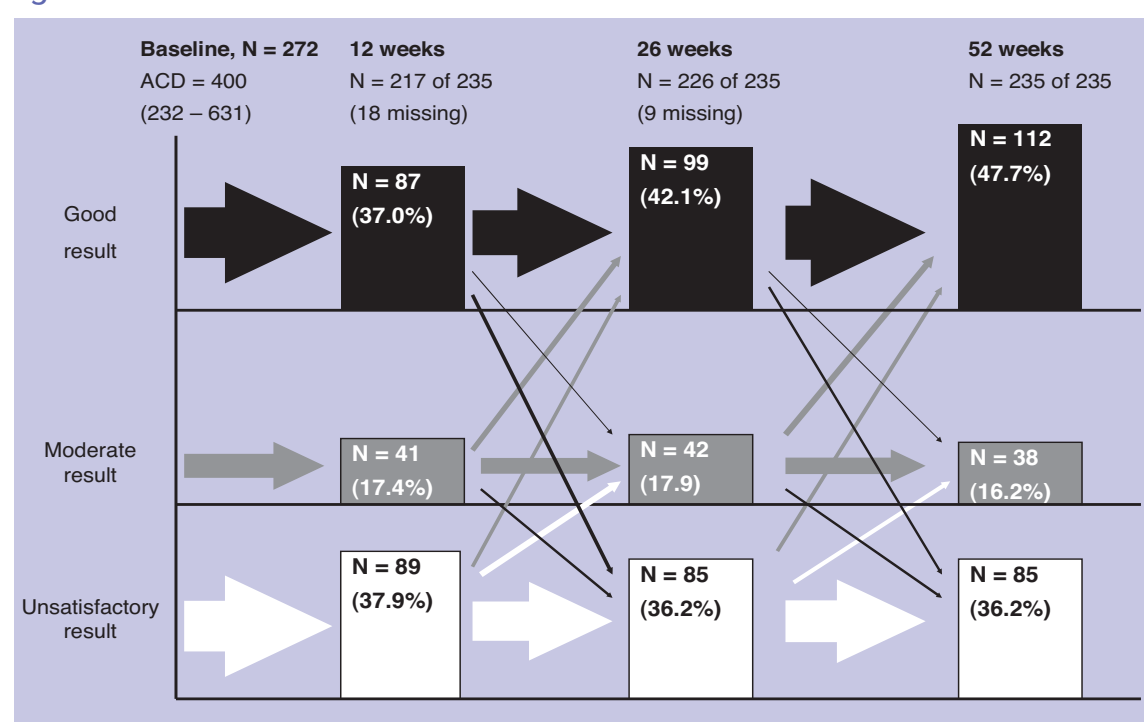

The arrows represent movement of patients between different result categories in time and the blocks indicate the results at the different follow-up measurements. The size of the arrows and blocks reflect the number of patients. Black, grey, and white represent patients with a good, moderate, and unsatisfactory result respectively. Good result: increase in ACD of $\geq 100 \%$ or patient satisfaction with the walking distance. Moderate result: increase in ACD $>50 \%$ and $<100 \%$. Unsatisfactory result: increase in $A C D<50 \%$ or patient dissatisfaction with the walking distance. $A C D$ : Absolute claudication distance.

Most reports on SET for patients with intermittent claudication are situated in an outpatient clinical setting, but these also show comparable results in improving walking distance. ${ }^{2,5,1-20}$ Table 4 gives an overview of results of clinic-based SET after 6 and

12 months of follow up., ${ }^{2,5}$ The overall mean percentages increase of clinic-based SE after 6 and 12 months of follow up are 92\% and 108\%, respectively. In our study, we found median increases in ACD of 88\% (mean, 191\%) and 108\% (mean, 197\%) after 6 and 12 months of follow up, suggesting community-based SET is as least equal to clinic-based SET.

Furthermore, an interesting observation is that a good or unsatisfactory result achieved after three months of community-based SET was unlikely to have changed at 6 and 12 months. This finding could have consequences for clinical care and could lead to the notion that if results are unsatisfactory at 3 months of community-based SET, other treatment options should be considered. Naturally, at that time, severity of complaints and the risk of invasive interventions must be taken into account.

Patients referred for community-based SET are offered one year of exercise therapy,

based on limited reimbursement up to one year. However, after 6 months of SET, walking distance had stabilized and FCD and ACD did not increase further. The reason for this phenomenon is unclear. A possible explanation is that after 6 months of community-based SET, patients achieve their maximum, entering into a plateau phase. Another explanation could be that within a year of community-based SET, the frequency of sessions gradually decreases, leading to maintenance of but not increases in walking distance. In a metaanalysis by Gardner et al., the best duration of a SET program was six months or longer, but 
no distinction was made between six and 12 months. ${ }^{21}$ Dose-response research is needed to address this issue.

Another issue of discussion is the mode of exercise. Currently, the recommended mode of exercise mainly consists of treadmill walking to near maximal pain, according to the guidelines of the Royal Dutch Society for Physiotherapy. ${ }^{9}$ However, recent research suggests that a pain-free mode of training also has significant effects on walking distance compared with a non-exercising control group ${ }^{22,23}$ Possible advantages of pain-free training could be better compliance and lower dropout rates. To determine if pain-free walking is as effective as walking to near maximal pain, further research is needed.

Comparing non-supervised exercise therapy with SET, the results with SET are statistically significantly better, leading to approximately 150-m and 225-m greater increases in ACD after three months ${ }^{5}$ and six month $\mathrm{s}^{24}$ of follow-up, respectively. Ideally, according to the TransAtlantic Inter-Society Consensus Document on management of PAD (TASC-II), SET should be made available for all patients with intermittent claudication.' Implementing SET for intermittent claudication in an outpatient clinic, however, is very complicated or maybe even impossible, based on its disadvantages. First of all, the capacity of the usual department of rehabilitation and physiotherapy in a hospital is not sufficient for the numbers of patients with intermittent claudication who are eligible for non-invasive therapy. Furthermore, patients are often limited in their transport to the hospital in terms of costs and time. Community-based SET is an easy and good alternative for structurally implementing SET for every patient with intermittent claudication.

In addition to the effects on walking distance, SET could have a beneficial effect related to vascular risk factor management. Exercise has a known positive influence on vascular risk factors such as hypertension, hypercholesterolemia, and diabetes mellitus. ${ }^{25}$ Furthermore, reports show that patients with $\mathrm{PAD}^{26}$ (or more specifically, intermittent claudication ${ }^{27}$ who are physically active are less likely to die compared with a group of sedentary patients with PAD, 26,27 After adjustments for age, ABI, and body mass index, these results are similar in patients with intermittent claudication. ${ }^{27}$

A possible disadvantage of community-based SET could be the large number of participating physiotherapists, leading to a lower volume of patients and less experience per physiotherapist. Thus, by referring patients only to specifically trained physiotherapists and not to just any physiotherapist, this problem can be addressed. Furthermore, by organizing SET in a community-based setting, an increasing number of patients with intermittent claudication can be treated, leading to greater numbers of experienced physiotherapists. In addition, regular refresher courses with all participating physiotherapists can ensure adequate and shared knowledge. However, the number of referred patients, and thus the experience of the physiotherapists, is dependant on the utilization of the program.

The high number of dropouts (40.0\% within six months and 52.6\% within 12 months) is a disadvantage of community-based SET. However, in the literature, we find corresponding dropout percentages after six months of SET, from 10\% up to $50 \% .^{17,22,28-30}$ Only two studies report a dropout percentage after 12 months, ranging from $16 \%$ to $41 \% .15,31$ It should be realized that in the literature, dropout is observed in randomized controlled trials that have a highly selected patient population with strictly chosen inclusion and exclusion criteria. Presumably, dropout rates of community-based SET in usual practice seem not to be increased compared with clinic-based SET.
Although the presence of intermittent claudication leads to functional impairment for the patient, the clinical course as far as the leg is concerned is surprisingly stable in most cases. ${ }^{1}$ For this reason, it is usually acknowledged that invasive interventions with known morbidity and mortality should be used with restraint. The main reason for patients to stop community-based SET is intercurrent disease, and not unsatisfactory results. Thus, if a patient stops SET, the reason should be evaluated. The physician can use this moment to evaluate to the next level of decision making and consider an invasive intervention, if clinically indicated.

Our data suggest that after three months of SET, a physician can properly evaluate a patient's response to SET. In summary, SET represents a viable treatment for intermittent claudication with no known complications and has the potential to treat patients safely, preventing an invasive vascular intervention in a number of patients. Although many patients discontinue SET prematurely, its noninvasive nature and satisfactory improvement in the majority of patients are reasons to use it as the initial treatment in patients with intermittent claudication.

In case of unsatisfactory results, the option for PTA or surgical revascularization is still open if clinically indicated.

\section{Conclusion}

Community-based SET seems as efficacious as SET in a clinical study approach in improving walking distance in patients with intermittent claudication, however, has high dropout rates. Furthermore, good and unsatisfactory results after three months are likely to persist, which could be useful for a physician in evaluating response to community-based SET and planning further treatment.

\section{Acknowledgements}

First, we would like to thank all the participating physiotherapists of the Network for Exercise Therapy Parkstad for their effort and training patients. Furthermore, we would like to thank the members of the Dutch Platform for Peripheral Arterial Disease for their contribution and new ideas for analysis and discussion. Finally, we would like to thank Prof. R.A. de Bie for use of the Web-based electronic patient files. 


\section{References}

1. Norgren L, Hiatt WR, Dormandy JA, Nehler MR, Harris KA, Fowkes FG. Inter-Society Consensus for the Management of Peripheral Arterial Disease (TASC II). J Vasc Surg 2007;45 Suppl S:S5-67.

2. Leng CC, Fowler B, Enst E. Exercise for intermittent claudication. Cochrane Database Syst Rev 2000(2):CD000990

3. Bartelink ML, Stoffers HE, Biesheuvel CJ, Hoes AW. Walking exercise in patients with intermittent claudication. Experience in routine clinical practice. Br J Gen Pract 2004;54:196-200.

4. Stewart AH, Lamont PM. Exercise for intermittent claudication. Supervised programmes should be universally available. BMJ 2001;323: 703-4.

5. Bendermacher BL, Willigendael EM, Teijink JA, Prins MH. Supervised exercise therapy versus non-supervised exercise therapy for intermittent claudication. Cochrane Database Syst Rev 2006(2):CD005263

6. Bendermacher BL, Kruidenier LM, Teijink JA Supenised exercise therapy for intermittent claudication in a community-based setting. In: Greenhalgh RM, editor. More vascular and endovascular challenges. 1st ed. London: BIBA Medical Ltd; 2007. p. 305-15.

7. Willigendael EM, Bendermacher BL, van der Berg C, Welten RJ, Prins MH, Bie de RA, et al. The development and implementation of a regiona network of physiotherapists for exercise therapy in patients with peripheral arterial disease, a preliminary report. BMC Health Serv Res 2005;5:49.

8. Bendermacher BL, Willigendael EM, Nicolai SP, Kruidenier LM, Welten RJ, Hendriks E, et al. Supenised exercise therapy for intermittent claudication in a community-based setting is as effective as clinicbased. J Vasc Surg 2007;45:1 192-6.

9. Jongert MWA, Hendriks HJM, Van Hoek J, KlaasboerKogelman K, Robeer GG, Simens B, et al. KNGFrichtlinn Claudicatio Intermittens. Ned Tijdschr Fysiother 2003;6 (Suppl):3-58.
10. Gardner AW, Skinner JS, Cantwell BW, Smith LK. Progressive vs single-stage treadmill tests for evaluation of claudication. Med Sci Sports Exerc 1991;23:402-8.

11. Cheetham DR, Burgess L, Ellis M, Williams A, Greenhalgh RM, Davies AH. Does supervised exercise offer adjuvant benefit over exercise advice alone for the treatment of intermittent claudication? A randomised trial Eur J Vasc Endovasc Surg 2004:27:17-23.

12. Creasy TS, McMillan PJ, Fletcher EW, Collin J, Morris PJ. Is percutaneous transluminal angioplasty better than exercise for claudication? Preliminary results from a prospective randomised trial. Eur J Vasc Surg 1990;4:135-40.

13. Dahllof AG, Bjorntorp P, Holm J, Schersten T. Metabolic activity of skeletal muscle in patients with peripheral arterial insufficiency. Eur J Clin Invest 1974;4:9-15.

14. Degischer S, Labs KH, Hochstrasser J, Aschwanden M, Tschoepl M, Jaeger KA.

Physical training for intermittent claudication: comparison of structured rehabilitation versus home-based training. Vasc Med 2002;7:109-15.

15. Lundgren F, Dahlllof AG, Lundholm K, Schersten T, Volkmann R. Intermittent claudication-surgical reconstruction or physical training? A prospective randomized trial of treatment efficiency. Ann Surg 1989; 209:346-55.

16. Mannarino E, Pasqualini L, Innocente S, Scricciolo V, Rignanese A, Ciuffetti G. Physical training and antiplatelet treatment in stage II peripheral arterial occlusive disease: alone or combined? Angiology 1991;42:513-21.

17. Kakkos SK, Geroulakos G, Nicolaides AN Improvement of the walking ability in intermitten claudication due to superficial femoral artery occlusion with supervised exercise and pneumatic foot and calf compression: a randomised controlled trial. Eur J Vasc Endovasc Surg 2005;30:164-75.
18. Tisi PV, Hulse M, Chulakadabba A, Gosling P, Shearman CP. Exercise training for intermitten claudication: does it adversely affect biochemical markers of the exercise-induced inflammatory response? Eur I Vasc Endovasc Surg 1997; 14:344-50.

19. Patterson RB, Pinto B, Marcus B, Colucci A, Braun $T$, Roberts M. Value of a supenised exercise program for the therapy of arterial claudication. J Vasc Surg 1997;25:312-8; discussion 8-9. 20 Savage P, Ricci MA, Lynn M, Gardner A, Knight S, Brochu $M$, et al. Effects of home versus superised exercise for patients with intermittent claudication. J Cardiopulm Rehabil 2001:21:152-7.

20.Savage P, Ricci MA, Lynn M, Gardner A, Knight S, Brochu $M$, et al. Effects of home versus supenised exercise for patients with intermittent claudication. Cardiopulm Rehabil 2001:21:152-7.

21.Gardner AW, Poehlman ET. Exercise rehabilitation programs for the treatment of claudication pain. A meta-analysis. JAMA 1995;274: 975-80.

22. Mika P, Spodaryk K, Cencora A, Mika A. Red blood cell deformability in patients with claudication after pain-free treadmill training. Cli J Sport Med. 2006;16(4):335-340.

23. Mika P, Spodank K, Cencora A, Unnithan VB, Mika A. Experimental model of pain-free treadmill training in patients with claudication. Am J Phys Med Rehabil 2005;84:756-62.

24. Kruidenier $L M$, Bendermacher BL, Willigendae EM, Teijink JA, Prins MH. [From the Cochrane Library: increased walking distance through supenvised exercise therapy in patients with intermittent claudication]. Ned Tijdschr Geneeskd 2008;152:321-3.

25.Shephard RJ, Balady GJ. Exercise as cardiovascular therapy. Circulation 1999;99:963-2.
26. Garg PK, Tian L, Criqui MH, Liu K, Ferrucci L, Guralnik JM, et al. Physical activity during daily life and mortality in patients with peripheral arterial disease. Circulation 2006; 1 14:242-8.

27. Gardner AW, Montgomery PS, Parker DE. Physica activity is a predictor of all-cause mortality in patients with intermittent claudication.J Vasc Surg 2008;47:117-22

28. Regensteiner JG, Steiner JF, Hiatt WR. Exercise training improves functional status in patients with peripheral arterial disease. J Vasc Surg 1996;23:104-15.

29. Gardner AW, Katzel LI, Sorkin JD, Bracham DD, Hochberg MC, Flinn WR, et al. Exercis rehabilitation improves functional outcomes and peripheral circulation in patients with intermitten claudication: a randomized controlled trial. J Am Geriatr Soc 2001;49:755-62.

30. Collins EG, Langbein WE, Orebaugh C, Bammer C, Hanson K, Reda D, et al. Cardiovascular training effect associated with polestriding exercise in patients with peripheral arterial disease. J Cardiovasc Nurs 2005:20:177-85.

31. Gelin J, Jivegard L, Taft C, Karlsson J, Sullivan M, Dahllof AG, et al. Treatment efficacy of intermittent claudication by surgical intervention, supervised physical exercise training compared to no treatment in unselected randomised patients. I: one year results of functional and physiological improvements. Eur $J$ Vasc Endovasc Surg 2001;22: 107-13 


\section{CHAPTER 9}

Muliticenter

randomized

clinical trial

of supervised

exercise therapy

with or without

feedback versus

walking advice

for intermittent

claudication

Saskia P.A. Nicolai

Joep A.W. Teijink

Edith M. Willigendael

Bianca L.W. Bendermacher

Lotte M. Kruidenier

Manuela Joore

Rob J. van Det

Paul J.G. Jörning

Anco Vahl

Rudolph P. Tutein Nolthenius

Philippe W.M. Cuypers

Marie-Louise E.L. Bartelink

Erik J.M. Hendriks

Rob A. de Bie

Martin H. Prins

Submitted 


\section{Abstract}

Background: The initial treatment for intermittent claudication is supervised exercise therapy (SET). Due to limited capacity and transport costs of clinic based SET, a concept of SET provided by local physiotherapists was developed. We hypothesized that provision of daily feedback with an accelerometer in addition to SET would further increase walking distance.

Methods: This multicenter randomized trial compared exercise therapy in the form of a 'go home and walk' advice (WA), SET, and SET with feedback in patients with intermittent claudication. Primary outcome was the change in absolute claudication distance and secondary outcomes were the change in functional claudication distance, walking impairment questionnaire (WIQ) and Short Form 36 (SF-36) after 12 months.

Results: In 11 centres 102, 109, and 93 patients were included in the WA, SET, and SET with feedback groups. Of these, 83, 93, and 76 respectively, could be analysed. The median change in walking distance between 12 months and baseline was 110 (interquartile range (IQR) 0 to 300) meters in the WA-group, 310 (IQR 145 to 995) meters in the SET-group, and 360 (IQR 173 to 697) meters in the SET with feedback group ( $P<0.001$ WA versus SET). WIQ scores and relevant domains of the SF-36 improved statistically significantly in the SET-groups.

Conclusion: SET is more effective than WA in improving walking distance, WIQ scores, and quality of life for patients with intermittent claudication. Additional feedback with an accelerometer did not result in further improvement. SET programs should be made available for all patients with intermittent claudication.

\section{Introduction}

Atherosclerotic disease of the arteries of the lower extremities resulting in walking impairment, typically described as muscular leg pain during exercise and relieved by rest, is defined as intermittent claudication. According to (inter)national guidelines the initial treatment of patients with intermittent claudication is (supervised) exercise therapy combined with cardiovascular risk management., 2

The beneficial effect of exercise therapy is well known. Various exercise programs have been shown to improve maximal walking distance by $150 \% .{ }^{3}$ Exercise therapy is often prescribed in the form of a 'go home and walk' advice. However, compliance with this strategy is known to be low. ${ }^{4}$ A Cochrane review demonstrated a benefit of supervised exercise therapy (SET) over non-supenvised exercise programs. ${ }^{5}$ In the majority of the reviewed small studies SET was offered in a clinical setting, either in an outpatient clinic for physiotherapy and rehabilitation or in a vascular laboratory. Clinic-based patient care has several disadvantages, including limited capacity of the institution and high transportation cost for the individual patient. For this reason, a network of physiotherapists providing SET, in proximity to the patient's home was developed. ${ }^{6}$ The first results suggested that SET provided by local physiotherapists could be at least as effective as SET in a clinic-based setting.7.8

Although SET offers the benefit of adequate (weekly) coaching, the overall superior effect of SET is likely partially due to improved compliance to the exercise regime. We hypothesized that the use of an accelerometer, which provides daily therapy feedback, with SET affords more effective coaching and may result in a further increase in walking distance. Hence, a multicenter randomized clinical trial was conducted to compare exercise therapy in the form of a 'go home and walk' advice, as is still common practice, with SET provided by local physiotherapists with or without daily therapy feedback.

\section{Methods}

Patients

Eleven outpatient vascular surgery clinics distributed over the Netherlands participated in the Exercise Therapy in Peripheral Arterial Disease (EXITPAD) study.

Patients with peripheral arterial disease, stage II according to Fontaine, who were considered for conservative treatment, were eligible. Inclusion criteria were an anklebrachial index (ABI) below 0.9 and an absolute claudication distance (ACD) of less then 500 metres as assessed with a standardized treadmill test. Exclusion criteria included prior exercise therapy, previous peripheral vascular intervention, insufficient command of the Dutch language, serious cardiopulmonary limitations (NYHA-3-4), previous lower-limb amputation, psychiatric instability, and other serious co-morbidity which may hinder physical training.

Eligible patients were asked to participate and had to provide written informed consent. The study was approved by the institutional review board of the Atrium medical center and at each participating site and registered at clinical trials.gov (NCT00279994). For this study a grant from The Netherlands Organisation for Health Research and Development (ZonMw), a non-profit organisation was obtained.

Randomization and blinding

Patients were randomized to exercise therapy in the form of a walking advice (WA) only, SET provided by local (community based) physiotherapists or SET provided by local physiotherapists with the additional use of an accelerometer to provide daily feedback. Randomization took place centrally by telephone, and numbers were generated by a computer generated block randomization list (block size 9, first 
block opened at random) stratified per center. The local vascular surgeons enrolling patients, the patients, and their physiotherapists if applicable, were, inherent to the study design, not blinded to group assignment. However, study personnel performing treadmill tests and collecting questionnaires, were blinded for previous outcomes and group assignment. Patients were instructed to refrain from commenting on treatment assignment and therapy progress during the assessments.

Interventions

Patients randomised to the WA-group received an oral walking advice, together with a brochure distributed by the Patients Association of Vascular Diseases explaining exercise therapy. ${ }^{9}$ Patients were instructed by their attending vascular surgeons (who enrolled them in the study) to complete three training sessions per day. During each session, maximal pain level should be reached three times.

Patients randomized to the SET-groups were referred to a local physiotherapist.

For each participating center a network of local physiotherapists was trained in SET according to Royal Dutch Society for Physical Therapy and the Dutch Institute of Allied Health Care. ${ }^{6}$ An average network consists of 20 to 30 physiotherapists equally distributed over the region of the hospital. The educational program ensured that all patients received SET according to the guidelines of the Royal Dutch Society for Physical Therapy. ${ }^{10}$ The main goal was to increase patient's walking distance by interval training up to sub-maximal pain with short walking intervals. Additionally, the program consisted of walking pattern improvement and enhancement of endurance and strength. Generally, patients started with a frequency of 2 to 3 sessions of 30 minutes a week. This was tailored to the individual need of the patient during the treatment year. Furthermore, all SET patients were encouraged to walk as much as possible. Patients assigned to SET with feedback additionally received an accelerometer (Personal Activity Monitor (PAM), PAM B.V., Doorwerth, The Netherlands).11, 12 The PAM is a performance based accelerometer to assess physical activity during normal life. The PAM produces a cumulative score which is continuously displayed for feedback. The patient was instructed to wear this instrument continuously during the day for the period of one year. The PAM scores were recorded by the patient every day. Based on the PAM scores, physiotherapists gave feedback to patients regarding walking efforts outside the SET program.

The manufacturer (PAM B.V.) provided us with technical support. PAM B.V. did not

have access to outcome data and did not participate in data analysis or preparation of the manuscript.

\section{Outcome measurements}

The primary outcome measurement was the change in absolute claudication distance (ACD). Secondary outcome measurements were the change in functional claudication distance (FCD), the Walking Impairment Questionnaire (WIQ), and the Short Form 36 (SF-36). After 3, 6, 9 and 12 months of follow-up treadmill assessments were performed and the WIQ and SF-36 were filled-out.

The ACD was defined as the moment the patient had to stop walking due to a maximal pain level. The functional claudication distance (FCD) was defined as the moment the patient preferred to stop walking due to the pain. Treadmill testing is the most commonly used quantitative measure to assess walking ability. ${ }^{1}{ }^{A C D}{ }^{13}$ and $\mathrm{FCD}^{14}$ are both reliable tools to evaluate walking distance in patients with intermittent claudication. Walking distances were determined by a standardised progressive treadmill test with a constant speed of $3.2 \mathrm{~km} / \mathrm{h}$ starting with $0 \%$ inclination, increasing every two minutes by 2\%.13, 15 For practical reasons, the maximal inclination was 10\% and the maximal duration of the test was 30 minutes (1600 meters)

The WIQ is a short and easy to fill out validated questionnaire for patients with peripheral arterial disease. ${ }^{16-18}$ The WIQ contains three domains to assess walking impairment: walking distance, walking speed and stair climbing. For each separate domain, a subscore of the Likert items was calculated. The mean of these domains represents the total WIQ score. ${ }^{19}$ We used a self administered revised version of the $\mathrm{WIQ},{ }^{18}$ recently adapted and validated for the European metric system and the Dutch language. ${ }^{20}$

The SF-36 is a general, frequently used quality of life questionnaire, validated for the Dutch language. ${ }^{21}$ The SF-36 contains 8 subscales that reflect mental and physical functioning (physical functioning, social functioning, physical role impairment,

emotional role impairment, mental health, vitality, pain and general health experience) and is calculated with a scoring algorithm. ${ }^{22}$

Statistical analysis

With a sample size of 81 patients per treatment arm, the trial had a power of $80 \%$, to demonstrate an increase in ACD of 150 meters (standard deviation (SD) 300 meters) with two-sided $a=0.025$. Based on a 15 to $20 \%$ withdrawal rate, 100 patients in each group had to be included.

Analysis was conducted according to the modified intention to treat principle. All data from patients who were randomized and completed treadmill assessment for quantifying walking distance after 12 months of treatment were included in the analysis.

Categorical variables were presented as frequencies with percentages, continuous variables were presented as means $( \pm S D)$ when normally distributed and as

medians with interquartile ranges (IQR) in case of a skewed distribution. For baseline characteristics comparisons between groups were performed using one way analysis of variance (ANOVA) for continuous variables and a Chi-square test for categorical variables.

Missing values of walking distances at 3, 6 and 9 months were imputed based on a multivariate linear regression analysis. A backward elimination method was used to identify variables included in the final regression equation to impute data. Baseline walking distance, age, chronic obstructive pulmonary disease (COPD) and cardiac disease as variables yielded $\mathrm{R}^{2} \mathrm{~s}$ for the ACD between 22.1\% and 28.1\%, which are comparable with an earlier report. ${ }^{23}$ Increases in walking distances within groups were analyzed with a repeated measurements ANOVA. Changes in walking distances between the groups over time were analyzed with a Wilcoxon rank sum test. Missing values of the WIQ and SF-36 were imputed with a mean substitution method. Differences of the WIQ and SF-36 within a group were analyzed with repeated measurements ANOVA. Statistical significance between the WA- and SET- with or without feedback group of the WIQ and the SF-36 was analyzed with repeated measurements analysis of covariance (ANCOVA) with the baseline measurement as covariate. Analyses were performed with SPSS 15.0 for Windows (SPSS Inc, Chicago, III). 


\begin{tabular}{llll}
\hline Table 1: baseline characteristics & & & \\
\hline & WA & & \\
& $\mathbf{n = 1 0 2}$ & $\begin{array}{l}\text { SET } \\
\mathbf{n = 1 0 9}\end{array}$ & $\begin{array}{l}\text { SET with feedback } \\
\mathbf{n = 9 3}\end{array}$ \\
\hline Men - \% & 55.9 & 72.5 & 60.2 \\
Age - mean (SD) & $66.9(8.6)$ & $66.1(9.0)$ & $65.6(10.5)$ \\
Body mass index - mean (SD)* & $28.2(4.7)$ & $27.4(4.2)$ & $28.2(5.1)$ \\
ABI - mean (SD) & $0.65(0.17)$ & $0.67(0.19)$ & $0.67(0.16)$ \\
ACD - median (IQR) & $240(160-345)$ & $260(167-395)$ & $250(160-340)$ \\
FCD - median (IQR) & $150(90-250)$ & $150(90-250)$ & $150(100-230)$ \\
Smoking - \% & & & \\
-current smoking & 47.1 & 38.5 & 41.9 \\
-former smoker & 41.2 & 49.5 & 46.2 \\
-never smoked & 9.8 & 12.0 & 4.3 \\
-unknown & 2.0 & 0 & 7.5 \\
Diabetes mellitus - \% & 23.5 & 25.7 & 18.3 \\
Orthopedic disease lower extremities - \% & 14.7 & 14.7 & 17.2 \\
Coronary heart disease - \% & 27.5 & 26.6 & 20.4 \\
Cerebrovascular accident or transient & 12.7 & 14.7 & 9.7 \\
ischemic attack - \% & & & \\
Chronic obstructive pulmonary disease - \% & 26.5 & 18.3 & 17.2 \\
\hline
\end{tabular}

WA denotes walking advice, SET supervised exercise therapy, SD standard deviation, ABI ankle brachial index, $A C D$ absolute claudication distance, IQR interquartile range, and FCD functional claudication distance

WA denotes walking advice, SET supervised exercise therapy, PAD peripheral arterial disease, and CVA cerebro vasculair accident.

* Body mass index is the weight in kilograms divided by the square of the body height in meters

\section{Results}

Study population

Between December 2005 to Mai 2008, 304 patients were enrolled in the study: 102 patients were included in the WA-group, 109 patients in the SET-group, and 93 patients in the SET with feedback group. The number of included patients per center is shown in the acknowledgments. Follow-up ended Mai 2009.

Baseline characteristics were generally well balanced among the study groups; there were more males in the SET-group and more current smokers in the WA-group. Median baseline walking distances were comparable in the three groups (Table 1).

Adherence to the intervention and follow-up

Five patients randomized to the WA-group started with SET during the course of the study; two on their own initiative and three after prescription of their vascular surgeon. Fifteen patients were lost to follow-up and 3 died, leaving 83 patients for analysis (Figure 1) Twenty-seven patients in the SET-group discontinued the program, 12 of them were lost to follow-up and 4 died. The remaining eleven patients discontinued the exercise program during the course of the study for other reasons than satisfaction with the regained walking distance but were eligible for analysis. Lack of motivation was the main recorded reason to discontinue the program. In total, 93 patients in the SET group were analysed.

In the SET with feedback group 27 patients stopped the SET program, 14 were lost to follow-up and 13 patients discontinued the program but were eligible for analysis. Of the analyzed patients $(n=76), 22(28.9 \%)$ patients reported not having used the PAM accelerometer at all, or only for part of the study year.

During the study, 9 (10.8\%) patients of the WA-group, 7 (7.5\%) of the SET-group and $6(7.9 \%)$ of the SET with feedback group underwent a peripheral vascular intervention due to worsening of complaints or dissatisfaction with the results of the exercise program $(\mathrm{P}=.68)$

Walking distances

The median ACD for the patients included in the modified intention to treat analysis increased from 260 to 400 meters, 280 to 620 meters, and 240 to 580 meters in the WA-, SET-, and SET with therapy feedback groups, respectively. The increase in median FCD showed a similar pattern; from 150 to 320 meters in the WA-group, from 160 to 440 meters in the SET-group, and from 140 to 475 meters in the SET with feedback group. Both, the ACD and the FCD increased significantly in all groups (Table 2). The difference between the groups in median changes of ACD and FCD between baseline and 12 months are presented in Figure 2. Patients following a SET program with or without feedback regained significantly more walking distance (ACD P $<.001$ and FCD 
$\mathrm{P}<.001)$ than patients in the WA-group. There was no difference in improvement of walking distances between the two SET-groups. The SET- and SET with feedback group increased approximately 200 meters (95\% confidence interval, 139 to 395) and 250 meter (95\% confidence interval, 124 to 376), respectively, more than the WA-group. Results did not substantially change after data imputation. Similar results between the WA- and SET-groups were obtained using ANCOVA with baseline walking distance as covariate.

Walking Impairment Questionnaire

In the WA-group the total WIQ score improved from 0.46 to 0.59 , in the SET-group from 0.48 to 0.69 , and in the SET with feedback group from 0.45 to 0.69. Repeated measurements ANCOVAs between the WA- and SET-group and the WA- and SET-group with feedback showed significantly more improvement in favor of the SET-group $(P=.008)$ and the SET with feedback group $(\mathrm{P}=.016)$ (Table 3). A Pearson correlation coefficient of $0.396(P<.001)$ was found between the change in ACD and the change in total WIQ score between 12 months and baseline.

\section{Quality of Life}

The physical summary score of the SF-36 improved significantly in the SET-groups (both $\mathrm{P}<.001$ ). This was mainly due to improvements in the subscales physical functioning and pain. While the physical health summary score showed a trend towards improvement in both SET-groups when compared with the WA-group, the subscale of physical functioning and pain showed significant improvements in both SETgroups. The mental health summary score did not improve over time. The 8 subscales and the physical and mental health components of the SF-36 are presented in Table 3.

Figure 2: Median changes in walking distances in meters with interquartile ranges between 12 months and baseline.

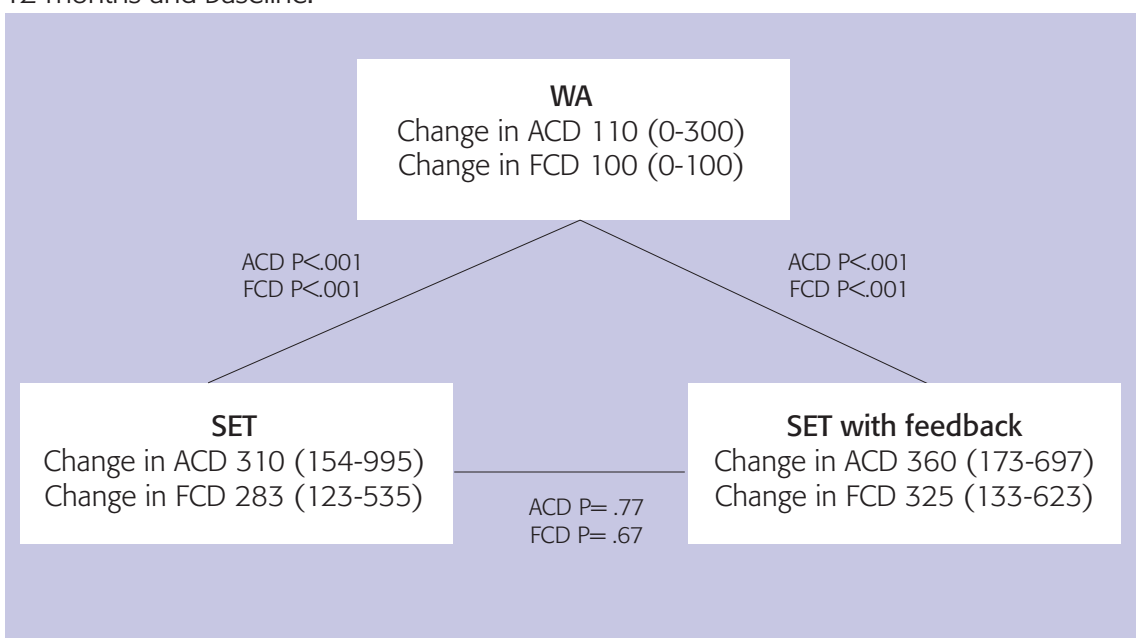

WA denotes walking advice, $A C D$ absolute claudication distance, FCD functional claudication distance and SET supervised exercise therapy

\begin{tabular}{|c|c|c|c|c|c|c|c|c|}
\hline & $\mathrm{n}$ & & Baseline & 3 months & $\begin{array}{l}6 \\
\text { months }\end{array}$ & $\begin{array}{l}9 \\
\text { months }\end{array}$ & 12 months & $\begin{array}{l}\mathrm{P}- \\
\text { value* }\end{array}$ \\
\hline \multirow[t]{2}{*}{ WA } & 83 & ACD & $\begin{array}{l}260 \\
(160-370)\end{array}$ & $\begin{array}{l}320 \\
(210-500)\end{array}$ & $\begin{array}{l}400 \\
(230-630)\end{array}$ & $\begin{array}{l}473 \\
(260-735)\end{array}$ & $\begin{array}{l}400 \\
(230-590)\end{array}$ & $<.001$ \\
\hline & & FCD & $\begin{array}{l}150 \\
(100-220)\end{array}$ & $\begin{array}{l}230 \\
(170-360\end{array}$ & $\begin{array}{l}320 \\
(180-480\end{array}$ & $\begin{array}{l}380 \\
(220-574\end{array}$ & $\begin{array}{l}320 \\
(180-500\end{array}$ & $<.001$ \\
\hline $\begin{array}{l}\text { Number of } \\
\text { patients completing } \\
1600 \text { meters during } \\
\text { treadmill testing }\end{array}$ & & & 0 & 2 & 9 & 5 & 8 & \\
\hline \multirow[t]{2}{*}{ SET } & 93 & ACD & $\begin{array}{l}280 \\
(170-405)\end{array}$ & $\begin{array}{l}530 \\
(385-890)\end{array}$ & $\begin{array}{l}635 \\
(440-1045)\end{array}$ & $\begin{array}{l}645 \\
(480-1235)\end{array}$ & $\begin{array}{l}620 \\
(420-1405)\end{array}$ & $<.001$ \\
\hline & & FCD & $\begin{array}{l}160 \\
(109-265)\end{array}$ & $\begin{array}{l}370 \\
(270-585)\end{array}$ & $\begin{array}{l}450 \\
(280-650)\end{array}$ & $\begin{array}{l}490 \\
(337-750)\end{array}$ & $\begin{array}{l}440 \\
(275-720)\end{array}$ & $<.001$ \\
\hline $\begin{array}{l}\text { Number of } \\
\text { patients completing } \\
1600 \text { meters during } \\
\text { treadmill testing }\end{array}$ & & & 0 & 8 & 14 & 20 & 23 & \\
\hline \multirow{2}{*}{$\begin{array}{l}\text { SET with } \\
\text { feedback }\end{array}$} & 76 & ACD & $\begin{array}{l}240 \\
(160-340)\end{array}$ & $\begin{array}{l}470 \\
(333-717)\end{array}$ & $\begin{array}{l}560 \\
(356-868)\end{array}$ & $\begin{array}{l}608 \\
(420-1076)\end{array}$ & $\begin{array}{l}580 \\
(445-1000)\end{array}$ & $<.001$ \\
\hline & & FCD & $\begin{array}{l}140 \\
(100-228)\end{array}$ & $\begin{array}{l}385 \\
(240-538)\end{array}$ & $\begin{array}{l}495 \\
(253-670)\end{array}$ & $\begin{array}{l}500 \\
(330-720)\end{array}$ & $\begin{array}{l}475 \\
(308-738)\end{array}$ & $<.001$ \\
\hline $\begin{array}{l}\text { Number of } \\
\text { patients completing } \\
1600 \text { meters during } \\
\text { treadmill testing }\end{array}$ & & & 0 & 9 & 13 & 13 & 14 & \\
\hline
\end{tabular}

WA denotes walking advice, SET supervised exercise therapy, ACD absolute claudication distance, and FCD functional claudication distance. * Repeated measurements ANOVA

\section{Discussion}

The results of this multicenter randomized clinical trial demonstrate that SET, provided by local physiotherapists, improves walking distances, WIQ scores, and scores on relevant domains of the SF-36 after 12 months. Compared with simple walking and exercise instructions combined with information leaflets, SET results in increase of the walking distance by approximately 200 meters, which reflects a clinically relevant improvement. The use of daily feedback by an accelerometer was not associated with further improvement.

The observed effect of SET versus WA on the ACD is well in line with results of a Cochrane meta-analysis, which calculated a difference of approximately 150 meters between SET and non supervised exercise programs in studies analysing small number of patients. 5 Similarly, six more recently published small trials (number of patients between 14 and 70) mainly a benefit of SET over non supervised exercise programs on walking distance. ${ }^{24-29}$ Studies assessing the effect of SET on quality of life expressed as relevant domains of the SF36 are scarce and inconclusive published data suggest that SET offers no to only modest 


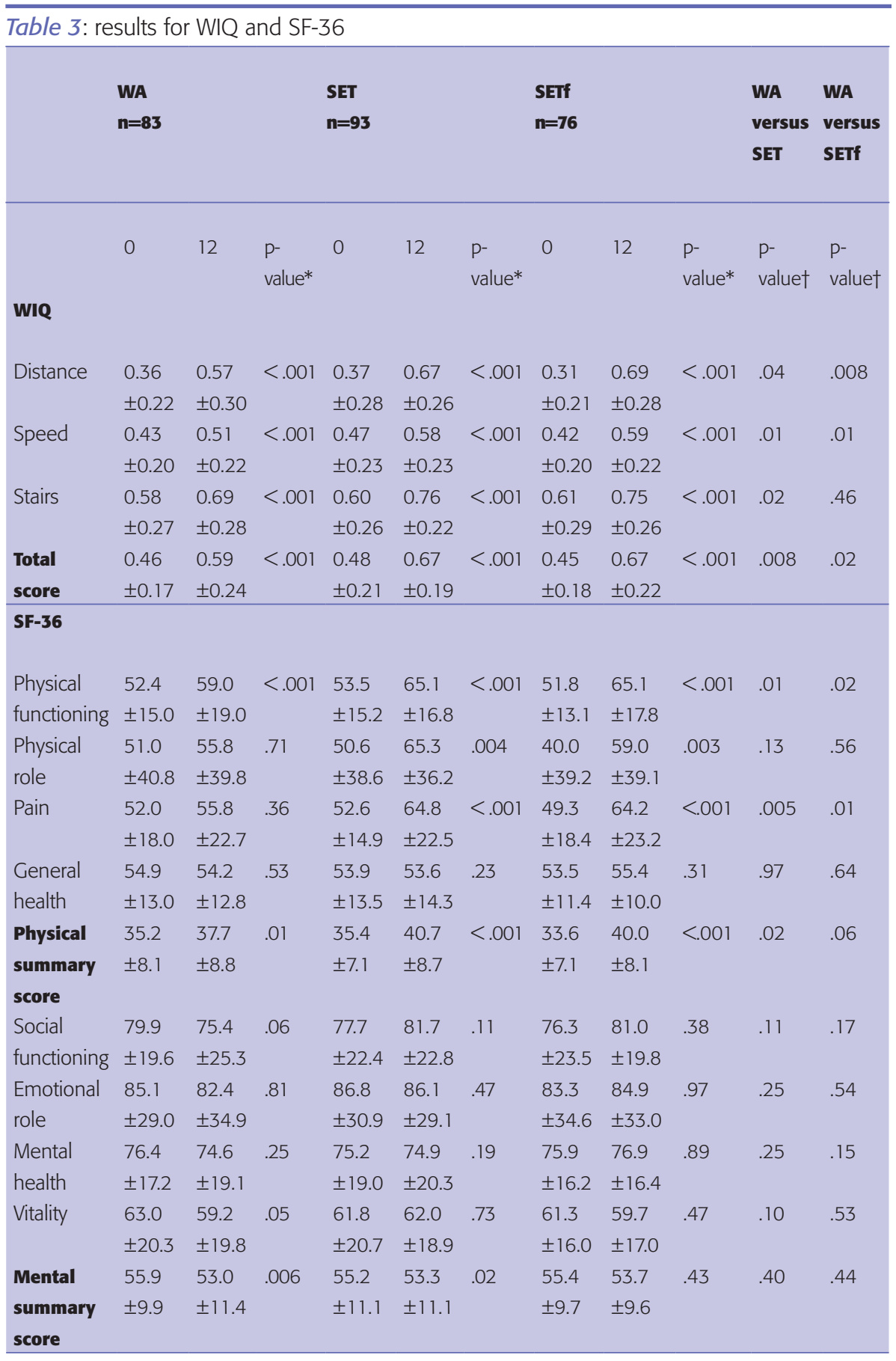

Data at 3, 6, and 9 months are not shown. WA denotes walking advice, SET supervised exercise therapy, SETf supervised exercise therapy with feedback, WIQ walking impairment questionnaire, and SF-36 short form 36

*Repeated measurements ANOVA

tRepeated measurements ANCOVA with baseline measurement as covariate reported mainly a benefit of SET over non supenised exercise of patients.5 Similarly, six more recently published small trials (number of patients between 14 and 70) reported improvement on quality of life compared with non-supervised exercise programs., 28,30 While patients who used an accelerometer for feedback might have been more active, ${ }_{1}^{31}$ a recent meta-analysis suggests that training more than 3 times per week does not provide further benefit for patients with intermittent claudication.32

In this large randomized clinical we were able to demonstrate a beneficial effect of SET compared with WA on walking distance. The benefits were apparent as early as 3 months after initiation of therapy, and persisted for at least 12 months. More importantly, SET offered a sustained benefit on physical domains of quality of life, which was not seen after nonsupervised training. Participating in a trial that stimulates physical activity affects patients behaviour, ${ }^{33}$ and patients who were willing to participate in this study might have had an intrinsic motivation to increase their activity level, which may limit generalizability. Finally, due to the nature of the interventions this was an open study. While we attempted to obtain an unbiased observation of the $A C D$, it is difficult to ascertain complete blinding of this outcome measurement.

The results of this large randomized clinical trial are consistent with a meta-analysis of previously performed smaller studies. ${ }^{5}$ Both indicate that SET is an effective treatment for intermittent claudication and should be offered as first line treatment. However, the availability of SET in clinical practice is far from optimal. ${ }^{44,35}$ This can be related to reimbursement policies of insurance companies and availability of adequate trained professionals who can provide SET. Hence, there seems to be an obligation for professionals in the vascular field to take action to make this effective intervention available for all patients with intermittent claudication.

In conclusion, supervised exercise therapy is more effective than walking advice alone in improving walking distance, WIQ scores, and quality of life as assessed in relevant domains of the SF-36 for patients with intermittent claudication. A supervised exercise program should be made available for all patients with intermittent claudication.

\section{Acknowledgments}

We thank all patients who participated in the EXITPAD study and the investigators who assisted at collaborating sites.

\section{Participating centres}

Participating investigators and number of patients included in the EXITPAD study. The Netherlands: J.A.W. Teijink and A. Habets, Atrium medical centre Parkstad, Heerlen (125), R.J. van Det, A. Stam, and M. Blokhuis, Medical Spectrum Twente, Enschede (46), P.J.G. Jörning, I. Siebelt, L. Kasperink, and R. Haze, Isala Klinieken, Zwolle (30), A.C. Vahl and J.M. de Vries, Onze Lieve Vrouwe Gasthuis, Amsterdam (27), R.P. Tutein Nolthenius, I Nicaise, and P de Jong, Albert Schweitzer hospital, Dordrecht (23), P.W.M. Cuypers and G. Kiebert, Catharina Hospital, Eindhoven (22), L.H.J.M. van den Akker and W. Hermans, Orbis Medical Centre, Sittard (14), M.G. Samyn, G de Bruijn S. Peredda, and S. Wijnands, VieCurie, Venlo (7), P. Brummel, S. Brandwijk, and P. Kosters, Franciscus Hospital, Roosendaal (6), Th.A.A. van den Broek, G.A. Vos, M. van Boxtel, and R. Smit, Waterland Hospital, Purmerend (3), and G.H. Ho, L. Schoones, and M. Oerlemans, Amphia Hospital, Breda (1) 


\section{References}

1. Norgren L, Hiatt WR, Dormandy JA, Nehler MR, Harris KA, Fowkes FG. Inter-Society Consensus for the Management of Peripheral Arterial Disease (TASC II). J Vasc Surg. 2007;45 Suppl S:S5-67.

2. Vahl AC, Reekers JA. The guideline 'Diagnosis and treatment of peripheral artery disease of the lower extremities' of The Netherlands Surgical Society]. Ned Tijdschr Geneeskd. 2005;149:1670-1674.

3.Leng GC, Fowler B, Enst E. Exercise for intermittent claudication. Cochrane database of systematic reviews (Online). 2000(2):CD000990.

4. Bartelink ML, Stoffers HE, Biesheuvel CJ, Hoes AW. Walking exercise in patients with intermittent claudication. Experience in routine clinical practice. Br J Gen Pract. 2004;54:196-200.

5. Bendermacher BL, Willigendael EM, Teijink JA, Prins MH. Supenised exercise therapy versus non-supenised exercise therapy for intermittent claudication. Cochrane database of systematic reviews (Online). 2006(2):CD005263.

6. Willigendael EM, Bendermacher BL, van der Berg C, et al. The development and implementation of a regiona network of physiotherapists for exercise therapy in patients with peripheral arterial disease, a preliminary report. BMC Health Serv Res. 2005;5:49.

7. Bendermacher BL, Willigendael EM, Nicolai SP, et al. Supervised exercise therapy for intermittent claudication in a community-based setting is as effective as clinicbased. J Vasc Surg. 2007;45:1192-1196.

8. Kruidenier LM, Nicolai SP, Hendriks E, Bollen EC, Prins MH, Teijink JA. Supervised exercise therapy for intermittent claudication in daily practice. J Vasc Surg. 2009;49:363-370.

9. de Booys $\mathrm{M}$, van Zenderen $\mathrm{B}$, Zwiebel F. [exercise training: walking, walking, walking] 19th ed. Bitthoven, The Netherlands: Vereniging van vaatpatënten, 2006:1-23.

10. Jongert MWA, Hendriks HJM, Van Hoek J, et al. [Dutch guideline Intermittent Claudication]. Ned Tijdschr Fysiother 2003;Suppl:3-58.
11. Homepage Personal Activity Monitor (PAM). Doorwerth, The Netherlands, 2009. (Accessed August 19, 2009, at http://mwn.pam.com/.)

12. Slootmaker SM, Chin A Paw MJ, Schuit AJ, van Mechelen W, Koppes LL. Concurrent validity of the PAM accelerometer relative to the MTI Actigraph using oxygen consumption as a reference. Scan J Med Sci Sports. 2007;19:36-43.

13. Nicolai SP, Viechtbauer W, Kruidenier LM, Candel MJ, Prins MH, Teijink JA. Reliability of treadmill testing in peripheral arterial disease: a meta-regression analysis. IVasc Surg. 2009:50:322-329.

14. Kruidenier LM, Nicolai SP, Willigendael EM, de Bie RA Prins $\mathrm{MH}$, Teijink JA. Functional claudication distance: a reliable and valid measurement to assess functiona limitation in patients with intermittent claudication. BMC cardiovascular disorders. 2009;9:9.

15. Gardner AW, Skinner JS, Cantwell BW, Smith LK. Progressive vs single-stage treadmill tests for evaluation of claudication. Med Sci Sports Exerc. 1991;23:402-408.

16. Regensteiner JG, Steiner JF, Panzer R, Hiatt WR. Evaluation of walking impairment by questionnaire in patients with peripheral arterial disease. J Vasc Med Biol. 1990:2:142-152.

17. McDermott MM, Liu K, Guralnik JM, Martin GJ, Criqui $\mathrm{MH}$, Greenland P. Measurement of walking endurance and walking velocity with questionnaire: validation of the walking impairment questionnaire in men and women with peripheral arterial disease. J Vasc Surg. 1998;28(6):1072-1081.

18. Coyne KS, Margolis MK, Gilchrist KA, et al. Evaluating effects of method of administration on Walking Impairment Questionnaire. J Vasc Surg. 2003:38(2):296-304.

19. Nicolai SP, Kruidenier LM, Rouwet EV, Graffius K, Prins $\mathrm{MH}$, Teiijink JA. The walking impairment questionnaire an effective tool to assess the effect of treatment in patients with intermittent claudication. J Vasc Surg. 2009;50:89-94.
20. Verspaget $M$, Nicolai $S P$, Kruidenier $L M$, Welten $R$ Prins MH, Teijink JA. Validation of the Dutch version of the Walking Impaiment Questionnaire Eur JVasc Endovasc Surg. 2009:37:56-61.

21. Aaronson NK, Muller M, Cohen PD, et al. Translation, validation, and norming of the Dutch language versio of the SF-36 Health Survey in community and chronic disease populations. J Clin Epidemiol. 1998;51:10551068.

22. Ware JE, Jr, Kosinski M, Bayliss MS, McHorney CA Rogers WH, Raczek A. Comparison of methods for the scoring and statistical analysis of SF-36 health profile and summary measures: summary of results from the Medical Outcomes Study. Med Care. 1995;33(4 Suppl):AS264-279.

23. Kruidenier LM, Nicolaii SP, Ten Bosch JA, de Bie RA, Prins MH, Teijink JA. Predictors of Walking Distance After Supenised Exercise Therapy in Patients with Intermittent Claudication. Eur J Vasc Endovasc Surg. 2009:38:449-5

24. Crowther RG, Spinks WL, Leicht AS, Sangla K, Quigley $F$, Golledge J. Effects of a long-term exercise program on lower limb mobility physiological responses, walking performance, and physical activity levels in patients with peripheral arterial disease. J Vasc Surg. 2008;47:303-309.

25. Hobbs SD, Marshall T, Fegan C, Adam DJ, Bradbury AW. The constitutive procoagulant and hypofibrinolytic state in patients with intermittent claudication due to infrainguinal disease significantly improves with percutaneous transluminal balloon angioplasty. J Vasc Surg. 2006;43:40-46.

26. Hobbs SD, Marshall T, Fegan C, Adam DJ, Bradbury AW. The effect of supenised exercise and cilostazol on coagulation and fibrinolysis in intermittent claudication: a randomized controlled trial. J Vasc Surs 2007:45:65-70.

27. Hodges LD, Sandercock GR, Das SK, Brodie DA Randomized controlled trial of supervised exercise to evaluate changes in cardiac function in patients with peripheral atherosclerotic disease. Clin Physiol Funct maging. 2008;28:32-37.
28. Lee HL, Mehta T, Ray B, Heng MS, McCollum PT, Chetter IC. A non-randomised controlled trial of the clinical and cost effectiveness of a Supervised Exercise Programme for claudication. Eur J Vasc Endovasc Surg 2007;33:202-207

29. Stewart AH, Smith FC, Baird RN, Lamont PM. Local versus systemic mechanisms underlying supenised exercise training for intermittent claudication. Vasc Endovascular Surg. 2008;42:314-320.

30. Imfeld S, Singer L, Degischer S, et al. Quality of life improvement after hospital- based rehabilitation or home-based physical training in intermittent claudication. VASA. 2006;35:178-184.

31. Bravata DM, Smith-Spangler C, Sundaram V, et al. Using pedometers to increase physical activity and improve health: a systematic review. JAMA 2007;298(19):2296-2304

32. Bulmer AC, Coombes IS. Optimising exercise training in peripheral arterial disease. Sports Med 2004;34:983-1003

33. van Sluijs EM, van Poppel MN, Twisk JW, van Mechelen W. Physical activity measurements affected participants' behavior in a randomized controlled trial. J Clin Epidemiol 2006;59:404-411.

34. Willigendael EM, Bendermacher BL, Nicolai SP, Teijink JA. [Supenised exercise therapy for patients with intermittent claudication; the necessity to train physiotherapists after release of the national guideline] Ned Tijdschr Fysiother. 2007:117:10-14

35. Shalhoub J, Hamish M, Davies AH. Supervised exercise for intermittent claudication - an under-utilised tool. Ann R Coll Surg Engl 2009;91:473-6

36. Moher D, Schulz KF, Altman DG. The CONSORT statement: revised recommendations for improving the quality of reports of parallel-group randomised trials. Lancet. 2001;357:1191-1194.

37. Altman DG, Schulz KF, Moher D, et al. The revised CONSORT statement for reporting randomized trials: explanation and elaboration. Ann Internal Med 2001;134:663-694 
CHAPTER 10
Optimizing supervised
exercise therapy
for patients
with intermittent
claudication 


\section{Abstract}

Background: The first-line intervention for intermittent claudication is usually supervised exercise therapy (SET). The literature describes a range of exercise programs varying in setting, duration and content. The purpose of the present study was to examine the exercise protocols offered, and to identify the impact of the intensity of the SET programs (in terms of frequency, duration, and type of exercise) on improvements in walking distance (response) in the first 3 months. The present study is part of the Exercise Therapy in Peripheral Arterial Disease (EXITPAD) study, a multicenter randomized clinical trial comparing the effects of SET provided by regional physiotherapists, with or without daily feedback, on the level of activities with the effects of walking advice.

Methods: The analysis included patients randomized to receive SET with or without feedback. The physiotherapists administering the SET were asked to fill out therapy evaluation sheets stating frequency, duration, and type of exercises. The relationship between training volume and the impact on walking distance was explored by dividing training volume data into tertiles and relating them to the median change in maximal walking distance at 3 and 12 months.

Results: Data of 169 patients were included in the analysis. A SET program consisting of at least 2 training sessions per week each lasting over 30 minutes, during the first 3 months of a one-year program tailored to individual patients' needs led to better results in terms of walking distance after 3 and 12 months than the other variants. The results of our analysis dividing training volume into tertiles suggest that there is a relationship between training volume and improvement in walking distance and that at least 590 minutes of training should be offered in the first 3 months. No differences were found between program involving only walking and a combination of exercises, nor between individual and group training.

Conclusion: A SET program consisting of at least 2 training sessions a week, each lasting over 30 minutes, should be offered during the first 3 months of the SET program to optimize improvement in terms of maximum walking distance.

\section{Introduction}

Symptomatic peripheral arterial disease (PAD) is characterized by leg pain during exertion, which disappears in rest. Atherosclerosis leads to narrowing of the arteries, resulting in ischemic pain. Treatment for symptomatic relief consists of conservative and / or invasive therapy. The first-line conservative therapy usually takes the form of supervised exercise therapy (SET), which is known to increase walking distance and quality of life.

SET has been shown to offer added benefit over unsupervised exercise therapy. ${ }^{2}$ The literature describes a range of exercise programs, varying in terms of setting, duration, and content. In the Netherlands, SET is prescribed according to the evidencebased guideline on 'Intermittent Claudication' of the Royal Dutch Society for Physical Therapy. ${ }^{3}$ This guideline provides recommendations about the content and duration of SET. The main goals are symptom relief and cardiovascular risk factor management. Objectives include increasing maximum walking distance, increasing aerobic endurance, increasing pain tolerance, reducing fear of pain, improving gait pattern and walking efficiency, improving patient-specific activity limitations (stair climbing etc), providing information, and inducing lifestyle changes. However, the guideline prescribes no stringent exercise protocol, and physiotherapists are advised to tailor the content and duration of the program to the individual patient's needs. The aim of the present study was to survey the tailored exercise protocols that are being offered and to identify the impact of the intensity of these SET programs, in terms of frequency, duration and type of exercise, on the improvement in walking distance.

\section{Methods}

This study involved a secondary analysis of data from the Exercise Therapy in Peripheral Arterial Disease (EXITPAD) study, a multicenter randomized clinical trial comparing the effects of SET provided by regional physiotherapists, with or without daily therapy feedback, with the effects of walking advice.'

A more extensive description of the methods of the EXITPAD study and its results in terms of walking distances and quality of life are described in Chapter 9. There were no significant differences between the SET groups with and without feedback in terms of walking distances of the SET- and SET with feedback groups.'

Patients

Patients with $P A D$, defined as an ankle brachial index of $<0.9$, who were considered for conservative treatment were included at the vascular outpatient dinics of 11

Dutch hospitals. Inclusion criteria were: patients having PAD according to according to Fontaine stage II and an absolute claudication distance (ACD) of $500 \mathrm{~m}$ as assessed by a graded standardized treadmill test. ${ }^{4}$ Exclusion criteria included prior exercise therapy, previous peripheral vascular interventions, insufficient command of the Dutch language, serious cardiopulmonary limitations (NYHA-3-4), previous amputation, psychiatric instability, and other serious co-morbidities prohibiting physical training. After the vascular surgeon had confirmed eligibility, patients were centrally randomized. An independent researcher used a computer to generate a randomization list with a block size of 9 , stratified for each study center. The sequence was concealed until interventions had been assigned. 
Intervention

Patients randomized to SET (with or without therapy feedback) were referred to local physiotherapists participating in the EXITPAD study. All participating physiotherapists were trained according to the guideline on 'Intermittent claudication' of the Royal Dutch Society for Physical Therapy ${ }^{3}$ and therapists were asked to administer SET according to this guideline. A summary of the guideline is presented in Table 1. The main objectives of the guideline are to increase maximum walking distance, aerobic endurance and pain tolerance, to reduce fear of pain, to improve gait pattern and walking efficiency, to improve patient-specific activity limitations (stair climbing, etc), to provide information, and to induce lifestyle changes. The guideline recommends increasing the maximum walking distance by interval treadmill walking up to nearmaximum pain in 5 minutes, with at least 3 to 5 repetitions. Activities such as walking, cycling / spinning, rowing, stepping, and sports activities in general are recommended to increase aerobic endurance.

\section{Table 1: Summary of the Royal Dutch Society for Physical Therapy's guideline on} 'Intermittent Claudication'

\begin{tabular}{|c|c|c|}
\hline $\begin{array}{l}\text { Facilities for } \\
\text { SET }\end{array}$ & Equipment & $\begin{array}{l}\text { Treadmill; cycle ergometer; indoor walking space; } \\
\text { small-group } \\
\text { exercise room }\end{array}$ \\
\hline \multirow{5}{*}{$\begin{array}{l}\text { Diagnostic } \\
\text { process }\end{array}$} & Education & $\begin{array}{l}\text { Specific education to provide exercise therapy according to the } \\
\text { recommendations of the guideline is required }\end{array}$ \\
\hline & $\begin{array}{l}\text { Referral } \\
\text { (general } \\
\text { practitioner / } \\
\text { specialist) }\end{array}$ & $\begin{array}{l}\text { Fontaine classification; ankle-brachial index }(<0.9) \text {; walking } \\
\text { impairment; cardiac risk and capacity; contra-indications }\end{array}$ \\
\hline & $\begin{array}{l}\text { Medical } \\
\text { history }\end{array}$ & $\begin{array}{l}\text { Disease-specific; co-morbidities; patient-specific complaints } \\
\text { scale }\end{array}$ \\
\hline & $\begin{array}{l}\text { Physical } \\
\text { examination }\end{array}$ & Disease-specific; co-morbidities \\
\hline & $\begin{array}{l}\text { Functional } \\
\text { examination }\end{array}$ & Maximum treadmill test; gait and specific function analysis \\
\hline \multirow[t]{4}{*}{$\begin{array}{l}\text { Therapeutic } \\
\text { process }\end{array}$} & Objectives & $\begin{array}{l}\text { Increase maximum walking distance; increase aerobic } \\
\text { endurance; increase pain tolerance and reduce fear of pain; } \\
\text { improve gait } \\
\text { pattern and specific activities (stair climbing, etc); provide } \\
\text { information and induce lifestyle changes }\end{array}$ \\
\hline & $\begin{array}{l}\text { Behavioral } \\
\text { change }\end{array}$ & $\begin{array}{l}\text { Increase pain tolerance; reduce fear of pain; induce active } \\
\text { lifestyle and improve risk factors }\end{array}$ \\
\hline & $\begin{array}{l}\text { Supply } \\
\text { information }\end{array}$ & Disease-specific; exercise therapy; lifestyle \\
\hline & $\begin{array}{l}\text { Therapy } \\
\text { mode and } \\
\text { intensity }\end{array}$ & $\begin{array}{l}\text { Primarily walking up to maximum or submaximum pain; at } \\
\text { least } 6 \text { months; at least } 30 \text { minutes per session; walking } 3 \\
\text { times a week (supervised or homework) }\end{array}$ \\
\hline
\end{tabular}

ACSM denotes American College of Sports Medicine, SET supervised exercise therapy
Patients were evaluated at baseline and at 3, 6, 9 and 12 months of follow-up, by an observer blinded to group assignment. Patients performed a standardized graded treadmill test at a speed of $3.2 \mathrm{~km} / \mathrm{h}$, starting at 0\% incline and increasing by $2 \%$ every 2 minutes. ${ }^{4}$ The incline and test duration were limited, for practical reasons, to $10 \%$ and 30 minutes (1600 m), respectively. The primary outcome measure was the change in $A C D$, defined as the maximal walking distance limited by claudication pain. The secondary outcome measure was the change in functional claudication distance (FCD), defined as the distance at which the patient prefers to stop because of claudication pain.

Detailed therapy evaluation and documentation sheets were filled out by the physiotherapists after $6,12,26$, and 52 weeks of therapy, including questions about frequency, duration and content of the therapy.

Statistical analysis

Our analysis of the EXITPAD study data only included patients randomized to exercise therapy groups in a physiotherapeutic setting with and without therapy feedback. Analysis was conducted according to the modified intention to treat principle. The analysis included al data from patients who had been randomized and had completed treadmill assessment to quantify walking distance after 12 months of treatment

Training volume (expressed in minutes) was calculated using the following equation: frequency (days per week) * session frequency (sessions per day) * session duration (minutes per session) $*$ total duration (weeks). ${ }^{5}$ Data were extracted from the therapy evaluation and documentation sheets provided by the physiotherapists.

Categorical variables are presented as means $( \pm S D)$ when normally distributed and as medians with interquartile ranges (IQR) in the case of a skewed distribution. Groups were compared using oneway analysis of variance (ANOVA).

The guideline for physiotherapists recommends starting with SET, and after a few weeks tailoring the program to the individual needs of the patient, meaning that patients who perform well and comply with the exercise recommendations can phase down the therapy frequency sooner than other patients. We explored whether there was a relationship between the training volume offered and the effect in terms of walking distance by analyzing training volume data for the first 3 months, that is, the period in which the training program had hardly been adapted to the individual needs of the patients yet. For the purpose of this analysis, the training volume offered was divided into tertiles and related to the median change in $A C D$ after 3 and 12 months. Differences in walking distance between groups were analyzed with a Wilcoxon rank sum test.

\section{Results}

Study population

One hundred and nine patients were randomized to the SET without feedback group, and 93 to the SET with feedback group. Table 2 presents baseline characteristics of all included patients. Data from 169 patients, comprising 93 patients from the SET without feedback group and 76 from the SET with feedback group were included in the modified intention to treat analysis. Median walking distances increased significantly in both the SET without feedback group and the SET with feedback group, from 280 to $620 \mathrm{~m}$ and from 240 to $580 \mathrm{~m}$ respectively. However, there was no difference in improvement between the SET without feedback and SET with feedback groups.' 
Content of the exercise program

Physiotherapists were asked to fill out therapy evaluation sheets. After 6, 12, 26, and 52 weeks, we had collected 149, 137, 134, and 113 evaluation sheets, respectively. The content of the SET program, expressed as the number of training sessions, duration of training sessions and training volume, did not differ between the two groups (Table 3).

Relation between exercise intensity and results

In view of the similarities in terms of improved walking distance' and the exercise program offered in the two treatment groups, data for both groups were analyzed together.

\begin{tabular}{lll}
\hline Table 2: Baseline characteristics & & \\
& & \\
& SET & \\
& $n=109$ & $\begin{array}{l}\text { SET with } \\
\text { feedback } \\
n=93\end{array}$ \\
& & \\
& & \\
\hline Men - \% & 72.5 & 60.2 \\
Age - mean (SD) & $66.1(9.0)$ & $65.6(10.5)$ \\
Body mass index - mean (SD)* & $27.4(4.2)$ & $28.2(5.1)$ \\
ABI - mean (SD) & $0.67(0.19)$ & $0.67(0.16)$ \\
ACD - median (IQR) & $260(167-395)$ & $250(160-340)$ \\
FCD - median (IQR) & $150(90-250)$ & $150(100-230)$ \\
Smoking - \% & & \\
-current smoking & 38.5 & 41.9 \\
-former smoker & 49.5 & 46.2 \\
-never smoked & 12.0 & 4.3 \\
-unknown & 0 & 7.5 \\
Diabetes mellitus - \% & 25.7 & 18.3 \\
Orthopedic disease of lower extremities - \% & 14.7 & 17.2 \\
Coronary heart disease - \% & 26.6 & 20.4 \\
Cerebrovascular accident or transient ischemic attack - \% & 14.7 & 9.7 \\
Chronic obstructive pulmonary disease - \% & 18.3 & 17.2 \\
\hline
\end{tabular}

SET denotes supervised exercise therapy, SD standard deviation, $A B I$ ankle brachial index, $A C D$ absolute claudication distance, IQR interquartile range, and FCD functional claudication distance * Body mass index is the weight in kilograms divided by the square of the body height in meters

The mean number of training sessions during the first 3 months was related to the median increase in ACD. Having a SET session once a week resulted in a median ACD increase of $205 \mathrm{~m}$ after 3 months, while 2 and 3 training sessions a week increased the ACD by a median of 273 and $245 \mathrm{~m}$, respectively. Two or more SET training sessions a week during the first 3 months yielded significantly better results in terms of walking distance after 12 months than one training session a week in this period (Table 4).

\begin{tabular}{|c|c|c|c|c|c|c|c|c|c|}
\hline & \multicolumn{4}{|l|}{ SET } & \multicolumn{5}{|c|}{ SET with feedback } \\
\hline & Week & Week & Week & Week & Week & Week & Week & Week & P-value* \\
\hline & 6 & 12 & 26 & 52 & 6 & 12 & 26 & 52 & SET and \\
\hline & $n=79$ & $n=74$ & $\mathrm{~N}=73$ & $\mathrm{~N}=59$ & $n=70$ & $n=63$ & $\mathrm{~N}=61$ & $\mathrm{~N}=54$ & SETf \\
\hline $\begin{array}{l}\text { Sessions per } \\
\text { week - mean } \\
\text { (SD) }\end{array}$ & $\begin{array}{l}1.82 \\
(0.60)\end{array}$ & $\begin{array}{l}1.61 \\
(0.72)\end{array}$ & $\begin{array}{l}1.15 \\
(0.75)\end{array}$ & $\begin{array}{l}0.82 \\
(0.74)\end{array}$ & $\begin{array}{l}1.64 \\
(0.58)\end{array}$ & $\begin{array}{l}1.41 \\
(0.69)\end{array}$ & $\begin{array}{l}1.00 \\
(0.69)\end{array}$ & $\begin{array}{l}0.75 \\
(0.66)\end{array}$ & 0.34 \\
\hline $\begin{array}{l}\text { Cumulative } \\
\text { sessions per } \\
\text { week - mean } \\
\text { (SD) }\end{array}$ & $\begin{array}{l}10.9 \\
(3.6)\end{array}$ & $\begin{array}{l}20.6 \\
(7.2)\end{array}$ & $\begin{array}{l}37.0 \\
(16.6)\end{array}$ & $\begin{array}{l}59.0 \\
(32.2)\end{array}$ & $\begin{array}{l}9.8 \\
(3.5)\end{array}$ & $\begin{array}{l}18.4 \\
(7.0)\end{array}$ & $\begin{array}{l}33.5 \\
(14.8)\end{array}$ & $\begin{array}{l}55.6 \\
(29.5)\end{array}$ & 0.58 \\
\hline $\begin{array}{l}\text { Duration per } \\
\text { session - } \\
\text { mean (SD) }\end{array}$ & $\begin{array}{l}38.6 \\
(14.8)\end{array}$ & $\begin{array}{l}41.3 \\
(13.5)\end{array}$ & $\begin{array}{l}39.6 \\
(18.8)\end{array}$ & $\begin{array}{l}36.3 \\
(21.5)\end{array}$ & $\begin{array}{l}39.7 \\
(15.0)\end{array}$ & $\begin{array}{l}39.6 \\
(17.3)\end{array}$ & $\begin{array}{l}37.7 \\
(18.2)\end{array}$ & $\begin{array}{l}36.7 \\
(19.5)\end{array}$ & \\
\hline $\begin{array}{l}\text { Cumulative } \\
\text { training } \\
\text { volume }^{1} \text { - } \\
\text { mean (SD) }\end{array}$ & $\begin{array}{l}431 \\
(224)\end{array}$ & $\begin{array}{l}867 \\
(415)\end{array}$ & $\begin{array}{l}1557 \\
(924)\end{array}$ & $\begin{array}{l}2740 \\
(1840)\end{array}$ & $\begin{array}{l}403 \\
(189)\end{array}$ & $\begin{array}{l}799 \\
(404)\end{array}$ & $\begin{array}{l}1481 \\
(868)\end{array}$ & $\begin{array}{l}2479 \\
(1628)\end{array}$ & 0.43 \\
\hline
\end{tabular}

SET denotes supervised exercise therapy and SETf supervised exercise therapy with feedback * repeated measurements ANOVA

${ }^{\top}$ Volume $=$ cumulative number of trainings $*$ duration

The duration of the training sessions during the first 3 months was divided into three categories: 30 minutes or less, between 30 and 45 minutes, and over 45 minutes. Training sessions lasting less than 30 minutes led to an improvement in the median ACD of 230 and $240 \mathrm{~m}$ at 3 and 12 months, respectively. Training sessions with a longer duration (> 30 minutes) yielded significantly better increases in median ACD: from 270 to $485 \mathrm{~m}$ for sessions lasting between 30 and 45 minutes and from 290 to $390 \mathrm{~m}$ for sessions lasting over 45 minutes, compared with training sessions lasting 30 minutes or less (230 to $240 \mathrm{~m}$ ) $(p=.021$ after 3 months and $p=.017$ after 12 months).

To explore the relationship between the training volume offered, which depends on frequency and duration, and the effect of therapy in terms of walking distance, we divided training volume into tertiles. In the first tertile, the training volume offered in the first 3 months ranged from 0 to 590 minutes, in the second tertile from 591 to 1000 minutes, and in the third tertile from 1001 to 2160 minutes. Table 4 relates the tertiles of training volume to the median change in ACD after 3 and 12 months. The median change in walking distance in the first tertile was $158 \mathrm{~m}$ after 3 months and $218 \mathrm{~m}$ after 12 months. Across the three tertiles, the median change in walking distance significantly improved when comparing the tertile with the lowest training volume with the two tertiles with the higher training volumes (with median changes of 300 and $470 \mathrm{~m}$, and 265 and $445 \mathrm{~m}$ in the second and third tertiles after 3 and 12 months, respectively) ( $p=.002$ at 3 months and $p=.003$ at 12 months). This indicates that offering a training volume higher than 590 minutes in the first 3 months was associated with greater improvement in walking distance, in the short term as well as after 12 months. 
Table 4: Cumulative training volume and mean number of training sessions during the first 3 months, combining the SET without feedback and SET with feedback groups.

Mean number of training sessions per week during the first 3 months

\begin{tabular}{|c|c|c|c|c|}
\hline & 1 & 2 & 3 or more & $\begin{array}{l}\text { p-value* } 1 \text { session } \\
\text { versus } \\
2 \text { or more sessions }\end{array}$ \\
\hline & $n=44$ & $n=81$ & $\mathrm{n}=10$ & \\
\hline $\begin{array}{l}\text { Median change in ACD } \\
\text { between } 3 \text { months } \\
\text { and baseline } \\
\text { Median change in ACD }\end{array}$ & $\begin{array}{l}205 \\
\text { (80 to 398) }\end{array}$ & $\begin{array}{l}273 \\
(165 \text { to } 545)\end{array}$ & $\begin{array}{l}245 \\
(155 \text { to } 524)\end{array}$ & .052 \\
\hline $\begin{array}{l}\text { between } 12 \text { months } \\
\text { and baseline }\end{array}$ & $\begin{array}{l}245 \\
\text { (115 to } 445)\end{array}$ & $\begin{array}{l}480 \\
\text { (195 to } 1100)\end{array}$ & $\begin{array}{l}425 \\
\text { (120 to } 719)\end{array}$ & .004 \\
\hline \multicolumn{5}{|c|}{ Duration of training sessions per week during the first 3 months } \\
\hline & $\leq 30$ minutes & $\begin{array}{l}\text { Between } 30 \\
\text { and } 45 \text { minutes }\end{array}$ & $>45$ minutes & $\begin{array}{l}\text { p-value* } \leq 30 \\
\text { minutes versus }> \\
30 \text { minutes }\end{array}$ \\
\hline Median chanoe in ACD & $\mathrm{n}=51$ & $n=36$ & $n=45$ & \\
\hline $\begin{array}{l}\text { between } 3 \text { months } \\
\text { and baseline } \\
\text { Median change in ACD }\end{array}$ & $\begin{array}{l}230 \\
(100 \text { to } 350)\end{array}$ & $\begin{array}{l}270 \\
\text { (155 to } 733)\end{array}$ & $\begin{array}{l}290 \\
(150 \text { to } 510)\end{array}$ & .021 \\
\hline $\begin{array}{l}\text { between } 12 \text { months } \\
\text { and baseline }\end{array}$ & $\begin{array}{l}240 \\
\text { (130 to } 510 \text { ) }\end{array}$ & $\begin{array}{l}485 \\
\text { (267 to } 1138 \text { ) }\end{array}$ & $\begin{array}{l}390 \\
(170 \text { to } 1165)\end{array}$ & .017 \\
\hline \multicolumn{5}{|c|}{ Cumulative training volume of therapy in the first 3 months } \\
\hline & $\begin{array}{l}\text { Tertile } 1 \\
0-590\end{array}$ & $\begin{array}{l}\text { Tertile } 2 \\
\text { 591-1000 }\end{array}$ & $\begin{array}{l}\text { Tertile } 3 \\
1001-2160\end{array}$ & $\begin{array}{l}\text { p-value* tertile } 1 \\
\text { versus tertiles } 2 \\
\text { and } 3\end{array}$ \\
\hline & $\mathrm{n}=40$ & $n=45$ & $\mathrm{n}=44$ & \\
\hline $\begin{array}{l}\text { Median change in ACD } \\
\text { between } 3 \text { months } \\
\text { and baseline }\end{array}$ & $\begin{array}{l}158 \\
\text { (81 to 300) }\end{array}$ & $\begin{array}{l}300 \\
\text { (177 to } 780)\end{array}$ & $\begin{array}{l}265 \\
\text { (173 to } 475 \text { ) }\end{array}$ & .002 \\
\hline $\begin{array}{l}\text { Median change in ACD } \\
\text { between } 12 \text { months } \\
\text { and baseline }\end{array}$ & $\begin{array}{l}218 \\
\text { (115 to } 448)\end{array}$ & $\begin{array}{l}470 \\
(210 \text { to } 1160)\end{array}$ & $\begin{array}{l}445 \\
\text { (165 to } 900)\end{array}$ & 003 \\
\hline
\end{tabular}

ACD denotes absolute claudication distance.

* Wilcoxon rank sum test.

Additional findings

No difference in the improvement in walking distance was found between patients using walking as the sole mode of exercise and patients performing a combination of exercises including cycling / spinning, rowing, stepping, and sports activities in general (Table 5).

Furthermore, there was no significant difference in improvement in walking distance between individual training and group training.
Table 5: Features of supenvised exercise programs, combining the SET without feedback and SET with feedback groups

\begin{tabular}{|c|c|c|c|}
\hline & $\begin{array}{l}\text { Walking exercise } \\
\text { only }\end{array}$ & $\begin{array}{l}\text { Combination of } \\
\text { exercises* }\end{array}$ & p-value** \\
\hline & $n=60$ & $\mathrm{n}=75$ & \\
\hline \multirow{5}{*}{$\begin{array}{l}\text { Median change in ACD between } \\
3 \text { months and baseline } \\
\text { Median change in ACD between } \\
12 \text { months and baseline }\end{array}$} & $280(128-492)$ & $252(150-460)$ & .692 \\
\hline & & & \\
\hline & $405(173-900)$ & $405(173-900)$ & .896 \\
\hline & Individual & Grouptraining & \\
\hline & $n=97$ & $n=33$ & \\
\hline $\begin{array}{l}\text { Median change in ACD between } \\
3 \text { months and baseline }\end{array}$ & $265(153-508)$ & $220(115-400)$ & .313 \\
\hline $\begin{array}{l}\text { Median change in ACD between } \\
12 \text { months and baseline }\end{array}$ & $410(170-1065)$ & $300(150-890)$ & .382 \\
\hline
\end{tabular}

* Consisting of cycling / spinning, rowing, stepping, and sports activities in general

** Wilcoxon rank sum test

\section{Discussion}

The results of this study suggest that the training volume offered in the first 3 months of supervised exercise therapy for intermittent claudication is related to the level of improvement in terms of walking distance at 3 and 12 months. Good results were observed with 2 or more training sessions per week each lasting more than 30 minutes. However, it must be noted that no significant further improvement was seen between 2 or more training sessions a week, nor between sessions lasting 30 to 45 minutes and those lasting over 45 minutes.

The results of our study are generally in line with those of two meta-analyses on optimal exercise SET training. 5,6 However, these meta-analyses found a frequency of 3 times a week 5 or 3 times a week or more ${ }^{6}$ to be optimal. Moreover, one study suggested that an optimized training program consists of a total training volume of 2000 minutes, ${ }^{5}$ whereas our results suggest a minimum training volume of 590 minutes within the first 3 months. Since the content of the therapy in our study was tailored to the patients' needs after 3 months, we could not evaluate the relation between total training volume and the effect on walking distance.

Results of studies using other exercise modalities than walking, for example polestriding ${ }^{7}$ and upper- and lower-limb ergometry, show improvements in walking ability in patients with PAD. These results suggest that any kind of training could improve walking distance for these patients. Our data show no difference between patients using walking as the sole mode of exercise and patients performing different exercises as well as walking. Researchers have suggested that resistance training does not augment the response to a walking exercise program. ${ }^{9}$ The added value of other type of exercises than walking in terms of improved walking distance is an interesting topic for further research, especially since it is imaginable that varying exercises can increase patients' compliance with exercise programs and facilitate the incorporation of exercise in their daily lives. 
The nature of the Dutch guidelines for physiotherapists does not allow us to determine an optimal duration of the program: the guideline recommends tailoring the program to the individual patient's needs, with training frequency for patients responding well to the exercise program being phased down sooner than that of patients showing minimal response. The fact that the therapists worked according to the guideline represented a limitation to our study. It was the physiotherapists who decided on the therapy frequency and duration for each patient, which may have led to selection bias. There is no consensus in the literature regarding the minimum duration of SET programs either, with recommendations varying from 10 to 14 weeks $^{10}$ and 12 to 24 weeks ${ }^{5}$ to more than 26 weeks. ${ }^{6} \mathrm{~A}$ randomized trial evaluating various durations and frequencies would be required to address this question satisfactorily and optimize the intervention.

Since the Dutch guidelines recommend walking up to sub-maximum pain, we were unable to compare between high and low intensity programs. Interestingly, one study reported in the literature suggested that the efficacy of low-intensity exercise (walking up to $40 \%$ of maximum workload) is similar to that of high-intensity exercise (walking up to $80 \%$ of maximum workload). 11 It has also been suggested that walking below the pain threshold level is effective in terms of increasing walking distances. ${ }^{12,13}$

Several studies have reported conflicting results regarding patient-related variables predicting walking distance after SET. ${ }^{14-17}$ A recent study identified baseline $A C D$, body mass index and current smoking as possible factors influencing treatment outcome. ${ }^{15}$ Cognitive factors, such as patients' belief in the effectiveness of SET, are also reported to be positively associated with the improvement in walking distance. ${ }^{14}$ Patients with a PAD in combination with a type $\mathrm{D}$ personality, defined as having a tendency to have negative emotions and being socially inhibited, remained more severely impaired after therapy (conservative and / or invasive) than other patients. ${ }^{18}$

In conclusion, our study suggests that a supervised exercise program consisting of 2 or more training sessions a week each lasting over 30 minutes during the first 12 weeks of a program that is later tailored to the patient's needs, with a maximum duration of 1 year, seems to optimize walking distance after 3 and 12 months.

\section{References}

1. Nicolai SP, Teijink JA, Willigendael EM, et al.

Multicenter randomized clinical trial of supenvised exercise therapy with or without feedback versus walking advice for intermittent claudication Submitted.

2. Bendermacher BL, Willigendael EM, Tiijnk IA Prins MH. Supervised exercise therapy versus non-supervised exercise therapy for intermittent claudication. Cochrane database of systematic reviews (Online). 2006(2):CD005263.

3. Jongert MWA, Hendriks HJM, Van Hoek J, et I. KNGF-richtlin Claudicatio Intermittens, Ned Tijdschr Fysiother 2003;Suppl:3-58.

4. Gardner AW, Skinner JS, Cantwell BW, Smith LK. Progressive vs single-stage treadmill tests for evaluation of claudication. Med Sci Sports Exerc. 1991;23:402-408.

5. Bulmer AC, Coombes JS. Optimising exercise training in peripheral arterial disease. Sports Med. 2004:34:983-1003.

6. Gardner AW, Poehlman ET. Exercise rehabilitation programs for the treatment of claudication pain A meta-analysis. JAMA. 1995;274:975-980.

7. Langbein WE, Collins EG, Orebaugh C, Maloney C, Williams KJ, Littooy FN, Edwards LC. Increasing exercise tolerance of persons limited by claudication pain using polestriding. J Vasc Surg 2002;35:887-893.

8. Walker RD, Nawaz S, Wilkinson CH, Saxton JM, Pockley AG, Wood RF. Influence of upper- and lower-limb exercise training on cardiovascular function and walking distances in patients with intermittent claudication. J Vasc Surg. 2000;31:662-669.

9. Hiatt WR, Wolfel EE, Meier RH, Regensteiner JG. Superiority of treadmill walking exercise versus strength training for patients with peripheral arterial disease. Implications for the mechanism of the training response. Circulation. 1994:90:1866-1874.
10. Martinez CA, Carmeli E, Barak S, Stopka CB. Changes in pain-free walking based on time in accommodating pain-free exercise therapy for peripheral arterial disease. J Vasc Nurs. 2009;27:2-7.

11. Gardner AW, Montgomery PS, Flinn WR, Katzel LI. The effect of exercise intensity on the response to exercise rehabilitation in patients with intermittent claudication. J Vasc Surg. 2005;42:702-709.

12. Mika P, Spodaryk K, Cencora A, Unnithan VB, Mika A. Experimental model of pain-free treadmill training in patients with claudication. Am JPhys Med Rehabil. 2005;84:756-762.

13. Mika P, Spodaryk K, Cencora A, Mika A. Re blood cell deformability in patients with claudication after pain-free treadmill training. Clin I Sport Med. 2006;16:335-340.

14. Rosfors S, Arnetz BB, Bygdeman S, Skoldo L, Lahnborg G, Eneroth P. Important predictors of the outcome of physical training in patients with intermittent claudication. Scandinavian journal of rehabilitation medicine. 1990;22:135-137.

15. Kruidenier LM, Nicolai SP, Ten Bosch JA, de Bie RA, Prins MH, Teijink JA. Predictors of Walking Distance After Supervised Exercise Therapy in Patients with Intermittent Claudication. Eur I Vasc Endovasc Surg. 2009;38:449-55

16. Andriessen MP, Barendsen GJ, Wouda AA, de Pater L. Changes of walking distance in patients with intermittent claudication during six months intensive physical training. VASA. 1989;18:63-68

17. Gardner AW, Katzel L, Sorkin JD, Killewich LA, Ryan A, Flinn WR, Goldberg AP. Improved functional outcomes following exercise rehabilitation in patients with intermittent claudication.J Gerontol A Biol Sci Med Sci. 2000;55:M570-577.

18. Aquarius AE, Denollet J, Vries JD, Hamming J. Poor health-related quality of life in patients with peripheral arterial disease: Type D personality and severity of peripheral arterial disease as independent predictors. J Vasc Surg. 2007:46:507-12 


\section{CHAPTER 11}

General discussion 


\section{General discussion}

Intermittent claudication is the commonest manifestation of peripheral arterial disease (PAD) and is experienced by patients as a moderate to severe impairment in walking ability. Even more importantly, intermittent claudication represents generalized atherosclerosis and is associated with an elevated risk of cardiovascular events and death. The studies described in this thesis focus on diagnosis, evaluation, and treatment of intermittent claudication.

\section{Ankle brachial index}

The ankle brachial index $(\mathrm{ABI})$ represents the ratio of the ankle to brachial systolic blood pressure. If atherosclerotic lesions cause narrowing of one or more lower extremity arteries, the ankle blood pressure decrease, resulting in a lower $\mathrm{ABI}$. $\mathrm{ABI}$ values lower than 0.9 are evidence of PAD. Moreover, an ABI $<0.9$ is associated with a substantially increased risk of cardiovascular events, even in patients not presenting with typical leg symptoms. ${ }^{1}$ The importance of the ABI for assessment of limb-related symptoms and as prediction of cardiovascular events stresses the need for correct determination of the $A B I$.

Data presented in chapter 2 of the thesis show that $A B I$ measurements performed with a pocket Doppler yield comparable results to measurements performed with vascular laboratory equipment. The difference in mean $\mathrm{ABI}$ of 0.05 in favor of vascular laboratory measurement may relate to the automated system, which automatically

visualizes Doppler signal output accompanied by an audible signal, resulting in a higher ankle blood pressure readings compared with a handheld Doppler device. It should be noted that both measurement techniques in this study were performed by experienced operators, which positively influences the ankle brachial measurement. ${ }^{2,3}$ Furthermore, the relatively high ankle pressures of the patients with intermittent claudication

included in this study are easier to measure than low ankle pressures in patients with severe PAD. Although, not relevant for the individual patient, the minor difference between both methods could change the prevalence of PAD in an epidemiological context. $^{4}$

The value and importance of the $A B I$ in primary care is increasingly being recognized. The study described in chapter 3 demonstrates that $A B I$ measurements performed in primary care with a pocket Doppler and $\mathrm{ABI}$ measurements in the vascular laboratory, albeit on average the same in the study population, greatly differed in individual patients. Remarkably, 30\% of patients referred from primary care with a low $A B I$ had a normal $A B I$ value in the vascular laboratory. Variability in techniques of blood pressure measurement and in calculation of the $A B I$ may account for the poor agreement in $\mathrm{ABI}$ measurements between primary care and vascular laboratory. Unfortunately, the study was underpowered for statistical comparison of the various approaches to $A B I$ measurement. Since the study was conducted in patients who were referred to the vascular surgeon by their general practitioner, the study population may not represent the total population of primary care patients presenting to the general practitioner with symptoms of $P A D$, and, hence, the sensitivity and/or specificity for the ABI in general practice cannot be calculated. 


\section{Evaluation of functional capacity in patients}

\section{with intermittent claudication}

Treadmill testing is the most frequently used assessment for the evaluation of disease severity and effect of treatment in patients with PAD. Parameters used to express the walking capacity are the distance at which claudication begins (initial claudication distance, ICD = pain free walking distance, PFWD) and the distance until exhaustion or cessation due to maximal leg pain (absolute claudication distance, $A C D=$ maxima walking distance, MWD). Unfortunately, the great variety of treadmill protocols used and recommended precludes comparative studies of the effectiveness of different treatment strategies to improve the functional capacity in patients with intermittent claudication..$^{5-7}$

In this thesis, a meta-regression analysis is presented to identify the most reliable protocol and the most reliable outcome measure. Studies presenting data regarding reliability of treadmill testing were collected. Inclusion criteria were a repeated continuous or graded test within three weeks. Furthermore, reliability had to be expressed as an intraclass correlation coefficient (ICC). Eight studies with a total number of 658 patients were included. Meta-regression on the ICC values indicated that the treadmill test is most reliable when the ACD is measured with a graded protocol. It should be noted that the ICC is one way to define reliability. Although nine studies were excluded from analysis because other measures of reliability were used, including coefficient of variation, mean difference, graphical representation, Pearson's correlation coefficient, or percentage relative precision, most of these studies were consistent with the results of the meta-analysis. Based on these findings, we suggest an adaptation of current guidelines with regard to treadmill testing.

The relevance of treadmill assessment to walking capacity in daily life may be argued 8,9 Treadmill walking requires less effort, since the moving tread and absence of wind resistance reduce patient workload. For walking outside, more balance and stability with small obstacles, starts, and stops are demanded. Furthermore, holding onto the handrails for assurance of stability hampers walking with a good walking posture. In addition, treadmill assessment is hampered by the purchase price of a treadmill, lack of adequately trained personnel to assist with the tests. Since treadmill testing is timeconsuming, it is not directly available to all physicians. Moreover, it is not possible to assess all patients with one single 'standard' protocol, which accordingly to chapter 4 should be a graded test with the ACD as the primary outcome measure.

Prior to treatment, approximately $15 \%$ of patients with PAD are not able to walk on a treadmill at $3.2 \mathrm{~km} /$ hour, starting with $0 \%$ incline, increasing $2 \%$ every 2 minutes ('standard' protocol), ${ }^{10}$ whereas walking distance after successful therapy can be assessed with the 'standard' protocol. Furthermore, in our experience (data not presented), 35\% of patients can walk for longer than 30 minutes on the 'standard' protocol after 6 months of supervised exercise therapy. Since the maximum duration of a physiotherapy session is 30 minutes, this is recorded for all patients as 1600 meters, resulting in a inability to adequately assess improvements or nuances in deterioration in walking distance for patients walking at least 30 minutes. Therefore, four new treadmill protocols, two with lower and two with higher workloads were compared with a 'standard' protocol (3.2 km/hour, 2\% increase every 2 minutes), as described in chapter 5 . In a pilot study, all four new protocols were evaluated for feasibility. Subsequently, the most feasible lower workload (2.0 km/hour and $2 \%$ increase every 4 minutes) and higher workload (4.4 km/hour and 2\% increase every minute) protocols were calibrated against the 'standard' protocol, yielding two simple equations to predict walking distance on the 'standard' protocol from the adapted protocols.

The quality of the two new protocols, evaluated by ICC and coefficient of variation, was similar to the quality of the 'standard' protocol. The quality of the equations used to predict walking distances on the 'standard' protocol as depicted in Bland Altman plots showed a moderate translation. Hence, this study enables estimation of walking distances on a 'standard protocol' derived from the actual walking distance measured on the high or low workload protocols. Since the adapted protocols were evaluated in two small patient cohorts ( $n=43$ and $n=23$ ), the results of this study should be interpreted with some caution. Although the ICC values adequately range between 0.89 and 0.95 , the coefficient of variation of the new protocols is higher than the coefficient of variation of 10 to $15 \%$ that is generally regarded as acceptable. In addition, for the lower workload protocol, extrapolation of the prediction equation to walking distances below 200 meters is not possible because it would lead to a longer walking distance on the 'standard' protocol. For the high workload it can be assumed that extrapolation yields adequate results. However, testing this would be challenging since other factors like cardiopulmonary condition may limit treadmill test results.

In 1990, Regensteiner et al. developed the walking impairment questionnaire (WIO) as an alternative to treadmill testing to assess functional walking ability in patients with PAD. " Since then the WIQ has frequently been used in studies evaluating the effect of therapy in patients with PAD. The WIQ contains three domains measuring three important factors of walking ability: walking distance, walking speed, and stair climbing. Until recently, the WIQ was only validated in English and Spanish. Even more important than language, walking distances were investigated with standard size American living blocks as a reference. Patients were asked to report the degree of difficulty walking one living block $(\approx 91$ meters) without stopping. In Europe, a standard size living block is not a general concept. Hence, in the European WIQ, patients are asked to report the degree of difficulty of walking a certain distance, e.g. 100 meters, without a specific reference standard. Furthermore, in the original WIQ, distances were expressed in feet (United States customary units) instead of meters.

In chapter 6, a Dutch version of the WIQ that was culturally adapted to the metric system is presented and validated. It is shown to be a reliable and valid questionnaire for the evaluation of patients with intermittent claudication. Overall, the Dutch questionnaire shows good reliability, expressed as internal consistency and test-retest reliability, for assessment of walking impairment in patients with PAD. Comparison of the WIQ with treadmill testing for assessment of the concurrent validity, showed a fairly strong correlation coefficient of 0.52 between the total WIQ score and the absolute claudication distance. The construct validity was expressed as the correlation coefficients between the domains of the RAND-36, the EuroQol, and the WIQ scores The most relevant domains of the RAND-36, physical functioning and pain, showed correlation coefficients of 0.70 and 0.35 , respectively. 
This study was conducted in 130 consecutive patients presenting at the outpatient clinic for vascular surgery with intermittent claudication. Inclusion criterion was a walking distance of less than 750 meters as assessed with a 'standard' treadmil test. The study population was a heterogeneous group of PAD patients; 36\% of these patients had at least one vascular intervention in their history. It is imaginable that a longer duration of the disease could lead to better estimation of the maximal achievable walking distance.

Furthermore, for the concurrent validity, which is the correlation between a new test and the gold standard measuring the same construct, results from the WIQ were compared with results from treadmill testing. The WIQ evaluates functional walking ability. However, it is questionable whether treadmill testing is indeed the gold standard since walking distance on a treadmill can not directly be translated to walking ability in daily life. ${ }^{8,9}$ Determining (construct) validity with a more disease specific questionnaire for PAD like the Kings college Vascular Quality of Life Questionnaire (VASCUQOL) or the claudication scale (CLAUS) would be an alternative.

The correlations found between the total WIQ score and the maximal walking distance as assessed by treadmill testing in our study are slightly lower than the correlations presented by Regensteiner et al. ${ }^{11}$ A possible explanation could be the 'standard' size living blocks which are applied as a reference standard in the American situation. It is known that people estimate distances inaccurately: estimates of distance can differ by up to 14.6 -fold from measured distances. ${ }^{12}$

We also show that the WIQ can be used as a tool to detect objective improvement in functional ability after supervised exercise therapy for patients with PAD. Ninety one consecutive patients were asked to fill out a self-administrable WIQ before and three months after starting supervised exercise therapy to improve their walking distance. Three statistical methods, including a Spearman correlation coefficient between the change in total WIQ score and the change in absolute claudication distance, linear regression analysis, and responder group analysis, all support the similar conclusion. Therefore, the results of this study justify the use of the total WIQ score as an alternative to treadmill testing in the evaluation of patients with PAD.

Similarly to data presented in chapter 6 , the correlation coefficient between the change in total WIQ score and the change in maximal walking distance is slightly lower than corresponding correlation coefficients (0.34 and 0.38) presented in literature. ${ }^{13,14}$ However, correlation coefficients in literature are calculated from the distance score instead of the total WIQ score. Furthermore, in literature, the American WIO, with standard size living blocks for reference, was used.

\section{Management of patients with intermittent claudication} Supervised exercise therapy

According to the TASC-II Inter-Society Consensus for the Management of Peripheral Arterial Disease and the American Heart Association practice guidelines for the management of patients with peripheral arterial disease, supervised exercise therapy (SET) is recommended as the initial approach for symptomatic relief in patients with PAD. ${ }^{15,} 16$ In the Atrium medical center, SET is provided in a community-based setting implying that patients are referred for supervised exercise therapy to adequately trained physiotherapists in the proximity of their homes.

In chapter 8, a cohort study is presented including all patients presenting with intermittent claudication at the outpatient clinic for vascular surgery in the Atrium medical center Parkstad between January 2005 and September 2006 who were referred for SET by the vascular surgeon. Patients with critical limb ischemia were not referred for SET. Results of this study show that SET is an effective approach to improve walking distance. The community-based approach is at least as effective as clinic-based SET with regard to improvement in walking distance. Furthermore, this study demonstrates that a satisfactory or dissatisfactory improvement in walking distance achieved after 3 months of therapy is likely to persist at 6 and 12 months of follow-up. The drop-out rate of about 50\% at 12 months is high; however, it is within the same range as that in previously reported trials of clinic-based SET for selective patient populations.

The multicenter randomized clinical trial presented in chapter 9 demonstrates that SET is more effective than walking advice alone in improving walking distance, WIQ scores, and quality of life in patients with intermittent claudication. In line with the meta-analysis by Bendermacher et al., ${ }^{17}$ SET results in improvement in walking distance of approximately 200 meters, as well as a clear benefit in the physical domain of the SF-36 and the WIQ scores compared with walking advice alone. These findings emphasize the clinical effectiveness of SET. Interestingly, Spronck et al. recently demonstrated that after 6 and 12 months patients with intermittent claudication benefited equally from either endovascular revascularization or supenvised exercise therapy. ${ }^{18}$ In neither study the effectiveness of SET was related to the level(s) of disease, i.e., is is not known whether SET is equally effective for aortoiliac disease and for femoropopliteal or crural lesions.

In our trial, additional daily feedback by a Personal Activity Monitor (PAM ® ), which continuously displayed a cumulative activity score, did not further improve the effectiveness of SET. Although approximately one third of patients reported having used the PAM only partially or not at all, the majority of patients ( $n=54$ ) used the PAM during the entire year. Remarkably, after 1 year, 31 patients preferred to continued to use the PAM, and 58 patients would advise other patients with PAD to use the PAM in addition to SET program (data not presented).

In terms of generalizability, patients willing to participating in this trial may represent those who have more interest in exercise. It is known, that participating in a physical activity trial affects patients' behavior. Hence, this potential bias may overestimate the improvements in walking distance after SET as well as after a sole walking advice in the study population as compared with the total population of patients with intermittent claudication referred for exercise therapy Based on the results of chapter 9, SET programs should be made available for 
all patients with intermittent claudication. A recent questionnaire in the United Kingdom shows that SET is an under-utilized treatment. ${ }^{19}$ Only $20 \%$ of the resident surgeons had access to SET. Lack of resources was addressed as the main reason why a supervised exercise set-up has not been achieved. Other possible barriers can be related to reimbursement policies of insurance companies and availability of adequately trained professionals who can provide supervised exercise therapy. Community-based supervised exercise programs provided by trained physiotherapists as developed in the Parkstad area are readily accessible to patients as well as vascular surgeons.

The data presented in Chapter 10 suggest that the optimal program during the first 3 months consists of $\geq 2$ training sessions per week with a duration $>30$ minutes to achieve better results for walking improvement. Generally, this is in line with two metaanalyses concerning this topic. ${ }^{20,21}$ Since the guideline 'Intermittent Claudication' of the Royal Dutch Society for Physical Therapy 22 recommends tailoring the exercise program towards the individual needs of the patients, we could only investigate the relation between content of program and walking distance in the first three months before tailorization. Interestingly, the effects measured at 3 months persisted after 12 months. This is in line with the results in chapter 8.

\section{Implications for practice and future research}

This thesis shows that $\mathrm{ABI}$ measurements by a pocket Doppler device are

interchangeable with vascular laboratory equipment $\mathrm{ABI}$ measurements provided that the measurement is performed by an experienced operator. Based on the findings of great variability in pocket Doppler ABI assessments between primary care and vascular laboratory, we recommend specialized training of primary health care workers in pocket Doppler $\mathrm{ABI}$ assessment. For successful implementation of $\mathrm{ABI}$ assessment for the diagnosis of PAD as well as for atherosclerosis screening, without the need for elaborate sophisticated vascular laboratory equipment.

The meta-regression analysis of treadmill protocols revealed that walking distance in patients with intermittent claudication is most reliably assessed using a graded protocol with the absolute claudication distance as the primary outcome measure. Preferably, guidelines on diagnosis of PAD should be adapted accordingly. Implementation of one uniform treadmill protocol allows comparison of treatment effects for patients with intermittent claudication. To extend the use of standardized treadmill testing to patients who are not able to walk on a 'standard' graded protocol and to patients who can walk without complaints for more than 30 minutes on a 'standard' protocol, two adapted lower and higher workload protocols were developed, respectively. Prediction equations were created for estimating walking distance on a 'standard' protocol from these adapted workload protocols:

Predicted walking distance standard protocol $=149.5+(0.38 *$ walking distance low protocol $)$

Predicted walking distance standard protocol $=695.1+(0.93 *$ walking distance high protocol $)$

The development of these protocols extends the range of treadmill testing from maximal 1600 meters $(\approx 30$ minutes on the 'standard' protocol) to 2740 meters $(\approx 30$ minutes on the high workload protocol translates into 2740 meters on the 'standard' protocol). For patients with a walking distance of less than 200 meters on the low protocol an adequate estimation of walking distance is not possible with this equation and further research on this part is required. These new protocols are currently used in routine clinical assessment of patients treated with SET or percutaneous vascula interventions for intermittent claudication.

The Dutch version of the WIQ, with cultural adaptation to the European metric system, is a reliable and valid alternative to treadmill testing to evaluate the effect of SET in patients with PAD. In line with this, the WIQ may also be a useful tool to assess the improvement in walking capacity after a percutaneous vascular intervention or vascular surgery. The use of the WIQ for quality of life assessment is a subject of future research, since our study demonstrated no significant correlation between the total WIQ score and generic quality of life questionnaires. Translation of this European WIQ allows broad implementation of this practical tool in other European countries.

Although a single walking advice is still common practice, ${ }^{19}$ our randomized clinical trial demonstrates that SET yields significantly more improvement in walking ability and quality of life than a single walking advice. SET provided by local physiotherapists in proximity to the patients' home is at least as effective as clinic-based SET. A SE program consisting of $\geq 2$ training sessions per week with a duration $>30$ minutes should be offered during the first 3 months. The effect of SET should be evaluated after 3 months, since satisfactory and dissatisfactory results are likely to persist thereafter. Hence, when walking distance fails to improve after 3 months of SET, invasive interventions should be considered. Further research is necessary to investigate the effectiveness of SET for different levels of atherosclerotic disease, e.g. aortoiliac versus femoropopliteal lesions. In this respect, the exercise versus endoluminal revascularisation (CLEVER) study for aortoiliac disease is currently recruiting patients. ${ }^{23}$ Furthermore, future studies will address the cost-effectiveness of SET versus walking advice.

Although exercise has shown efficacious to improve exercise performance in patients with $\mathrm{PAD}$, future research should be focused on the underlying pathophysiological mechanisms. Furthermore, the combination of patient related and therapy related factors influencing the improvement in walking distance should be topic of further research to optimize SET.

In conclusion, data presented in this thesis show that a specialized training for primary health care workers for the ABI measurement should be developed. Furthermore, it is demonstrated that the effect of therapy on walking ability of patients with intermittent claudication can be evaluated by treadmill testing or by questionnaire, the Dutch WIQ. Finally, a randomized clinical trial showed that supervised exercise therapy is more effective than a walking advice and should be made available for all patients with PAD. 


\section{References}

1. Fowkes FG, Murray GD, Butcher I, et al. Ankle

brachial index combined with Framingham Risk Score to predict cardiovascular events and mortality: a meta-analysis. JAMA. 2008;300:197-208,

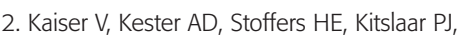
Knottnerus JA. The influence of experience on the reproducibility of the ankle-brachial systolic pressure ratio in peripheral arterial occlusive disease. Eur J Vasc Endovasc Surg. 1999;18:25-29.

3. Ray SA, Srodon PD, Taylor RS, Dormandy JA. Reliability of ankle:brachial pressure index measurement by junior doctors. Br J Surg. 1994:81:188-190.

4. Lange SF, Trampisch HJ, Pittrow D, et al. Profound influence of different methods for determination of the ankle brachial index on the prevalence estimate of peripheral arterial disease. BMC public health. 2007;7:147.

5. Heidrich H, Cachovan M, Creutzig A, Rieger H, Trampisch HJ. Guidelines for therapeutic studies in ontaine's stages II-IV peripheral arterial occlusive disease Cerman Society of Angiology. VASA 995;24:107-119.

6. Labs KH, Dormandy JA, Jaeger KA, Stuerzebecher CS. Hiatt WR. Transatlantic Conference on Clinical Trial Guidelines in Peripheral Arterial Disease clinical trial methodology. Basel PAD Clinical Trial Methodology Group. Circulation. 1999;100:e75-81.

7. ACSM's guidelines for exercise testing an prescription / American College of Sports Medicine. 7 ed. Baltimore: Lippincott Williams and Wilkins; 2006.

8. Siggaard-Andersen J, Petersen FB. Intermittent claudication. A comparison between subjective and measured claudication walking distance. Angiology. 1968;19:426-434.

9. Watson CJ, Phillips D, Hands L, Collin J. Claudication distance is poorly estimated and inappropriately measured. Br J Surg. 1997;84:1107-1109.
10. Kruidenier LM, Nicolai SP, Hendriks EJ, Bollen EC, Prins MH, Teijink JA. Supenvised exercise therapy for intermittent claudication in daily practice. J Vasc Surg. 2009;49:363-370.

11. Regensteiner JG, Steiner JF, Panzer RJ, Hiatt WR Evaluation of walking impairment by questionnaire in patients with peripheral arterial disease. J Vasc Med Biol. 1990;2:142-152.

12. Sharrack B, Hughes RA. Reliability of distance estimation by doctors and patients: cross sectional study. BMJ 1997:315:1652-1654.

13. Regensteiner JG, Steiner JF, Hiatt WR. Exercise training improves functional status in patients with peripheral arterial disease. J Vasc Surg 1996;23:104-115.

14. Regensteiner JG, Ware JE, Jr., McCarthy WJ, Zhang P, Forbes WP, Heckman J, Hiatt WR. Effect of cilostazol on treadmill walking, community-based walking ability, and health-related quality of life in patients with intermittent claudication due to peripheral arterial disease: meta-analysis of six andomized controlled trials. J Am Geriatr Soc 2002;50:1939-1946.

15. Norgren L, Hiatt WR, Dormandy JA, Nehler MR, Harris KA, Fowkes FG. Inter-Society Consensus for the Management of Peripheral Arterial Disease (TASC II).J Vasc Surg. 2007;45 Suppl S:S5-67.

16. Hirsch AT, Haskal Z, Hertzer NR, et al. ACC/AHA Guidelines for the management of patients with peripheral arterial diseases (lower extremity, renal, mesenteric and abdominal aortic): a collaborative report from the American Association for Vascular Surgery/Society for Vascular Surgery, Society for Cardiovascular Angiography and Interventions, Society for Interventional Radiology, Society for Vascular Medicine and Biology, and the American College of Cardiology/American Heart Association Task Force on Practice Guidelines (Writing Committee to develop guidelines for the management of patients with peripheral arterial disease). Circulation. 2005;21:e463-e654.
17. Bendermacher BL, Willigendael EM, Teijink JA, Prins MH. Supervised exercise therapy versus non-supervised exercise therapy for intermittent claudication. Cochrane database of systematic reviews (Online). 2006(2):CD005263.

18. Spronk S, Bosch JL, den Hoed PT, Veen HF Pattynama PM, Hunink MG. Intermittent claudication: clinical effectiveness of

endovascular revascularization versus supervise hospital-based exercise training-randomized controlled trial. Radiology. 2009;250:586-595.

19. Shalhoub J, Hamish M, Davies AH. Supervised exercise for intermittent claudication - an underutilised tool. Ann R Coll Surg Engl. 2009;91:473-6

20. Bulmer AC, Coombes JS. Optimising exercise training in peripheral arterial disease. Sports Med. 2004;34:983-1003.

21. Gardner AW, Poehlman ET. Exercise rehabilitation programs for the treatment of claudication pain. A meta-analysis. JAMA. 1995;274:975-980.

22. Jongert MWA, Hendriks HJM, Van Hoek J, et al. KNGF-richtlijn Claudicatio Intermittens. Ned Tijdschr Fysiother 2003;Suppl:3-58.

23. Murphy TP, Hirsch AT, Ricotta JJ, et al. The Claudication: Exercise Vs. Endoluminal Revascularization (CLEVER) study: rationale and methods. J Vasc Surg. 2008;47:1356-1363. 
Appendix 


\section{Walking Impairment Questionnaire}

Loopafstanden:

Zet een kringetje om de moeilijkheidsgraad die voor $U$ het beste omschrijft hoe het in de afgelopen week ging de beschreven afstanden te lopen zonder te stoppen.

\begin{tabular}{|c|c|c|c|c|c|}
\hline \multicolumn{6}{|c|}{ Moeilijkheidsgraad } \\
\hline Afstand & $\begin{array}{l}\text { Geen } \\
\text { probleem }\end{array}$ & $\begin{array}{l}\text { Klein } \\
\text { probleem }\end{array}$ & $\begin{array}{l}\text { Behoorlijk } \\
\text { probleem }\end{array}$ & $\begin{array}{l}\text { Ernstig } \\
\text { probleem }\end{array}$ & Onmogelijk \\
\hline $\begin{array}{l}\text { Binnenshuis } \\
\text { lopen }\end{array}$ & 4 & 3 & 2 & 1 & 0 \\
\hline 50 meter & 4 & 3 & 2 & 1 & 0 \\
\hline 100 meter & 4 & 3 & 2 & 1 & 0 \\
\hline 200 meter & 4 & 3 & 2 & 1 & 0 \\
\hline 275 meter & 4 & 3 & 2 & 1 & 0 \\
\hline 500 meter & 4 & 3 & 2 & 1 & 0 \\
\hline 1000 meter & 4 & 3 & 2 & 1 & 0 \\
\hline
\end{tabular}

Loopsnelheid:

Zet een kringetje om de moeilijkheidsgraad dat voor $U$ het beste omschrijft hoe het in de afgelopen week ging om de beschreven snelheid te lopen zonder te stoppen.

\begin{tabular}{|c|c|c|c|c|c|}
\hline \multicolumn{6}{|c|}{ Moeilijkheidsgraad } \\
\hline Afstand & $\begin{array}{l}\text { Geen } \\
\text { probleem }\end{array}$ & $\begin{array}{l}\text { Klein } \\
\text { probleem }\end{array}$ & $\begin{array}{l}\text { Behoorlijk } \\
\text { probleem }\end{array}$ & $\begin{array}{l}\text { Ernstig } \\
\text { probleem }\end{array}$ & Onmogelijk \\
\hline $\begin{array}{l}100 \text { meter } \\
\text { langzaam }\end{array}$ & 4 & 3 & 2 & 1 & 0 \\
\hline $\begin{array}{l}100 \text { meter } \\
\text { normaal }\end{array}$ & 4 & 3 & 2 & 1 & 0 \\
\hline $\begin{array}{l}100 \text { meter } \\
\text { snel }\end{array}$ & 4 & 3 & 2 & 1 & 0 \\
\hline $\begin{array}{l}100 \text { meter } \\
\text { rennend }\end{array}$ & 4 & 3 & 2 & 1 & 0 \\
\hline
\end{tabular}

Trap lopen:

Zet een kringetje om de moeilijkheidsgraad die voor $U$ het beste omschrijft hoe het in de afgelopen week ging het beschreven aantal trappen te lopen zonder te stoppen.

\begin{tabular}{lccccc}
\hline & Moeilijkheidsgraad & & & & \\
\hline $\begin{array}{l}\text { Trappen } \\
\text { Geen }\end{array}$ & $\begin{array}{c}\text { Klein } \\
\text { probleem }\end{array}$ & $\begin{array}{c}\text { Behoorlijk } \\
\text { probleem }\end{array}$ & $\begin{array}{c}\text { Ernstig } \\
\text { probleem }\end{array}$ & Onmogelijk \\
$\begin{array}{l}\text { Één trap } \\
\text { op lopen }\end{array}$ & 4 & 3 & 2 & 1 & 0 \\
$\begin{array}{l}\text { Twee } \\
\text { trappen op }\end{array}$ & 4 & 3 & 2 & 1 & 0 \\
$\begin{array}{l}\text { lopen } \\
\text { Drie } \\
\text { trappen op } \\
\text { lopen }\end{array}$ & 4 & 3 & 2 & 1 & 0 \\
\hline
\end{tabular}




\section{Likert scoring system Walking Impairment Questionnaire}

\begin{tabular}{llll}
\hline Domein afstand & Antwoord vragenlijst & Vermenigvuldigd met & \\
\hline Binnenshuis lopen & $\ldots$ & $* 7$ & $=\ldots$ \\
50 meter & $\ldots$ & $* 50$ & $=\ldots$ \\
100 meter & $\ldots$ & $* 100$ & $=\ldots$ \\
200 meter & $\ldots$ & $* 200$ & $=\ldots$ \\
275 meter & $\ldots$ & $* 275$ & $=\ldots$ \\
500 meter & $\ldots$ & $* 500$ & $=\ldots$ \\
100 meter & & $* 1000$ & $=\ldots$ \\
& & Tussenscore & $=\ldots$ \\
Score domein & & & $=$ Tussenscore / 8528 \\
afstand & & &
\end{tabular}

Domein snelheid Antwoord vragenlijst Vermenigvuldigd met

\begin{tabular}{llll}
100 meter langzaam & $\ldots$ & $* 2$ & $=\ldots$ \\
100 meter normaal & $\ldots$ & $* 4$ & $=\ldots$ \\
100 meter snel & $\ldots$ & $* 6$ & $=\ldots$ \\
100 meter rennend & $\ldots$ & $* 8$ & $=\ldots$ \\
\cline { 2 - 4 } & & & $=\ldots$ \\
$\begin{array}{l}\text { Tussenscore domein } \\
\text { snelheid }\end{array}$ & & $=\quad$ Tussenscore $/ 80$ \\
\hline
\end{tabular}

Domein trap lopen Antwoord vragenlijst Vermenigvuldigd met

\begin{tabular}{|c|c|c|c|}
\hline Domein trap lopen & Antwoord vragenlijst & Vermenigvuldigd met & \\
\hline Één trap op lopen & $\ldots$ & $* 13$ & $=\ldots$ \\
\hline Twee trappen op & & & \\
\hline lopen & $\ldots$ & $* 26$ & $=$. \\
\hline Drie trappen op & & & \\
\hline lopen & $\ldots$ & * 39 & $=$. \\
\hline & & Tussenscore & $=\ldots$ \\
\hline $\begin{array}{l}\text { Score domein trap } \\
\text { lopen }\end{array}$ & & & $=$ Tussenscore $/ 312$ \\
\hline
\end{tabular}

\begin{tabular}{llll}
\hline WiQ score totaal & & \\
& & & \\
\hline Domein afstand & & & \\
Domein snelheid & & & \\
Domein traplopen & & & \\
& & $=\ldots$ & \\
\cline { 2 - 5 } & Tussenscore & & Tussenscore $/ 3$ \\
WIQ score totaal & & &
\end{tabular}


Summary 


\section{Summary}

Peripheral arterial disease (PAD) is defined as stenosis in the lower extremity arteries due to atherosclerosis. The most common clinical presentation of PAD is intermittent claudication, described as pain in the leg muscles during exercise that rapidly subsides with rest. A large number of patients with PAD are asymptomatic.

Supervised exercise therapy (SET) is increasingly recognized as the foundation of symptomatic treatment of patients with PAD. It is important to realize that PAD is a manifestation of systemic atherosclerosis and that PAD is a strong marker for future cardiovascular events, including myocardial infarction and stroke. Hence, early identification of patients with symptomatic or asymptomatic PAD and secondary prevention of atherothrombotic events by cardiovascular risk factor management is crucial to improve life expectancy and quality of life in this large population. The aim of this thesis was to refine the diagnostic work-up and conservative treatment of patients with PAD.

The ankle brachial index $(A B I)$ is used for the diagnosis of PAD. A decreased $A B I$ as determined by the ratio between the systolic blood pressure at the ankle and at the arm identifies patients with PAD. In Chapter 2 the validity of ABI determination using a pocket Doppler device is compared with automatic vascular laboratory measurement in 99 patients suspected of PAD. A great correspondence with a small difference, albeit statistically significant, between the two methods was demonstrated. Hence, this chapter demonstrates that $\mathrm{ABI}$ measurements with an inexpensive pocket Doppler device and automatic vascular laboratory equipment yield comparable results and can replace each other.

Chapter $\mathbf{3}$ investigates the use of $\mathrm{ABI}$ measurements in primary care using pocket Doppler devices. In this cross-sectional study, consecutive patients suspected of PAD based on $\mathrm{ABI}$ assessment in primary care practices were included. The $\mathrm{ABI}$ measurements were repeated in the vascular laboratory. Furthermore, referring general practitioners were interviewed about method of measurement and calculation of the index. Great variability was demonstrated between the $A B I$ measured in primary care practice and in the vascular laboratory. Both the method of blood pressure measurement and the method of calculating the index differed greatly between primary care practices. Hence, a step-by-step guideline describing $\mathrm{ABI}$ measurement with a hand-held Doppler seems necessary.

Thus, although the ABI can reliably be determined with cheap and easy-to-use Doppler equipment, a clear guideline is required to allow broad implementation of pocket Doppler $\mathrm{ABI}$ assessment in primary and secondary health care to identify patients with atherosclerosis.

A wide range of treadmill protocols are used to assess disease severity and evaluate the effect of treatment in patients with PAD. The objective of Chapter $\mathbf{4}$ was to identify the most reliable treadmill protocol and outcome measurement (absolute claudication distance (ACD) initial claudication distance (ICD)) for the assessment of patients with PAD. Therefore, a meta-regression analysis, including trials determining the intraclass correlation coefficient (ICC) of continuous and/or graded treadmill protocols, was conducted. Eight studies, with a total of 658 patients, could be included. Our results demonstrated the highest reliability, expressed as an ICC of 0.95 (95\% Cl: 0.94 to 0.96), for treadmill testing when using a graded protoco 
together with the ACD as the primary outcome measure. This meta-regression analysis indicates that with regard to adequate comparison of future studies, guidelines for clinical trials on treatment of patients with peripheral arterial disease should preferably be adapted accordingly.

Although a standard graded treadmill protocol can be used to assess walking ability in the majority of patients with intermittent claudication, such a uniform protocol cannot be used in patients with either very limited maximal walking speed or extended walking distance. For these patients two adapted treadmill protocols were developed with either a lower or a higher workload as described in Chapter 5. First, four graded study treadmill protocols, two with lower and two with higher workloads than the 'standard' protocol, were developed and tested. To assess the low workload protocols, 43 patients with an $A C D<500 \mathrm{~m}$ were included. Twenty-three patients with an ACD between 1000 and $1600 \mathrm{~m}$ were included to assess the high workload protocols. The feasibility of the low workload protocol with $2.0 \mathrm{~km} / \mathrm{h}$ and a 2\% increase every 2 minutes and of the high workload protocol with $4.4 \mathrm{~km} / \mathrm{h}$ and a $2 \%$ increase every minute was highest. Therefore, they were calibrated against the 'standard' protocol and reliability was found to be comparable with the 'standard' protocol. Based on orthogonal regression analyses, equations to predict walking distances on the 'standard' protocol from the distances measured on the new protocols were calculated. The quality of the new protocols, as assessed by the ICC and coefficient of variation (COV), was similar to the quality of the 'standard' protocol. In conclusion, this study enables relevant estimations of walking distance as measured on a 'standard' treadmill protocol from a protocol with a lower workload, which might be necessary for patients with a very limited walking distance at the start of the therapy, with the following equation:

Predicted walking distance standard protocol $=149.5+(0.38 *$ walking distance low protocol $)$

For patients with an extended walking distance after therapy it is possible to measure this distance on a higher protocol and translate this to the 'standard' protocol with the following equation:

Predicted walking distance standard protocol $=695.1+(0.93 *$ walking distance high protocol $)$

Chapters $\mathbf{6}$ and $\mathbf{7}$ the Walking Impairment Questionnaire (WIQ) is evaluated as an alternative tool to treadmill testing to assess walking ability. The WIQ is a short and easy to fill out questionnaire which evaluates functional walking ability in three domains: walking distance, walking speed, and the ability to climb stairs. The WIQ was validated in the English and Spanish language in non-SI units (distances expressed as feet) and with American standard size living blocks as reference for certain walking distances. In Chapter $\mathbf{6}$ the Dutch translation of the WIQ, culturally adapted to the European (SI) metric system and without a reference standard, is presented. In this validation study, the validity and reliability of the translated and culturally adapted WIQ were determined. With an internal consistency determined by the Cronbach's alpha of 0.92, a test-retest reliability expressed by the ICC of 0.89 , and significant correlations between the WIQ, the ACD and two quality of life questionnaires, the Dutch version can be considered as an internally consistent, reliable, and valid questionnaire for the evaluation of walking impairment in patients with intermittent claudication. Even more importantly, this European version of the WIQ allows broad implementation of this practical tool with only a simple language translation for other European countries.
In Chapter $\mathbf{7}$ we investigated whether the WIQ can be used as an alternative tool to treadmill testing to detect objective improvement in functional ability in response to SET in patients with intermittent claudication. Ninety-one patients with intermittent claudication who were referred for SET were included in this study. The WIQ, quality of life questionnaires (RAND-36 and EuroQol), and treadmill testing were administered at the study onset and after three months of SET. Responsiveness of the WIQ was determined by mean changes and correlation coefficients between the WIQ the ACD, and quality of life questionnaires. Furthermore, patients were categorized into quartiles based on the increase in $A C D$, which were subsequently related to change in WIQ and quality of life. The statistical methods all support a similar conclusion showing that a qualitative improvement in walking ability as assessed by the questionnaire corresponds with a quantitative improvement in walking distance as assessed by treadmill testing. In conclusion, this study suggests that the WIQ can be used as an alternative to treadmill testing as a primary outcome measurement to assess the efficacy of SET in patients with intermittent claudication. In line with this, the WIQ is also expected to be a useful tool for assessment of the efficacy of other interventions for intermittent claudication, for example percutaneous vascular interventions or vascular surgery.

The first step in the symptomatic treatment of patients with intermittent claudication according to the TransAtlantic Inter-Society Consensus (TASC-II) and the American Heart Association practice guidelines is SET. Most reports on SET for patients with intermittent claudication are situated in an outpatient setting. Limitations of this approach in routine clinical practice are insufficient capacity of the outpatient clinic and time and costs for the patient to visit this clinic several times a week.

Chapter 8 describes the results of SET in a community-based approach, consisting of a selected group of local physiotherapists especially trained in applying exercise therapy. In this prospective cohort study, 349 consecutive patients with intermittent claudication referred for SET were included. Exclusion criteria for SET were pain at rest or tissue loss. A total of 272 patients with intermittent claudication began the SET programme, and at one year, 129 of them (47.4\%) were available for analysis of walking distance. In the interim, 143 patients discontinued the SET programme for the following reasons: satisfaction with the achieved walking distance $(n=19)$ unsatisfying results $(n=26)$; not motivated $(n=22)$; (non)vascular intercurrent disease $(n=48)$; and other reasons $(n=28)$. The ACD increased significantly from a median of $400 \mathrm{~m}$ at baseline to $1100 \mathrm{~m}$ after 12 months of follow-up, corresponding to a median increase of $107.8 \%$. Although community-based SET had a high dropout rate, the results for improvement in walking distance were in line with the results from clinic-based SET as described in literature. Furthermore, the results showed that good and unsatisfactory results after three months usually persist in the individual patient and are useful to guide the physician in continuing SET or planning a percutaneous vascular or surgical intervention.

In conclusion, SET provided by local physiotherapists seems as efficacious as SET in a clinic-based approach in improving walking distance in patients with intermittent claudication. 
In Chapter 9 the efficacy of SET for patients with intermittent claudication with or without feedback provided by regional physiotherapists is compared with a single walking advice in a multicentre randomized trial, the EXITPAD study. Furthermore, it was hypothesized that additional daily feedback using an accelerometer increases the effectiveness of SET. In 11 study centres, 102, 109, and 93 patients were included in the walking advice, SET, and SET with feedback groups, respectively. Of these, 83,

93, and 76, respectively, could be analysed. The median change in ACD between 12 months and baseline was $110 \mathrm{~m}$ (interquartile range (IQR) 0 to $300 \mathrm{~m}$ ) in the walking advice group, $310 \mathrm{~m}$ (IQR 145 to $995 \mathrm{~m}$ ) in the SET-group, and $360 \mathrm{~m}$ (IQR 173 to $697 \mathrm{~m}$ ) in the SET with feedback group ( $P<0.001$ SET versus walking advice). WIQ scores and relevant domains of the quality of life questionnaire, the SF-36, improved statistically significantly in the SET-groups. This randomized study demonstrates that SET is more effective than a walking advice in improving walking distance by approximately $200 \mathrm{~m}$, which reflects a clinically relevant improvement. WIQ scores and quality of life also improved more in the SET-group than in the walking advice group. Additional feedback with an accelerometer did not result in further improvement.

In an additional analysis presented in Chapter 10, we explored the relation between the offered exercise protocols and the impact of the intensity of the SET programmes (in terms of frequency, duration, and type of exercise) on improvements in walking distance (response). The SET programmes offered to the patients of the EXITPAD study randomized to receive SET with or without feedback in the first three months were evaluated. Local physiotherapists of the 169 included patients filled out therapy evaluation sheets stating the frequency, duration, and types of exercises. This study suggests that a SET programme consisting of at least two training sessions a week, each lasting over 30 minutes, should be offered during the first three months of the SET programme to optimize improvement in terms of maximum walking distance.

In conclusion, PAD is one of the major manifestations of atherosclerosis and an important predictor of cardiovascular morbidity and mortality. Hence, adequate detection of patients with atherosclerosis is crucial to initiate cardiovascular risk management. SET improves walking ability and quality of life and should be the initia treatment for the majority of patients with intermittent claudication. Optimization of this treatment modality requires further research, for example studies on the cost effectiveness of SET, the effectiveness of SET for different levels of atherosclerotic disease, and facilitators of and barriers to widespread implementation of SET. This thesis is a call to action for all vascular professionals involved in care for patients with 
Samenvatting 
Perifeer arterieel vaatlijden wordt gedefinieerd als de aanwezigheid van een of meerdere stenoses door atherosclerose in de arteriën van de benen. Een groot deel van de patiënten met perifeer arterieel vaatlijden is asymptomatisch. De meest voorkomende klinische presentatie is claudicatio intermittens, gedefinieerd als pijn in de benen tijdens inspanning, snel verdwijnend in rust.

Gesuperviseerde looptherapie wordt steeds vaker (maar nog onvoldoende) gezien als de eerste en belangrijkste conservatieve behandeling om de loopcapaciteit van symptomatische patiënten met perifeer arterieel vaatlijden te verbeteren. Hiernaast is cardiovasculair risico managent, de behandeling van de aanwezige risicofactoren voor atherosclerose zoals roken, hypertensie, hypercholesterolemie, diabetes mellitus, overgewicht, dieet, van belang.

De aanwezigheid van perifeer arterieel vaatlijden is immers een manifestatie van systemische atherosclerose en geassocieerd met toekomstige cardiovasculaire complicaties, zoals een hartinfarct en / of een beroerte. Een vroege identificatie van patiënten met zowel symptomatisch als asymptomatisch vaatlijden is essentieel om cardiovasculaire complicaties te voorkomen dan wel uit te stellen in de tijd. Het doel van dit proefschrift was om het diagnostische traject en de conservatieve behandeling in de vorm van gesuperviseerde looptherapie te evalueren.

De enkel arm index (EAI) wordt gebruikt voor het stellen van de diagnose perifeer arterieel vaatlijden. Een afgenomen enkel arm index, bepaalt door de verhouding tussen de systolische bloeddrukken gemeten ter hoogte van de enkels en de armen, identificeert patiënten met perifeer arterieel vaatlijden. In hoofdstuk $\mathbf{2}$ werd de EAl meting met een eenvoudige, goedkope 'hand' Doppler vergeleken met de geautomatiseerde, uitgebreide apparatuur zoals gebruikt in een vaatlab. Hoewel een klein maar significant verschil gevonden werd tussen de twee methodes, was de overeenstemming goed. De klinische relevantie van het gevonden verschil was daarmee verwaarloosbaar. Concluderend toont dit hoofdstuk aan dat een EAl meting betrouwbaar verricht kan worden met een 'hand' Doppler en dat de resultaten vergelijkbaar zijn met de metingen in het vaatlab.

Hoofdstuk 3 onderzoekt het verrichten van EAl metingen in de eerste lijn met een 'hand' Doppler. In een cross-sectioneel onderzoek werden opeenvolgende patiënten met een verlaagde EAI meting in de eerste lijn en doorverwezen naar de tweede lijn met de differentiaal diagnose perifeer arterieel vaatlijden geïncludeerd. De EAl meting werd vervolgens herhaald in het vaatlab. Bovendien werden alle doorverwijzende huisartsen gevraagd deel te nemen aan een telefonische enquête over de methode van de meting en het berekenen van de index. Deze studie liet een grote variatie zien tussen de metingen in de eerste en de tweede lijn. Bovendien varieerden zowe de bloeddrukbepalingen als de berekening van de index tussen de verschillende praktijken in de eerste lijn. Een stapsgewiize richtlijn voor het meten van de EAI voor eerste- en tweedelijns professionals om adequaat patiënten met atherosclerose (perifeer arterieel vaatlijden) op te kunnen sporen, is noodzakelijk.

Patiënten met claudicatio intermittens zijn vooral beperkt in hun loopafstand. De loopcapaciteit wordt gebruikelijk uitgedrukt als de maximale loopafstand gemeten op een loopband. Verschillende protocollen met variërende snelheid en helling van de loopband worden gebruikt om de ernst van het ziektebeeld en / of het effect van de 
behandeling te beoordelen. Continue protocollen zijn protocollen met een continue snelheid en hellingshoek van de loopband en progressieve protocollen hebben een continue snelheid met een toenemende hellingshoek gedurende de test. Gebruikte uitkomstmaten zijn de maximale loopafstand en de pijnvrije loopafstand (het moment waarop de pijn in de benen tijdens het lopen begint). Het doel van hoofdstuk $\mathbf{4}$ was om na te gaan welk type protocol en welke uitkomstmaat het meest betrouwbaar is om patiënten met perifeer arterieel vaatlijden te evalueren. Daarom was een metaregressie analyse verricht van studies die de intra class correlatie coëfficiënt (ICC) van continue en / of progressieve loopband protocollen hebben onderzocht. Acht studies met in totaal 658 patiënten konden hiervoor worden geïncludeerd. Onze resultaten laten zien dat een progressieve test met de maximale loopafstand als uitkomstmaat het meest betrouwbaar is met een ICC van 0,95 (95\% betrouwbaarheidsinterval 0,94 tot 0,96). Op basis van deze meta-regressie analyse en met het oog op het adequaat vergelijken van loopband resultaten van verschillende studies, adviseren wij om een uniform protocol op te nemen in de behandelings richtlijnen en zeker ook de richtlijnen voor klinische studies die de behandeling van patiënten met perifeer arterieel vaatlijden onderzoeken.

Hoewel een standaard progressieve loopband test bij de meeste patiënten met perifeer arterieel vaatlijden gebruikt kan worden voor evaluatie, is dit bij patiënten met een zeer beperkte of juist een zeer lange loopafstand niet mogelijk. Voor aanvang van de looptherapie is deze test voor een deel van de patiënten met een zeer beperkte loopafstand gewoonweg te lastig. Bij een ander deel van de patiënten verbetert het loopvermogen zoveel door bijvoorbeeld het volgen van looptherapie dat het met de standaard test langer dan 30 minuten duurt voordat pijnklachten optreden. Dit is praktisch niet haalbaar en een test waarbij de pijnklachten sneller optreden is wenselijk.

Voor deze twee groepen patiënten worden in hoofdstuk $\mathbf{5}$ aangepaste loopband protocollen gepresenteerd met een lagere of hogere belasting dan het standaard protocol. Initieel werden vier progressieve loopbandtesten ontwikkeld, twee met een lagere en twee met een hogere belasting. Om de nieuwe loopbandtesten met een lagere belasting te testen konden 43 patiënten geïncludeerd worden met maximale loopafstand <500 meter. Drieëntwintig patiënten met een loopafstand tussen de 1000 en 1600 meter konden geïncludeerd worden om de loopbandtesten met een hogere belasting te evalueren. De uitvoerbaarheid van de protocollen met een snelheid van $2 \mathrm{~km}$ per uur en 2\% toename in hellingshoek per 2 minuten (lagere belasting dan standaard protocol) en $4,4 \mathrm{~km}$ per uur en $2 \%$ toename in hellingshoek per minuut was beter vergeleken met de andere 2 protocollen. De betrouwbaarheid van de nieuw ontwikkelde protocollen was bij allen gelijk aan de betrouwbaarheid van het standaard protocol. Om die reden werd voor de twee best uitvoerbare protocollen met behulp van orthogonale regressie omrekenfactoren bepaald om de behaalde loopafstand met een nieuw protocol te vertalen naar een overeenkomstige afstand met het standaard protocol. De loopafstand gemeten met het protocol met een lagere belasting kan herleid worden tot de loopafstand die gelopen zou zijn met het standaard protocol met de volgende formule:

Geschatte loopafstand standaard protocol $=149,5+(0,38 *$ loopafstand protocol $)$
Voor patiënten met een langere loopafstand kan de volgende formule gebruikt worden om de loopafstand gemeten met het protocol met een hogere belasting te herleiden tot de loopafstand die gelopen zou zijn op het standaard protocol:

Geschatte loopafstand standaard protocol $=695,1+(0,93 *$ loopafstand protocol $)$

Met behulp van deze twee additionele loopbandprotocollen is het nu mogelijk om een wijdere range uitkomstmaten bij patienten, uitgedrukt in loopafstanden, onderling te vergelijken. Ook is het nu beter mogelijk om een individuele patient in de tijd, voor en na een interventie (gesuperviseerde looptherapie, percutane dan wel conventionele vasculaire interventie) te vervolgen.

In de hoofdstukken 6 en 7 wordt de Walking Impairment Questionnaire (WIQ) geëvalueerd als alternatief voor een loopbandtest om de loopcapaciteit te testen. De WIQ is een korte en gemakkelijk in te vullen vragenlijst die drie domeinen van functionele loopcapaciteit evalueert; loopafstand, loopsnelheid en traplopen. De WIQ is gevalideerd in het Engels en het Spaans in niet-SI-eenheden (afstanden zijn uitgedrukt in feet) met daarbij het Amerikaanse 'living block' als referentiekader voor de afstand van 300 feet (ongeveer 91,5 meter). In hoofdstuk 6 werd de WIQ in het Nederlands vertaald zonder een referentiekader en aangepast aan SI-eenheden. In deze validatiestudie werden de validiteit en betrouwbaarheid van de vertaalde en aangepaste WIQ bepaald. De Nederlandse vertaling kon, met een interne consistentie van 0,92 (bepaald met de Cronbach's alpha), een test-retest betrouwbaarheid van 0,89 (bepaald met een ICC) en significante correlaties tussen de WIQ, de loopafstanden en twee kwaliteit van leven vragenlijsten, beschouwd worden als een intern consistente, betrouwbare en valide vragenlijst voor het evalueren van de functionele loopcapaciteit van patiënten met claudicatio intermittens. Noemenswaardig is dat deze Europese versie van de WIQ met een simpele vertaling in alle landen met SI-eenheden gemakkelijk geïncorporeerd kan worden.

In hoofdstuk 7 zijn we nagegaan of de WIQ gebruikt kan worden als alternatief voor een loopbandtest om een objectieve verbetering na gesuperviseerde looptherapie bij patiënten met claudicatio intermittens te meten. Eenennegentig patiënten met claudicatio intermittens die verwezen waren voor gesuperviseerde looptherapie, konden geïncludeerd worden in deze studie. De WIQ, twee kwaliteit van leven vragenlijsten (RAND-36 en EuroQol) en een loopbandtest werden uitgevoerd aan het begin van de studie en drie maanden na aanvang van de gesuperviseerde looptherapie. Aan de hand van gemiddelde veranderingen in en correlatie coëfficiënten tussen de WIQ, de loopafstanden en de kwaliteit van leven vragenlijsten, werd de betrouwbaarheid van de WIQ in het weergeven van objectieve veranderingen onderzocht. Bovendien werden patiënten ingedeeld in kwartielen gebaseerd op hun toename in loopafstand, welke vervolgens gerelateerd werden aan de gemeten veranderingen op de WIQ en de kwaliteit van leven vragenlijsten. Alle gebruikte statistische methodes ondersteunden de conclusie dat de kwalitatieve verbetering in loopcapaciteit gemeten met de WIQ overeenkomt met de kwantitatieve veranderingen in loopafstand gemeten met een loopbandtest. Deze studie suggereert 
dus dat de WIQ als alternatief voor een loopbandtest gebruikt kan worden om de effectiviteit van gesuperviseerde looptherapie bij patiënten met claudicatio intermittens te evalueren. Het is aannemelijk dat de WIQ eveneens een bruikbaar instrument is om de effectiviteit van een percutane dan wel conventionele vasculaire interventie te evalueren.

Volgens de vigerende richtlijnen, de TransAtlantic Inter-Society Consensus (TASCII) en de American Heart Association practice guidelines, is de eerste stap in de symptomatische behandeling van patiënten met claudicatio intermittens; gesuperviseerde looptherapie. De meeste gepubliceerde studies die gesuperviseerde looptherapie voor claudicatio intermittens onderzoeken, werden verricht in een klinische setting. Nadelen hiervan zijn de beperkte capaciteit van de afdelingen revalidatie en / of fysiotherapie en de tijd en kosten die gemoeid zijn met het transport van patiënten naar het ziekenhuis of de kliniek, zoals bekend meerdere malen per week.

Om deze reden is het concept van gesuperviseerde looptherapie in de woonomgeving van de patiënt ontwikkeld waarvan in hoofdstuk $\mathbf{8}$ de resultaten worden beschreven. Gesuperviseerde looptherapie wordt gegeven door een selecte groep lokale fysiotherapeuten die getraind zijn in het geven van looptherapie. In deze prospectieve cohort studie konden 349 opeenvolgende patiënten met claudicatio intermittens, verwezen voor gesuperviseerde looptherapie, geïncludeerd worden. Exclusie criteria waren rustpijn en / of weefselverlies. In totaal 272 patiënten begonnen daadwerkelijk met het gesuperviseerde looptherapie traject en na 1 jaar kon van 129 $(47,4 \%)$ patiënten de loopafstanden geëvalueerd worden. Ondertussen stopten 143 patiënten met het looptherapie programma met de volgende redenen; tevreden met het behaalde resultaat $(n=19)$, onvoldoende resultaat $(n=26)$, onvoldoende motivatie ( $n=22)$, overige ziekten $(n=48)$ en andere redenen $(n=28)$. De mediane maximale loopafstand, die 400 meter was bij aanvang van de studie, nam significant toe tot 1100 meter na 1 jaar. Dit komt overeen met 107,8\% toename. Hoewe gesuperviseerde looptherapie in de woonomgeving van de patiënt een hoge uitval kent, zijn de resultaten voor de loopafstand overeenkomstig met de resultaten beschreven in de literatuur voor klinische looptherapie programma's. Bovendien lieten onze resultaten zien dat een goed of een slecht resultaat na 3 maanden meestal blijvend was en een goede leidraad is voor de behandelend arts om wel of niet door te gaan met het looptherapie programma. Concluderend is gesuperviseerde looptherapie voor patiënten met claudicatio intermittens door fysiotherapeuten in de woonomgeving van patiënt even goed als looptherapie aangeboden in de vorm van een klinisch programma.

In hoofdstuk 9 wordt een gerandomiseerde multicentrische studie, de EXITPAD studie, die de effectiviteit van gesuperviseerde looptherapie in de woonomgeving van de patiënt met of zonder feedback voor patiënten met claudicatio intermittens vergelijkt met een eenmalig mondeling loopadvies, gepresenteerd. Verondersteld werd dat het gebruik van een bewegingsmeter, die dagelijks feedback geeft op het bewegingspatroon, in combinatie met gesuperviseerde looptherapie effectiever zou zijn, resulterend in een langere loopafstand. In de 11 deelnemende studie centra konden 102, 109 en 93 patiënten geïncludeerd worden in respectievelijk de loopadvies groep, de gesuperviseerde looptherapie groep en de gesuperviseerde looptherapie met feedback groep. In deze groepen konden na 12 maanden 83, 93 en 76 patiënten geanalyseerd worden. De mediane verandering in maximale loopafstand tussen 12 maanden en baseline was 110 meter (interkwartielafstand (IKA) 0 tot 300 meter) in de loopadvies groep, 310 meter (IKA 145 tot 995 meter) in de gesuperviseerde looptherapie groep en 360 meter (IKA 173 tot 697 meter) in de gesuperviseerde looptherapie met feedback groep $(P<0,001$ loopadvies groep vergeleken met beide gesuperviseerde looptherapie groepen). De WIQ scores en relevante domeinen van de kwaliteit van leven vragenlijst, de SF-36, verbeterden significant in de gesuperviseerde looptherapie groepen. Deze studie toont aan dat gesuperviseerde looptherapie effectiever is dan een eenmalig loopadvies met een klinisch relevant verschil in verbetering van ongeveer 200 meter. Ook de WIQ scores en kwaliteit van leven scores verbeterden meer in de gesuperviseerde looptherapie groepen dan in de loopadvies groep. Het geven van feedback met behulp van een bewegingsmeter bleek niet van toegevoegde waarde.

In een additionele analyse, gepresenteerd in hoofdstuk $\mathbf{1 0}$, is de relatie tussen de verschillende aangeboden oefenprotocollen, de impact van de intensiteit van de gesuperviseerde looptherapie programma's (met aandacht voor frequentie, duur en soort oefeningen) en de verbeteringen in loopafstand bekeken (dosis-respons). De looptherapie programma's aangeboden aan de patiënten van de EXITPAD studie, die gerandomiseerd waren in de gesuperviseerde looptherapie groepen met of zonder feedback, in de eerste 3 maanden van de therapie werden geëvalueerd. De regionale fysiotherapeuten van de 169 geïncludeerde patiënten werden gevraagd therapie evaluatie formulieren in te vullen over de frequentie, duur en inhoud van het oefenprogramma. Deze analyse suggereert dat een oefenprogramma van ten minste twee therapie sessies per week met een minimale duur van 30 minuten aangeboden zou moeten worden in de eerste 3 maanden van het oefenprogramma om de toename in loopafstand te optimaliseren.

Concluderend is perifeer arterieel vaatlijden een belangrijke manifestatie van atherosclerose en een belangrijke voorspeller van cardiovasculaire morbiditeit en mortaliteit. Een adequate detectie van patiënten met atherosclerose is van groot belang om cardiovasculair risicomanagement te initiëren. Gesuperviseerde looptherapie verbetert de loopcapaciteit en kwaliteit van leven en zou de initiële symptomatische behandeling moeten zijn voor alle patiënten met claudicatio intermittens. Het optimaliseren van deze behandeling vereist meer onderzoek, bijvoorbeeld naar de kosteneffectiviteit van gesuperviseerde looptherapie, de effectiviteit van gesuperviseerde looptherapie bij verschillende aangedane trajecten van de bloedvaten en naar facilitatoren en barrières voor het wijdverbreid implementeren van gesuperviseerde looptherapie. 


\section{Dankwoord}


Graag wil ik een aantal mensen bedanken die mij geholpen hebben bij het tot stand brengen van dit proefschrift.

Om te beginnen met mijn copromotor Joep Teijink. Joep, zonder jouw wetenschappelijke inzicht, creativiteit, onuitputtelijke energie en enthousiasme was er geen promotieplek en dus ook geen proefschrift geweest. Al voor dat ik begonnen was met mijn onderzoek, kreeg ik een uitnodiging voor de winterbijscholing. Een mooier begin is niet voorstelbaar. Joep, bedankt!

Mijn promotor Martin Prins. Beste Martin, ondanks dat dankwoorden volgens jou onnodig zijn, wil ik je bedanken voor je grote betrokkenheid. De rust die jij had om telkens weer nauwgezet te luisteren naar de onderzoeksvorderingen en mij met raad en daad bij te staan, zijn essentieel geweest voor het tot stand komen van dit proefschrift. Na elk bezoek zat ik weer vol energie.

Ellen Rouwet, samen zijn we een tijdje 'de dikke en de dunne' geweest. Giechelend, maar met jouw uiterste nauwkeurigheid hebben we ons samen door menige tekst heen gewerkt. Ellen, je bent een geweldige copromotor.

Lotte Kruidenier, dokter (en sinds 2 maanden ook doctor) Lottie, mijn 'roomie'. Samen twee jaar in een kamer van nog geen 9 vierkante meter. We hebben elkaar intensiever gezien dan onze eigen familie. Je bent van onvoorstelbare waarde geweest voor zowel de onderzoeksvorderingen als een heleboel gezelligheid. Ik hoop in de toekomst nog veel met je samen te werken.

De EXITPAD studiegroep, met name Rob de Bie, Erik Hendriks en Marie-Louise Bartelink. Jullie hebben mij vele malen voorzien van tips en trics om de studie soepeler te laten verlopen. Veel dank gaat ook uit naar mijn voorgangsters Edith Willigendael en Bianca Bendermacher, die veel voorbereidend werk hebben verricht om de EXITPAD onderzoekslijn op te zetten.

Zonder centra in een multicenter onderzoek geen patiënten, en zonder patiënten geen onderzoek. Dank aan de vaatchirurgen en studiecoördinatoren van alle participerende ziekenhuizen. Zonder jullie was het nooit gelukt om deze studie tot een goed einde te brengen. Veel dank ben ik verschuldigd aan alle deelnemende patiënten en alle fysiotherapeuten, zowel van het Netwerk Looptherapie Parkstad (NLTP) als alle fysiotherapeuten verspreid in het hele land die door mij met mailtjes belaagd werden.

Wolfgang Viechtbauer en Pieter Leffers, veel dank voor de deskundige hulp bij het uitvoeren van menig analyse.

Math Smeets, dank voor ons onderdak, 'het AOA-aquarium', op jouw Acute Opname Afdeling. Veel succes met je eigen promotieonderzoek.

Mijn twee kamermaatjes van de laatste maanden. Jan Ten Bosch (Janus het was gezellig samen, bij deze een lebber van mij) en Dennis Japink, allebei bedankt voor de gezelligheid en veel succes met het afronden van jullie promotieonderzoeken. 
Maartje van den Broek, collega arts-onderzoekster uit het MUMC Maastricht en vriendin; ik hoop op nog veel van die gezellige (stap)avonden. Ook jij veel succes met je onderzoek.

Een van de vele leuke aspecten van het arts-onderzoekerschap, was het samenwerken met studenten geneeskunde (WESPen en keuze-co's),

bewegingswetenschappen en fysiotherapie, die via Joep de afgelopen jaren

passeerden. Marieke, Marie-Chantal, Kim, Katja, Kirsten, Michel, Jeroen, Marjolein Ivette, Ralf, Roel, Miriam, Mieke, Christiane, Ricky, Brenda, Verona, Veerle en Eefke, bedankt voor al jullie bijdrages. Onderdelen van jullie acties zijn terug te vinden in dit proefschrift.

Ik wil de maatschap chirurgie en de assistenten chirurgie van het Atrium medisch centrum hartelijk danken voor het opnemen van mij als arts-onderzoeker binnen de 'club'.

De nurse practitioners, altijd goed voor een hoop gezelligheid. Janine, Anouk en Ed, wat vond ik het leuk om jullie te leren kennen. Hartelijk dank voor het steeds weer opspeuren van alle mogelijke studiepatiënten. Ook Simone, Ria, Ron, de dames van het secretariaat en alle andere medewerk(st)ers van de afdeling chirurgie, bedankt voor alle hulp en de nodige afleiding tussen de onderzoekstaken door.

De dames van het vaatlab in Heerlen wil ik graag bedanken voor het meten van alle enkel arm indices, de basis van twee publicaties in dit boekje. Hans Kragten cardioloog, wil ik van harte bedanken voor de samenwerking in het 'project $X$ '. Kim Smolderen en Patrick Vriens bedankt voor de prettige samenwerking met de afdeling psychologie/vaatchirurgie uit Tilburg.

De beoordelingscommissie, Prof. dr. Jacobs, Prof. dr. ir. Van den Brandt, Prof. dr. Büller, Dr. Hoofwijk (bij wie ik in het Orbis Medisch Centrum mijn eerste schreden als arts heb mogen zetten) en Prof. dr. Metsemakers, wil ik hartelijk danken voor het beoordelen van het manuscript en de bereidheid om deel te nemen aan deze mooie dag.

lk prijs mij gelukkig met al mijn lieve vrienden en vriendinnen. Op het moment dat ik het onderzoek even beu was, kon ik altijd bij jullie terecht. Dank jullie wel voor alle gezelligheid, etentjes, feestjes en (jaarclub)uitjes die we samen hebben gedaan en nog gaan doen!

In het bijzonder Iris, Ik heb je leren kennen tijdens het eerste college van onze studie geneeskunde en het klikte meteen. Ik ben ontzettend blij dat jij een van mijn paranimfen wil zijn.

Mijn lieve schoonfamilie, Sjaak, Bep en Marieken, altijd weer geïnteresseerd in 'dat rare medische vak.' Bedankt voor jullie interesse en continue ondersteuning.

Mijn familie, Pap, Mam, Pol en Wendy, jullie hebben altijd achter mij gestaan en vertrouwen in mij gehad. Bedankt, zonder jullie was ik nooit gekomen waar ik nu ben. 
Curriculum Vitae 
Saskia Patricia Anna Nicolai was born on 17 March 1979 in Maastricht, The Netherlands. After graduating from secondary school (Sint Maartenscollege) in Maastricht, she started medical school at Maastricht University in 1997. During medical school, in 2001, she moved to Barcelona, Spain, to follow an internship in the department of Vascular Surgery of the University Hospital Bellvitge (Head Dr. M.A. Cairols). She obtained her medical degree in 2004

Thereafter, she worked as a resident at the department of Surgery at the Orbis Medical Center in Sittard (Head Dr. A.G.M. Hoofwijk). In 2006, based on a ZonMW grant applied for by the EXITPAD study group, she became a PhD student at the department of Vascular Surgery of the Atrium Medical Center, Parkstad Heerlen (Dr. J.A.W. Teijink) in close collaboration with Maastricht University (Prof. dr. M.H. Prins). The EXITPAD study, in combination with several initiated research projects, led to this thesis. In January 2010 she started her training programme in General Surgery at the Máxima Medical Center in Eindhoven/Veldhoven (Head Dr. W.J. Prakken).

Saskia Nicolaï and Michiel Pronk are the parents of Thom (28 March 2009). 

